\title{
Electronic Structure and Bonding in Iron(II) and Iron(I) Complexes Bearing Bisphosphine Ligands of Relevance to Iron-Catalyzed C-C Cross-Coupling
}

Jared L. Kneebone, Valerie E. Fleischauer, Stephanie L. Daifuku, Ari A. Shaps, Joseph M. Bailey, Theresa E. Iannuzzi and Michael L. Neidig*

Department of Chemistry, University of Rochester, Rochester, New York 14627, USA

E-mail: neidig@chem.rochester.edu 


\section{Table of Contents}

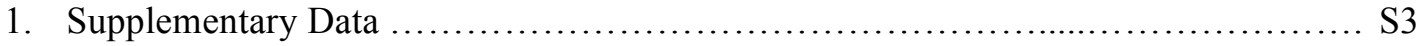

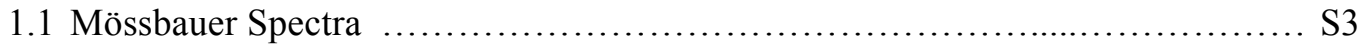

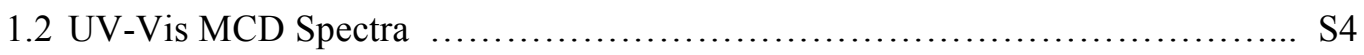

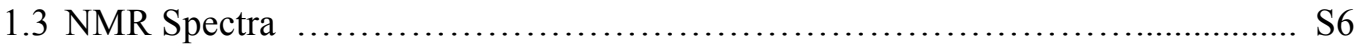

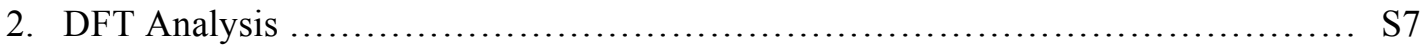

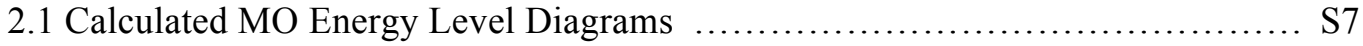

2.2 TD-DFT Assignments of CT Transitions for $\mathrm{FeCl}_{2}$ (bisphosphine) Complexes .... S12

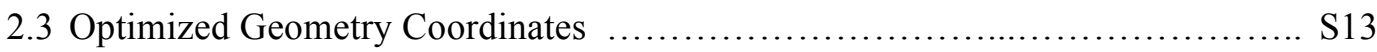

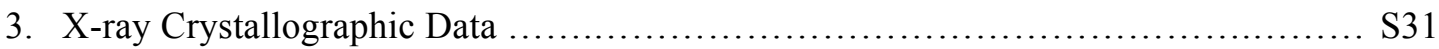

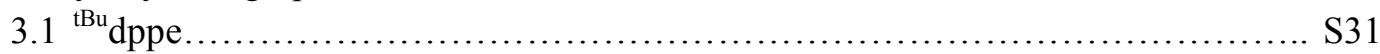

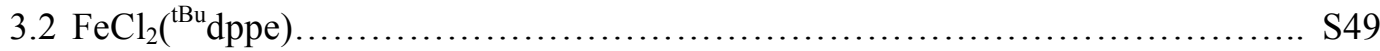

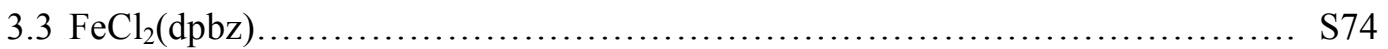

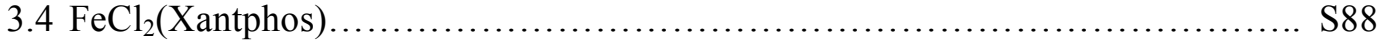

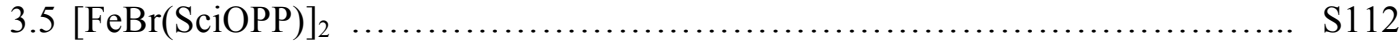




\section{Supplementary Data}

\subsection{Mössbauer Spectra}

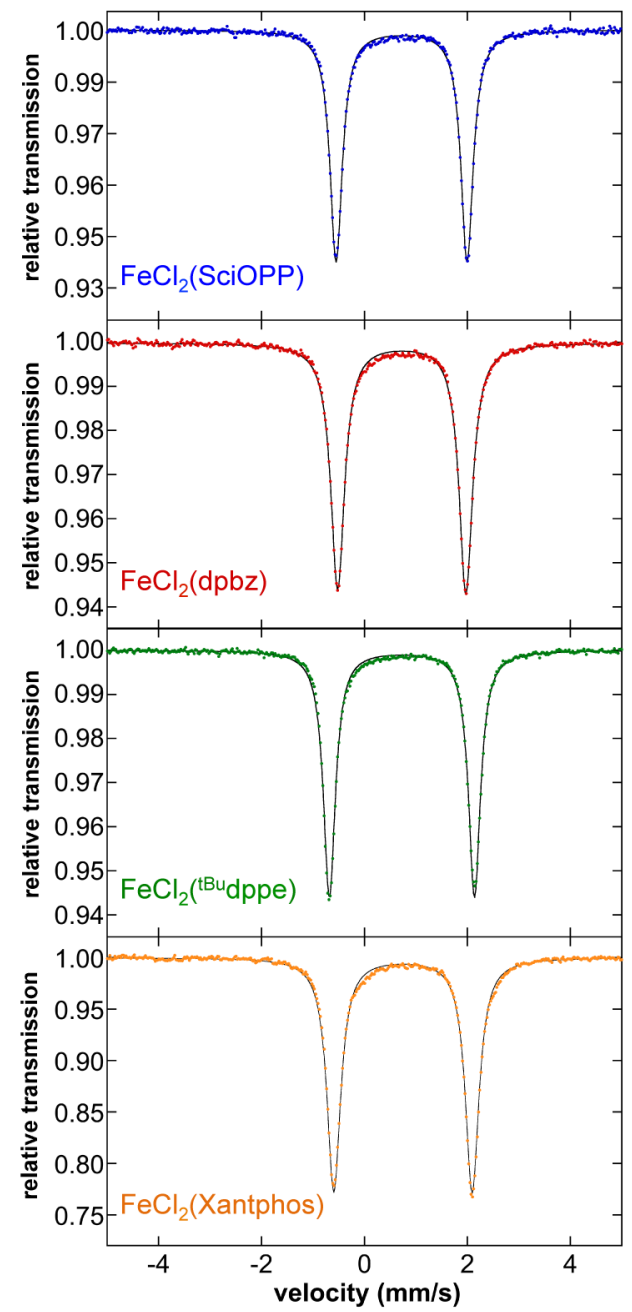

Figure S1. $80 \mathrm{~K}$, Solid-state ${ }^{57} \mathrm{Fe}$ Mössbauer spectra of $\mathrm{FeCl}_{2}$ (bisphosphine) complexes. The best-fit parameters for each complex are given in the manuscript in Table 1. 


\subsection{UV-Vis MCD Spectra}

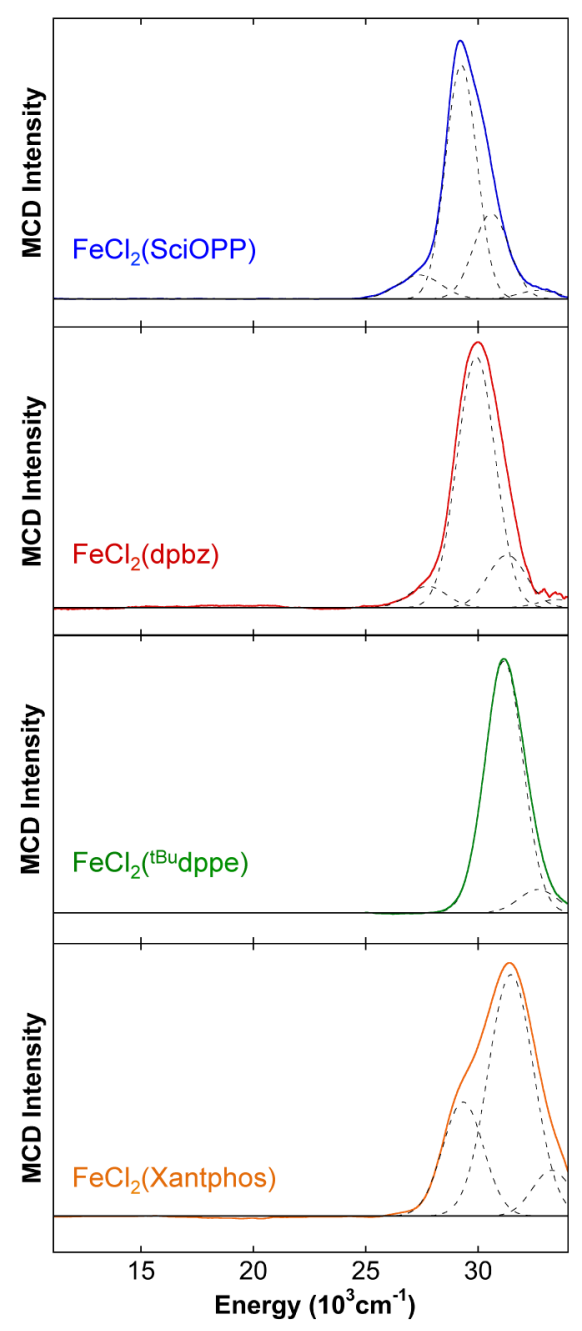

Figure S2. UV-Vis MCD spectra of $\mathrm{FeCl}_{2}$ (bisphosphine) complexes. Data (solid lines) and individual transition Gaussian fits (dashed lines) are given for each complex. The $\mathrm{FeCl}_{2}(\mathrm{SciOPP})$ spectrum was collected at $15 \mathrm{~K}$ and $7 \mathrm{~T}$ whereas all other spectra were collected at $5 \mathrm{~K}$ and $7 \mathrm{~T}$. All spectra were collected in $6: 1$, toluene- $\mathrm{d}_{8}$ : benzene- $\mathrm{d}_{6}$ except for $\mathrm{FeCl}_{2}$ (Xantphos) which was collected on a solid mull sample. 


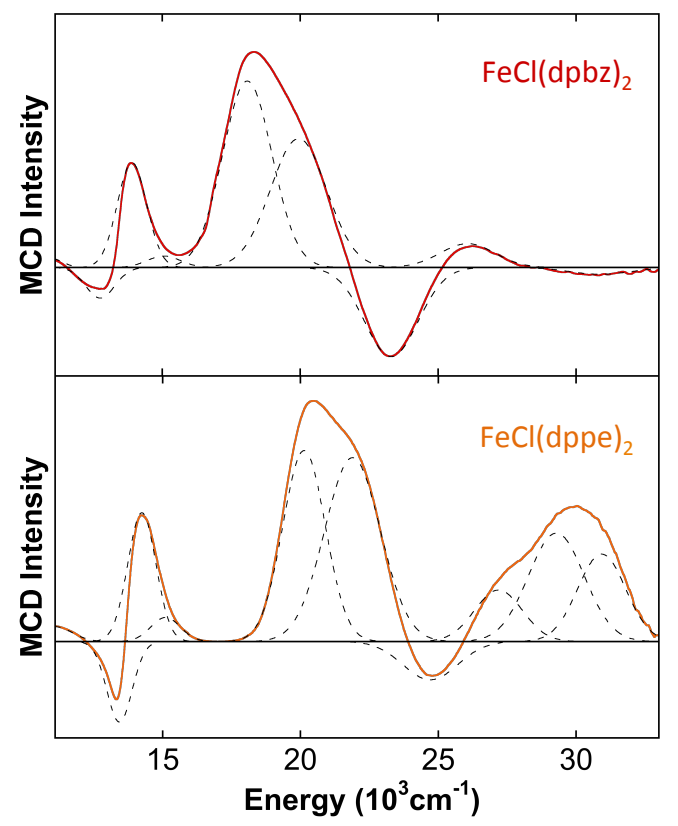

Figure S3. $5 \mathrm{~K}, 7 \mathrm{~T} \mathrm{UV}-\mathrm{V}$ is $\mathrm{MCD}$ spectra of $\mathrm{FeCl}(\mathrm{dpbz})_{2}$ and $\mathrm{FeCl}(\mathrm{dppe})_{2}$. Data (solid lines) and individual transition Gaussian fits (dashed lines) are given for each complex. Spectra were collected in 6:1, toluene- $\mathrm{d}_{8}:$ benzene- $\mathrm{d}_{6}$ 


\subsection{NMR Spectra}
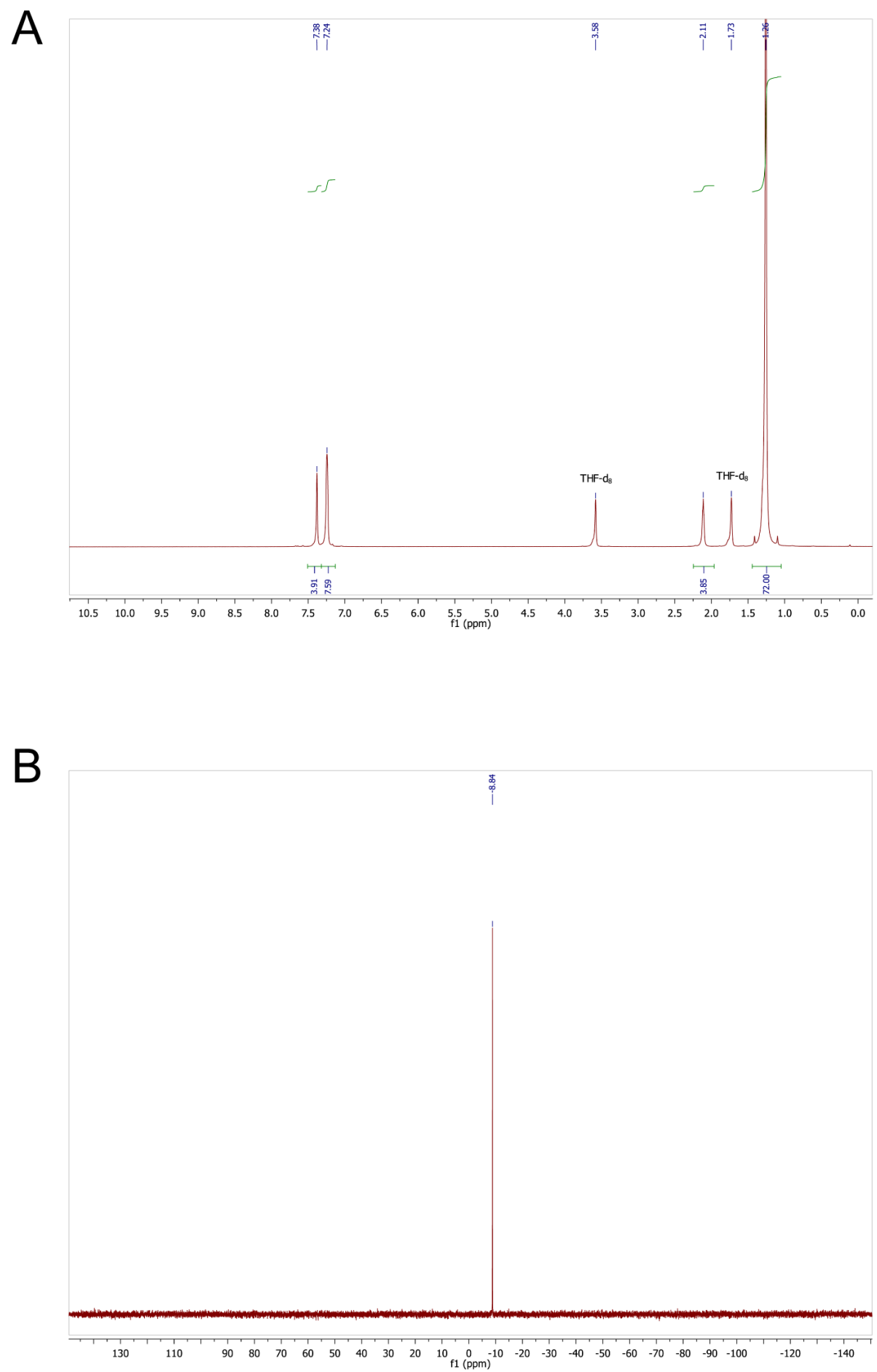

Figure S4. (A) ${ }^{1} \mathrm{H}$ NMR (400 MHz) and (B) ${ }^{31} \mathrm{P}$ NMR (400 MHz) of ${ }^{\mathrm{tBu}} \mathrm{dppe}$. 


\section{DFT Analysis}

\subsection{Calculated MO Energy Level Diagrams}
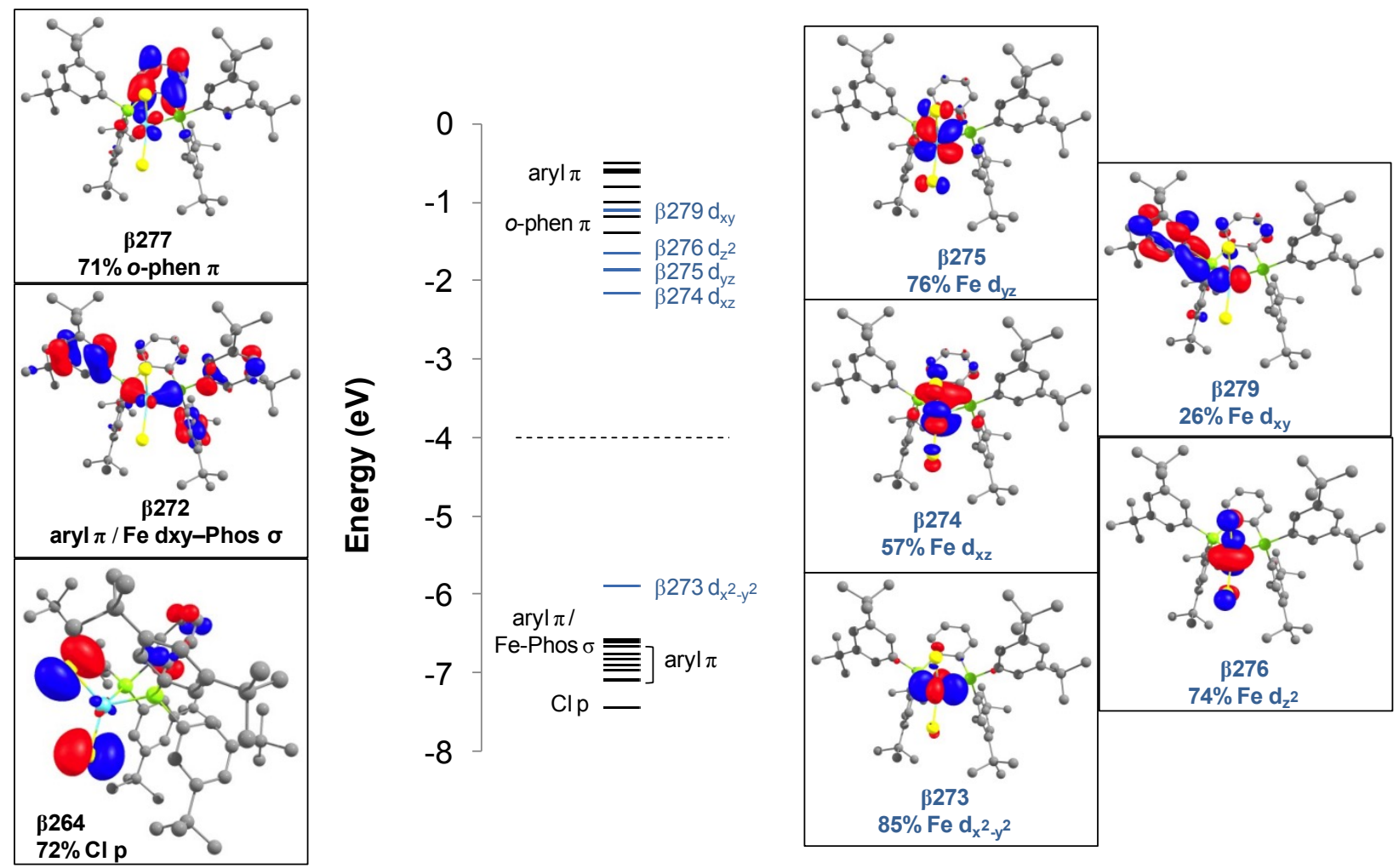

Figure S5. Calculated FMO energy level diagram for $\mathrm{FeCl}_{2}(\mathrm{SciOPP})$ and selected orbital depictions. 

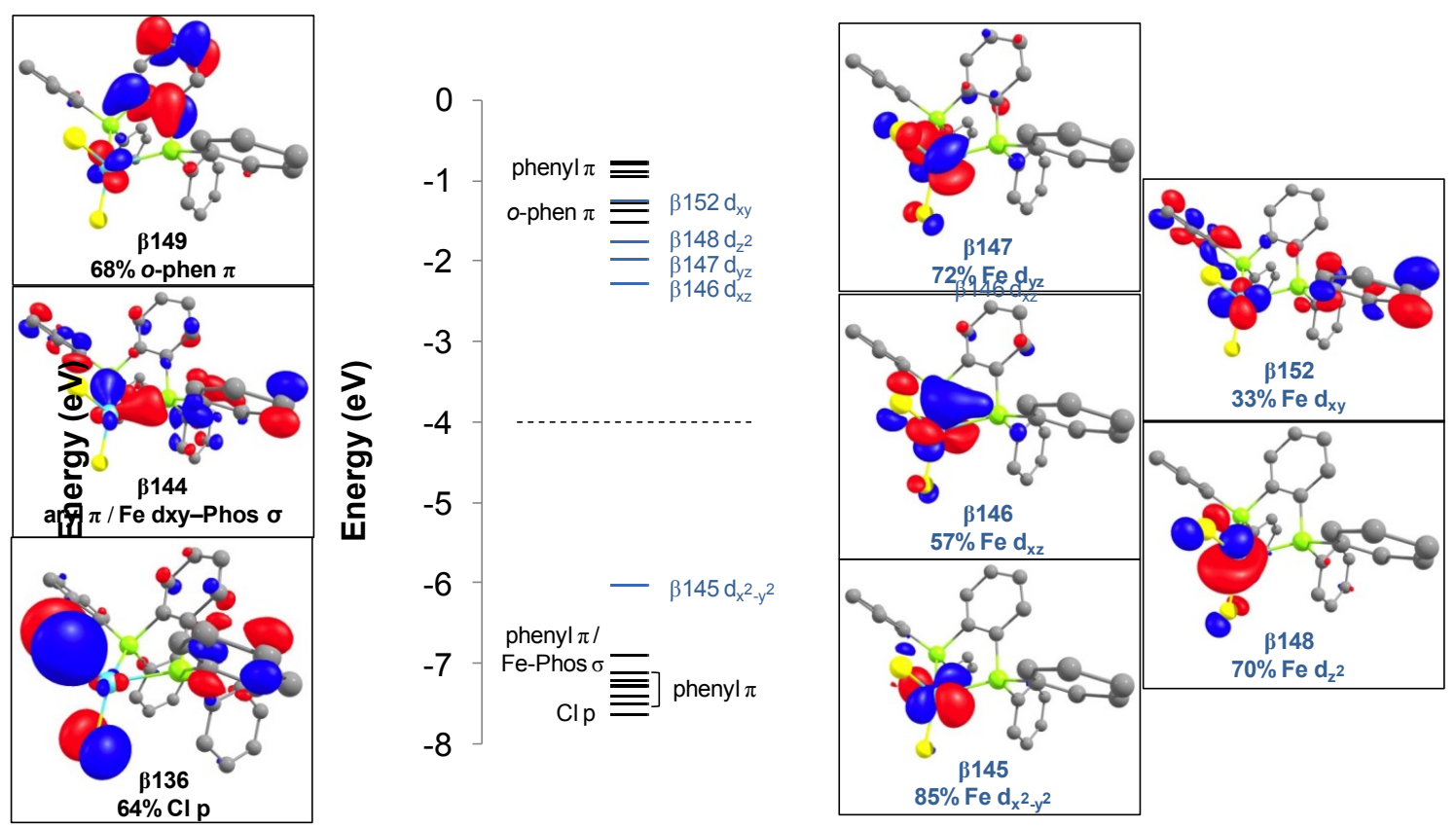

Figure S6. Calculated $\mathrm{MO}$ energy level diagram for $\mathrm{FeCl}_{2}(\mathrm{dpbz})$ and selected orbital depictions.
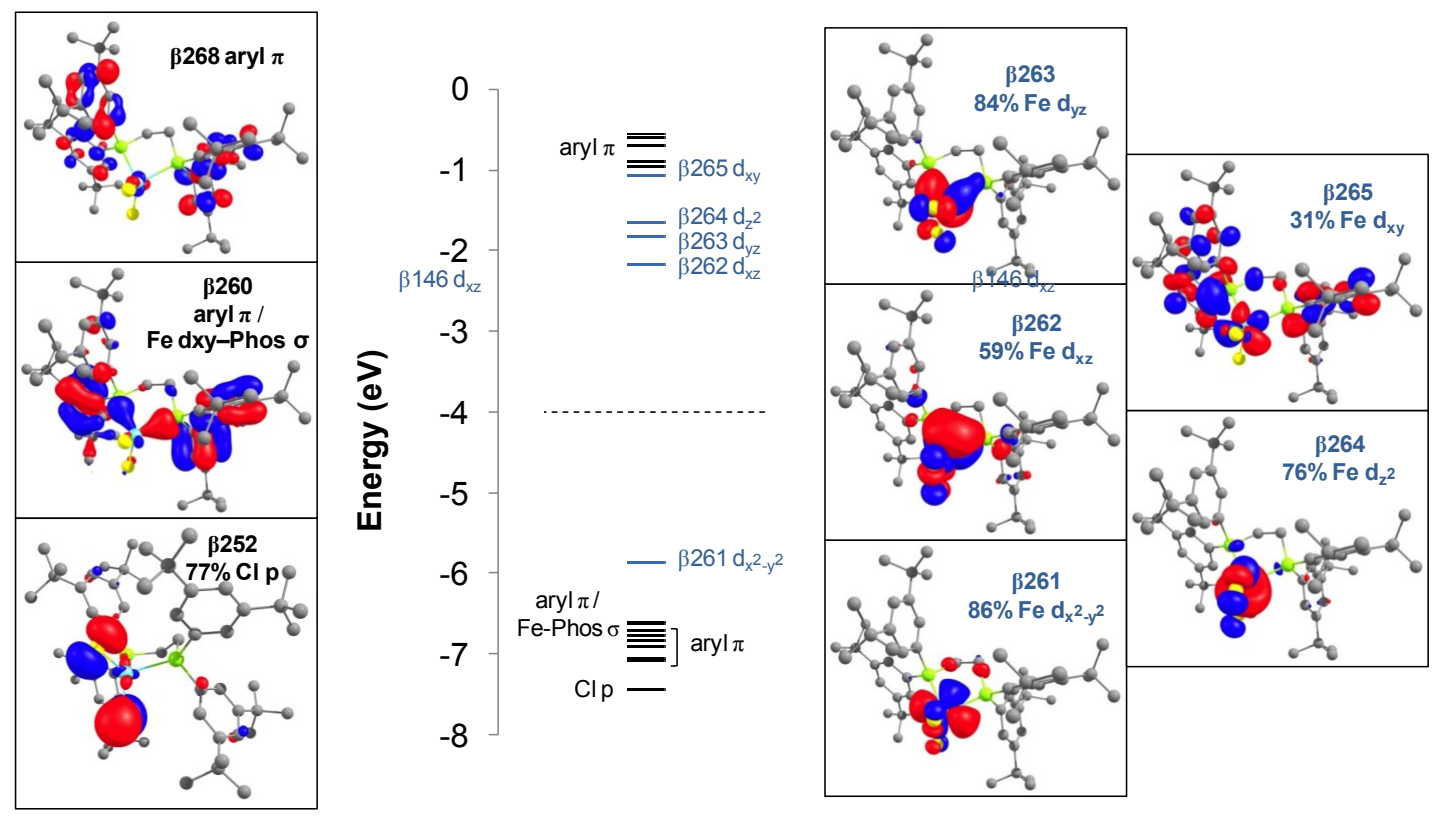

Figure S7. Calculated FMO energy level diagram for $\mathrm{FeCl}_{2}\left({ }^{\mathrm{CBu}} \mathrm{dppe}\right)$ and selected orbital depictions. 

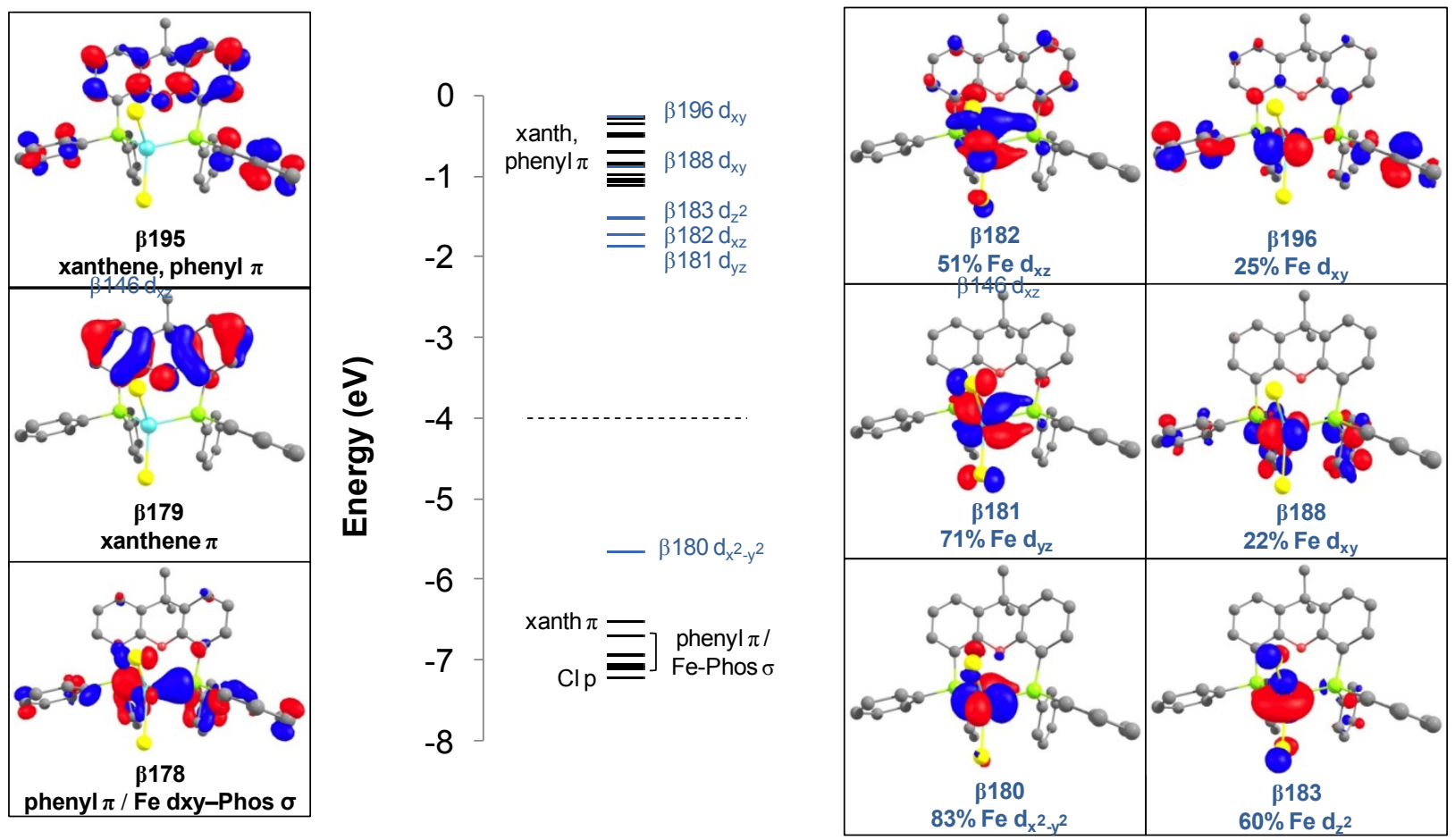

Figure S8. Calculated FMO energy level diagram for $\mathrm{FeCl}_{2}$ (Xantphos) and selected orbital depictions. 

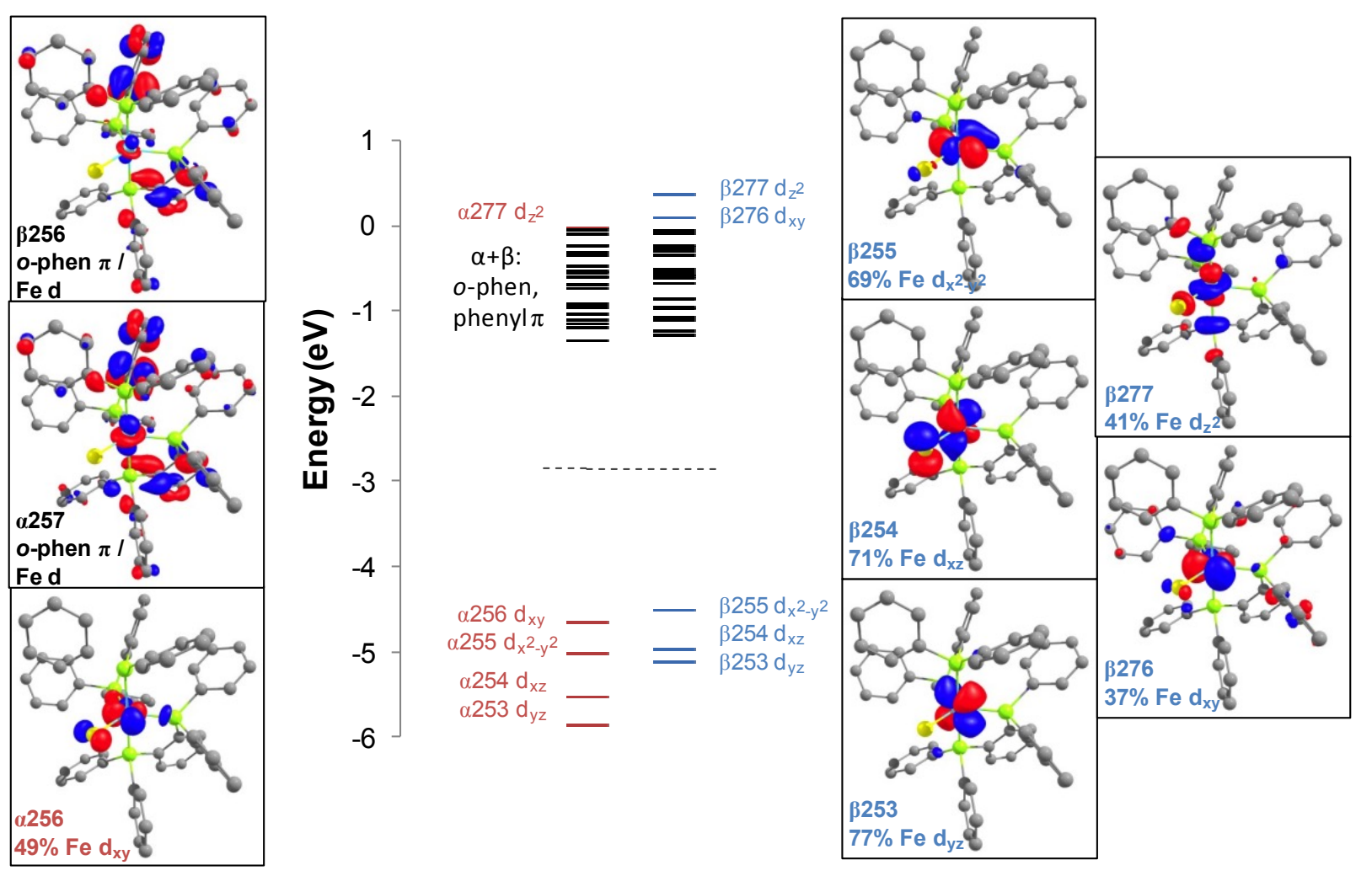

Figure S9. Calculated FMO energy level diagram for $\mathrm{FeCl}(\mathrm{dpbz})_{2}$ and selected orbital depictions. 


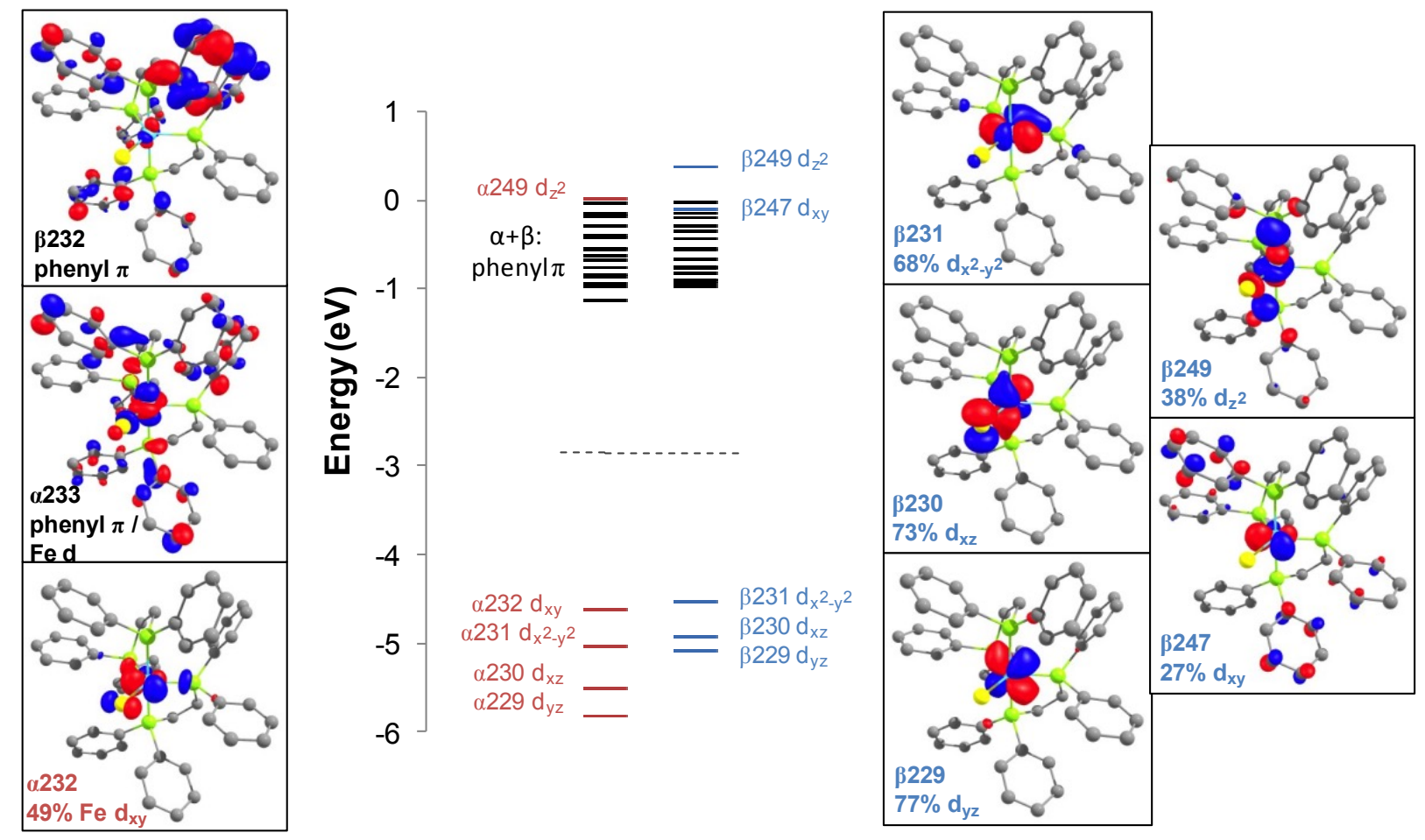

Figure S10. Calculated FMO energy level diagram for $\mathrm{FeCl}(\mathrm{dppe})_{2}$ and selected orbital depictions. 


\subsection{TD-DFT Assignments of CT Transitions for $\mathrm{FeCl}_{2}$ (bisphosphine) Complexes}

$\mathrm{FeCl}_{2}$ (SciOPP). Observed transitions in the UV-Vis MCD spectrum of $\mathrm{FeCl}_{2}(\mathrm{SciOPP})$ have been assigned using TD-DFT calculations. The high energy CT transitions observed by UV-Vis MCD are quite mixed, with dominant transition character in the bands at $27390 \mathrm{~cm}^{-1}$ and $29250 \mathrm{~cm}^{-1}$ deriving from MLCT within the $\alpha$ manifold from Fe $\mathrm{d}_{\mathrm{xy}}$ to low lying acceptor MOs of $o$-phen $\pi$ character. The band at $30590 \mathrm{~cm}^{-1}$ is composed of mixed $\alpha$ (MLCT) and $\beta$ (LMCT) character, with Fe $\mathrm{d}_{\mathrm{xy}} \rightarrow o$-phen $\pi$ character in the $\alpha$ manifold and aryl $\pi / \mathrm{Fe} \mathrm{d}_{\mathrm{xy}}$-Phos $\sigma \rightarrow \mathrm{Fe} \mathrm{d}_{\mathrm{xz}} / \mathrm{d}_{\mathrm{yz}}$ in the $\beta$ manifold. The last observed CT transition at $32685 \mathrm{~cm}^{-1}$ is again mixed but the dominant transition character is LMCT from aryl $\pi$ based MOs to $F e \mathrm{~d}_{\mathrm{xz} / \mathrm{yz}}$.

$\mathbf{F e C l}_{2}(\mathbf{d p b z})$. Observed transitions in the UV-Vis MCD spectrum of $\mathrm{FeCl}_{2}(\mathrm{dpbz})$ have been assigned using TD-DFT calculations. Assignments of the high energy CT transitions of $\mathrm{FeCl}_{2}(\mathrm{dpbz})$ are similar to those of $\mathrm{FeCl}_{2}$ (SciOPP) reflecting the consistent $o$-phen backbone structure in both complexes. The transitions observed at $27750 \mathrm{~cm}^{-1}$ and $29920 \mathrm{~cm}^{-1}$ are dominated by transitions in the $\alpha$ manifold bearing $\mathrm{Fe} \mathrm{d}_{\mathrm{xy}} \rightarrow o$-phen $\pi$ transition character. The band at $31290 \mathrm{~cm}^{-1}$ is composed of mixed $\alpha$ (MLCT) and $\beta$ (LMCT) character, with Fe $\mathrm{d}_{\mathrm{xy}} \rightarrow o$-phen $\pi$ character in the $\alpha$ manifold and phenyl $\pi / \mathrm{Fe} \mathrm{d}_{\mathrm{xy}}$-Phos $\sigma \rightarrow \mathrm{Fe}$ $\mathrm{d}_{\mathrm{xz}} / \mathrm{d}_{\mathrm{yz}}$ character in the $\beta$ manifold. The highest energy transition at $33530 \mathrm{~cm}^{-1}$ is dominantly composed of $\beta$ LMCT character in the form of phenyl $\pi / \mathrm{Fe} \mathrm{d}_{\mathrm{xy}}-\mathrm{Phos} \sigma \rightarrow \mathrm{Fe} \mathrm{d}_{\mathrm{xz}} / \mathrm{d}_{\mathrm{yz}}$.

$\mathrm{FeCl}_{2}\left({ }^{\mathrm{tBu}} \mathrm{dppe}\right)$. Observed transitions in the UV-Vis MCD spectrum of $\mathrm{FeCl}_{2}\left({ }^{\mathrm{tBu}} \mathrm{dppe}\right)$ have been assigned using TD-DFT calculations. Unlike both $\mathrm{FeCl}_{2}(\mathrm{SciOPP})$ and $\mathrm{FeCl}_{2}(\mathrm{dpbz})$, the $\mathrm{CT}$ region for $\left.\mathrm{FeCl}_{2}{ }^{\mathrm{t}}{ }^{\mathrm{Bu}} \mathrm{dppe}\right)$ is void of transitions below $30000 \mathrm{~cm}^{-1}$, reflecting the lack of backbone $\pi$-based MOs in the case of $\mathrm{FeCl}_{2}\left({ }^{\mathrm{tBu}} \mathrm{dppe}\right)$ and the fact that LLCT transitions bearing minimal Fe d character are predicted by TD-DFT to be dominant in this region. The two experimental CT transitions at $31150 \mathrm{~cm}^{-1}$ and 32670 $\mathrm{cm}^{-1}$ are both dominated by $\beta$ LMCT (aryl $\pi / \mathrm{Fe} \mathrm{d}_{\mathrm{xy}}-\mathrm{Phos} \sigma \rightarrow \mathrm{Fe} \mathrm{d}_{\mathrm{xz}} / \mathrm{d}_{\mathrm{yz}}$ ) transition character.

$\mathrm{FeCl}_{2}$ (Xantphos). Observed transitions in the UV-Vis MCD spectrum of $\mathrm{FeCl}_{2}$ (Xantphos) have been assigned using TD-DFT calculations. The high energy CT transitions observed by UV-Vis MCD are quite mixed, with dominant transition character in the band at $29330 \mathrm{~cm}^{-1}$ deriving from LMCT in the beta manifold with phenyl $\pi / \mathrm{Fe} \mathrm{d}_{\mathrm{xy}}$-Phos $\sigma \rightarrow \mathrm{Fe} \mathrm{d}_{\mathrm{xz}}$ transition character. The two higher energy transitions at $31460 \mathrm{~cm}^{-1}$ and $33280 \mathrm{~cm}^{-1}$ derive intensity from $\alpha$ MLCT to low lying ligand-based acceptor orbitals ( $\mathrm{Fe} \mathrm{d}_{\mathrm{x} 2-\mathrm{y} 2} \rightarrow$ xanth, phenyl $\pi$ ). 


\subsection{Optimized Geometry Coordinates}

\subsection{1 $\mathrm{FeCl}_{2}(\mathrm{SciOPP})$ optimized with uPBEPBE/TZVP (toluene PCM)}

$\begin{array}{llll}26 & 4.154518000 & 18.770086000 & 5.727238000 \\ 17 & 2.057797000 & 18.878341000 & 4.924956000 \\ 17 & 5.622381000 & 20.412095000 & 5.391102000 \\ 15 & 3.986997000 & 17.906150000 & 7.978061000 \\ 15 & 4.966765000 & 16.552091000 & 5.251707000 \\ 6 & 3.689280000 & 16.086356000 & 7.728498000 \\ 6 & 3.001567000 & 15.308363000 & 8.672655000 \\ 6 & 2.795523000 & 13.944309000 & 8.456212000 \\ 6 & 3.265745000 & 13.344607000 & 7.284009000 \\ 6 & 3.933564000 & 14.112248000 & 6.327703000 \\ 6 & 4.156914000 & 15.481746000 & 6.538877000 \\ 6 & 2.599075000 & 18.424342000 & 9.090831000 \\ 6 & 2.811733000 & 18.847189000 & 10.412751000 \\ 6 & 1.737011000 & 19.264249000 & 11.207662000 \\ 6 & 0.447349000 & 19.238557000 & 10.643639000 \\ 6 & 0.205748000 & 18.832115000 & 9.325095000 \\ 6 & 1.308699000 & 18.434419000 & 8.550713000 \\ 6 & 5.492435000 & 17.990361000 & 9.051227000 \\ 6 & 6.335197000 & 19.102163000 & 8.919866000 \\ 6 & 7.441241000 & 19.260789000 & 9.769896000 \\ 6 & 7.666678000 & 18.272804000 & 10.740621000 \\ 6 & 6.843296000 & 17.145071000 & 10.893457000 \\ 6 & 5.751604000 & 17.014609000 & 10.024357000 \\ 6 & 4.586666000 & 15.673345000 & 3.664898000 \\ 6 & 3.316076000 & 15.865359000 & 3.105831000 \\ 6 & 2.954369000 & 15.224208000 & 1.912298000 \\ 6 & 3.909163000 & 14.400538000 & 1.291655000 \\ 6 & 5.194789000 & 14.202015000 & 1.820295000 \\ 6 & 5.519715000 & 14.851749000 & 3.021803000 \\ 6 & 6.788079000 & 16.323172000 & 5.454259000 \\ 6 & 7.615145000 & 17.238126000 & 4.796255000 \\ 6 & 9.012183000 & 17.080223000 & 4.803574000 \\ 6 & 9.539190000 & 15.991465000 & 5.508880000 \\ 6 & 8.733514000 & 15.070940000 & 6.207216000 \\ 6 & 7.345770000 & 15.250068000 & 6.167341000 \\ 6 & 1.922191000 & 19.745820000 & 12.657597000 \\ 6 & 3.393540000 & 19.709815000 & 13.103020000 \\ 6 & 1.416636000 & 21.201279000 & 12.779793000 \\ 6 & 1.106757000 & 18.840775000 & 13.608352000 \\ 6 & -1.198371000 & 18.828167000 & 8.696684000 \\ 6 & -2.290357000 & 19.240790000 & 9.697843000 \\ 6 & -1.527149000 & 17.408801000 & 8.181721000 \\ 6 & -1.222337000 & 19.819247000 & 7.510691000 \\ 6 & 8.395515000 & 20.462924000 & 9.662307000\end{array}$




$\begin{array}{cccc}6 & 8.427704000 & 21.216796000 & 11.011030000 \\ 6 & 9.816187000 & 19.954918000 & 9.328055000 \\ 6 & 7.964255000 & 21.453207000 & 8.568008000 \\ 6 & 7.146678000 & 16.122740000 & 12.003593000 \\ 6 & 7.091849000 & 16.833676000 & 13.375031000 \\ 6 & 8.557863000 & 15.531447000 & 11.794236000 \\ 6 & 6.136601000 & 14.963214000 & 12.022363000 \\ 6 & 1.551291000 & 15.458119000 & 1.325800000 \\ 6 & 1.320216000 & 14.673142000 & 0.023595000 \\ 6 & 0.488120000 & 15.013635000 & 2.355710000 \\ 6 & 1.371137000 & 16.963820000 & 1.026302000 \\ 6 & 6.244964000 & 13.309796000 & 1.134551000 \\ 6 & 5.728578000 & 12.697671000 & -0.178276000 \\ 6 & 6.635330000 & 12.155591000 & 2.085290000 \\ 6 & 7.500580000 & 14.151979000 & 0.814168000 \\ 6 & 9.394068000 & 13.905017000 & 6.962764000 \\ 6 & 8.367794000 & 13.041478000 & 7.715533000 \\ 6 & 10.138974000 & 13.002494000 & 5.952619000 \\ 6 & 10.405650000 & 14.460493000 & 7.989817000 \\ 6 & 9.893226000 & 18.093369000 & 4.052974000 \\ 6 & 11.387511000 & 17.738726000 & 4.128210000 \\ 6 & 9.478890000 & 18.127131000 & 2.564151000 \\ 6 & 9.690630000 & 19.496192000 & 4.668365000 \\ 1 & 2.605566000 & 15.781892000 & 9.573387000 \\ 1 & 2.256516000 & 13.352302000 & 9.198588000 \\ 1 & 3.097676000 & 12.280715000 & 7.105213000 \\ 1 & 4.267134000 & 13.649851000 & 5.396315000 \\ 1 & 3.824324000 & 18.848298000 & 10.813357000 \\ 1 & -0.394728000 & 19.557438000 & 11.258385000 \\ 1 & 1.164445000 & 18.143969000 & 7.507901000 \\ 1 & 6.128117000 & 19.835263000 & 8.139043000 \\ 1 & 8.524141000 & 18.385020000 & 11.408319000 \\ 1 & 5.090278000 & 16.152755000 & 10.105116000 \\ 1 & 2.613426000 & 16.536431000 & 3.605370000 \\ 1 & 3.644228000 & 13.904379000 & 0.359820000 \\ 1 & 6.510351000 & 14.725276000 & 3.461398000 \\ 1 & 7.162917000 & 18.086684000 & 4.278390000 \\ 1 & 10.620164000 & 15.850791000 & 5.525401000 \\ 1 & 6.686127000 & 14.556138000 & 6.687296000 \\ 1 & 3.808358000 & 18.690977000 & 13.067054000 \\ 1 & 3.470942000 & 20.062834000 & 14.142171000 \\ 1 & 4.027171000 & 20.360904000 & 12.482336000 \\ 1 & 1.981378000 & 21.869956000 & 12.113260000 \\ 1 & 1.541174000 & 21.560414000 & 13.813196000 \\ 1 & 0.351675000 & 21.286984000 & 12.520221000 \\ 1 & 0.035009000 & 18.851800000 & 13.363488000 \\ 1 & 1.217831000 & 19.185123000 & 14.648367000 \\ 1 & 1.455553000 & 17.798367000 & 13.554017000\end{array}$




\begin{tabular}{lccc}
1 & -2.337121000 & 18.559129000 & 10.561082000 \\
1 & -3.270905000 & 19.213116000 & 9.199717000 \\
1 & -2.140053000 & 20.264792000 & 10.071827000 \\
1 & -0.812557000 & 17.076369000 & 7.415639000 \\
1 & -2.531795000 & 17.394579000 & 7.731353000 \\
1 & -1.510341000 & 16.677411000 & 9.004158000 \\
1 & -1.005891000 & 20.842884000 & 7.851501000 \\
1 & -2.217225000 & 19.818229000 & 7.038387000 \\
1 & -0.481295000 & 19.556298000 & 6.742257000 \\
1 & 7.427200000 & 21.590529000 & 11.276272000 \\
1 & 9.108125000 & 22.079642000 & 10.943187000 \\
1 & 8.779626000 & 20.576801000 & 11.833038000 \\
1 & 10.200488000 & 19.276182000 & 10.103325000 \\
1 & 10.511897000 & 20.804713000 & 9.248228000 \\
1 & 9.822802000 & 19.414070000 & 8.369997000 \\
1 & 7.944814000 & 20.989156000 & 7.571414000 \\
1 & 8.676370000 & 22.291296000 & 8.532658000 \\
1 & 6.966705000 & 21.873207000 & 8.765642000 \\
1 & 7.830484000 & 17.645079000 & 13.444362000 \\
1 & 7.303664000 & 16.116050000 & 14.182820000 \\
1 & 6.096489000 & 17.267793000 & 13.553786000 \\
1 & 8.622851000 & 15.001731000 & 10.832394000 \\
1 & 8.791177000 & 14.814026000 & 12.596335000 \\
1 & 9.333640000 & 16.310325000 & 11.804076000 \\
1 & 5.111324000 & 15.315509000 & 12.211605000 \\
1 & 6.400155000 & 14.261639000 & 12.827639000 \\
1 & 6.139731000 & 14.400626000 & 11.076402000 \\
1 & 2.026410000 & 14.970915000 & -0.766189000 \\
1 & 0.304603000 & 14.874802000 & -0.348060000 \\
1 & 1.408043000 & 13.586532000 & 0.176167000 \\
1 & 0.591298000 & 13.943382000 & 2.591078000 \\
1 & -0.522438000 & 15.179927000 & 1.951171000 \\
1 & 0.571071000 & 15.578880000 & 3.294656000 \\
1 & 1.483821000 & 17.577038000 & 1.931521000 \\
1 & 0.366391000 & 17.149136000 & 0.615367000 \\
1 & 2.111969000 & 17.308313000 & 0.289147000 \\
1 & 4.849089000 & 12.056583000 & -0.015187000 \\
1 & 6.516202000 & 12.073213000 & -0.625727000 \\
1 & 5.460808000 & 13.471378000 & -0.913471000 \\
1 & 7.058234000 & 12.531228000 & 3.028164000 \\
1 & 7.391550000 & 11.511547000 & 1.609942000 \\
1 & 5.760418000 & 11.533760000 & 2.328481000 \\
1 & 7.254541000 & 14.981040000 & 0.133983000 \\
1 & 8.263676000 & 13.524069000 & 0.328269000 \\
& 7.946301000 & 14.582200000 & 1.722390000 \\
\hline & 8.806188000 & 13.627856000 & 8.458661000 \\
\hline
\end{tabular}




$\begin{array}{cccc}1 & 9.442610000 & 12.583863000 & 5.210116000 \\ 1 & 10.624283000 & 12.164600000 & 6.477005000 \\ 1 & 10.918338000 & 13.556484000 & 5.409588000 \\ 1 & 11.202973000 & 15.043334000 & 7.506888000 \\ 1 & 10.881165000 & 13.632338000 & 8.537730000 \\ 1 & 9.906132000 & 15.114919000 & 8.719453000 \\ 1 & 11.757041000 & 17.732861000 & 5.164857000 \\ 1 & 11.969651000 & 18.489853000 & 3.574078000 \\ 1 & 11.599718000 & 16.756510000 & 3.678659000 \\ 1 & 9.604390000 & 17.139094000 & 2.095408000 \\ 1 & 10.104531000 & 18.848487000 & 2.016013000 \\ 1 & 8.430259000 & 18.431109000 & 2.438517000 \\ 1 & 8.640830000 & 19.818370000 & 4.620119000 \\ 1 & 10.298758000 & 20.237065000 & 4.126223000 \\ 1 & 9.998663000 & 19.508192000 & 5.724937000\end{array}$

Energy $=-5278.051650$ A.U. 


\subsection{2 $\mathrm{FeCl}_{2}(\mathrm{dpbz})$ optimized with uPBEPBE/TZVP (toluene PCM)}

$\begin{array}{llll}26 & 2.479975000 & 7.459875000 & 3.755326000 \\ 17 & 1.656390000 & 9.162622000 & 2.549976000 \\ 17 & 1.238534000 & 6.184538000 & 5.089910000 \\ 15 & 3.994621000 & 6.275499000 & 2.299654000 \\ 15 & 4.549407000 & 8.230564000 & 4.721576000 \\ 6 & 5.466720000 & 7.403814000 & 2.193664000 \\ 6 & 6.300528000 & 7.443741000 & 1.067295000 \\ 1 & 6.093814000 & 6.786394000 & 0.220400000 \\ 6 & 7.375952000 & 8.333805000 & 1.013242000 \\ 1 & 8.015028000 & 8.359223000 & 0.128494000 \\ 6 & 7.622472000 & 9.198132000 & 2.083732000 \\ 1 & 8.455474000 & 9.902461000 & 2.040245000 \\ 6 & 6.793595000 & 9.173044000 & 3.208125000 \\ 1 & 6.973636000 & 9.866162000 & 4.032388000 \\ 6 & 5.715614000 & 8.278755000 & 3.276974000 \\ 6 & 3.596833000 & 5.890935000 & 0.533359000 \\ 6 & 4.005336000 & 4.703579000 & -0.093738000 \\ 1 & 4.580981000 & 3.960312000 & 0.460743000 \\ 6 & 3.670708000 & 4.467340000 & -1.430785000 \\ 1 & 3.990117000 & 3.540243000 & -1.911221000 \\ 6 & 2.929286000 & 5.410633000 & -2.147665000 \\ 1 & 2.668046000 & 5.222870000 & -3.190991000 \\ 6 & 2.513479000 & 6.590998000 & -1.522300000 \\ 1 & 1.923010000 & 7.325424000 & -2.073596000 \\ 6 & 2.835877000 & 6.829857000 & -0.184783000 \\ 1 & 2.488943000 & 7.741613000 & 0.308037000 \\ 6 & 4.652747000 & 4.688512000 & 2.979207000 \\ 6 & 6.016354000 & 4.358077000 & 2.952986000 \\ 1 & 6.743701000 & 5.059728000 & 2.540394000 \\ 6 & 6.449205000 & 3.130217000 & 3.461398000 \\ 1 & 7.512308000 & 2.881694000 & 3.440851000 \\ 6 & 5.526822000 & 2.225511000 & 3.995003000 \\ 1 & 5.867822000 & 1.267385000 & 4.392168000 \\ 6 & 4.167959000 & 2.553987000 & 4.027681000 \\ 1 & 3.444207000 & 1.856802000 & 4.454109000 \\ 6 & 3.730084000 & 3.782758000 & 3.528851000 \\ 1 & 2.671921000 & 4.047377000 & 3.584382000 \\ 6 & 5.328904000 & 7.068593000 & 5.928630000 \\ 6 & 6.693901000 & 6.744355000 & 5.894378000 \\ 1 & 7.337800000 & 7.163561000 & 5.119038000 \\ 6 & 7.232359000 & 5.878811000 & 6.850673000 \\ 1 & 8.294786000 & 5.628996000 & 6.816215000 \\ 6 & 6.414381000 & 5.333326000 & 7.844415000 \\ 1 & 6.836911000 & 4.656059000 & 8.589305000 \\ 6 & 5.052681000 & 5.649282000 & 7.877368000 \\ 1 & 4.406756000 & 5.216403000 & 8.643603000\end{array}$




$\begin{array}{lccc}6 & 4.507192000 & 6.508456000 & 6.920833000 \\ 1 & 3.437420000 & 6.727977000 & 6.932081000 \\ 6 & 4.727968000 & 9.898281000 & 5.502904000 \\ 6 & 4.015956000 & 10.967363000 & 4.931295000 \\ 1 & 3.377619000 & 10.793772000 & 4.061300000 \\ 6 & 4.114348000 & 12.246278000 & 5.482969000 \\ 1 & 3.558712000 & 13.071396000 & 5.033069000 \\ 6 & 4.905504000 & 12.466402000 & 6.615678000 \\ 1 & 4.972914000 & 13.465695000 & 7.050260000 \\ 6 & 5.601276000 & 11.402124000 & 7.195779000 \\ 1 & 6.213799000 & 11.566384000 & 8.084698000 \\ 6 & 5.515357000 & 10.120342000 & 6.643296000 \\ 1 & 6.058954000 & 9.293659000 & 7.104123000\end{array}$

Energy $=-4022.254510$ A.U. 


\subsection{3 $\mathrm{FeCl}_{2}\left({ }^{\mathrm{tBu}} \mathrm{dppe}\right)$ optimized with uPBEPBE/TZVP (toluene PCM)}

$\begin{array}{lccc}26 & 8.613986000 & 6.905554000 & 8.682715000 \\ 17 & 10.832528000 & 7.137466000 & 8.774611000 \\ 17 & 7.642200000 & 6.513431000 & 6.702745000 \\ 15 & 7.597772000 & 8.664264000 & 10.045668000 \\ 15 & 7.824612000 & 5.405310000 & 10.434155000 \\ 6 & 6.731389000 & 6.456864000 & 11.539482000 \\ 1 & 5.760572000 & 6.510970000 & 11.022121000 \\ 1 & 6.548663000 & 5.961525000 & 12.504815000 \\ 6 & 7.326410000 & 7.855516000 & 11.720461000 \\ 1 & 8.317507000 & 7.808861000 & 12.198189000 \\ 1 & 6.690163000 & 8.485426000 & 12.359520000 \\ 6 & 8.523119000 & 10.206460000 & 10.483329000 \\ 6 & 7.925802000 & 11.471864000 & 10.466500000 \\ 1 & 6.877808000 & 11.563438000 & 10.178138000 \\ 6 & 8.660452000 & 12.617423000 & 10.811297000 \\ 6 & 10.006332000 & 12.453108000 & 11.174203000 \\ 1 & 10.587053000 & 13.332404000 & 11.445509000 \\ 6 & 10.636931000 & 11.197420000 & 11.188951000 \\ 6 & 9.875744000 & 10.076300000 & 10.829117000 \\ 1 & 10.345341000 & 9.091008000 & 10.785544000 \\ 6 & 5.913778000 & 9.232101000 & 9.533292000 \\ 6 & 5.621747000 & 9.255246000 & 8.167215000 \\ 1 & 6.366731000 & 8.882604000 & 7.461475000 \\ 6 & 4.379667000 & 9.725646000 & 7.699854000 \\ 6 & 3.447733000 & 10.154486000 & 8.650966000 \\ 1 & 2.477829000 & 10.517023000 & 8.309660000 \\ 6 & 3.704901000 & 10.134726000 & 10.036520000 \\ 6 & 4.953586000 & 9.671531000 & 10.462901000 \\ 1 & 5.188378000 & 9.660617000 & 11.526672000 \\ 6 & 9.158475000 & 4.757891000 & 11.541030000 \\ 6 & 10.269236000 & 4.181193000 & 10.917861000 \\ 1 & 10.331925000 & 4.199160000 & 9.827921000 \\ 6 & 11.304611000 & 3.602702000 & 11.672086000 \\ 6 & 11.193337000 & 3.643472000 & 13.067327000 \\ 1 & 11.988486000 & 3.205707000 & 13.671550000 \\ 6 & 10.100433000 & 4.235754000 & 13.728239000 \\ 6 & 9.082067000 & 4.789308000 & 12.943439000 \\ 1 & 8.222360000 & 5.248420000 & 13.429016000 \\ 6 & 6.755272000 & 3.934471000 & 10.090053000 \\ 6 & 6.591338000 & 2.910518000 & 11.038457000 \\ 1 & 7.145669000 & 2.961718000 & 11.975577000 \\ 6 & 5.742861000 & 1.830804000 & 10.775476000 \\ 6 & 5.065748000 & 1.808664000 & 9.538946000 \\ 1 & 4.405403000 & 0.967042000 & 9.326524000 \\ 6 & 5.213751000 & 2.810594000 & 8.573696000 \\ 6 & 6.079967000 & 3.880475000 & 8.868923000\end{array}$




\begin{tabular}{lccc}
1 & 6.247668000 & 4.672444000 & 8.134938000 \\
6 & 12.121427000 & 11.019285000 & 11.551640000 \\
6 & 12.780616000 & 12.336836000 & 11.993449000 \\
1 & 12.288927000 & 12.766573000 & 12.879750000 \\
1 & 12.771247000 & 13.091335000 & 11.192417000 \\
1 & 13.832492000 & 12.148227000 & 12.254935000 \\
6 & 12.253131000 & 10.003601000 & 12.709056000 \\
1 & 11.717645000 & 10.354385000 & 13.604491000 \\
1 & 13.313442000 & 9.870208000 & 12.973999000 \\
1 & 11.852104000 & 9.017504000 & 12.435292000 \\
6 & 12.879335000 & 10.483881000 & 10.315404000 \\
1 & 13.943737000 & 10.338398000 & 10.558421000 \\
1 & 12.813036000 & 11.194977000 & 9.478269000 \\
1 & 12.473730000 & 9.521517000 & 9.971690000 \\
6 & 7.971052000 & 13.993344000 & 10.781122000 \\
6 & 8.922662000 & 15.136415000 & 11.173359000 \\
1 & 9.777553000 & 15.213150000 & 10.484772000 \\
1 & 9.311278000 & 15.016041000 & 12.195887000 \\
1 & 8.380118000 & 16.092865000 & 11.135288000 \\
6 & 7.445208000 & 14.271135000 & 9.354911000 \\
1 & 8.270054000 & 14.277857000 & 8.626895000 \\
1 & 6.947392000 & 15.252770000 & 9.319100000 \\
1 & 6.717517000 & 13.512463000 & 9.033079000 \\
6 & 6.783749000 & 13.992047000 & 11.770546000 \\
1 & 7.128026000 & 13.797676000 & 12.797611000 \\
1 & 6.040151000 & 13.224342000 & 11.511010000 \\
1 & 6.277572000 & 14.969973000 & 11.757456000 \\
6 & 4.091330000 & 9.732982000 & 6.189663000 \\
6 & 2.722709000 & 10.350570000 & 5.858214000 \\
1 & 1.894360000 & 9.780059000 & 6.305132000 \\
1 & 2.574690000 & 10.346089000 & 4.768209000 \\
1 & 2.649145000 & 11.394922000 & 6.198577000 \\
6 & 5.181408000 & 10.551516000 & 5.461684000 \\
1 & 6.182605000 & 10.124123000 & 5.611533000 \\
1 & 5.197582000 & 11.592749000 & 5.817893000 \\
1 & 4.981938000 & 10.562398000 & 4.379049000 \\
6 & 4.111905000 & 8.278711000 & 5.666094000 \\
1 & 3.339728000 & 7.672053000 & 6.163627000 \\
1 & 5.085154000 & 7.796098000 & 5.835549000 \\
1 & 3.911480000 & 8.266309000 & 4.583333000 \\
6 & 2.622733000 & 10.620018000 & 11.016850000 \\
6 & 3.075144000 & 10.524200000 & 12.483808000 \\
1 & 3.959150000 & 11.148959000 & 12.682756000 \\
1 & 3.308134000 & 9.488534000 & 12.774478000 \\
& 2.266251000 & 10.877837000 & 13.140101000 \\
& 2.279400000 & 12.096009000 & 10.712964000 \\
\hline & 1.497953000 & 12.452556000 & 11.401858000
\end{tabular}




$\begin{array}{lccc}1 & 1.906958000 & 12.226191000 & 9.686866000 \\ 6 & 1.353450000 & 9.755419000 & 10.844680000 \\ 1 & 1.567014000 & 8.696689000 & 11.054679000 \\ 1 & 0.949456000 & 9.822029000 & 9.824435000 \\ 1 & 0.568705000 & 10.092061000 & 11.539794000 \\ 6 & 4.487293000 & 2.778340000 & 7.219038000 \\ 6 & 3.605102000 & 4.040131000 & 7.084671000 \\ 1 & 4.202724000 & 4.960926000 & 7.140890000 \\ 1 & 2.845495000 & 4.075567000 & 7.880475000 \\ 1 & 3.085510000 & 4.035835000 & 6.113811000 \\ 6 & 3.589480000 & 1.539678000 & 7.065102000 \\ 1 & 4.167470000 & 0.604436000 & 7.116860000 \\ 1 & 3.093748000 & 1.567958000 & 6.083436000 \\ 1 & 2.802374000 & 1.504245000 & 7.833807000 \\ 6 & 5.534012000 & 2.767439000 & 6.081455000 \\ 1 & 6.182433000 & 1.881163000 & 6.152902000 \\ 1 & 6.174073000 & 3.660743000 & 6.106304000 \\ 1 & 5.027320000 & 2.743202000 & 5.103941000 \\ 6 & 5.533355000 & 0.679685000 & 11.774058000 \\ 6 & 5.974360000 & -0.649635000 & 11.120783000 \\ 1 & 5.397197000 & -0.869150000 & 10.211077000 \\ 1 & 5.826577000 & -1.485147000 & 11.822725000 \\ 1 & 7.039098000 & -0.616873000 & 10.845411000 \\ 6 & 4.037170000 & 0.592751000 & 12.150645000 \\ 1 & 3.403042000 & 0.417220000 & 11.269831000 \\ 1 & 3.698555000 & 1.523324000 & 12.630638000 \\ 1 & 3.870821000 & -0.236954000 & 12.855280000 \\ 6 & 6.344510000 & 0.871330000 & 13.066626000 \\ 1 & 7.426588000 & 0.909364000 & 12.870381000 \\ 1 & 6.158779000 & 0.024855000 & 13.744408000 \\ 1 & 6.056637000 & 1.791168000 & 13.598199000 \\ 6 & 10.061407000 & 4.252942000 & 15.266339000 \\ 6 & 11.289669000 & 5.026059000 & 15.797554000 \\ 1 & 11.278408000 & 5.048483000 & 16.898404000 \\ 1 & 11.286591000 & 6.064340000 & 15.432916000 \\ 1 & 12.233260000 & 4.560202000 & 15.479913000 \\ 6 & 8.794225000 & 4.931438000 & 15.814046000 \\ 1 & 7.878770000 & 4.407332000 & 15.499561000 \\ 1 & 8.721093000 & 5.982242000 & 15.495243000 \\ 1 & 8.819462000 & 4.920495000 & 16.913778000 \\ 6 & 10.098740000 & 2.801820000 & 15.796741000 \\ 1 & 9.230960000 & 2.229246000 & 15.435315000 \\ 1 & 10.077325000 & 2.799494000 & 16.897615000 \\ 1 & 11.008147000 & 2.273132000 & 15.477238000 \\ 6 & 12.515802000 & 2.987524000 & 10.951022000 \\ 6 & 13.275876000 & 4.110028000 & 10.208978000 \\ 1 & 12.631416000 & 4.632905000 & 9.488494000 \\ 1 & 14.132841000 & 3.687754000 & 9.661097000\end{array}$




$\begin{array}{llll}1 & 13.657948000 & 4.858246000 & 10.919737000 \\ 6 & 12.031155000 & 1.934655000 & 9.929479000 \\ 1 & 11.380702000 & 2.375923000 & 9.161360000 \\ 1 & 11.470325000 & 1.129460000 & 10.427888000 \\ 1 & 12.894104000 & 1.484155000 & 9.415599000 \\ 6 & 13.489453000 & 2.298580000 & 11.922248000 \\ 1 & 13.921981000 & 3.005985000 & 12.645951000 \\ 1 & 14.323522000 & 1.862889000 & 11.352507000 \\ 1 & 13.004550000 & 1.482749000 & 12.480140000\end{array}$

Energy $=-5125.787596$ A.U. 


\subsection{4 $\mathrm{FeCl}_{2}$ (Xantphos) optimized with uPBEPBE/TZVP (gas phase calculation)}

$\begin{array}{lccc}26 & 5.559365000 & 3.569867000 & 4.082921000 \\ 17 & 6.572323000 & 3.556117000 & 2.097988000 \\ 17 & 5.535374000 & 1.976636000 & 5.644631000 \\ 15 & 6.464057000 & 5.525131000 & 5.239168000 \\ 15 & 3.199254000 & 3.965957000 & 3.592537000 \\ 8 & 3.635057000 & 5.561615000 & 6.009126000 \\ 6 & 5.797532000 & 5.808081000 & 6.945597000 \\ 6 & 6.608188000 & 5.985406000 & 8.075109000 \\ 1 & 7.691823000 & 6.028165000 & 7.958485000 \\ 6 & 6.036012000 & 6.077533000 & 9.345141000 \\ 1 & 6.677027000 & 6.205918000 & 10.219005000 \\ 6 & 4.650384000 & 5.994774000 & 9.506182000 \\ 1 & 4.226034000 & 6.062301000 & 10.508607000 \\ 6 & 0.638376000 & 4.046578000 & 7.484065000 \\ 1 & 0.064028000 & 4.037629000 & 8.411125000 \\ 6 & 0.216352000 & 3.256235000 & 6.411633000 \\ 1 & -0.678086000 & 2.638980000 & 6.511621000 \\ 6 & 0.936389000 & 3.242882000 & 5.215558000 \\ 1 & 0.613833000 & 2.608266000 & 4.389104000 \\ 6 & 2.094067000 & 4.020537000 & 5.079437000 \\ 6 & 2.274222000 & 5.810976000 & 8.469039000 \\ 6 & 1.752918000 & 7.236020000 & 8.129924000 \\ 1 & 2.095141000 & 7.562959000 & 7.138197000 \\ 1 & 2.115868000 & 7.959229000 & 8.875649000 \\ 1 & 0.652636000 & 7.247615000 & 8.135403000 \\ 6 & 1.763536000 & 5.417352000 & 9.861622000 \\ 1 & 2.106058000 & 4.414505000 & 10.153922000 \\ 1 & 0.665347000 & 5.433444000 & 9.890430000 \\ 1 & 2.105184000 & 6.137731000 & 10.617675000 \\ 6 & 4.409686000 & 5.738885000 & 7.141847000 \\ 6 & 3.804647000 & 5.832349000 & 8.402273000 \\ 6 & 1.780235000 & 4.850484000 & 7.382264000 \\ 6 & 2.485319000 & 4.808886000 & 6.172442000 \\ 6 & 8.287358000 & 5.362850000 & 5.536549000 \\ 6 & 9.158291000 & 6.462912000 & 5.519675000 \\ 1 & 8.769718000 & 7.462784000 & 5.317870000 \\ 6 & 10.524343000 & 6.279452000 & 5.751388000 \\ 1 & 11.196483000 & 7.139944000 & 5.731681000 \\ 6 & 11.028733000 & 5.000087000 & 6.002025000 \\ 1 & 12.096931000 & 4.858404000 & 6.178789000 \\ 6 & 10.164259000 & 3.901462000 & 6.017655000 \\ 1 & 10.553743000 & 2.898729000 & 6.204216000 \\ 6 & 8.799364000 & 4.077397000 & 5.779068000 \\ 1 & 8.127851000 & 3.214673000 & 5.781461000 \\ 6 & 6.318552000 & 7.171552000 & 4.405795000 \\ 6 & 5.871483000 & 8.322317000 & 5.070770000\end{array}$




$\begin{array}{lccc}1 & 5.592127000 & 8.270078000 & 6.124733000 \\ 6 & 5.789741000 & 9.540439000 & 4.389324000 \\ 1 & 5.439170000 & 10.430916000 & 4.915490000 \\ 6 & 6.160568000 & 9.620006000 & 3.044781000 \\ 1 & 6.100163000 & 10.573076000 & 2.515419000 \\ 6 & 6.607223000 & 8.474036000 & 2.378945000 \\ 1 & 6.894218000 & 8.527968000 & 1.326725000 \\ 6 & 6.678436000 & 7.252033000 & 3.049531000 \\ 1 & 7.009756000 & 6.355392000 & 2.519221000 \\ 6 & 2.749839000 & 5.483834000 & 2.633948000 \\ 6 & 1.668486000 & 6.304251000 & 2.986165000 \\ 1 & 1.058722000 & 6.057547000 & 3.857198000 \\ 6 & 1.365711000 & 7.434718000 & 2.221651000 \\ 1 & 0.522977000 & 8.068909000 & 2.505102000 \\ 6 & 2.134226000 & 7.748932000 & 1.098036000 \\ 1 & 1.894898000 & 8.630637000 & 0.500040000 \\ 6 & 3.213283000 & 6.932716000 & 0.744208000 \\ 1 & 3.822018000 & 7.176588000 & -0.128885000 \\ 6 & 3.528981000 & 5.809532000 & 1.510919000 \\ 1 & 4.385275000 & 5.184997000 & 1.242727000 \\ 6 & 2.465504000 & 2.588961000 & 2.592151000 \\ 6 & 1.509807000 & 2.803497000 & 1.587559000 \\ 1 & 1.168837000 & 3.816196000 & 1.364825000 \\ 6 & 0.998444000 & 1.722197000 & 0.864056000 \\ 1 & 0.258760000 & 1.898199000 & 0.080135000 \\ 6 & 1.433850000 & 0.422997000 & 1.139460000 \\ 1 & 1.035061000 & -0.419589000 & 0.570841000 \\ 6 & 2.387308000 & 0.205703000 & 2.138913000 \\ 1 & 2.738313000 & -0.805778000 & 2.352643000 \\ 6 & 2.909189000 & 1.282616000 & 2.858500000 \\ 1 & 3.663808000 & 1.110288000 & 3.630615000\end{array}$

Energy $=-4444.728015$ A.U. 


\subsubsection{FeCl(dppe) ${ }_{2}$ optimized with uPBEPBE/TZVP (toluene PCM)}

$\begin{array}{lrrr}26 & 2.737266000 & 17.791530000 & 5.350174000 \\ 17 & 2.876994000 & 17.807051000 & 3.011086000 \\ 15 & 1.293058000 & 16.641329000 & 6.609276000 \\ 15 & 3.902314000 & 15.859706000 & 5.439797000 \\ 15 & 1.577824000 & 19.723811000 & 5.305749000 \\ 15 & 4.012482000 & 18.940111000 & 6.789346000 \\ 6 & 2.189522000 & 15.263597000 & 7.586594000 \\ 1 & 2.128480000 & 15.482502000 & 8.660097000 \\ 1 & 1.624955000 & 14.336639000 & 7.415625000 \\ 6 & 3.651270000 & 15.126291000 & 7.146102000 \\ 1 & 3.989763000 & 14.081243000 & 7.188162000 \\ 1 & 4.306481000 & 15.717128000 & 7.800019000 \\ 6 & 0.048789000 & 15.583426000 & 5.674981000 \\ 6 & 0.154646000 & 15.490560000 & 4.281871000 \\ 1 & 0.934302000 & 16.060259000 & 3.767825000 \\ 6 & -0.708761000 & 14.662118000 & 3.556412000 \\ 1 & -0.609042000 & 14.597590000 & 2.470700000 \\ 6 & -1.685170000 & 13.914848000 & 4.218908000 \\ 1 & -2.359850000 & 13.267632000 & 3.654251000 \\ 6 & -1.792776000 & 13.994226000 & 5.612673000 \\ 1 & -2.552525000 & 13.410675000 & 6.137816000 \\ 6 & -0.930870000 & 14.822076000 & 6.336024000 \\ 1 & -1.030534000 & 14.881204000 & 7.422579000 \\ 6 & 0.159771000 & 17.364999000 & 7.912420000 \\ 6 & 0.582932000 & 17.557687000 & 9.238811000 \\ 1 & 1.567634000 & 17.212421000 & 9.556927000 \\ 6 & -0.243456000 & 18.181855000 & 10.178204000 \\ 1 & 0.106338000 & 18.309323000 & 11.205148000 \\ 6 & -1.513259000 & 18.633806000 & 9.807113000 \\ 1 & -2.161163000 & 19.119460000 & 10.539389000 \\ 6 & -1.945345000 & 18.454363000 & 8.489593000 \\ 1 & -2.934352000 & 18.802614000 & 8.184186000 \\ 6 & -1.119373000 & 17.828555000 & 7.552182000 \\ 1 & -1.478324000 & 17.697359000 & 6.530286000 \\ 6 & 3.469878000 & 14.441606000 & 4.296074000 \\ 6 & 2.841143000 & 13.273503000 & 4.754299000 \\ 1 & 2.570137000 & 13.161453000 & 5.804638000 \\ 6 & 2.543392000 & 12.228429000 & 3.873877000 \\ 1 & 2.054553000 & 11.328185000 & 4.252506000 \\ 6 & 2.866867000 & 12.336767000 & 2.519705000 \\ 1 & 2.635935000 & 11.520699000 & 1.831844000 \\ 6 & 3.489792000 & 13.498434000 & 2.052133000 \\ 1 & 3.746733000 & 13.595237000 & 0.995009000 \\ 6 & 3.789244000 & 14.542016000 & 2.930298000 \\ 1 & 4.263418000 & 15.446655000 & 2.550037000 \\ 6 & 5.750374000 & 15.830569000 & 5.200219000\end{array}$




\begin{tabular}{llll}
6 & 6.581064000 & 14.934667000 & 5.892231000 \\
1 & 6.161567000 & 14.239969000 & 6.621611000 \\
6 & 7.958519000 & 14.923280000 & 5.656501000 \\
1 & 8.595105000 & 14.226371000 & 6.206200000 \\
6 & 8.519808000 & 15.796714000 & 4.719195000 \\
1 & 9.596197000 & 15.785343000 & 4.535587000 \\
6 & 7.696012000 & 16.679875000 & 4.015367000 \\
1 & 8.125232000 & 17.362884000 & 3.279269000 \\
6 & 6.318920000 & 16.698255000 & 4.255699000 \\
1 & 5.674790000 & 17.388528000 & 3.708234000 \\
6 & 1.602688000 & 20.461017000 & 7.027348000 \\
1 & 1.316089000 & 21.521855000 & 7.007275000 \\
1 & 0.822088000 & 19.917182000 & 7.575766000 \\
6 & 2.969494000 & 20.259624000 & 7.696373000 \\
1 & 2.836761000 & 19.945017000 & 8.738809000 \\
1 & 3.557185000 & 21.187827000 & 7.711691000 \\
6 & -0.224597000 & 19.765377000 & 4.831132000 \\
6 & -1.122201000 & 20.686933000 & 5.394275000 \\
1 & -0.785801000 & 21.397329000 & 6.151295000 \\
6 & -2.461025000 & 20.704504000 & 4.994361000 \\
1 & -3.150310000 & 21.421512000 & 5.445980000 \\
6 & -2.916596000 & 19.811359000 & 4.018797000 \\
1 & -3.963057000 & 19.826999000 & 3.707182000 \\
6 & -2.024433000 & 18.903080000 & 3.442345000 \\
1 & -2.369576000 & 18.203473000 & 2.678147000 \\
6 & -0.686194000 & 18.878854000 & 3.847056000 \\
1 & 0.008364000 & 18.166692000 & 3.398377000 \\
6 & 2.162097000 & 21.143797000 & 4.233107000 \\
6 & 2.789396000 & 22.277150000 & 4.773174000 \\
1 & 2.965158000 & 22.358960000 & 5.846468000 \\
6 & 3.212412000 & 23.323189000 & 3.947044000 \\
1 & 3.698111000 & 24.195797000 & 4.389076000 \\
6 & 3.018291000 & 23.249733000 & 2.566035000 \\
1 & 3.347177000 & 24.066446000 & 1.920032000 \\
6 & 2.398835000 & 22.122212000 & 2.017192000 \\
1 & 2.242786000 & 22.053253000 & 0.938406000 \\
6 & 1.973935000 & 21.078000000 & 2.841371000 \\
1 & 1.501681000 & 20.200857000 & 2.399211000 \\
6 & 5.330241000 & 20.041973000 & 6.022570000 \\
6 & 5.449102000 & 20.089491000 & 4.628504000 \\
1 & 4.783922000 & 19.476202000 & 4.014324000 \\
6 & 6.388632000 & 20.930989000 & 4.022868000 \\
1 & 6.464538000 & 20.959468000 & 2.933741000 \\
6 & 7.215902000 & 21.738269000 & 4.806549000 \\
6 & 7.949145000 & 22.396323000 & 4.335183000 \\
\hline & 7.749895000 & 21.704703000 & 6.201521000 \\
\hline & 6.162213000 & 20.862099000 & 6.805118000
\end{tabular}




$\begin{array}{llll}1 & 6.089702000 & 20.836048000 & 7.895313000 \\ 6 & 5.015064000 & 18.224437000 & 8.200218000 \\ 6 & 6.346969000 & 17.829135000 & 7.979819000 \\ 1 & 6.810008000 & 18.009609000 & 7.008917000 \\ 6 & 7.090124000 & 17.214913000 & 8.991143000 \\ 1 & 8.123022000 & 16.920026000 & 8.794162000 \\ 6 & 6.518860000 & 16.978663000 & 10.245289000 \\ 1 & 7.101442000 & 16.502119000 & 11.036162000 \\ 6 & 5.194472000 & 17.360934000 & 10.477318000 \\ 1 & 4.735933000 & 17.187122000 & 11.453411000 \\ 6 & 4.451205000 & 17.972655000 & 9.463256000 \\ 1 & 3.419727000 & 18.258898000 & 9.673140000\end{array}$

Energy $=-5096.171316$ A.U. 


\subsubsection{FeCl(dpbz) $)_{2}$ optimized with uPBEPBE/TZVP (toluene PCM)}

$\begin{array}{lccc}26 & 5.064192000 & 2.433568000 & 6.643351000 \\ 15 & 3.478121000 & 1.226898000 & 5.593272000 \\ 17 & 5.177854000 & 2.684415000 & 8.955273000 \\ 6 & 7.363803000 & 1.786741000 & 4.301397000 \\ 6 & 7.931249000 & 1.777911000 & 3.019813000 \\ 1 & 7.848562000 & 2.660550000 & 2.382210000 \\ 6 & 8.591224000 & 0.636639000 & 2.552675000 \\ 1 & 9.018638000 & 0.629053000 & 1.547654000 \\ 6 & 8.709416000 & -0.488285000 & 3.374167000 \\ 1 & 9.227846000 & -1.379060000 & 3.013379000 \\ 6 & 8.158172000 & -0.478959000 & 4.659337000 \\ 1 & 8.253112000 & -1.363910000 & 5.290268000 \\ 6 & 7.466220000 & 0.648063000 & 5.120100000 \\ 6 & 7.895476000 & 4.255955000 & 5.699961000 \\ 6 & 7.622631000 & 5.300764000 & 6.597630000 \\ 1 & 6.597110000 & 5.488381000 & 6.918380000 \\ 6 & 8.654603000 & 6.100625000 & 7.094703000 \\ 1 & 8.419563000 & 6.912714000 & 7.786227000 \\ 6 & 9.979221000 & 5.855786000 & 6.718378000 \\ 1 & 10.787376000 & 6.474778000 & 7.113726000 \\ 6 & 10.262259000 & 4.806695000 & 5.839488000 \\ 1 & 11.294121000 & 4.598610000 & 5.547911000 \\ 6 & 9.228357000 & 4.014406000 & 5.330492000 \\ 1 & 9.468253000 & 3.200317000 & 4.645208000 \\ 15 & 6.453436000 & 3.236343000 & 5.059664000 \\ 15 & 6.673881000 & 0.852844000 & 6.796679000 \\ 6 & 6.049440000 & 4.218673000 & 3.507096000 \\ 6 & 4.966586000 & 3.820038000 & 2.706372000 \\ 1 & 4.352648000 & 2.970625000 & 3.005361000 \\ 6 & 4.644178000 & 4.514224000 & 1.537416000 \\ 1 & 3.796917000 & 4.184707000 & 0.932262000 \\ 6 & 5.394713000 & 5.629917000 & 1.152375000 \\ 1 & 5.140066000 & 6.177377000 & 0.242500000 \\ 6 & 6.471951000 & 6.038310000 & 1.942987000 \\ 1 & 7.066424000 & 6.907815000 & 1.653410000 \\ 6 & 6.798770000 & 5.337962000 & 3.109012000 \\ 1 & 7.642134000 & 5.673396000 & 3.713064000 \\ 15 & 3.473480000 & 4.040623000 & 6.559594000 \\ 6 & 8.209155000 & 1.129496000 & 7.845033000 \\ 6 & 8.439728000 & 2.354387000 & 8.482412000 \\ 1 & 7.686840000 & 3.137864000 & 8.425487000 \\ 6 & 9.615405000 & 2.561995000 & 9.211910000 \\ 1 & 9.776173000 & 3.522298000 & 9.706401000 \\ 6 & 10.574504000 & 1.552023000 & 9.309087000 \\ 1 & 11.491141000 & 1.715994000 & 9.879817000 \\ 6 & 10.351358000 & 0.324361000 & 8.675823000\end{array}$




$\begin{array}{lccc}1 & 11.091480000 & -0.475502000 & 8.749437000 \\ 6 & 9.176492000 & 0.114680000 & 7.951133000 \\ 1 & 9.011038000 & -0.855908000 & 7.480150000 \\ 6 & 6.230243000 & -0.861607000 & 7.364431000 \\ 6 & 6.208273000 & -1.114693000 & 8.748786000 \\ 1 & 6.511532000 & -0.332093000 & 9.446881000 \\ 6 & 5.800121000 & -2.358827000 & 9.233921000 \\ 1 & 5.790685000 & -2.540492000 & 10.310716000 \\ 6 & 5.407339000 & -3.366987000 & 8.346632000 \\ 1 & 5.092115000 & -4.340884000 & 8.726627000 \\ 6 & 5.416734000 & -3.118642000 & 6.971726000 \\ 1 & 5.105982000 & -3.896212000 & 6.270684000 \\ 6 & 5.818198000 & -1.871732000 & 6.481424000 \\ 1 & 5.802043000 & -1.689447000 & 5.406711000 \\ 6 & 2.405942000 & 3.740701000 & 5.058749000 \\ 6 & 1.604588000 & 4.691007000 & 4.413274000 \\ 1 & 1.589455000 & 5.725834000 & 4.758793000 \\ 6 & 0.831687000 & 4.324578000 & 3.307109000 \\ 1 & 0.226149000 & 5.076002000 & 2.796108000 \\ 6 & 0.838948000 & 3.001803000 & 2.854847000 \\ 1 & 0.237839000 & 2.714680000 & 1.989359000 \\ 6 & 1.610465000 & 2.041023000 & 3.516271000 \\ 1 & 1.608672000 & 1.005925000 & 3.168790000 \\ 6 & 2.397469000 & 2.404974000 & 4.617657000 \\ 6 & 3.999459000 & 5.824007000 & 6.489450000 \\ 6 & 4.300713000 & 6.457703000 & 7.710535000 \\ 1 & 4.155013000 & 5.920551000 & 8.650034000 \\ 6 & 4.785499000 & 7.766485000 & 7.728786000 \\ 1 & 5.011259000 & 8.244363000 & 8.684447000 \\ 6 & 4.981027000 & 8.461971000 & 6.529983000 \\ 1 & 5.357602000 & 9.486720000 & 6.544419000 \\ 6 & 4.694823000 & 7.835473000 & 5.314666000 \\ 1 & 4.850318000 & 8.366399000 & 4.373123000 \\ 6 & 2.119941000 & 4.189558000 & 7.858259000 \\ 6 & 1.909989000 & 3.180910000 & 8.806322000 \\ 1 & 2.591881000 & 2.334227000 & 8.849115000 \\ 6 & 0.849388000 & 3.273264000 & 9.714614000 \\ 1 & 0.705427000 & 2.479937000 & 10.451105000 \\ 6 & -0.014919000 & 4.369303000 & 9.682997000 \\ 1 & -0.841368000 & 4.439965000 & 10.393527000 \\ 6 & 0.187857000 & 5.381309000 & 8.738377000 \\ 1 & -0.478235000 & 6.246420000 & 8.707162000 \\ 6 & 1.247969000 & 5.292117000 & 7.834142000 \\ 1 & 1.402951000 & 6.099731000 & 7.116720000 \\ 6 & 4.212410000 & 6.522180000 & 5.291180000 \\ 6 & 4.011085000 & 6.044660000 & 4.332092000 \\ 6 & 2.212018000 & 0.444071000 & 6.738344000 \\ & 2.684073000 & -0.230861000 & 7.875463000\end{array}$




$\begin{array}{lrrr}1 & 3.755154000 & -0.267211000 & 8.078084000 \\ 6 & 1.793179000 & -0.839846000 & 8.763067000 \\ 1 & 2.181313000 & -1.364316000 & 9.638903000 \\ 6 & 0.414873000 & -0.765770000 & 8.538420000 \\ 1 & -0.282654000 & -1.232425000 & 9.237253000 \\ 6 & -0.064786000 & -0.081836000 & 7.417801000 \\ 1 & -1.139778000 & -0.008077000 & 7.238790000 \\ 6 & 0.826722000 & 0.515722000 & 6.521153000 \\ 1 & 0.433997000 & 1.046287000 & 5.652700000 \\ 6 & 3.665268000 & -0.161887000 & 4.340178000 \\ 6 & 4.550025000 & 0.010702000 & 3.263192000 \\ 1 & 5.132490000 & 0.927671000 & 3.180744000 \\ 6 & 4.712363000 & -0.987510000 & 2.299858000 \\ 1 & 5.406643000 & -0.829207000 & 1.471861000 \\ 6 & 3.998931000 & -2.186284000 & 2.403074000 \\ 1 & 4.128833000 & -2.970460000 & 1.654333000 \\ 6 & 3.119895000 & -2.372229000 & 3.473273000 \\ 1 & 2.556718000 & -3.303735000 & 3.564902000 \\ 6 & 2.952109000 & -1.368495000 & 4.433659000 \\ 1 & 2.262734000 & -1.530670000 & 5.262916000\end{array}$

Energy $=-5400.697242$ A.U. 


\section{X-Ray Crystallographic Data}

$3.1{ }^{\text {tBu }}$ dppe

REFERENCE NUMBER: neijk01

CRYSTAL STRUCTURE REPORT

$$
\mathrm{C}_{58} \mathrm{H}_{88} \mathrm{P}_{2}
$$

Report prepared for:

J. Kneebone, Prof. M. Neidig

November 30, 2012

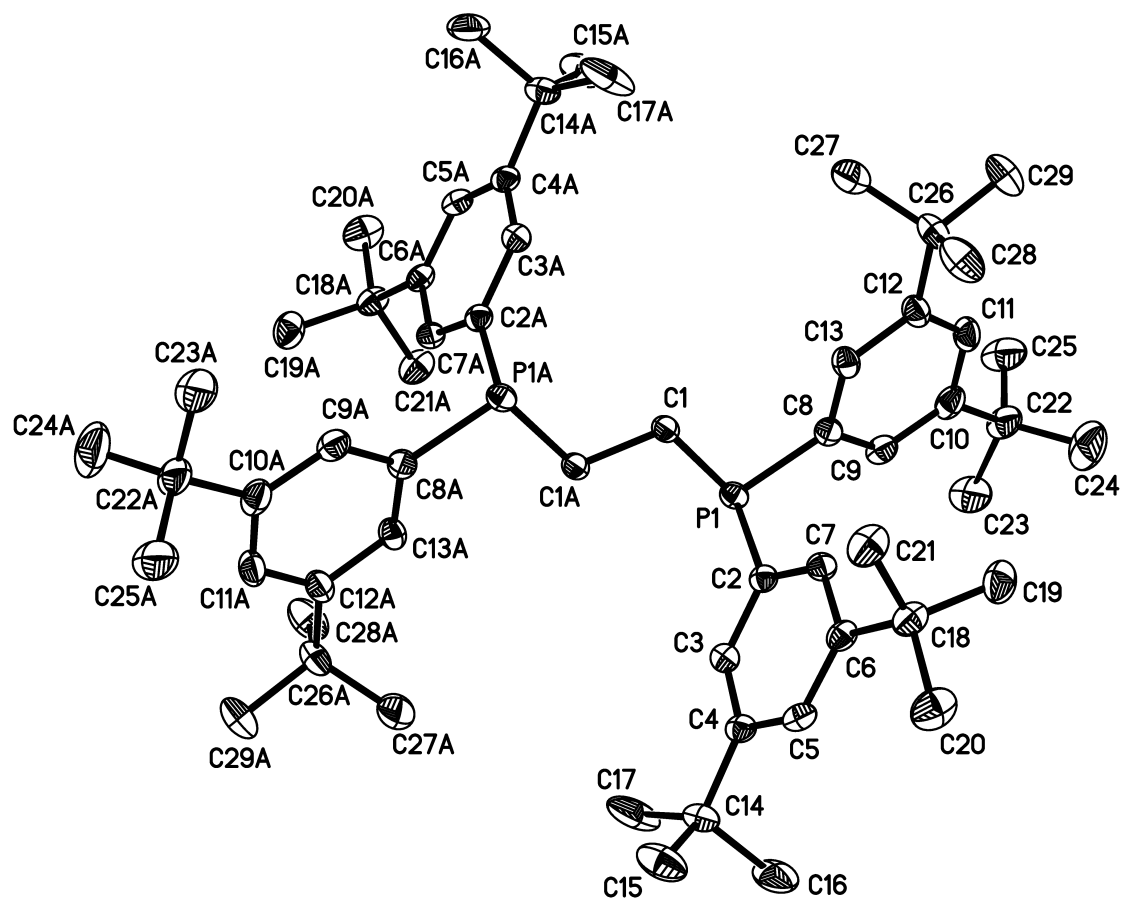

William W. Brennessel

X-ray Crystallographic Facility

Department of Chemistry, University of Rochester

120 Trustee Road

Rochester, NY 14627 


\section{Data collection}

A crystal $\left(0.38 \times 0.30 \times 0.12 \mathrm{~mm}^{3}\right)$ was placed onto the tip of a $0.1 \mathrm{~mm}$ diameter glass capillary tube or fiber and mounted on a Bruker SMART APEX II CCD Platform diffractometer for a data collection at 100.0(5) K. ${ }^{1}$ A preliminary set of cell constants and an orientation matrix were calculated from reflections harvested from three orthogonal wedges of reciprocal space. The full data collection was carried out using MoK $\alpha$ radiation (graphite monochromator) with a frame time of 45 seconds and a detector distance of $4.03 \mathrm{~cm}$. A randomly oriented region of reciprocal space was surveyed: five major sections of frames were collected with $0.50^{\circ}$ steps in $\omega$ at five different $\phi$

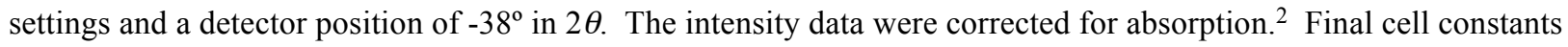
were calculated from the xyz centroids of 3989 strong reflections from the actual data collection after integration. ${ }^{3}$ See Table 1 for additional crystal and refinement information.

\section{Structure solution and refinement}

The structure was solved using SIR9 $7^{4}$ and refined using SHELXL-2012. ${ }^{5}$ The space group $P 2{ }_{1} / c$ was determined based on systematic absences. A direct-methods solution was calculated which provided most non-hydrogen atoms from the E-map. Full-matrix least squares / difference Fourier cycles were performed which located the remaining non-hydrogen atoms. All non-hydrogen atoms were refined with anisotropic displacement parameters. All hydrogen atoms were placed in ideal positions and refined as riding atoms with relative isotropic displacement parameters. The final full matrix least squares refinement converged to $R 1=0.0512\left(F^{2}, I>2 \sigma(I)\right)$ and $w R 2=$ $0.1270\left(F^{2}\right.$, all data).

\section{Structure description}

The structure is the one suggested. The molecule lies in a crystallographic inversion center; thus, one-half is unique. Four (two unique) tert-butyl groups are modeled as rotationally disordered over two positions each (70:30 and 59:41, for groups originating at atoms C14 and C22, respectively).

Unless noted otherwise all structural diagrams containing thermal displacement ellipsoids are drawn at the $50 \%$ probability level.

Data collection, structure solution, and structure refinement were conducted at the X-ray Crystallographic Facility, B51 Hutchison Hall, Department of Chemistry, University of Rochester. All publications arising from this report MUST either 1) include William W. Brennessel as a coauthor or 2) acknowledge William W. Brennessel and the Xray Crystallographic Facility of the Department of Chemistry at the University of Rochester. 
1 APEX2, version 2012.4-3; Bruker AXS: Madison, WI, 2012.

2 Sheldrick, G. M. SADABS, version 2008/1; University of Göttingen: Göttingen, Germany, 2008.

3 SAINT, version 7.68A; Bruker AXS: Madison, WI, 2009.

4 Altomare, A.; Burla, M. C.; Camalli, M.; Cascarano, G. L.; Giacovazzo, C.; Guagliardi, A.; Moliterni, A. G. G.;

Polidori, G.; Spagna, R. SIR97: A new program for solving and refining crystal structures; Istituto di Cristallografia, CNR: Bari, Italy, 1999.

5 Sheldrick, G. M. SHELXL-2012 University of Göttingen: Göttingen, Germany, 2012.

Some equations of interest:

$$
\begin{gathered}
R_{\mathrm{int}}=\Sigma\left|F_{\mathrm{o}}{ }^{2}-<F_{\mathrm{o}}{ }^{2}>\right| / \Sigma\left|F_{\mathrm{o}}{ }^{2}\right| \\
R 1=\Sigma|| F_{\mathrm{o}}|-| F_{\mathrm{c}} \| / \Sigma\left|F_{\mathrm{o}}\right| \\
w R 2=\left[\Sigma\left[w\left(F_{\mathrm{o}}{ }^{2}-F_{\mathrm{c}}{ }^{2}\right)^{2}\right] / \Sigma\left[w\left(F_{\mathrm{o}}{ }^{2}\right)^{2}\right]\right]^{1 / 2} \\
\text { where } w=1 /\left[\sigma^{2}\left(F_{\mathrm{o}}{ }^{2}\right)+(a P)^{2}+b P\right] \text { and } \\
P=1 / 3 \max \left(0, F_{\mathrm{o}}{ }^{2}\right)+2 / 3 F_{\mathrm{c}}{ }^{2} \\
\text { GOF }=S=\left[\Sigma\left[w\left(F_{\mathrm{o}}{ }^{2}-F_{\mathrm{c}}{ }^{2}\right)^{2}\right] /(m-n)\right]^{1 / 2}
\end{gathered}
$$

where $m=$ number of reflections and $n=$ number of parameters

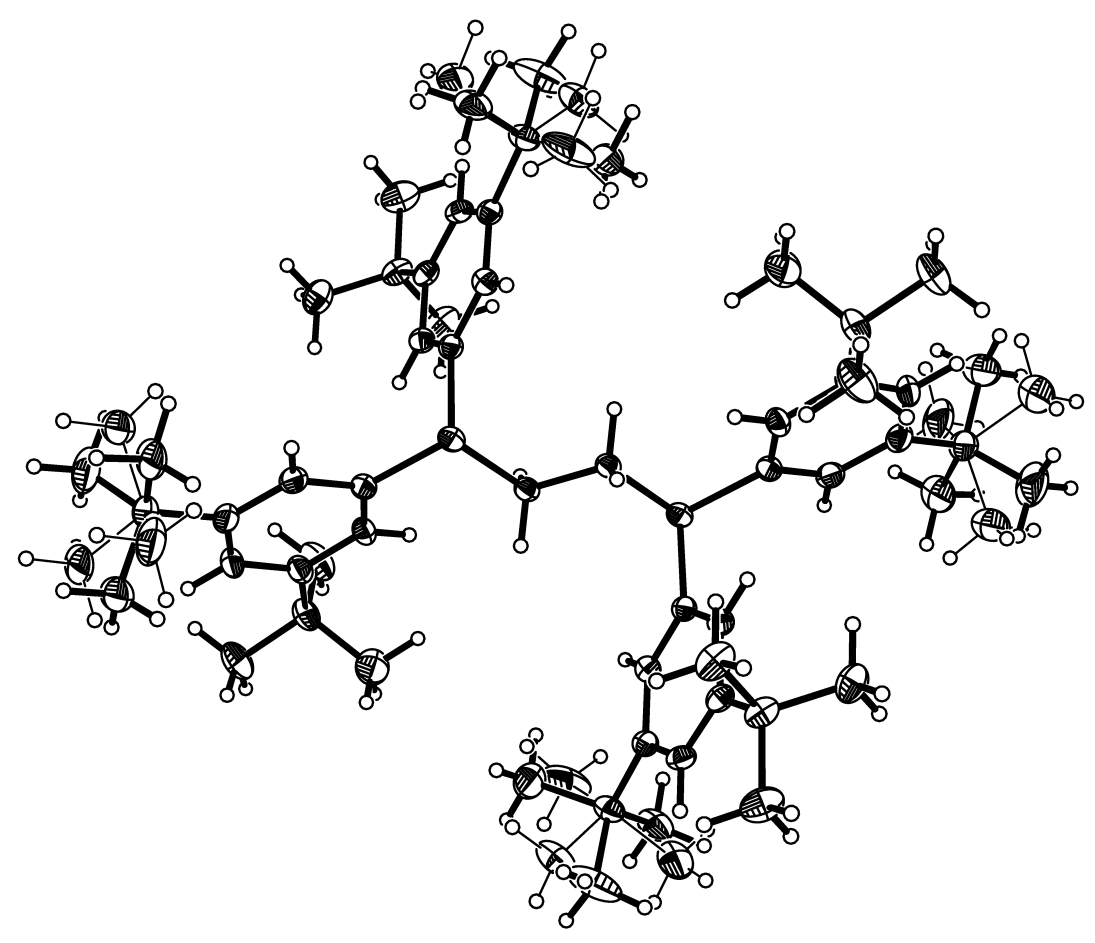




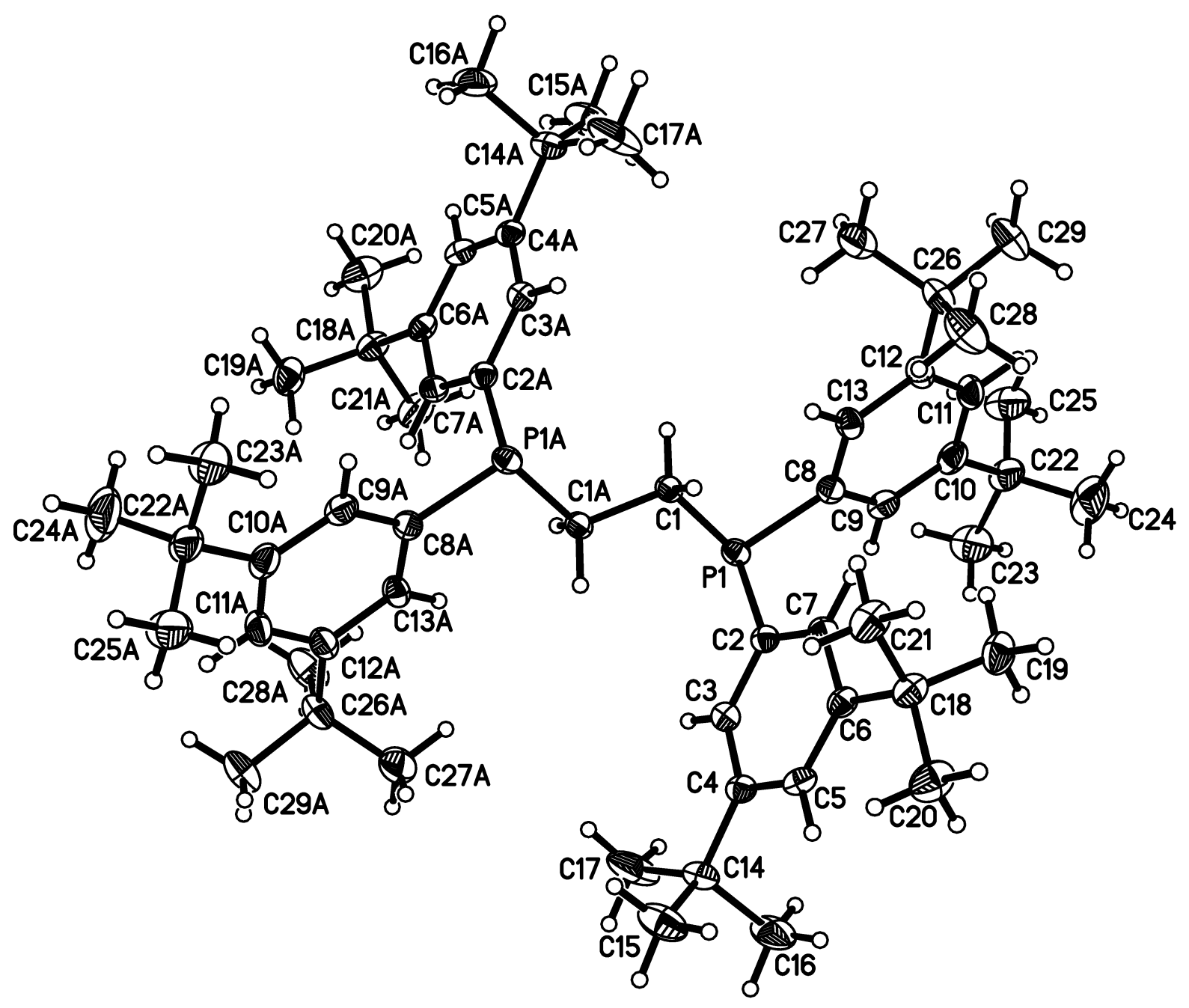


Table S1. Crystal data and structure refinement for neijk01.

\begin{tabular}{|c|c|c|}
\hline Identification code & \multicolumn{2}{|l|}{ neijk01 } \\
\hline Empirical formula & \multicolumn{2}{|l|}{ C58 H88 P2 } \\
\hline Formula weight & \multicolumn{2}{|l|}{847.22} \\
\hline Temperature & \multicolumn{2}{|l|}{$100.0(5) \mathrm{K}$} \\
\hline Wavelength & \multicolumn{2}{|l|}{$0.71073 \AA$} \\
\hline Crystal system & \multicolumn{2}{|l|}{ monoclinic } \\
\hline Space group & \multicolumn{2}{|l|}{$P 2_{1} / c$} \\
\hline \multirow[t]{3}{*}{ Unit cell dimensions } & $a=14.6204(15) \AA$ & $\alpha=90^{\circ}$ \\
\hline & $b=18.6371(19) \AA$ & $\beta=97.648(2)^{\circ}$ \\
\hline & $c=10.1420(10) \AA$ & $\gamma=90^{\circ}$ \\
\hline Volume & \multicolumn{2}{|l|}{$2738.9(5) \AA^{3}$} \\
\hline$Z$ & \multicolumn{2}{|l|}{2} \\
\hline Density (calculated) & \multicolumn{2}{|l|}{$1.027 \mathrm{Mg} / \mathrm{m}^{3}$} \\
\hline Absorption coefficient & \multicolumn{2}{|l|}{$0.113 \mathrm{~mm}^{-1}$} \\
\hline$F(000)$ & \multicolumn{2}{|l|}{932} \\
\hline Crystal color, morphology & \multicolumn{2}{|l|}{ colorless, plate } \\
\hline Crystal size & \multicolumn{2}{|c|}{$0.38 \times 0.30 \times 0.12 \mathrm{~mm}^{3}$} \\
\hline Theta range for data collection & \multicolumn{2}{|l|}{1.780 to $30.508^{\circ}$} \\
\hline Index ranges & \multicolumn{2}{|c|}{$-20 \leq h \leq 20,-26 \leq k \leq 26,-14 \leq l \leq 14$} \\
\hline Reflections collected & \multicolumn{2}{|l|}{50173} \\
\hline Independent reflections & \multicolumn{2}{|c|}{$8357[R($ int $)=0.0953]$} \\
\hline Observed reflections & \multicolumn{2}{|l|}{5438} \\
\hline Completeness to theta $=30.510^{\circ}$ & \multicolumn{2}{|l|}{$99.9 \%$} \\
\hline Absorption correction & \multicolumn{2}{|l|}{ Multi-scan } \\
\hline Max. and min. transmission & \multicolumn{2}{|l|}{0.7461 and 0.6420} \\
\hline Refinement method & \multicolumn{2}{|c|}{ Full-matrix least-squares on $F^{2}$} \\
\hline Data / restraints / parameters & \multicolumn{2}{|l|}{8357 / 18 / 345} \\
\hline Goodness-of-fit on $F^{2}$ & \multicolumn{2}{|l|}{1.023} \\
\hline Final $R$ indices $[I>2 \operatorname{sigma}(I)]$ & \multicolumn{2}{|c|}{$R 1=0.0512, w R 2=0.1080$} \\
\hline$R$ indices (all data) & \multicolumn{2}{|c|}{$R 1=0.0943, w R 2=0.1270$} \\
\hline Extinction coefficient & \multicolumn{2}{|l|}{$\mathrm{n} / \mathrm{a}$} \\
\hline Largest diff. peak and hole & \multicolumn{2}{|c|}{0.344 and -0.301 e. $\AA^{-3}$} \\
\hline
\end{tabular}


Table S2. Atomic coordinates $\left(\mathrm{x} 10^{4}\right)$ and equivalent isotropic displacement parameters $\left(\AA^{2} \times 10^{3}\right)$ for neijk01. $U_{e q}$ is defined as one third of the trace of the orthogonalized $U_{i j}$ tensor.

\begin{tabular}{|c|c|c|c|c|}
\hline & $\mathrm{x}$ & $\mathrm{y}$ & $\mathrm{z}$ & $\mathrm{U}_{\mathrm{eq}}$ \\
\hline P1 & $9308(1)$ & $289(1)$ & 2971(1) & $20(1)$ \\
\hline $\mathrm{C} 1$ & $9527(1)$ & $-115(1)$ & 4649(1) & $19(1)$ \\
\hline $\mathrm{C} 2$ & $8916(1)$ & $1178(1)$ & $3424(1)$ & $19(1)$ \\
\hline $\mathrm{C} 3$ & $9524(1)$ & 1751(1) & 3371(1) & $20(1)$ \\
\hline $\mathrm{C} 4$ & $9299(1)$ & $2438(1)$ & $3749(1)$ & $20(1)$ \\
\hline $\mathrm{C} 5$ & $8442(1)$ & $2537(1)$ & 4179(1) & $22(1)$ \\
\hline C6 & $7815(1)$ & 1983(1) & 4242(1) & 21(1) \\
\hline $\mathrm{C} 7$ & $8066(1)$ & 1301(1) & 3851(1) & $20(1)$ \\
\hline $\mathrm{C} 8$ & $8228(1)$ & $-155(1)$ & 2303(1) & 21(1) \\
\hline C9 & 7978(1) & $-141(1)$ & $924(2)$ & $25(1)$ \\
\hline $\mathrm{C} 10$ & 7201(1) & $-502(1)$ & $325(2)$ & $28(1)$ \\
\hline $\mathrm{C} 11$ & $6680(1)$ & $-889(1)$ & $1134(2)$ & $28(1)$ \\
\hline $\mathrm{C} 12$ & $6909(1)$ & $-918(1)$ & $2510(2)$ & $24(1)$ \\
\hline $\mathrm{C} 13$ & $7686(1)$ & $-541(1)$ & $3072(2)$ & $22(1)$ \\
\hline $\mathrm{C} 14$ & $9945(1)$ & $3075(1)$ & $3638(2)$ & $26(1)$ \\
\hline $\mathrm{C} 15$ & $10042(3)$ & $3527(2)$ & 4887(4) & $42(1)$ \\
\hline $\mathrm{C} 16$ & $9497(3)$ & $3547(2)$ & 2463(3) & $50(1)$ \\
\hline $\mathrm{C} 17$ & $10902(2)$ & $2860(2)$ & $3345(5)$ & $53(1)$ \\
\hline C15' & $9679(9)$ & $3745(5)$ & $4398(12)$ & $56(3)$ \\
\hline C16' & 9989(7) & $3245(4)$ & $2242(6)$ & $39(2)$ \\
\hline $\mathrm{C} 17^{\prime}$ & $10925(6)$ & $2850(4)$ & $4328(13)$ & $58(3)$ \\
\hline $\mathrm{C} 18$ & $6880(1)$ & $2084(1)$ & $4745(2)$ & $26(1)$ \\
\hline C19 & $6106(1)$ & $1806(1)$ & 3701(2) & $33(1)$ \\
\hline $\mathrm{C} 20$ & $6682(1)$ & $2870(1)$ & $5022(2)$ & $37(1)$ \\
\hline $\mathrm{C} 21$ & 6881(1) & $1655(1)$ & $6035(2)$ & $33(1)$ \\
\hline $\mathrm{C} 22$ & $6925(1)$ & $-488(1)$ & $-1191(2)$ & $36(1)$ \\
\hline $\mathrm{C} 23$ & $7662(3)$ & $-59(2)$ & $-1932(3)$ & $49(1)$ \\
\hline $\mathrm{C} 24$ & 6023(3) & $-111(2)$ & $-1557(4)$ & $57(1)$ \\
\hline $\mathrm{C} 25$ & 6924(3) & $-1242(2)$ & $-1735(3)$ & $51(1)$ \\
\hline C23' & $6007(4)$ & $-972(3)$ & $-1607(4)$ & $46(2)$ \\
\hline C24' & $7663(4)$ & $-779(4)$ & $-1861(4)$ & $54(2)$ \\
\hline
\end{tabular}




\begin{tabular}{lrrrr}
$\mathrm{C} 25^{\prime}$ & $6638(4)$ & $264(3)$ & $-1551(5)$ & $44(1)$ \\
$\mathrm{C} 26$ & $6345(1)$ & $-1336(1)$ & $3421(2)$ & $30(1)$ \\
$\mathrm{C} 27$ & $6982(1)$ & $-1824(1)$ & $4348(2)$ & $44(1)$ \\
$\mathrm{C} 28$ & $5869(1)$ & $-797(1)$ & $4248(2)$ & $42(1)$ \\
$\mathrm{C} 29$ & $5607(1)$ & $-1809(1)$ & $2643(2)$ & $40(1)$ \\
\hline
\end{tabular}


Table S3. Bond lengths $[\AA]$ and angles $\left[{ }^{\circ}\right]$ for neijk01.

\begin{tabular}{|c|c|c|c|}
\hline $\mathrm{P}(1)-\mathrm{C}(8)$ & $1.8308(15)$ & $\mathrm{C}(15)-\mathrm{H}(15 \mathrm{~A})$ & 0.9800 \\
\hline$P(1)-C(2)$ & $1.8327(15)$ & $\mathrm{C}(15)-\mathrm{H}(15 \mathrm{~B})$ & 0.9800 \\
\hline $\mathrm{P}(1)-\mathrm{C}(1)$ & $1.8491(15)$ & $\mathrm{C}(15)-\mathrm{H}(15 \mathrm{C})$ & 0.9800 \\
\hline$C(1)-C(1) \# 1$ & $1.529(3)$ & $\mathrm{C}(16)-\mathrm{H}(16 \mathrm{~A})$ & 0.9800 \\
\hline $\mathrm{C}(1)-\mathrm{H}(1 \mathrm{~A})$ & 0.9900 & $\mathrm{C}(16)-\mathrm{H}(16 \mathrm{~B})$ & 0.9800 \\
\hline $\mathrm{C}(1)-\mathrm{H}(1 \mathrm{~B})$ & 0.9900 & $\mathrm{C}(16)-\mathrm{H}(16 \mathrm{C})$ & 0.9800 \\
\hline$C(2)-C(7)$ & $1.3878(19)$ & $\mathrm{C}(17)-\mathrm{H}(17 \mathrm{~A})$ & 0.9800 \\
\hline$C(2)-C(3)$ & $1.395(2)$ & $\mathrm{C}(17)-\mathrm{H}(17 \mathrm{~B})$ & 0.9800 \\
\hline $\mathrm{C}(3)-\mathrm{C}(4)$ & $1.390(2)$ & $\mathrm{C}(17)-\mathrm{H}(17 \mathrm{C})$ & 0.9800 \\
\hline $\mathrm{C}(3)-\mathrm{H}(3)$ & 0.9500 & $\mathrm{C}\left(15^{\prime}\right)-\mathrm{H}(15 \mathrm{D})$ & 0.9800 \\
\hline$C(4)-C(5)$ & $1.392(2)$ & $\mathrm{C}\left(15^{\prime}\right)-\mathrm{H}(15 \mathrm{E})$ & 0.9800 \\
\hline $\mathrm{C}(4)-\mathrm{C}(14)$ & $1.529(2)$ & $\mathrm{C}\left(15^{\prime}\right)-\mathrm{H}(15 \mathrm{~F})$ & 0.9800 \\
\hline$C(5)-C(6)$ & $1.389(2)$ & $C\left(16^{\prime}\right)-H(16 D)$ & 0.9800 \\
\hline $\mathrm{C}(5)-\mathrm{H}(5)$ & 0.9500 & $\mathrm{C}\left(16^{\prime}\right)-\mathrm{H}(16 \mathrm{E})$ & 0.9800 \\
\hline$C(6)-C(7)$ & $1.395(2)$ & $\mathrm{C}\left(16^{\prime}\right)-\mathrm{H}(16 \mathrm{~F})$ & 0.9800 \\
\hline$C(6)-C(18)$ & $1.533(2)$ & $\mathrm{C}\left(17^{\prime}\right)-\mathrm{H}(17 \mathrm{D})$ & 0.9800 \\
\hline $\mathrm{C}(7)-\mathrm{H}(7)$ & 0.9500 & $\mathrm{C}\left(17^{\prime}\right)-\mathrm{H}(17 \mathrm{E})$ & 0.9800 \\
\hline $\mathrm{C}(8)-\mathrm{C}(13)$ & $1.385(2)$ & $\mathrm{C}\left(17^{\prime}\right)-\mathrm{H}(17 \mathrm{~F})$ & 0.9800 \\
\hline $\mathrm{C}(8)-\mathrm{C}(9)$ & $1.398(2)$ & $C(18)-C(20)$ & $1.526(2)$ \\
\hline$C(9)-C(10)$ & $1.388(2)$ & $\mathrm{C}(18)-\mathrm{C}(19)$ & $1.533(2)$ \\
\hline $\mathrm{C}(9)-\mathrm{H}(9)$ & 0.9500 & $C(18)-C(21)$ & $1.533(2)$ \\
\hline$C(10)-C(11)$ & $1.393(2)$ & $\mathrm{C}(19)-\mathrm{H}(19 \mathrm{~A})$ & 0.9800 \\
\hline$C(10)-C(22)$ & $1.537(2)$ & $\mathrm{C}(19)-\mathrm{H}(19 \mathrm{~B})$ & 0.9800 \\
\hline $\mathrm{C}(11)-\mathrm{C}(12)$ & $1.392(2)$ & $\mathrm{C}(19)-\mathrm{H}(19 \mathrm{C})$ & 0.9800 \\
\hline $\mathrm{C}(11)-\mathrm{H}(11)$ & 0.9500 & $\mathrm{C}(20)-\mathrm{H}(20 \mathrm{~A})$ & 0.9800 \\
\hline $\mathrm{C}(12)-\mathrm{C}(13)$ & $1.392(2)$ & $\mathrm{C}(20)-\mathrm{H}(20 \mathrm{~B})$ & 0.9800 \\
\hline$C(12)-C(26)$ & $1.531(2)$ & $\mathrm{C}(20)-\mathrm{H}(20 \mathrm{C})$ & 0.9800 \\
\hline $\mathrm{C}(13)-\mathrm{H}(13)$ & 0.9500 & $\mathrm{C}(21)-\mathrm{H}(21 \mathrm{~A})$ & 0.9800 \\
\hline$C(14)-C\left(16^{\prime}\right)$ & $1.461(6)$ & $\mathrm{C}(21)-\mathrm{H}(21 \mathrm{~B})$ & 0.9800 \\
\hline$C(14)-C(15)$ & $1.513(4)$ & $\mathrm{C}(21)-\mathrm{H}(21 \mathrm{C})$ & 0.9800 \\
\hline$C(14)-C(17)$ & $1.522(3)$ & $C(22)-C\left(24^{\prime}\right)$ & $1.455(5)$ \\
\hline$C(14)-C\left(15^{\prime}\right)$ & $1.544(9)$ & $C(22)-C\left(25^{\prime}\right)$ & $1.494(5)$ \\
\hline$C(14)-C(16)$ & $1.554(4)$ & $C(22)-C(24)$ & $1.496(4)$ \\
\hline $\mathrm{C}(14)-\mathrm{C}\left(17^{\prime}\right)$ & $1.566(7)$ & $C(22)-C(25)$ & $1.511(4)$ \\
\hline
\end{tabular}




\begin{tabular}{|c|c|c|c|}
\hline$C(22)-C(23)$ & $1.607(4)$ & $\mathrm{C}(1) \# 1-\mathrm{C}(1)-\mathrm{H}(1 \mathrm{~A})$ & 109.6 \\
\hline $\mathrm{C}(22)-\mathrm{C}\left(23^{\prime}\right)$ & $1.625(5)$ & $\mathrm{P}(1)-\mathrm{C}(1)-\mathrm{H}(1 \mathrm{~A})$ & 109.6 \\
\hline $\mathrm{C}(23)-\mathrm{H}(23 \mathrm{~A})$ & 0.9800 & $\mathrm{C}(1) \# 1-\mathrm{C}(1)-\mathrm{H}(1 \mathrm{~B})$ & 109.6 \\
\hline $\mathrm{C}(23)-\mathrm{H}(23 \mathrm{~B})$ & 0.9800 & $\mathrm{P}(1)-\mathrm{C}(1)-\mathrm{H}(1 \mathrm{~B})$ & 109.6 \\
\hline $\mathrm{C}(23)-\mathrm{H}(23 \mathrm{C})$ & 0.9800 & $\mathrm{H}(1 \mathrm{~A})-\mathrm{C}(1)-\mathrm{H}(1 \mathrm{~B})$ & 108.1 \\
\hline $\mathrm{C}(24)-\mathrm{H}(24 \mathrm{~A})$ & 0.9800 & $C(7)-C(2)-C(3)$ & $119.25(13)$ \\
\hline $\mathrm{C}(24)-\mathrm{H}(24 \mathrm{~B})$ & 0.9800 & $C(7)-C(2)-P(1)$ & $123.39(11)$ \\
\hline $\mathrm{C}(24)-\mathrm{H}(24 \mathrm{C})$ & 0.9800 & $\mathrm{C}(3)-\mathrm{C}(2)-\mathrm{P}(1)$ & $117.33(11)$ \\
\hline $\mathrm{C}(25)-\mathrm{H}(25 \mathrm{~A})$ & 0.9800 & $\mathrm{C}(4)-\mathrm{C}(3)-\mathrm{C}(2)$ & $121.26(13)$ \\
\hline $\mathrm{C}(25)-\mathrm{H}(25 \mathrm{~B})$ & 0.9800 & $\mathrm{C}(4)-\mathrm{C}(3)-\mathrm{H}(3)$ & 119.4 \\
\hline $\mathrm{C}(25)-\mathrm{H}(25 \mathrm{C})$ & 0.9800 & $\mathrm{C}(2)-\mathrm{C}(3)-\mathrm{H}(3)$ & 119.4 \\
\hline $\mathrm{C}\left(23^{\prime}\right)-\mathrm{H}(23 \mathrm{D})$ & 0.9800 & $C(3)-C(4)-C(5)$ & $117.65(13)$ \\
\hline $\mathrm{C}\left(23^{\prime}\right)-\mathrm{H}(23 \mathrm{E})$ & 0.9800 & $\mathrm{C}(3)-\mathrm{C}(4)-\mathrm{C}(14)$ & $121.60(13)$ \\
\hline $\mathrm{C}\left(23^{\prime}\right)-\mathrm{H}(23 \mathrm{~F})$ & 0.9800 & $\mathrm{C}(5)-\mathrm{C}(4)-\mathrm{C}(14)$ & $120.70(13)$ \\
\hline $\mathrm{C}\left(24^{\prime}\right)-\mathrm{H}(24 \mathrm{D})$ & 0.9800 & $C(6)-C(5)-C(4)$ & $122.93(14)$ \\
\hline $\mathrm{C}\left(24^{\prime}\right)-\mathrm{H}(24 \mathrm{E})$ & 0.9800 & $\mathrm{C}(6)-\mathrm{C}(5)-\mathrm{H}(5)$ & 118.5 \\
\hline $\mathrm{C}\left(24^{\prime}\right)-\mathrm{H}(24 \mathrm{~F})$ & 0.9800 & $\mathrm{C}(4)-\mathrm{C}(5)-\mathrm{H}(5)$ & 118.5 \\
\hline $\mathrm{C}\left(25^{\prime}\right)-\mathrm{H}(25 \mathrm{D})$ & 0.9800 & $\mathrm{C}(5)-\mathrm{C}(6)-\mathrm{C}(7)$ & $117.67(13)$ \\
\hline $\mathrm{C}\left(25^{\prime}\right)-\mathrm{H}(25 \mathrm{E})$ & 0.9800 & $\mathrm{C}(5)-\mathrm{C}(6)-\mathrm{C}(18)$ & $123.17(13)$ \\
\hline $\mathrm{C}\left(25^{\prime}\right)-\mathrm{H}(25 \mathrm{~F})$ & 0.9800 & $\mathrm{C}(7)-\mathrm{C}(6)-\mathrm{C}(18)$ & $119.15(13)$ \\
\hline$C(26)-C(29)$ & $1.529(2)$ & $C(2)-C(7)-C(6)$ & $121.25(13)$ \\
\hline$C(26)-C(27)$ & $1.532(3)$ & $\mathrm{C}(2)-\mathrm{C}(7)-\mathrm{H}(7)$ & 119.4 \\
\hline$C(26)-C(28)$ & $1.534(2)$ & $\mathrm{C}(6)-\mathrm{C}(7)-\mathrm{H}(7)$ & 119.4 \\
\hline $\mathrm{C}(27)-\mathrm{H}(27 \mathrm{~A})$ & 0.9800 & $\mathrm{C}(13)-\mathrm{C}(8)-\mathrm{C}(9)$ & $118.63(14)$ \\
\hline $\mathrm{C}(27)-\mathrm{H}(27 \mathrm{~B})$ & 0.9800 & $C(13)-C(8)-P(1)$ & $123.81(11)$ \\
\hline $\mathrm{C}(27)-\mathrm{H}(27 \mathrm{C})$ & 0.9800 & $\mathrm{C}(9)-\mathrm{C}(8)-\mathrm{P}(1)$ & $117.41(12)$ \\
\hline $\mathrm{C}(28)-\mathrm{H}(28 \mathrm{~A})$ & 0.9800 & $\mathrm{C}(10)-\mathrm{C}(9)-\mathrm{C}(8)$ & $121.28(15)$ \\
\hline $\mathrm{C}(28)-\mathrm{H}(28 \mathrm{~B})$ & 0.9800 & $\mathrm{C}(10)-\mathrm{C}(9)-\mathrm{H}(9)$ & 119.4 \\
\hline $\mathrm{C}(28)-\mathrm{H}(28 \mathrm{C})$ & 0.9800 & $\mathrm{C}(8)-\mathrm{C}(9)-\mathrm{H}(9)$ & 119.4 \\
\hline $\mathrm{C}(29)-\mathrm{H}(29 \mathrm{~A})$ & 0.9800 & $\mathrm{C}(9)-\mathrm{C}(10)-\mathrm{C}(11)$ & $118.22(14)$ \\
\hline C(29)-H(29B) & 0.9800 & $\mathrm{C}(9)-\mathrm{C}(10)-\mathrm{C}(22)$ & $121.32(16)$ \\
\hline $\mathrm{C}(29)-\mathrm{H}(29 \mathrm{C})$ & 0.9800 & $\mathrm{C}(11)-\mathrm{C}(10)-\mathrm{C}(22)$ & $120.46(15)$ \\
\hline $\mathrm{C}(8)-\mathrm{P}(1)-\mathrm{C}(2)$ & $102.59(6)$ & $C(12)-C(11)-C(10)$ & $122.20(14)$ \\
\hline $\mathrm{C}(8)-\mathrm{P}(1)-\mathrm{C}(1)$ & $101.06(7)$ & $\mathrm{C}(12)-\mathrm{C}(11)-\mathrm{H}(11)$ & 118.9 \\
\hline$C(2)-P(1)-C(1)$ & $99.14(6)$ & $\mathrm{C}(10)-\mathrm{C}(11)-\mathrm{H}(11)$ & 118.9 \\
\hline $\mathrm{C}(1) \# 1-\mathrm{C}(1)-\mathrm{P}(1)$ & $110.48(13)$ & $\mathrm{C}(11)-\mathrm{C}(12)-\mathrm{C}(13)$ & $117.74(15)$ \\
\hline
\end{tabular}




\begin{tabular}{|c|c|}
\hline $\mathrm{C}(11)-\mathrm{C}(12)-\mathrm{C}(26)$ & $123.22(14)$ \\
\hline $\mathrm{C}(13)-\mathrm{C}(12)-\mathrm{C}(26)$ & $119.03(13)$ \\
\hline$C(8)-C(13)-C(12)$ & $121.93(14)$ \\
\hline $\mathrm{C}(8)-\mathrm{C}(13)-\mathrm{H}(13)$ & 119.0 \\
\hline $\mathrm{C}(12)-\mathrm{C}(13)-\mathrm{H}(13)$ & 119.0 \\
\hline$C(15)-C(14)-C(17)$ & $108.9(2)$ \\
\hline$C\left(16^{\prime}\right)-C(14)-C(4)$ & $110.3(3)$ \\
\hline$C(15)-C(14)-C(4)$ & $111.15(19)$ \\
\hline$C(17)-C(14)-C(4)$ & $113.75(16)$ \\
\hline $\mathrm{C}\left(16^{\prime}\right)-\mathrm{C}(14)-\mathrm{C}\left(15^{\prime}\right)$ & $110.8(5)$ \\
\hline$C(4)-C(14)-C\left(15^{\prime}\right)$ & 113.1(4) \\
\hline $\mathrm{C}(15)-\mathrm{C}(14)-\mathrm{C}(16)$ & $108.0(2)$ \\
\hline $\mathrm{C}(17)-\mathrm{C}(14)-\mathrm{C}(16)$ & $107.7(2)$ \\
\hline$C(4)-C(14)-C(16)$ & $107.15(16)$ \\
\hline $\mathrm{C}\left(16^{\prime}\right)-\mathrm{C}(14)-\mathrm{C}\left(17^{\prime}\right)$ & $109.6(5)$ \\
\hline$C(4)-C(14)-C\left(17^{\prime}\right)$ & $107.2(3)$ \\
\hline $\mathrm{C}\left(15^{\prime}\right)-\mathrm{C}(14)-\mathrm{C}\left(17^{\prime}\right)$ & $105.7(6)$ \\
\hline $\mathrm{C}(14)-\mathrm{C}(15)-\mathrm{H}(15 \mathrm{~A})$ & 109.5 \\
\hline $\mathrm{C}(14)-\mathrm{C}(15)-\mathrm{H}(15 \mathrm{~B})$ & 109.5 \\
\hline $\mathrm{H}(15 \mathrm{~A})-\mathrm{C}(15)-\mathrm{H}(15 \mathrm{~B})$ & 109.5 \\
\hline $\mathrm{C}(14)-\mathrm{C}(15)-\mathrm{H}(15 \mathrm{C})$ & 109.5 \\
\hline $\mathrm{H}(15 \mathrm{~A})-\mathrm{C}(15)-\mathrm{H}(15 \mathrm{C})$ & 109.5 \\
\hline $\mathrm{H}(15 \mathrm{~B})-\mathrm{C}(15)-\mathrm{H}(15 \mathrm{C})$ & 109.5 \\
\hline $\mathrm{C}(14)-\mathrm{C}(16)-\mathrm{H}(16 \mathrm{~A})$ & 109.5 \\
\hline $\mathrm{C}(14)-\mathrm{C}(16)-\mathrm{H}(16 \mathrm{~B})$ & 109.5 \\
\hline $\mathrm{H}(16 \mathrm{~A})-\mathrm{C}(16)-\mathrm{H}(16 \mathrm{~B})$ & 109.5 \\
\hline $\mathrm{C}(14)-\mathrm{C}(16)-\mathrm{H}(16 \mathrm{C})$ & 109.5 \\
\hline $\mathrm{H}(16 \mathrm{~A})-\mathrm{C}(16)-\mathrm{H}(16 \mathrm{C})$ & 109.5 \\
\hline $\mathrm{H}(16 \mathrm{~B})-\mathrm{C}(16)-\mathrm{H}(16 \mathrm{C})$ & 109.5 \\
\hline $\mathrm{C}(14)-\mathrm{C}(17)-\mathrm{H}(17 \mathrm{~A})$ & 109.5 \\
\hline $\mathrm{C}(14)-\mathrm{C}(17)-\mathrm{H}(17 \mathrm{~B})$ & 109.5 \\
\hline $\mathrm{H}(17 \mathrm{~A})-\mathrm{C}(17)-\mathrm{H}(17 \mathrm{~B})$ & 109.5 \\
\hline $\mathrm{C}(14)-\mathrm{C}(17)-\mathrm{H}(17 \mathrm{C})$ & 109.5 \\
\hline $\mathrm{H}(17 \mathrm{~A})-\mathrm{C}(17)-\mathrm{H}(17 \mathrm{C})$ & 109.5 \\
\hline $\mathrm{H}(17 \mathrm{~B})-\mathrm{C}(17)-\mathrm{H}(17 \mathrm{C})$ & 109.5 \\
\hline $\mathrm{C}(14)-\mathrm{C}\left(15^{\prime}\right)-\mathrm{H}(15 \mathrm{D})$ & 109.5 \\
\hline
\end{tabular}

\begin{tabular}{|c|c|}
\hline $\mathrm{C}(14)-\mathrm{C}\left(15^{\prime}\right)-\mathrm{H}(15 \mathrm{E})$ & 109.5 \\
\hline $\mathrm{H}(15 \mathrm{D})-\mathrm{C}\left(15^{\prime}\right)-\mathrm{H}(15 \mathrm{E})$ & 109.5 \\
\hline $\mathrm{C}(14)-\mathrm{C}\left(15^{\prime}\right)-\mathrm{H}(15 \mathrm{~F})$ & 109.5 \\
\hline $\mathrm{H}(15 \mathrm{D})-\mathrm{C}\left(15^{\prime}\right)-\mathrm{H}(15 \mathrm{~F})$ & 109.5 \\
\hline $\mathrm{H}(15 \mathrm{E})-\mathrm{C}\left(15^{\prime}\right)-\mathrm{H}(15 \mathrm{~F})$ & 109.5 \\
\hline $\mathrm{C}(14)-\mathrm{C}\left(16^{\prime}\right)-\mathrm{H}(16 \mathrm{D})$ & 109.5 \\
\hline $\mathrm{C}(14)-\mathrm{C}\left(16^{\prime}\right)-\mathrm{H}(16 \mathrm{E})$ & 109.5 \\
\hline $\mathrm{H}(16 \mathrm{D})-\mathrm{C}\left(16^{\prime}\right)-\mathrm{H}(16 \mathrm{E})$ & 109.5 \\
\hline $\mathrm{C}(14)-\mathrm{C}\left(16^{\prime}\right)-\mathrm{H}(16 \mathrm{~F})$ & 109.5 \\
\hline $\mathrm{H}(16 \mathrm{D})-\mathrm{C}\left(16^{\prime}\right)-\mathrm{H}(16 \mathrm{~F})$ & 109.5 \\
\hline $\mathrm{H}(16 \mathrm{E})-\mathrm{C}\left(16^{\prime}\right)-\mathrm{H}(16 \mathrm{~F})$ & 109.5 \\
\hline $\mathrm{C}(14)-\mathrm{C}\left(17^{\prime}\right)-\mathrm{H}(17 \mathrm{D})$ & 109.5 \\
\hline $\mathrm{C}(14)-\mathrm{C}\left(17^{\prime}\right)-\mathrm{H}(17 \mathrm{E})$ & 109.5 \\
\hline $\mathrm{H}(17 \mathrm{D})-\mathrm{C}\left(17^{\prime}\right)-\mathrm{H}(17 \mathrm{E})$ & 109.5 \\
\hline $\mathrm{C}(14)-\mathrm{C}\left(17^{\prime}\right)-\mathrm{H}(17 \mathrm{~F})$ & 109.5 \\
\hline $\mathrm{H}(17 \mathrm{D})-\mathrm{C}\left(17^{\prime}\right)-\mathrm{H}(17 \mathrm{~F})$ & 109.5 \\
\hline $\mathrm{H}(17 \mathrm{E})-\mathrm{C}\left(17^{\prime}\right)-\mathrm{H}(17 \mathrm{~F})$ & 109.5 \\
\hline C(20)-C(18)-C(19) & $108.12(14)$ \\
\hline$C(20)-C(18)-C(6)$ & $112.26(13)$ \\
\hline $\mathrm{C}(19)-\mathrm{C}(18)-\mathrm{C}(6)$ & $109.71(13)$ \\
\hline $\mathrm{C}(20)-\mathrm{C}(18)-\mathrm{C}(21)$ & $108.82(14)$ \\
\hline C(19)-C(18)-C(21) & $109.26(14)$ \\
\hline$C(6)-C(18)-C(21)$ & $108.63(12)$ \\
\hline $\mathrm{C}(18)-\mathrm{C}(19)-\mathrm{H}(19 \mathrm{~A})$ & 109.5 \\
\hline C(18)-C(19)-H(19B) & 109.5 \\
\hline $\mathrm{H}(19 \mathrm{~A})-\mathrm{C}(19)-\mathrm{H}(19 \mathrm{~B})$ & 109.5 \\
\hline C(18)-C(19)-H(19C) & 109.5 \\
\hline $\mathrm{H}(19 \mathrm{~A})-\mathrm{C}(19)-\mathrm{H}(19 \mathrm{C})$ & 109.5 \\
\hline H(19B)-C(19)-H(19C) & 109.5 \\
\hline $\mathrm{C}(18)-\mathrm{C}(20)-\mathrm{H}(20 \mathrm{~A})$ & 109.5 \\
\hline $\mathrm{C}(18)-\mathrm{C}(20)-\mathrm{H}(20 \mathrm{~B})$ & 109.5 \\
\hline $\mathrm{H}(20 \mathrm{~A})-\mathrm{C}(20)-\mathrm{H}(20 \mathrm{~B})$ & 109.5 \\
\hline $\mathrm{C}(18)-\mathrm{C}(20)-\mathrm{H}(20 \mathrm{C})$ & 109.5 \\
\hline $\mathrm{H}(20 \mathrm{~A})-\mathrm{C}(20)-\mathrm{H}(20 \mathrm{C})$ & 109.5 \\
\hline $\mathrm{H}(20 \mathrm{~B})-\mathrm{C}(20)-\mathrm{H}(20 \mathrm{C})$ & 109.5 \\
\hline $\mathrm{C}(18)-\mathrm{C}(21)-\mathrm{H}(21 \mathrm{~A})$ & 109.5 \\
\hline
\end{tabular}




\begin{tabular}{|c|c|c|c|}
\hline $\mathrm{C}(18)-\mathrm{C}(21)-\mathrm{H}(21 \mathrm{~B})$ & 109.5 & $\mathrm{C}(22)-\mathrm{C}\left(23^{\prime}\right)-\mathrm{H}(23 \mathrm{E})$ & 109.5 \\
\hline $\mathrm{H}(21 \mathrm{~A})-\mathrm{C}(21)-\mathrm{H}(21 \mathrm{~B})$ & 109.5 & $\mathrm{H}(23 \mathrm{D})-\mathrm{C}\left(23^{\prime}\right)-\mathrm{H}(23 \mathrm{E})$ & 109.5 \\
\hline $\mathrm{C}(18)-\mathrm{C}(21)-\mathrm{H}(21 \mathrm{C})$ & 109.5 & $\mathrm{C}(22)-\mathrm{C}\left(23^{\prime}\right)-\mathrm{H}(23 \mathrm{~F})$ & 109.5 \\
\hline $\mathrm{H}(21 \mathrm{~A})-\mathrm{C}(21)-\mathrm{H}(21 \mathrm{C})$ & 109.5 & $\mathrm{H}(23 \mathrm{D})-\mathrm{C}\left(23^{\prime}\right)-\mathrm{H}(23 \mathrm{~F})$ & 109.5 \\
\hline $\mathrm{H}(21 \mathrm{~B})-\mathrm{C}(21)-\mathrm{H}(21 \mathrm{C})$ & 109.5 & $\mathrm{H}(23 \mathrm{E})-\mathrm{C}\left(23^{\prime}\right)-\mathrm{H}(23 \mathrm{~F})$ & 109.5 \\
\hline$C\left(24^{\prime}\right)-C(22)-C\left(25^{\prime}\right)$ & $116.0(4)$ & $\mathrm{C}(22)-\mathrm{C}\left(24^{\prime}\right)-\mathrm{H}(24 \mathrm{D})$ & 109.5 \\
\hline $\mathrm{C}(24)-\mathrm{C}(22)-\mathrm{C}(25)$ & $112.8(3)$ & $\mathrm{C}(22)-\mathrm{C}\left(24^{\prime}\right)-\mathrm{H}(24 \mathrm{E})$ & 109.5 \\
\hline $\mathrm{C}\left(24^{\prime}\right)-\mathrm{C}(22)-\mathrm{C}(10)$ & $110.4(2)$ & $\mathrm{H}(24 \mathrm{D})-\mathrm{C}\left(24^{\prime}\right)-\mathrm{H}(24 \mathrm{E})$ & 109.5 \\
\hline$C\left(25^{\prime}\right)-C(22)-C(10)$ & $106.8(2)$ & $\mathrm{C}(22)-\mathrm{C}\left(24^{\prime}\right)-\mathrm{H}(24 \mathrm{~F})$ & 109.5 \\
\hline$C(24)-C(22)-C(10)$ & $111.2(2)$ & $\mathrm{H}(24 \mathrm{D})-\mathrm{C}\left(24^{\prime}\right)-\mathrm{H}(24 \mathrm{~F})$ & 109.5 \\
\hline$C(25)-C(22)-C(10)$ & $109.62(17)$ & $\mathrm{H}(24 \mathrm{E})-\mathrm{C}\left(24^{\prime}\right)-\mathrm{H}(24 \mathrm{~F})$ & 109.5 \\
\hline $\mathrm{C}(24)-\mathrm{C}(22)-\mathrm{C}(23)$ & $106.0(2)$ & $\mathrm{C}(22)-\mathrm{C}\left(25^{\prime}\right)-\mathrm{H}(25 \mathrm{D})$ & 109.5 \\
\hline$C(25)-C(22)-C(23)$ & $105.1(2)$ & $\mathrm{C}(22)-\mathrm{C}\left(25^{\prime}\right)-\mathrm{H}(25 \mathrm{E})$ & 109.5 \\
\hline$C(10)-C(22)-C(23)$ & $111.93(17)$ & $\mathrm{H}(25 \mathrm{D})-\mathrm{C}\left(25^{\prime}\right)-\mathrm{H}(25 \mathrm{E})$ & 109.5 \\
\hline $\mathrm{C}\left(24^{\prime}\right)-\mathrm{C}(22)-\mathrm{C}\left(23^{\prime}\right)$ & $108.0(3)$ & $\mathrm{C}(22)-\mathrm{C}\left(25^{\prime}\right)-\mathrm{H}(25 \mathrm{~F})$ & 109.5 \\
\hline $\mathrm{C}\left(25^{\prime}\right)-\mathrm{C}(22)-\mathrm{C}\left(23^{\prime}\right)$ & $105.2(3)$ & $\mathrm{H}(25 \mathrm{D})-\mathrm{C}\left(25^{\prime}\right)-\mathrm{H}(25 \mathrm{~F})$ & 109.5 \\
\hline $\mathrm{C}(10)-\mathrm{C}(22)-\mathrm{C}\left(23^{\prime}\right)$ & $110.3(2)$ & $\mathrm{H}(25 \mathrm{E})-\mathrm{C}\left(25^{\prime}\right)-\mathrm{H}(25 \mathrm{~F})$ & 109.5 \\
\hline $\mathrm{C}(22)-\mathrm{C}(23)-\mathrm{H}(23 \mathrm{~A})$ & 109.5 & $\mathrm{C}(29)-\mathrm{C}(26)-\mathrm{C}(12)$ & $112.43(14)$ \\
\hline $\mathrm{C}(22)-\mathrm{C}(23)-\mathrm{H}(23 \mathrm{~B})$ & 109.5 & $C(29)-C(26)-C(27)$ & $107.70(15)$ \\
\hline $\mathrm{H}(23 \mathrm{~A})-\mathrm{C}(23)-\mathrm{H}(23 \mathrm{~B})$ & 109.5 & $C(12)-C(26)-C(27)$ & $109.91(13)$ \\
\hline $\mathrm{C}(22)-\mathrm{C}(23)-\mathrm{H}(23 \mathrm{C})$ & 109.5 & $\mathrm{C}(29)-\mathrm{C}(26)-\mathrm{C}(28)$ & $108.60(14)$ \\
\hline $\mathrm{H}(23 \mathrm{~A})-\mathrm{C}(23)-\mathrm{H}(23 \mathrm{C})$ & 109.5 & $\mathrm{C}(12)-\mathrm{C}(26)-\mathrm{C}(28)$ & $108.49(14)$ \\
\hline $\mathrm{H}(23 \mathrm{~B})-\mathrm{C}(23)-\mathrm{H}(23 \mathrm{C})$ & 109.5 & $C(27)-C(26)-C(28)$ & $109.69(15)$ \\
\hline $\mathrm{C}(22)-\mathrm{C}(24)-\mathrm{H}(24 \mathrm{~A})$ & 109.5 & $\mathrm{C}(26)-\mathrm{C}(27)-\mathrm{H}(27 \mathrm{~A})$ & 109.5 \\
\hline $\mathrm{C}(22)-\mathrm{C}(24)-\mathrm{H}(24 \mathrm{~B})$ & 109.5 & $\mathrm{C}(26)-\mathrm{C}(27)-\mathrm{H}(27 \mathrm{~B})$ & 109.5 \\
\hline $\mathrm{H}(24 \mathrm{~A})-\mathrm{C}(24)-\mathrm{H}(24 \mathrm{~B})$ & 109.5 & $\mathrm{H}(27 \mathrm{~A})-\mathrm{C}(27)-\mathrm{H}(27 \mathrm{~B})$ & 109.5 \\
\hline $\mathrm{C}(22)-\mathrm{C}(24)-\mathrm{H}(24 \mathrm{C})$ & 109.5 & $\mathrm{C}(26)-\mathrm{C}(27)-\mathrm{H}(27 \mathrm{C})$ & 109.5 \\
\hline $\mathrm{H}(24 \mathrm{~A})-\mathrm{C}(24)-\mathrm{H}(24 \mathrm{C})$ & 109.5 & $\mathrm{H}(27 \mathrm{~A})-\mathrm{C}(27)-\mathrm{H}(27 \mathrm{C})$ & 109.5 \\
\hline $\mathrm{H}(24 \mathrm{~B})-\mathrm{C}(24)-\mathrm{H}(24 \mathrm{C})$ & 109.5 & $\mathrm{H}(27 \mathrm{~B})-\mathrm{C}(27)-\mathrm{H}(27 \mathrm{C})$ & 109.5 \\
\hline $\mathrm{C}(22)-\mathrm{C}(25)-\mathrm{H}(25 \mathrm{~A})$ & 109.5 & $\mathrm{C}(26)-\mathrm{C}(28)-\mathrm{H}(28 \mathrm{~A})$ & 109.5 \\
\hline $\mathrm{C}(22)-\mathrm{C}(25)-\mathrm{H}(25 \mathrm{~B})$ & 109.5 & $\mathrm{C}(26)-\mathrm{C}(28)-\mathrm{H}(28 \mathrm{~B})$ & 109.5 \\
\hline $\mathrm{H}(25 \mathrm{~A})-\mathrm{C}(25)-\mathrm{H}(25 \mathrm{~B})$ & 109.5 & $\mathrm{H}(28 \mathrm{~A})-\mathrm{C}(28)-\mathrm{H}(28 \mathrm{~B})$ & 109.5 \\
\hline $\mathrm{C}(22)-\mathrm{C}(25)-\mathrm{H}(25 \mathrm{C})$ & 109.5 & $\mathrm{C}(26)-\mathrm{C}(28)-\mathrm{H}(28 \mathrm{C})$ & 109.5 \\
\hline $\mathrm{H}(25 \mathrm{~A})-\mathrm{C}(25)-\mathrm{H}(25 \mathrm{C})$ & 109.5 & $\mathrm{H}(28 \mathrm{~A})-\mathrm{C}(28)-\mathrm{H}(28 \mathrm{C})$ & 109.5 \\
\hline $\mathrm{H}(25 \mathrm{~B})-\mathrm{C}(25)-\mathrm{H}(25 \mathrm{C})$ & 109.5 & $\mathrm{H}(28 \mathrm{~B})-\mathrm{C}(28)-\mathrm{H}(28 \mathrm{C})$ & 109.5 \\
\hline $\mathrm{C}(22)-\mathrm{C}\left(23^{\prime}\right)-\mathrm{H}(23 \mathrm{D})$ & 109.5 & $\mathrm{C}(26)-\mathrm{C}(29)-\mathrm{H}(29 \mathrm{~A})$ & 109.5 \\
\hline
\end{tabular}




$\begin{array}{llll}\mathrm{C}(26)-\mathrm{C}(29)-\mathrm{H}(29 \mathrm{~B}) & 109.5 & \mathrm{H}(29 \mathrm{~A})-\mathrm{C}(29)-\mathrm{H}(29 \mathrm{C}) & 109.5 \\ \mathrm{H}(29 \mathrm{~A})-\mathrm{C}(29)-\mathrm{H}(29 \mathrm{~B}) & 109.5 & \mathrm{H}(29 \mathrm{~B})-\mathrm{C}(29)-\mathrm{H}(29 \mathrm{C}) & 109.5 \\ \mathrm{C}(26)-\mathrm{C}(29)-\mathrm{H}(29 \mathrm{C}) & 109.5 & & \end{array}$

Symmetry transformations used to generate equivalent atoms:

$\# 1-\mathrm{x}+2,-\mathrm{y},-\mathrm{z}+1$ 
Table S4. Anisotropic displacement parameters $\left(\AA^{2} \times 10^{3}\right)$ for neijk01. The anisotropic displacement factor exponent takes the form: $-2 \pi^{2}\left[h^{2} a^{* 2} U_{11}+\ldots+2 h \mathrm{k} \mathrm{a}^{*} \mathrm{~b}^{*} \mathrm{U}_{12}\right]$

\begin{tabular}{|c|c|c|c|c|c|c|}
\hline & $\mathrm{U}_{11}$ & $\mathrm{U}_{22}$ & $\mathrm{U}_{33}$ & $\mathrm{U}_{23}$ & $\mathrm{U}_{13}$ & $\mathrm{U}_{12}$ \\
\hline P1 & $20(1)$ & $19(1)$ & $21(1)$ & $-2(1)$ & $5(1)$ & $-1(1)$ \\
\hline $\mathrm{C} 1$ & $18(1)$ & $17(1)$ & $23(1)$ & $-2(1)$ & $3(1)$ & $-1(1)$ \\
\hline $\mathrm{C} 2$ & $22(1)$ & 19(1) & $16(1)$ & $1(1)$ & $2(1)$ & $0(1)$ \\
\hline $\mathrm{C} 3$ & $21(1)$ & $22(1)$ & $17(1)$ & $0(1)$ & $4(1)$ & $-1(1)$ \\
\hline $\mathrm{C} 4$ & $26(1)$ & $20(1)$ & $15(1)$ & $2(1)$ & $2(1)$ & $-2(1)$ \\
\hline C5 & $30(1)$ & $17(1)$ & $19(1)$ & $2(1)$ & $4(1)$ & $3(1)$ \\
\hline C6 & $23(1)$ & $22(1)$ & $17(1)$ & $4(1)$ & $4(1)$ & $3(1)$ \\
\hline $\mathrm{C} 7$ & $21(1)$ & $20(1)$ & $19(1)$ & $2(1)$ & $4(1)$ & $-1(1)$ \\
\hline $\mathrm{C} 8$ & $22(1)$ & $19(1)$ & $20(1)$ & $-3(1)$ & $2(1)$ & $1(1)$ \\
\hline C9 & $32(1)$ & $20(1)$ & $22(1)$ & $0(1)$ & $1(1)$ & $3(1)$ \\
\hline $\mathrm{C} 10$ & $35(1)$ & $25(1)$ & $22(1)$ & $-3(1)$ & $-6(1)$ & $6(1)$ \\
\hline $\mathrm{C} 11$ & $25(1)$ & $28(1)$ & $29(1)$ & $-6(1)$ & $-8(1)$ & $0(1)$ \\
\hline $\mathrm{C} 12$ & 21(1) & $25(1)$ & $27(1)$ & $-4(1)$ & $0(1)$ & $-2(1)$ \\
\hline $\mathrm{C} 13$ & $21(1)$ & $23(1)$ & $20(1)$ & $-5(1)$ & $0(1)$ & $-1(1)$ \\
\hline C14 & $33(1)$ & $21(1)$ & $24(1)$ & $-2(1)$ & $6(1)$ & $-7(1)$ \\
\hline $\mathrm{C} 15$ & $58(2)$ & $39(2)$ & $31(2)$ & $-10(1)$ & $12(2)$ & $-22(2)$ \\
\hline C16 & $67(2)$ & $41(2)$ & $39(2)$ & $18(2)$ & $-7(2)$ & $-28(2)$ \\
\hline $\mathrm{C} 17$ & $39(2)$ & $33(2)$ & $93(3)$ & $-17(2)$ & $30(2)$ & $-18(1)$ \\
\hline $\mathrm{C} 15^{\prime}$ & $83(8)$ & $36(5)$ & $57(6)$ & $-21(4)$ & $37(5)$ & $-28(5)$ \\
\hline C16' & $57(5)$ & $31(4)$ & $28(3)$ & $6(3)$ & $5(3)$ & $-19(4)$ \\
\hline $\mathrm{C} 17^{\prime}$ & $38(4)$ & $38(4)$ & $88(7)$ & $23(5)$ & $-24(5)$ & $-20(3)$ \\
\hline $\mathrm{C} 18$ & $26(1)$ & $26(1)$ & $27(1)$ & $6(1)$ & $9(1)$ & $5(1)$ \\
\hline C19 & $23(1)$ & $43(1)$ & $35(1)$ & $6(1)$ & $5(1)$ & $7(1)$ \\
\hline $\mathrm{C} 20$ & $38(1)$ & $34(1)$ & $43(1)$ & $3(1)$ & $18(1)$ & $12(1)$ \\
\hline $\mathrm{C} 21$ & $33(1)$ & $40(1)$ & $29(1)$ & $9(1)$ & $13(1)$ & $7(1)$ \\
\hline $\mathrm{C} 22$ & $52(1)$ & $29(1)$ & $22(1)$ & 2(1) & $-12(1)$ & $-1(1)$ \\
\hline $\mathrm{C} 23$ & $78(3)$ & $47(2)$ & $17(1)$ & $8(1)$ & $-7(2)$ & $-10(2)$ \\
\hline $\mathrm{C} 24$ & $62(3)$ & $60(3)$ & $40(2)$ & $7(2)$ & $-24(2)$ & $11(2)$ \\
\hline $\mathrm{C} 25$ & $94(3)$ & $35(2)$ & $22(1)$ & $-3(1)$ & $-6(2)$ & $-12(2)$ \\
\hline C23' & $61(3)$ & $49(3)$ & $23(2)$ & $6(2)$ & $-17(2)$ & $-20(3)$ \\
\hline C24' & $60(3)$ & $80(5)$ & $20(2)$ & $-9(2)$ & $-3(2)$ & $10(3)$ \\
\hline
\end{tabular}




\begin{tabular}{lllllll}
$\mathrm{C} 25^{\prime}$ & $59(3)$ & $37(3)$ & $32(2)$ & $14(2)$ & $-14(2)$ & $-9(2)$ \\
$\mathrm{C} 26$ & $23(1)$ & $34(1)$ & $32(1)$ & $-4(1)$ & $1(1)$ & $-10(1)$ \\
$\mathrm{C} 27$ & $38(1)$ & $44(1)$ & $49(1)$ & $13(1)$ & $-1(1)$ & $-15(1)$ \\
$\mathrm{C} 28$ & $36(1)$ & $51(1)$ & $40(1)$ & $-13(1)$ & $14(1)$ & $-14(1)$ \\
$\mathrm{C} 29$ & $29(1)$ & $45(1)$ & $45(1)$ & $-11(1)$ & $3(1)$ & $-15(1)$ \\
\hline
\end{tabular}


Table S5. Hydrogen coordinates $\left(\times 10^{4}\right)$ and isotropic displacement parameters $\left(\AA^{2} \times 10^{3}\right)$ for neijk01.

\begin{tabular}{|c|c|c|c|c|}
\hline & $\mathrm{x}$ & $\mathrm{y}$ & $\mathrm{z}$ & $\mathrm{U}(\mathrm{eq})$ \\
\hline $\mathrm{H} 1 \mathrm{~A}$ & 9041 & 39 & 5181 & 23 \\
\hline H1B & 9504 & -644 & 4574 & 23 \\
\hline $\mathrm{H} 3$ & 10104 & 1669 & 3071 & 24 \\
\hline H5 & 8280 & 3004 & 4442 & 26 \\
\hline H7 & 7647 & 913 & 3878 & 24 \\
\hline H9 & 8346 & 120 & 387 & 30 \\
\hline H11 & 6150 & -1142 & 734 & 34 \\
\hline H13 & 7850 & -549 & 4010 & 26 \\
\hline $\mathrm{H} 15 \mathrm{~A}$ & 9431 & 3691 & 5056 & 63 \\
\hline H15B & 10433 & 3944 & 4772 & 63 \\
\hline $\mathrm{H} 15 \mathrm{C}$ & 10325 & 3240 & 5643 & 63 \\
\hline H16A & 8880 & 3696 & 2627 & 75 \\
\hline H16B & 9450 & 3270 & 1635 & 75 \\
\hline H16C & 9881 & 3973 & 2385 & 75 \\
\hline H17A & 11288 & 3289 & 3332 & 80 \\
\hline H17B & 10851 & 2621 & 2478 & 80 \\
\hline $\mathrm{H} 17 \mathrm{C}$ & 11183 & 2531 & 4037 & 80 \\
\hline H15D & 10153 & 4115 & 4378 & 84 \\
\hline $\mathrm{H} 15 \mathrm{E}$ & 9631 & 3615 & 5323 & 84 \\
\hline $\mathrm{H} 15 \mathrm{~F}$ & 9083 & 3929 & 3975 & 84 \\
\hline H16D & 10161 & 2813 & 1779 & 58 \\
\hline H16E & 10452 & 3619 & 2183 & 58 \\
\hline $\mathrm{H} 16 \mathrm{~F}$ & 9385 & 3415 & 1826 & 58 \\
\hline H17D & 11131 & 2422 & 3889 & 87 \\
\hline H17E & 10896 & 2743 & 5268 & 87 \\
\hline $\mathrm{H} 17 \mathrm{~F}$ & 11361 & 3243 & 4259 & 87 \\
\hline H19A & 6120 & 2066 & 2863 & 50 \\
\hline H19B & 5508 & 1883 & 4016 & 50 \\
\hline $\mathrm{H} 19 \mathrm{C}$ & 6195 & 1293 & 3555 & 50 \\
\hline $\mathrm{H} 20 \mathrm{~A}$ & 6682 & 3150 & 4203 & 56 \\
\hline
\end{tabular}




\begin{tabular}{|c|c|c|c|c|}
\hline H20B & 7160 & 3055 & 5706 & 56 \\
\hline $\mathrm{H} 20 \mathrm{C}$ & 6078 & 2910 & 5333 & 56 \\
\hline $\mathrm{H} 21 \mathrm{~A}$ & 6280 & 1704 & 6354 & 50 \\
\hline H21B & 7366 & 1838 & 6711 & 50 \\
\hline $\mathrm{H} 21 \mathrm{C}$ & 6998 & 1148 & 5864 & 50 \\
\hline $\mathrm{H} 23 \mathrm{~A}$ & 7683 & 444 & -1646 & 73 \\
\hline H23B & 7480 & -83 & -2897 & 73 \\
\hline $\mathrm{H} 23 \mathrm{C}$ & 8274 & -275 & -1706 & 73 \\
\hline $\mathrm{H} 24 \mathrm{~A}$ & 5543 & -354 & -1134 & 86 \\
\hline H24B & 5857 & -121 & -2525 & 86 \\
\hline $\mathrm{H} 24 \mathrm{C}$ & 6078 & 387 & -1250 & 86 \\
\hline $\mathrm{H} 25 \mathrm{~A}$ & 6466 & -1531 & -1351 & 77 \\
\hline H25B & 7538 & -1455 & -1503 & 77 \\
\hline $\mathrm{H} 25 \mathrm{C}$ & 6769 & -1230 & -2705 & 77 \\
\hline $\mathrm{H} 23 \mathrm{D}$ & 5833 & -951 & -2573 & 70 \\
\hline $\mathrm{H} 23 \mathrm{E}$ & 5500 & -786 & -1163 & 70 \\
\hline $\mathrm{H} 23 \mathrm{~F}$ & 6135 & -1470 & -1335 & 70 \\
\hline H24D & 7485 & -753 & -2826 & 81 \\
\hline $\mathrm{H} 24 \mathrm{E}$ & 7772 & -1281 & -1597 & 81 \\
\hline $\mathrm{H} 24 \mathrm{~F}$ & 8228 & -501 & -1612 & 81 \\
\hline $\mathrm{H} 25 \mathrm{D}$ & 6408 & 289 & -2503 & 66 \\
\hline $\mathrm{H} 25 \mathrm{E}$ & 7169 & 586 & -1351 & 66 \\
\hline $\mathrm{H} 25 \mathrm{~F}$ & 6148 & 412 & -1036 & 66 \\
\hline $\mathrm{H} 27 \mathrm{~A}$ & 6617 & -2090 & 4927 & 66 \\
\hline H27B & 7445 & -1532 & 4892 & 66 \\
\hline $\mathrm{H} 27 \mathrm{C}$ & 7293 & -2163 & 3816 & 66 \\
\hline $\mathrm{H} 28 \mathrm{~A}$ & 5522 & -1057 & 4860 & 62 \\
\hline H28B & 5445 & -497 & 3654 & 62 \\
\hline $\mathrm{H} 28 \mathrm{C}$ & 6335 & -493 & 4759 & 62 \\
\hline $\mathrm{H} 29 \mathrm{~A}$ & 5287 & -2085 & 3267 & 60 \\
\hline H29B & 5899 & -2140 & 2074 & 60 \\
\hline $\mathrm{H} 29 \mathrm{C}$ & 5161 & -1506 & 2090 & 60 \\
\hline
\end{tabular}


Table S6. Torsion angles $\left[{ }^{\circ}\right]$ for neijk01.

\begin{tabular}{|c|c|c|c|}
\hline C8-P1-C1-C1\#1 & $173.33(13)$ & C3-C4-C14-C16' & $68.6(5)$ \\
\hline C2-P1-C1-C1\#1 & $-81.81(14)$ & C5-C4-C14-C16' & $-108.5(5)$ \\
\hline C8-P1-C2-C7 & $31.62(13)$ & C3-C4-C14-C15 & $-134.5(2)$ \\
\hline C1-P1-C2-C7 & $-71.97(13)$ & C5-C4-C14-C15 & $48.4(3)$ \\
\hline C8-P1-C2-C3 & $-150.49(11)$ & C3-C4-C14-C17 & $-11.1(3)$ \\
\hline C1-P1-C2-C3 & 105.91(11) & C5-C4-C14-C17 & $171.7(3)$ \\
\hline $\mathrm{C} 7-\mathrm{C} 2-\mathrm{C} 3-\mathrm{C} 4$ & $0.6(2)$ & C3-C4-C14-C15' & $-166.7(6)$ \\
\hline P1-C2-C3-C4 & $-177.39(11)$ & C5-C4-C14-C15' & $16.2(6)$ \\
\hline $\mathrm{C} 2-\mathrm{C} 3-\mathrm{C} 4-\mathrm{C} 5$ & $-0.2(2)$ & C3-C4-C14-C16 & $107.7(3)$ \\
\hline C2-C3-C4-C14 & $-177.36(13)$ & C5-C4-C14-C16 & $-69.4(3)$ \\
\hline C3-C4-C5-C6 & $-0.1(2)$ & C3-C4-C14-C17' & $-50.7(6)$ \\
\hline C14-C4-C5-C6 & $177.09(13)$ & C5-C4-C14-C17' & $132.2(6)$ \\
\hline C4-C5-C6-C7 & $0.0(2)$ & C5-C6-C18-C20 & $6.1(2)$ \\
\hline C4-C5-C6-C18 & $178.56(13)$ & C7-C6-C18-C20 & $-175.37(14)$ \\
\hline $\mathrm{C} 3-\mathrm{C} 2-\mathrm{C} 7-\mathrm{C} 6$ & $-0.7(2)$ & C5-C6-C18-C19 & $126.32(15)$ \\
\hline P1-C2-C7-C6 & $177.12(11)$ & C7-C6-C18-C19 & $-55.13(18)$ \\
\hline C5-C6-C7-C2 & $0.4(2)$ & C5-C6-C18-C21 & $-114.30(16)$ \\
\hline C18-C6-C7-C2 & $-178.18(13)$ & C7-C6-C18-C21 & $64.25(18)$ \\
\hline C2-P1-C8-C13 & $-88.98(13)$ & C9-C10-C22-C24' & $59.3(3)$ \\
\hline C1-P1-C8-C13 & $13.12(14)$ & C11-C10-C22-C24' & $-119.9(3)$ \\
\hline C2-P1-C8-C9 & $95.61(12)$ & C9-C10-C22-C25' & $-67.6(3)$ \\
\hline C1-P1-C8-C9 & $-162.29(12)$ & $\mathrm{C} 11-\mathrm{C} 10-\mathrm{C} 22-\mathrm{C} 25^{\prime}$ & 113.2(3) \\
\hline C13-C8-C9-C10 & $0.3(2)$ & C9-C10-C22-C24 & $-114.9(2)$ \\
\hline P1-C8-C9-C10 & $175.92(12)$ & $\mathrm{C} 11-\mathrm{C} 10-\mathrm{C} 22-\mathrm{C} 24$ & $65.9(3)$ \\
\hline C8-C9-C10-C11 & $-0.8(2)$ & C9-C10-C22-C25 & $119.6(2)$ \\
\hline C8-C9-C10-C22 & $179.99(15)$ & $\mathrm{C} 11-\mathrm{C} 10-\mathrm{C} 22-\mathrm{C} 25$ & $-59.6(3)$ \\
\hline C9-C10-C11-C12 & $0.5(2)$ & C9-C10-C22-C23 & $3.4(3)$ \\
\hline $\mathrm{C} 22-\mathrm{C} 10-\mathrm{C} 11-\mathrm{C} 12$ & $179.77(15)$ & $\mathrm{C} 11-\mathrm{C} 10-\mathrm{C} 22-\mathrm{C} 23$ & $-175.8(2)$ \\
\hline C10-C11-C12-C13 & $0.2(2)$ & C9-C10-C22-C23' & $178.5(3)$ \\
\hline $\mathrm{C} 10-\mathrm{C} 11-\mathrm{C} 12-\mathrm{C} 26$ & $179.72(15)$ & C11-C10-C22-C23' & $-0.7(3)$ \\
\hline C9-C8-C13-C12 & $0.6(2)$ & C11-C12-C26-C29 & $9.2(2)$ \\
\hline P1-C8-C13-C12 & $-174.81(12)$ & C13-C12-C26-C29 & $-171.28(15)$ \\
\hline C11-C12-C13-C8 & $-0.8(2)$ & $\mathrm{C} 11-\mathrm{C} 12-\mathrm{C} 26-\mathrm{C} 27$ & $129.19(17)$ \\
\hline $\mathrm{C} 26-\mathrm{C} 12-\mathrm{C} 13-\mathrm{C} 8$ & $179.70(14)$ & C13-C12-C26-C27 & $-51.3(2)$ \\
\hline
\end{tabular}


Symmetry transformations used to generate equivalent atoms:

\#1 - $\mathrm{x}+2,-\mathrm{y},-\mathrm{z}+1$ 


\section{2 $\mathrm{FeCl}_{2}\left({ }^{\mathrm{tBu}} \mathrm{dppe}\right)$}

REFERENCE NUMBER: neijk04

CRYSTAL STRUCTURE REPORT

$\mathrm{C}_{66} \mathrm{H}_{104} \mathrm{Cl}_{2} \mathrm{Fe} \mathrm{O}_{4} \mathrm{P}_{2}$

or

$\mathrm{FeCl}_{2}(\mathrm{PP}) \cdot 2$ dioxane

Report prepared for:

J. Kneebone, Prof. M. Neidig

February 11, 2013

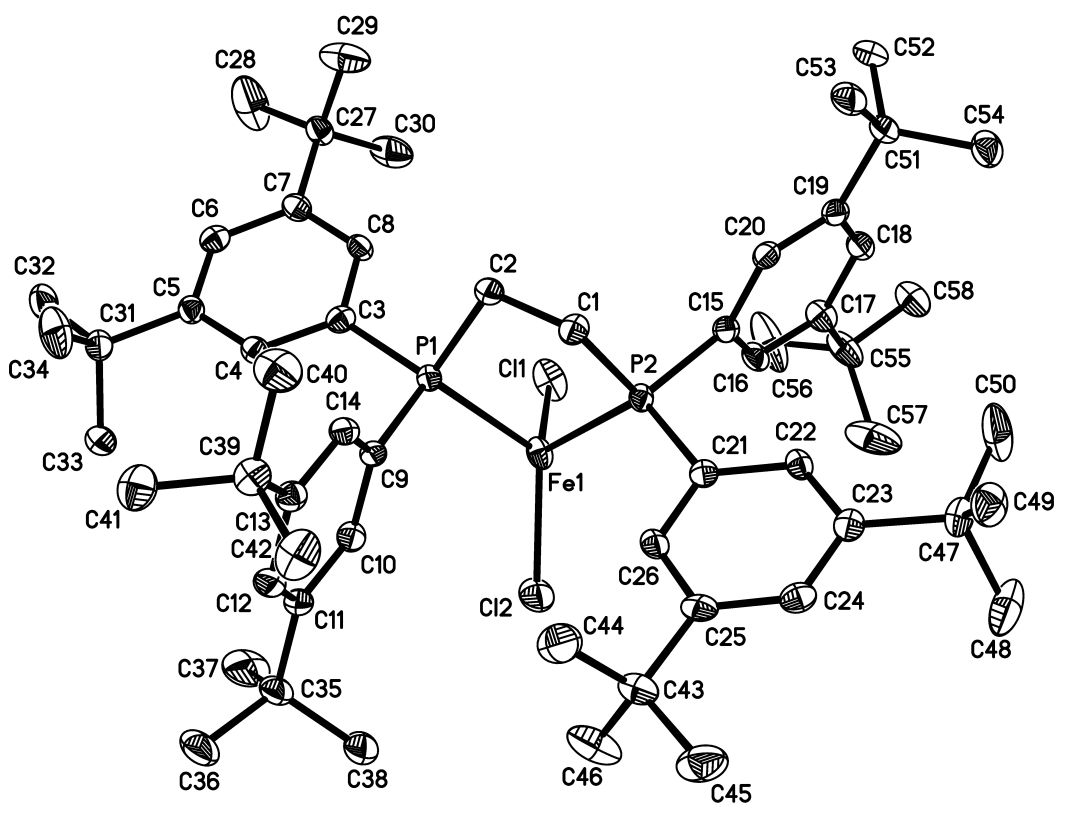

William W. Brennessel

X-ray Crystallographic Facility

Department of Chemistry, University of Rochester

120 Trustee Road

Rochester, NY 14627 


\section{Data collection}

A crystal $\left(0.48 \times 0.16 \times 0.12 \mathrm{~mm}^{3}\right)$ was placed onto the tip of a $0.1 \mathrm{~mm}$ diameter glass capillary tube or fiber and mounted on a Bruker SMART APEX II CCD Platform diffractometer for a data collection at 100.0(5) K. ${ }^{1}$ A preliminary set of cell constants and an orientation matrix were calculated from reflections harvested from three orthogonal wedges of reciprocal space. The full data collection was carried out using MoK $\alpha$ radiation (graphite monochromator) with a frame time of 90 seconds and a detector distance of $4.01 \mathrm{~cm}$. A randomly oriented region of reciprocal space was surveyed: three major sections of frames were collected with $0.50^{\circ}$ steps in $\omega$ at three different $\phi$ settings and a detector position of $-38^{\circ}$ in $2 \theta$. The intensity data were corrected for absorption. ${ }^{2}$ Final cell constants were calculated from the xyz centroids of 4038 strong reflections from the actual data collection after integration. ${ }^{3}$ See Table 1 for additional crystal and refinement information.

\section{Structure solution and refinement}

The structure was solved using SIR9 $7^{4}$ and refined using SHELXL-2012. ${ }^{5}$ The space group $P 2{ }_{1} / c$ was determined based on systematic absences. A direct-methods solution was calculated which provided most non-hydrogen atoms from the E-map. Full-matrix least squares / difference Fourier cycles were performed which located the remaining non-hydrogen atoms. All non-hydrogen atoms were refined with anisotropic displacement parameters. All hydrogen atoms were placed in ideal positions and refined as riding atoms with relative isotropic displacement parameters. The final full matrix least squares refinement converged to $R 1=0.0618\left(F^{2}, I>2 \sigma(I)\right)$ and $w R 2=$ $0.1649\left(F^{2}\right.$, all data).

\section{Structure description}

The structure is the one suggested, with all atoms in general positions. There are two cocrystallized dioxane solvent molecules per iron molecule. One tert-butyl group is modeled as disordered over two positions (89:11).

Unless noted otherwise all structural diagrams containing thermal displacement ellipsoids are drawn at the $50 \%$ probability level.

Data collection, structure solution, and structure refinement were conducted at the X-ray Crystallographic Facility, B51 Hutchison Hall, Department of Chemistry, University of Rochester. All publications arising from this report MUST either 1) include William W. Brennessel as a coauthor or 2) acknowledge William W. Brennessel and the Xray Crystallographic Facility of the Department of Chemistry at the University of Rochester. 
1 APEX2, version 2012.4-3; Bruker AXS: Madison, WI, 2012.

2 Sheldrick, G. M. SADABS, version 2008/1; University of Göttingen: Göttingen, Germany, 2008.

3 SAINT, version 7.68A; Bruker AXS: Madison, WI, 2009.

4 Altomare, A.; Burla, M. C.; Camalli, M.; Cascarano, G. L.; Giacovazzo, C.; Guagliardi, A.; Moliterni, A. G. G.;

Polidori, G.; Spagna, R. SIR97: A new program for solving and refining crystal structures; Istituto di Cristallografia, CNR: Bari, Italy, 1999.

5 Sheldrick, G. M. SHELXL-2012 University of Göttingen: Göttingen, Germany, 2012.

Some equations of interest:

$$
\begin{gathered}
R_{\mathrm{int}}=\Sigma\left|F_{\mathrm{o}}{ }^{2}-<F_{\mathrm{o}}{ }^{2}>\right| / \Sigma\left|F_{\mathrm{o}}{ }^{2}\right| \\
R 1=\Sigma|| F_{\mathrm{o}}|-| F_{\mathrm{c}} \| / \Sigma\left|F_{\mathrm{o}}\right| \\
w R 2=\left[\Sigma\left[w\left(F_{\mathrm{o}}{ }^{2}-F_{\mathrm{c}}{ }^{2}\right)^{2}\right] / \Sigma\left[w\left(F_{\mathrm{o}}{ }^{2}\right)^{2}\right]\right]^{1 / 2} \\
\text { where } w=1 /\left[\sigma^{2}\left(F_{\mathrm{o}}{ }^{2}\right)+(a P)^{2}+b P\right] \text { and } \\
P=1 / 3 \max \left(0, F_{\mathrm{o}}{ }^{2}\right)+2 / 3 F_{\mathrm{c}}{ }^{2} \\
\text { GOF }=S=\left[\Sigma\left[w\left(F_{\mathrm{o}}{ }^{2}-F_{\mathrm{c}}{ }^{2}\right)^{2}\right] /(m-n)\right]^{1 / 2}
\end{gathered}
$$

where $m=$ number of reflections and $n=$ number of parameters

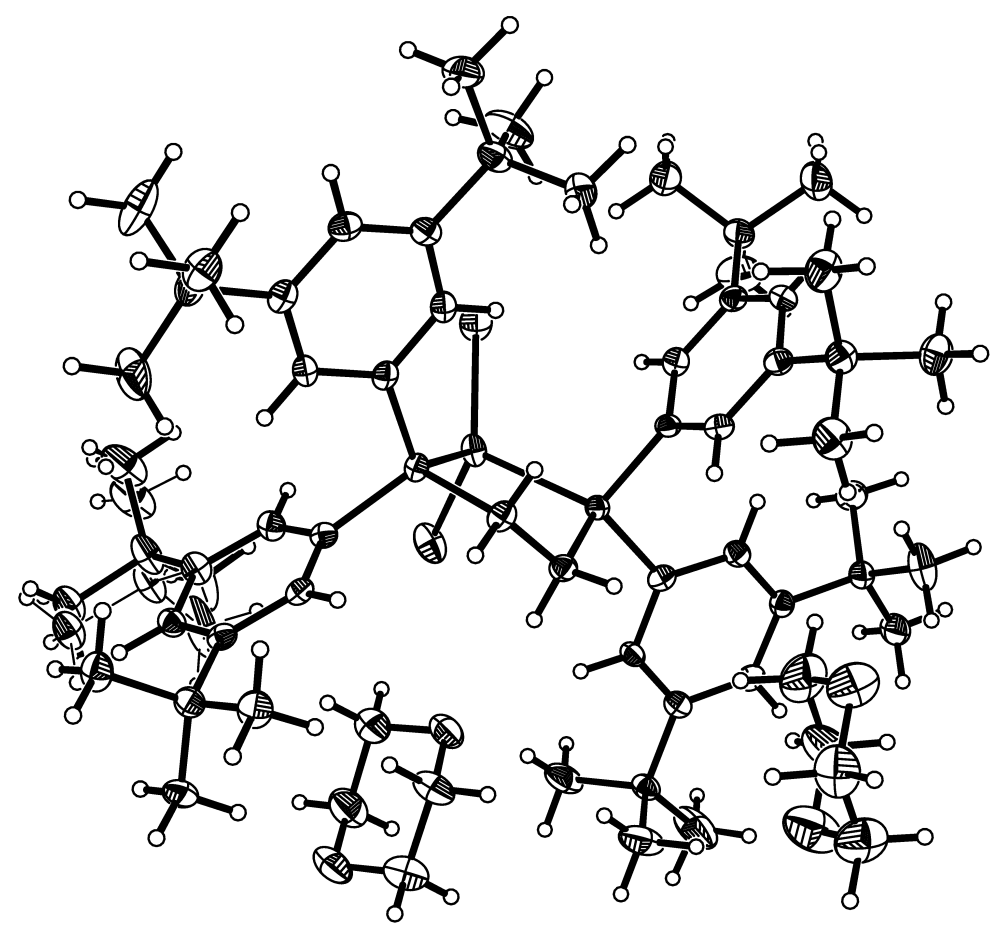


Table S7. Crystal data and structure refinement for neijk04.

\begin{tabular}{|c|c|c|}
\hline Identification code & \multicolumn{2}{|l|}{ neijk04 } \\
\hline Empirical formula & \multicolumn{2}{|c|}{$\mathrm{C} 66 \mathrm{H} 104 \mathrm{Cl} 2 \mathrm{Fe} \mathrm{O} 4 \mathrm{P} 2$} \\
\hline Formula weight & \multicolumn{2}{|l|}{1150.18} \\
\hline Temperature & \multicolumn{2}{|l|}{$100.0(5) \mathrm{K}$} \\
\hline Wavelength & \multicolumn{2}{|l|}{$0.71073 \AA$} \\
\hline Crystal system & \multicolumn{2}{|l|}{ monoclinic } \\
\hline Space group & \multicolumn{2}{|l|}{$P 2_{1} / c$} \\
\hline \multirow[t]{3}{*}{ Unit cell dimensions } & $a=14.135(4) \AA$ & $\alpha=90^{\circ}$ \\
\hline & $b=28.716(8) \AA$ & $\beta=102.479(6)^{\circ}$ \\
\hline & $c=16.870(5) \AA$ & $\gamma=90^{\circ}$ \\
\hline Volume & \multicolumn{2}{|l|}{$6686(3) \AA^{3}$} \\
\hline$Z$ & \multicolumn{2}{|l|}{4} \\
\hline Density (calculated) & \multicolumn{2}{|l|}{$1.143 \mathrm{Mg} / \mathrm{m}^{3}$} \\
\hline Absorption coefficient & \multicolumn{2}{|l|}{$0.396 \mathrm{~mm}^{-1}$} \\
\hline$F(000)$ & \multicolumn{2}{|l|}{2488} \\
\hline Crystal color, morphology & \multicolumn{2}{|l|}{ colorless, needle } \\
\hline Crystal size & \multicolumn{2}{|c|}{$0.48 \times 0.16 \times 0.12 \mathrm{~mm}^{3}$} \\
\hline Theta range for data collection & \multicolumn{2}{|l|}{1.425 to $29.574^{\circ}$} \\
\hline Index ranges & \multicolumn{2}{|c|}{$-19 \leq h \leq 19,-38 \leq k \leq 39,-23 \leq l \leq 23$} \\
\hline Reflections collected & \multicolumn{2}{|l|}{78114} \\
\hline Independent reflections & \multicolumn{2}{|c|}{$18751[R(\mathrm{int})=0.0998]$} \\
\hline Observed reflections & \multicolumn{2}{|l|}{10927} \\
\hline Completeness to theta $=29.575^{\circ}$ & \multicolumn{2}{|l|}{$99.9 \%$} \\
\hline Absorption correction & \multicolumn{2}{|l|}{ Multi-scan } \\
\hline Max. and min. transmission & \multicolumn{2}{|l|}{0.7461 and 0.6845} \\
\hline Refinement method & \multicolumn{2}{|c|}{ Full-matrix least-squares on $F^{2}$} \\
\hline Data / restraints / parameters & \multicolumn{2}{|l|}{$18751 / 7 / 713$} \\
\hline Goodness-of-fit on $F^{2}$ & \multicolumn{2}{|l|}{1.017} \\
\hline Final $R$ indices $[I>2 \operatorname{sigma}(I)]$ & \multicolumn{2}{|c|}{$R 1=0.0618, w R 2=0.1419$} \\
\hline$R$ indices (all data) & \multicolumn{2}{|c|}{$R 1=0.1193, w R 2=0.1649$} \\
\hline Largest diff. peak and hole & \multicolumn{2}{|c|}{1.687 and -0.786 e..$\AA^{-3}$} \\
\hline
\end{tabular}


Table S8. Atomic coordinates $\left(\mathrm{x} 10^{4}\right)$ and equivalent isotropic displacement parameters $\left(\AA^{2} \times 10^{3}\right)$ for neijk04. $U_{e q}$ is defined as one third of the trace of the orthogonalized $U_{i j}$ tensor.

\begin{tabular}{|c|c|c|c|c|}
\hline & $\mathrm{x}$ & $\mathrm{y}$ & $\mathrm{z}$ & $\mathrm{U}_{\mathrm{eq}}$ \\
\hline $\mathrm{Fe} 1$ & $7787(1)$ & $2417(1)$ & $5510(1)$ & $20(1)$ \\
\hline Cl1 & $9363(1)$ & $2543(1)$ & $5688(1)$ & $33(1)$ \\
\hline $\mathrm{Cl} 2$ & $6910(1)$ & 2231(1) & 4293(1) & $33(1)$ \\
\hline P1 & $7192(1)$ & $3029(1)$ & $6269(1)$ & $17(1)$ \\
\hline $\mathrm{P} 2$ & $7415(1)$ & 1921(1) & $6587(1)$ & $16(1)$ \\
\hline $\mathrm{C} 1$ & $6956(2)$ & $2306(1)$ & 7294(2) & $20(1)$ \\
\hline $\mathrm{C} 2$ & $7347(2)$ & 2801(1) & 7294(2) & 19(1) \\
\hline $\mathrm{C} 3$ & $7724(2)$ & $3607(1)$ & $6414(2)$ & $19(1)$ \\
\hline $\mathrm{C} 4$ & $7223(2)$ & 3993(1) & 6031(2) & 19(1) \\
\hline $\mathrm{C} 5$ & $7646(2)$ & $4433(1)$ & 6099(2) & $19(1)$ \\
\hline C6 & $8591(2)$ & $4477(1)$ & $6565(2)$ & 20(1) \\
\hline $\mathrm{C} 7$ & $9098(2)$ & 4098(1) & $6966(2)$ & $20(1)$ \\
\hline $\mathrm{C} 8$ & $8657(2)$ & $3660(1)$ & 6881(2) & $18(1)$ \\
\hline $\mathrm{C} 9$ & $5911(2)$ & $3136(1)$ & $5867(2)$ & $17(1)$ \\
\hline $\mathrm{C} 10$ & $5634(2)$ & $3160(1)$ & $5026(2)$ & $20(1)$ \\
\hline $\mathrm{C} 11$ & 4692(2) & $3282(1)$ & $4636(2)$ & $20(1)$ \\
\hline $\mathrm{C} 12$ & $4040(2)$ & $3362(1)$ & $5125(2)$ & $21(1)$ \\
\hline $\mathrm{C} 13$ & $4288(2)$ & $3336(1)$ & 5975(2) & 19(1) \\
\hline $\mathrm{C} 14$ & $5236(2)$ & $3225(1)$ & $6340(2)$ & $17(1)$ \\
\hline $\mathrm{C} 15$ & $8459(2)$ & $1605(1)$ & 7141(2) & $17(1)$ \\
\hline C16 & $9095(2)$ & $1436(1)$ & $6689(2)$ & $20(1)$ \\
\hline $\mathrm{C} 17$ & $9877(2)$ & $1155(1)$ & 7034(2) & $23(1)$ \\
\hline $\mathrm{C} 18$ & 9998(2) & $1048(1)$ & 7854(2) & $21(1)$ \\
\hline $\mathrm{C} 19$ & $9375(2)$ & $1214(1)$ & $8332(2)$ & $19(1)$ \\
\hline $\mathrm{C} 20$ & $8609(2)$ & 1493(1) & $7966(2)$ & $18(1)$ \\
\hline $\mathrm{C} 21$ & $6468(2)$ & $1484(1)$ & $6379(2)$ & $17(1)$ \\
\hline $\mathrm{C} 22$ & $6596(2)$ & 1033(1) & 6691(2) & $17(1)$ \\
\hline $\mathrm{C} 23$ & $5838(2)$ & $715(1)$ & $6546(2)$ & $19(1)$ \\
\hline $\mathrm{C} 24$ & $4942(2)$ & $860(1)$ & $6082(2)$ & $21(1)$ \\
\hline $\mathrm{C} 25$ & $4796(2)$ & $1304(1)$ & $5763(2)$ & $20(1)$ \\
\hline $\mathrm{C} 26$ & $5573(2)$ & $1615(1)$ & $5912(2)$ & 19(1) \\
\hline
\end{tabular}




\begin{tabular}{|c|c|c|c|c|}
\hline $\mathrm{C} 27$ & $10110(2)$ & $4146(1)$ & $7516(2)$ & $22(1)$ \\
\hline $\mathrm{C} 28$ & $10538(3)$ & $4627(1)$ & $7474(3)$ & $67(1)$ \\
\hline $\mathrm{C} 29$ & $10055(2)$ & 4044(1) & $8390(2)$ & $43(1)$ \\
\hline $\mathrm{C} 30$ & 10794(2) & $3795(1)$ & $7259(2)$ & $37(1)$ \\
\hline $\mathrm{C} 31$ & $7062(2)$ & $4846(1)$ & $5671(2)$ & $23(1)$ \\
\hline $\mathrm{C} 32$ & $7659(2)$ & $5286(1)$ & $5715(2)$ & $34(1)$ \\
\hline C33 & $6671(2)$ & $4735(1)$ & $4771(2)$ & $32(1)$ \\
\hline $\mathrm{C} 34$ & $6210(2)$ & $4938(1)$ & $6073(2)$ & $41(1)$ \\
\hline $\mathrm{C} 35$ & $4422(2)$ & $3310(1)$ & $3708(2)$ & $26(1)$ \\
\hline C36 & $3442(3)$ & $3550(1)$ & $3401(2)$ & $43(1)$ \\
\hline C37 & $5194(3)$ & $3584(1)$ & $3392(2)$ & $45(1)$ \\
\hline $\mathrm{C} 38$ & $4366(3)$ & $2818(1)$ & $3360(2)$ & $36(1)$ \\
\hline C39 & $3514(2)$ & $3437(1)$ & $6457(2)$ & $23(1)$ \\
\hline $\mathrm{C} 40$ & $3920(2)$ & $3423(1)$ & $7370(2)$ & $35(1)$ \\
\hline $\mathrm{C} 41$ & 3096(2) & $3924(1)$ & $6235(2)$ & $32(1)$ \\
\hline $\mathrm{C} 42$ & $2694(2)$ & $3075(1)$ & $6242(2)$ & $35(1)$ \\
\hline $\mathrm{C} 43$ & $3821(2)$ & $1472(1)$ & $5253(2)$ & $25(1)$ \\
\hline $\mathrm{C} 44$ & $3447(2)$ & $1865(1)$ & $5698(2)$ & $43(1)$ \\
\hline $\mathrm{C} 45$ & $3061(2)$ & $1080(1)$ & $5106(2)$ & $39(1)$ \\
\hline $\mathrm{C} 46$ & $3965(3)$ & $1625(1)$ & $4427(2)$ & $47(1)$ \\
\hline $\mathrm{C} 47$ & $5956(2)$ & $216(1)$ & $6881(2)$ & $24(1)$ \\
\hline $\mathrm{C} 48$ & $5793(3)$ & $-127(1)$ & $6171(2)$ & $45(1)$ \\
\hline C49 & $5213(2)$ & $122(1)$ & $7400(2)$ & $31(1)$ \\
\hline C50 & $6963(2)$ & $135(1)$ & $7414(2)$ & $46(1)$ \\
\hline C51 & $9554(2)$ & $1078(1)$ & $9232(2)$ & $23(1)$ \\
\hline $\mathrm{C} 52$ & $10550(2)$ & $1260(1)$ & $9670(2)$ & $31(1)$ \\
\hline $\mathrm{C} 53$ & $8787(2)$ & $1280(1)$ & $9646(2)$ & $29(1)$ \\
\hline $\mathrm{C} 54$ & $9526(2)$ & $545(1)$ & $9304(2)$ & $28(1)$ \\
\hline $\mathrm{C} 55$ & $10550(4)$ & $989(2)$ & $6490(3)$ & $31(1)$ \\
\hline C56 & $10989(5)$ & $1426(2)$ & $6172(5)$ & $55(2)$ \\
\hline C57 & $9988(6)$ & $700(3)$ & $5784(3)$ & $56(2)$ \\
\hline C58 & 11385(4) & $693(2)$ & $6958(4)$ & $32(1)$ \\
\hline C55' & $10785(19)$ & $1062(13)$ & $6700(20)$ & $31(1)$ \\
\hline C56' & $11340(40)$ & $1515(13)$ & $6570(40)$ & $55(2)$ \\
\hline C57' & $10370(40)$ & $841(19)$ & $5880(20)$ & $56(2)$ \\
\hline C58' & $11540(30)$ & $735(15)$ & $7190(30)$ & $32(1)$ \\
\hline
\end{tabular}




\begin{tabular}{lllll} 
O1 & $6022(2)$ & $4123(1)$ & $8666(2)$ & $51(1)$ \\
C59 & $6532(3)$ & $4111(1)$ & $9495(2)$ & $49(1)$ \\
C60 & $7258(3)$ & $4488(2)$ & $9657(2)$ & $54(1)$ \\
O2 & $7931(2)$ & $4449(1)$ & $9153(2)$ & $67(1)$ \\
C61 & $7428(3)$ & $4455(2)$ & $8337(2)$ & $52(1)$ \\
C62 & $6698(3)$ & $4079(2)$ & $8158(2)$ & $56(1)$ \\
O3 & $9884(2)$ & $2731(1)$ & $8017(1)$ & $34(1)$ \\
C63 & $9928(2)$ & $2641(1)$ & $8855(2)$ & $34(1)$ \\
C64 & $10938(2)$ & $2722(1)$ & $9351(2)$ & $40(1)$ \\
O4 & $11630(2)$ & $2441(1)$ & $9066(2)$ & $42(1)$ \\
C65 & $11573(2)$ & $2533(1)$ & $8236(2)$ & $45(1)$ \\
C66 & $10573(2)$ & $2441(1)$ & $7748(2)$ & $42(1)$ \\
& & & & \\
\hline
\end{tabular}


Table S9. Bond lengths $[\AA]$ and angles $\left[{ }^{\circ}\right]$ for neijk04.

\begin{tabular}{|c|c|c|c|}
\hline $\mathrm{Fe}(1)-\mathrm{Cl}(1)$ & $2.2120(10)$ & $C(13)-C(14)$ & $1.386(4)$ \\
\hline $\mathrm{Fe}(1)-\mathrm{Cl}(2)$ & $2.2225(10)$ & $\mathrm{C}(13)-\mathrm{C}(39)$ & $1.526(4)$ \\
\hline $\mathrm{Fe}(1)-\mathrm{P}(1)$ & $2.4291(9)$ & $\mathrm{C}(14)-\mathrm{H}(14)$ & 0.9500 \\
\hline $\mathrm{Fe}(1)-\mathrm{P}(2)$ & $2.4549(9)$ & $C(15)-C(16)$ & $1.386(4)$ \\
\hline $\mathrm{P}(1)-\mathrm{C}(9)$ & $1.816(3)$ & $C(15)-C(20)$ & $1.400(4)$ \\
\hline $\mathrm{P}(1)-\mathrm{C}(3)$ & $1.818(3)$ & $C(16)-C(17)$ & $1.390(4)$ \\
\hline $\mathrm{P}(1)-\mathrm{C}(2)$ & $1.818(3)$ & $\mathrm{C}(16)-\mathrm{H}(16)$ & 0.9500 \\
\hline $\mathrm{P}(2)-\mathrm{C}(15)$ & $1.810(3)$ & $\mathrm{C}(17)-\mathrm{C}(18)$ & $1.390(4)$ \\
\hline $\mathrm{P}(2)-\mathrm{C}(21)$ & $1.811(3)$ & $C(17)-C(55)$ & $1.533(4)$ \\
\hline $\mathrm{P}(2)-\mathrm{C}(1)$ & $1.845(3)$ & $\mathrm{C}(17)-\mathrm{C}\left(55^{\prime}\right)$ & $1.537(10)$ \\
\hline$C(1)-C(2)$ & $1.526(4)$ & $\mathrm{C}(18)-\mathrm{C}(19)$ & $1.399(4)$ \\
\hline $\mathrm{C}(1)-\mathrm{H}(1 \mathrm{~A})$ & 0.9900 & $\mathrm{C}(18)-\mathrm{H}(18)$ & 0.9500 \\
\hline C(1)-H(1B) & 0.9900 & $C(19)-C(20)$ & $1.382(4)$ \\
\hline $\mathrm{C}(2)-\mathrm{H}(2 \mathrm{~A})$ & 0.9900 & $C(19)-C(51)$ & $1.534(4)$ \\
\hline $\mathrm{C}(2)-\mathrm{H}(2 \mathrm{~B})$ & 0.9900 & $\mathrm{C}(20)-\mathrm{H}(20)$ & 0.9500 \\
\hline $\mathrm{C}(3)-\mathrm{C}(8)$ & $1.390(3)$ & $\mathrm{C}(21)-\mathrm{C}(26)$ & $1.390(4)$ \\
\hline$C(3)-C(4)$ & $1.396(4)$ & $C(21)-C(22)$ & $1.396(4)$ \\
\hline$C(4)-C(5)$ & $1.392(4)$ & $\mathrm{C}(22)-\mathrm{C}(23)$ & $1.388(4)$ \\
\hline $\mathrm{C}(4)-\mathrm{H}(4)$ & 0.9500 & $\mathrm{C}(22)-\mathrm{H}(22)$ & 0.9500 \\
\hline$C(5)-C(6)$ & $1.402(4)$ & $C(23)-C(24)$ & $1.400(4)$ \\
\hline$C(5)-C(31)$ & $1.533(4)$ & $\mathrm{C}(23)-\mathrm{C}(47)$ & $1.537(4)$ \\
\hline$C(6)-C(7)$ & $1.395(4)$ & $C(24)-C(25)$ & $1.383(4)$ \\
\hline $\mathrm{C}(6)-\mathrm{H}(6)$ & 0.9500 & $\mathrm{C}(24)-\mathrm{H}(24)$ & 0.9500 \\
\hline$C(7)-C(8)$ & $1.396(4)$ & $C(25)-C(26)$ & $1.395(4)$ \\
\hline$C(7)-C(27)$ & $1.534(4)$ & $C(25)-C(43)$ & $1.536(4)$ \\
\hline $\mathrm{C}(8)-\mathrm{H}(8)$ & 0.9500 & $\mathrm{C}(26)-\mathrm{H}(26)$ & 0.9500 \\
\hline$C(9)-C(10)$ & $1.390(4)$ & $\mathrm{C}(27)-\mathrm{C}(28)$ & $1.515(4)$ \\
\hline $\mathrm{C}(9)-\mathrm{C}(14)$ & $1.394(4)$ & $C(27)-C(29)$ & $1.521(4)$ \\
\hline$C(10)-C(11)$ & $1.397(4)$ & $\mathrm{C}(27)-\mathrm{C}(30)$ & $1.525(4)$ \\
\hline $\mathrm{C}(10)-\mathrm{H}(10)$ & 0.9500 & $\mathrm{C}(28)-\mathrm{H}(28 \mathrm{~A})$ & 0.9800 \\
\hline $\mathrm{C}(11)-\mathrm{C}(12)$ & $1.382(4)$ & $\mathrm{C}(28)-\mathrm{H}(28 \mathrm{~B})$ & 0.9800 \\
\hline C(11)-C(35) & $1.531(4)$ & $\mathrm{C}(28)-\mathrm{H}(28 \mathrm{C})$ & 0.9800 \\
\hline$C(12)-C(13)$ & $1.402(4)$ & C(29)-H(29A) & 0.9800 \\
\hline $\mathrm{C}(12)-\mathrm{H}(12)$ & 0.9500 & C(29)-H(29B) & 0.9800 \\
\hline
\end{tabular}




\begin{tabular}{|c|c|c|c|}
\hline $\mathrm{C}(29)-\mathrm{H}(29 \mathrm{C})$ & 0.9800 & $\mathrm{C}(41)-\mathrm{H}(41 \mathrm{C})$ & 0.9800 \\
\hline $\mathrm{C}(30)-\mathrm{H}(30 \mathrm{~A})$ & 0.9800 & $\mathrm{C}(42)-\mathrm{H}(42 \mathrm{~A})$ & 0.9800 \\
\hline $\mathrm{C}(30)-\mathrm{H}(30 \mathrm{~B})$ & 0.9800 & $\mathrm{C}(42)-\mathrm{H}(42 \mathrm{~B})$ & 0.9800 \\
\hline $\mathrm{C}(30)-\mathrm{H}(30 \mathrm{C})$ & 0.9800 & $\mathrm{C}(42)-\mathrm{H}(42 \mathrm{C})$ & 0.9800 \\
\hline$C(31)-C(32)$ & $1.513(4)$ & $C(43)-C(44)$ & $1.513(4)$ \\
\hline$C(31)-C(34)$ & $1.527(4)$ & $C(43)-C(46)$ & $1.517(4)$ \\
\hline $\mathrm{C}(31)-\mathrm{C}(33)$ & $1.534(4)$ & $C(43)-C(45)$ & $1.540(4)$ \\
\hline $\mathrm{C}(32)-\mathrm{H}(32 \mathrm{~A})$ & 0.9800 & $\mathrm{C}(44)-\mathrm{H}(44 \mathrm{~A})$ & 0.9800 \\
\hline $\mathrm{C}(32)-\mathrm{H}(32 \mathrm{~B})$ & 0.9800 & $\mathrm{C}(44)-\mathrm{H}(44 \mathrm{~B})$ & 0.9800 \\
\hline $\mathrm{C}(32)-\mathrm{H}(32 \mathrm{C})$ & 0.9800 & $\mathrm{C}(44)-\mathrm{H}(44 \mathrm{C})$ & 0.9800 \\
\hline $\mathrm{C}(33)-\mathrm{H}(33 \mathrm{~A})$ & 0.9800 & $\mathrm{C}(45)-\mathrm{H}(45 \mathrm{~A})$ & 0.9800 \\
\hline $\mathrm{C}(33)-\mathrm{H}(33 \mathrm{~B})$ & 0.9800 & $\mathrm{C}(45)-\mathrm{H}(45 \mathrm{~B})$ & 0.9800 \\
\hline $\mathrm{C}(33)-\mathrm{H}(33 \mathrm{C})$ & 0.9800 & $\mathrm{C}(45)-\mathrm{H}(45 \mathrm{C})$ & 0.9800 \\
\hline $\mathrm{C}(34)-\mathrm{H}(34 \mathrm{~A})$ & 0.9800 & $\mathrm{C}(46)-\mathrm{H}(46 \mathrm{~A})$ & 0.9800 \\
\hline $\mathrm{C}(34)-\mathrm{H}(34 \mathrm{~B})$ & 0.9800 & $\mathrm{C}(46)-\mathrm{H}(46 \mathrm{~B})$ & 0.9800 \\
\hline $\mathrm{C}(34)-\mathrm{H}(34 \mathrm{C})$ & 0.9800 & $\mathrm{C}(46)-\mathrm{H}(46 \mathrm{C})$ & 0.9800 \\
\hline $\mathrm{C}(35)-\mathrm{C}(38)$ & $1.525(4)$ & $C(47)-C(50)$ & $1.528(4)$ \\
\hline$C(35)-C(37)$ & $1.533(4)$ & $C(47)-C(48)$ & $1.530(4)$ \\
\hline$C(35)-C(36)$ & $1.534(4)$ & $C(47)-C(49)$ & $1.531(4)$ \\
\hline $\mathrm{C}(36)-\mathrm{H}(36 \mathrm{~A})$ & 0.9800 & $\mathrm{C}(48)-\mathrm{H}(48 \mathrm{~A})$ & 0.9800 \\
\hline $\mathrm{C}(36)-\mathrm{H}(36 \mathrm{~B})$ & 0.9800 & $\mathrm{C}(48)-\mathrm{H}(48 \mathrm{~B})$ & 0.9800 \\
\hline $\mathrm{C}(36)-\mathrm{H}(36 \mathrm{C})$ & 0.9800 & $\mathrm{C}(48)-\mathrm{H}(48 \mathrm{C})$ & 0.9800 \\
\hline $\mathrm{C}(37)-\mathrm{H}(37 \mathrm{~A})$ & 0.9800 & $\mathrm{C}(49)-\mathrm{H}(49 \mathrm{~A})$ & 0.9800 \\
\hline $\mathrm{C}(37)-\mathrm{H}(37 \mathrm{~B})$ & 0.9800 & $\mathrm{C}(49)-\mathrm{H}(49 \mathrm{~B})$ & 0.9800 \\
\hline $\mathrm{C}(37)-\mathrm{H}(37 \mathrm{C})$ & 0.9800 & $\mathrm{C}(49)-\mathrm{H}(49 \mathrm{C})$ & 0.9800 \\
\hline $\mathrm{C}(38)-\mathrm{H}(38 \mathrm{~A})$ & 0.9800 & $\mathrm{C}(50)-\mathrm{H}(50 \mathrm{~A})$ & 0.9800 \\
\hline $\mathrm{C}(38)-\mathrm{H}(38 \mathrm{~B})$ & 0.9800 & $\mathrm{C}(50)-\mathrm{H}(50 \mathrm{~B})$ & 0.9800 \\
\hline $\mathrm{C}(38)-\mathrm{H}(38 \mathrm{C})$ & 0.9800 & $\mathrm{C}(50)-\mathrm{H}(50 \mathrm{C})$ & 0.9800 \\
\hline$C(39)-C(40)$ & $1.523(4)$ & $C(51)-C(53)$ & $1.525(4)$ \\
\hline$C(39)-C(41)$ & $1.534(4)$ & $\mathrm{C}(51)-\mathrm{C}(52)$ & $1.533(4)$ \\
\hline$C(39)-C(42)$ & $1.541(4)$ & $\mathrm{C}(51)-\mathrm{C}(54)$ & $1.537(4)$ \\
\hline $\mathrm{C}(40)-\mathrm{H}(40 \mathrm{~A})$ & 0.9800 & $\mathrm{C}(52)-\mathrm{H}(52 \mathrm{~A})$ & 0.9800 \\
\hline $\mathrm{C}(40)-\mathrm{H}(40 \mathrm{~B})$ & 0.9800 & $\mathrm{C}(52)-\mathrm{H}(52 \mathrm{~B})$ & 0.9800 \\
\hline $\mathrm{C}(40)-\mathrm{H}(40 \mathrm{C})$ & 0.9800 & $\mathrm{C}(52)-\mathrm{H}(52 \mathrm{C})$ & 0.9800 \\
\hline $\mathrm{C}(41)-\mathrm{H}(41 \mathrm{~A})$ & 0.9800 & $\mathrm{C}(53)-\mathrm{H}(53 \mathrm{~A})$ & 0.9800 \\
\hline $\mathrm{C}(41)-\mathrm{H}(41 \mathrm{~B})$ & 0.9800 & $\mathrm{C}(53)-\mathrm{H}(53 \mathrm{~B})$ & 0.9800 \\
\hline
\end{tabular}




\begin{tabular}{|c|c|c|c|}
\hline $\mathrm{C}(53)-\mathrm{H}(53 \mathrm{C})$ & 0.9800 & $\mathrm{O}(2)-\mathrm{C}(61)$ & $1.407(4)$ \\
\hline $\mathrm{C}(54)-\mathrm{H}(54 \mathrm{~A})$ & 0.9800 & $\mathrm{C}(61)-\mathrm{C}(62)$ & $1.479(6)$ \\
\hline $\mathrm{C}(54)-\mathrm{H}(54 \mathrm{~B})$ & 0.9800 & $\mathrm{C}(61)-\mathrm{H}(61 \mathrm{~A})$ & 0.9900 \\
\hline $\mathrm{C}(54)-\mathrm{H}(54 \mathrm{C})$ & 0.9800 & $\mathrm{C}(61)-\mathrm{H}(61 \mathrm{~B})$ & 0.9900 \\
\hline$C(55)-C(57)$ & $1.527(6)$ & $\mathrm{C}(62)-\mathrm{H}(62 \mathrm{~A})$ & 0.9900 \\
\hline $\mathrm{C}(55)-\mathrm{C}(58)$ & $1.529(5)$ & $\mathrm{C}(62)-\mathrm{H}(62 \mathrm{~B})$ & 0.9900 \\
\hline$C(55)-C(56)$ & $1.546(5)$ & $\mathrm{O}(3)-\mathrm{C}(63)$ & $1.425(4)$ \\
\hline $\mathrm{C}(56)-\mathrm{H}(56 \mathrm{~A})$ & 0.9800 & $\mathrm{O}(3)-\mathrm{C}(66)$ & $1.428(4)$ \\
\hline $\mathrm{C}(56)-\mathrm{H}(56 \mathrm{~B})$ & 0.9800 & $C(63)-C(64)$ & $1.508(4)$ \\
\hline $\mathrm{C}(56)-\mathrm{H}(56 \mathrm{C})$ & 0.9800 & $\mathrm{C}(63)-\mathrm{H}(63 \mathrm{~A})$ & 0.9900 \\
\hline $\mathrm{C}(57)-\mathrm{H}(57 \mathrm{~A})$ & 0.9800 & $\mathrm{C}(63)-\mathrm{H}(63 \mathrm{~B})$ & 0.9900 \\
\hline $\mathrm{C}(57)-\mathrm{H}(57 \mathrm{~B})$ & 0.9800 & $\mathrm{C}(64)-\mathrm{O}(4)$ & $1.430(4)$ \\
\hline $\mathrm{C}(57)-\mathrm{H}(57 \mathrm{C})$ & 0.9800 & $\mathrm{C}(64)-\mathrm{H}(64 \mathrm{~A})$ & 0.9900 \\
\hline $\mathrm{C}(58)-\mathrm{H}(58 \mathrm{~A})$ & 0.9800 & $\mathrm{C}(64)-\mathrm{H}(64 \mathrm{~B})$ & 0.9900 \\
\hline $\mathrm{C}(58)-\mathrm{H}(58 \mathrm{~B})$ & 0.9800 & $\mathrm{O}(4)-\mathrm{C}(65)$ & $1.410(4)$ \\
\hline $\mathrm{C}(58)-\mathrm{H}(58 \mathrm{C})$ & 0.9800 & $C(65)-C(66)$ & $1.498(5)$ \\
\hline$C\left(55^{\prime}\right)-C\left(57^{\prime}\right)$ & $1.509(19)$ & $\mathrm{C}(65)-\mathrm{H}(65 \mathrm{~A})$ & 0.9900 \\
\hline $\mathrm{C}\left(55^{\prime}\right)-\mathrm{C}\left(58^{\prime}\right)$ & $1.532(18)$ & $\mathrm{C}(65)-\mathrm{H}(65 \mathrm{~B})$ & 0.9900 \\
\hline $\mathrm{C}\left(55^{\prime}\right)-\mathrm{C}\left(56^{\prime}\right)$ & $1.555(19)$ & $\mathrm{C}(66)-\mathrm{H}(66 \mathrm{~A})$ & 0.9900 \\
\hline $\mathrm{C}\left(56^{\prime}\right)-\mathrm{H}(56 \mathrm{D})$ & 0.9800 & $\mathrm{C}(66)-\mathrm{H}(66 \mathrm{~B})$ & 0.9900 \\
\hline $\mathrm{C}\left(56^{\prime}\right)-\mathrm{H}(56 \mathrm{E})$ & 0.9800 & $\mathrm{Cl}(1)-\mathrm{Fe}(1)-\mathrm{Cl}(2)$ & $120.65(4)$ \\
\hline $\mathrm{C}\left(56^{\prime}\right)-\mathrm{H}(56 \mathrm{~F})$ & 0.9800 & $\mathrm{Cl}(1)-\mathrm{Fe}(1)-\mathrm{P}(1)$ & $105.28(3)$ \\
\hline $\mathrm{C}\left(57^{\prime}\right)-\mathrm{H}(57 \mathrm{D})$ & 0.9800 & $\mathrm{Cl}(2)-\mathrm{Fe}(1)-\mathrm{P}(1)$ & $118.25(3)$ \\
\hline $\mathrm{C}\left(57^{\prime}\right)-\mathrm{H}(57 \mathrm{E})$ & 0.9800 & $\mathrm{Cl}(1)-\mathrm{Fe}(1)-\mathrm{P}(2)$ & $111.39(3)$ \\
\hline $\mathrm{C}\left(57^{\prime}\right)-\mathrm{H}(57 \mathrm{~F})$ & 0.9800 & $\mathrm{Cl}(2)-\mathrm{Fe}(1)-\mathrm{P}(2)$ & $112.20(4)$ \\
\hline $\mathrm{C}\left(58^{\prime}\right)-\mathrm{H}(58 \mathrm{D})$ & 0.9800 & $\mathrm{P}(1)-\mathrm{Fe}(1)-\mathrm{P}(2)$ & $82.85(3)$ \\
\hline $\mathrm{C}\left(58^{\prime}\right)-\mathrm{H}(58 \mathrm{E})$ & 0.9800 & $\mathrm{C}(9)-\mathrm{P}(1)-\mathrm{C}(3)$ & $104.28(12)$ \\
\hline $\mathrm{C}\left(58^{\prime}\right)-\mathrm{H}(58 \mathrm{~F})$ & 0.9800 & $\mathrm{C}(9)-\mathrm{P}(1)-\mathrm{C}(2)$ & $108.75(12)$ \\
\hline $\mathrm{O}(1)-\mathrm{C}(62)$ & $1.419(4)$ & $\mathrm{C}(3)-\mathrm{P}(1)-\mathrm{C}(2)$ & $103.83(12)$ \\
\hline $\mathrm{O}(1)-\mathrm{C}(59)$ & $1.429(4)$ & $\mathrm{C}(9)-\mathrm{P}(1)-\mathrm{Fe}(1)$ & $110.86(9)$ \\
\hline$C(59)-C(60)$ & $1.475(5)$ & $\mathrm{C}(3)-\mathrm{P}(1)-\mathrm{Fe}(1)$ & $123.44(9)$ \\
\hline $\mathrm{C}(59)-\mathrm{H}(59 \mathrm{~A})$ & 0.9900 & $\mathrm{C}(2)-\mathrm{P}(1)-\mathrm{Fe}(1)$ & $104.90(9)$ \\
\hline $\mathrm{C}(59)-\mathrm{H}(59 \mathrm{~B})$ & 0.9900 & $\mathrm{C}(15)-\mathrm{P}(2)-\mathrm{C}(21)$ & $103.85(12)$ \\
\hline $\mathrm{C}(60)-\mathrm{O}(2)$ & $1.410(4)$ & $\mathrm{C}(15)-\mathrm{P}(2)-\mathrm{C}(1)$ & $109.18(13)$ \\
\hline $\mathrm{C}(60)-\mathrm{H}(60 \mathrm{~A})$ & 0.9900 & $\mathrm{C}(21)-\mathrm{P}(2)-\mathrm{C}(1)$ & $101.05(12)$ \\
\hline $\mathrm{C}(60)-\mathrm{H}(60 \mathrm{~B})$ & 0.9900 & $\mathrm{C}(15)-\mathrm{P}(2)-\mathrm{Fe}(1)$ & $113.05(9)$ \\
\hline
\end{tabular}




\begin{tabular}{|c|c|c|c|}
\hline $\mathrm{C}(21)-\mathrm{P}(2)-\mathrm{Fe}(1)$ & $121.71(9)$ & $\mathrm{C}(9)-\mathrm{C}(10)-\mathrm{H}(10)$ & 119.3 \\
\hline $\mathrm{C}(1)-\mathrm{P}(2)-\mathrm{Fe}(1)$ & $107.00(9)$ & $\mathrm{C}(11)-\mathrm{C}(10)-\mathrm{H}(10)$ & 119.3 \\
\hline $\mathrm{C}(2)-\mathrm{C}(1)-\mathrm{P}(2)$ & $112.36(18)$ & $\mathrm{C}(12)-\mathrm{C}(11)-\mathrm{C}(10)$ & $116.9(2)$ \\
\hline $\mathrm{C}(2)-\mathrm{C}(1)-\mathrm{H}(1 \mathrm{~A})$ & 109.1 & $\mathrm{C}(12)-\mathrm{C}(11)-\mathrm{C}(35)$ & $123.4(2)$ \\
\hline $\mathrm{P}(2)-\mathrm{C}(1)-\mathrm{H}(1 \mathrm{~A})$ & 109.1 & $\mathrm{C}(10)-\mathrm{C}(11)-\mathrm{C}(35)$ & $119.7(2)$ \\
\hline $\mathrm{C}(2)-\mathrm{C}(1)-\mathrm{H}(1 \mathrm{~B})$ & 109.1 & $\mathrm{C}(11)-\mathrm{C}(12)-\mathrm{C}(13)$ & $123.3(2)$ \\
\hline $\mathrm{P}(2)-\mathrm{C}(1)-\mathrm{H}(1 \mathrm{~B})$ & 109.1 & $\mathrm{C}(11)-\mathrm{C}(12)-\mathrm{H}(12)$ & 118.4 \\
\hline $\mathrm{H}(1 \mathrm{~A})-\mathrm{C}(1)-\mathrm{H}(1 \mathrm{~B})$ & 107.9 & $\mathrm{C}(13)-\mathrm{C}(12)-\mathrm{H}(12)$ & 118.4 \\
\hline $\mathrm{C}(1)-\mathrm{C}(2)-\mathrm{P}(1)$ & $111.48(18)$ & $\mathrm{C}(14)-\mathrm{C}(13)-\mathrm{C}(12)$ & $118.1(2)$ \\
\hline $\mathrm{C}(1)-\mathrm{C}(2)-\mathrm{H}(2 \mathrm{~A})$ & 109.3 & $\mathrm{C}(14)-\mathrm{C}(13)-\mathrm{C}(39)$ & $122.8(2)$ \\
\hline $\mathrm{P}(1)-\mathrm{C}(2)-\mathrm{H}(2 \mathrm{~A})$ & 109.3 & $\mathrm{C}(12)-\mathrm{C}(13)-\mathrm{C}(39)$ & $119.0(2)$ \\
\hline $\mathrm{C}(1)-\mathrm{C}(2)-\mathrm{H}(2 \mathrm{~B})$ & 109.3 & $\mathrm{C}(13)-\mathrm{C}(14)-\mathrm{C}(9)$ & $120.2(2)$ \\
\hline $\mathrm{P}(1)-\mathrm{C}(2)-\mathrm{H}(2 \mathrm{~B})$ & 109.3 & $\mathrm{C}(13)-\mathrm{C}(14)-\mathrm{H}(14)$ & 119.9 \\
\hline $\mathrm{H}(2 \mathrm{~A})-\mathrm{C}(2)-\mathrm{H}(2 \mathrm{~B})$ & 108.0 & $\mathrm{C}(9)-\mathrm{C}(14)-\mathrm{H}(14)$ & 119.9 \\
\hline$C(8)-C(3)-C(4)$ & $120.0(2)$ & $C(16)-C(15)-C(20)$ & $119.3(2)$ \\
\hline $\mathrm{C}(8)-\mathrm{C}(3)-\mathrm{P}(1)$ & $119.4(2)$ & $\mathrm{C}(16)-\mathrm{C}(15)-\mathrm{P}(2)$ & $116.5(2)$ \\
\hline $\mathrm{C}(4)-\mathrm{C}(3)-\mathrm{P}(1)$ & $120.6(2)$ & $\mathrm{C}(20)-\mathrm{C}(15)-\mathrm{P}(2)$ & $124.0(2)$ \\
\hline$C(5)-C(4)-C(3)$ & $121.1(2)$ & $\mathrm{C}(15)-\mathrm{C}(16)-\mathrm{C}(17)$ & $121.6(3)$ \\
\hline $\mathrm{C}(5)-\mathrm{C}(4)-\mathrm{H}(4)$ & 119.5 & $\mathrm{C}(15)-\mathrm{C}(16)-\mathrm{H}(16)$ & 119.2 \\
\hline $\mathrm{C}(3)-\mathrm{C}(4)-\mathrm{H}(4)$ & 119.5 & $\mathrm{C}(17)-\mathrm{C}(16)-\mathrm{H}(16)$ & 119.2 \\
\hline$C(4)-C(5)-C(6)$ & $117.9(2)$ & $\mathrm{C}(16)-\mathrm{C}(17)-\mathrm{C}(18)$ & $117.4(2)$ \\
\hline $\mathrm{C}(4)-\mathrm{C}(5)-\mathrm{C}(31)$ & $119.0(2)$ & $\mathrm{C}(16)-\mathrm{C}(17)-\mathrm{C}(55)$ & $118.0(3)$ \\
\hline$C(6)-C(5)-C(31)$ & $123.1(2)$ & $\mathrm{C}(18)-\mathrm{C}(17)-\mathrm{C}(55)$ & $124.6(3)$ \\
\hline$C(7)-C(6)-C(5)$ & $122.0(2)$ & $\mathrm{C}(16)-\mathrm{C}(17)-\mathrm{C}\left(55^{\prime}\right)$ & $126.7(13)$ \\
\hline $\mathrm{C}(7)-\mathrm{C}(6)-\mathrm{H}(6)$ & 119.0 & $\mathrm{C}(18)-\mathrm{C}(17)-\mathrm{C}\left(55^{\prime}\right)$ & $113.5(15)$ \\
\hline $\mathrm{C}(5)-\mathrm{C}(6)-\mathrm{H}(6)$ & 119.0 & $\mathrm{C}(17)-\mathrm{C}(18)-\mathrm{C}(19)$ & $122.8(2)$ \\
\hline $\mathrm{C}(6)-\mathrm{C}(7)-\mathrm{C}(8)$ & $118.8(2)$ & $\mathrm{C}(17)-\mathrm{C}(18)-\mathrm{H}(18)$ & 118.6 \\
\hline$C(6)-C(7)-C(27)$ & $122.4(2)$ & $\mathrm{C}(19)-\mathrm{C}(18)-\mathrm{H}(18)$ & 118.6 \\
\hline$C(8)-C(7)-C(27)$ & $118.8(2)$ & $\mathrm{C}(20)-\mathrm{C}(19)-\mathrm{C}(18)$ & $118.0(2)$ \\
\hline$C(3)-C(8)-C(7)$ & $120.3(2)$ & $\mathrm{C}(20)-\mathrm{C}(19)-\mathrm{C}(51)$ & $122.3(2)$ \\
\hline $\mathrm{C}(3)-\mathrm{C}(8)-\mathrm{H}(8)$ & 119.9 & $\mathrm{C}(18)-\mathrm{C}(19)-\mathrm{C}(51)$ & $119.7(2)$ \\
\hline $\mathrm{C}(7)-\mathrm{C}(8)-\mathrm{H}(8)$ & 119.9 & $\mathrm{C}(19)-\mathrm{C}(20)-\mathrm{C}(15)$ & $120.9(2)$ \\
\hline $\mathrm{C}(10)-\mathrm{C}(9)-\mathrm{C}(14)$ & $119.9(2)$ & $\mathrm{C}(19)-\mathrm{C}(20)-\mathrm{H}(20)$ & 119.5 \\
\hline $\mathrm{C}(10)-\mathrm{C}(9)-\mathrm{P}(1)$ & $115.3(2)$ & $\mathrm{C}(15)-\mathrm{C}(20)-\mathrm{H}(20)$ & 119.5 \\
\hline $\mathrm{C}(14)-\mathrm{C}(9)-\mathrm{P}(1)$ & $124.6(2)$ & $\mathrm{C}(26)-\mathrm{C}(21)-\mathrm{C}(22)$ & $119.4(2)$ \\
\hline $\mathrm{C}(9)-\mathrm{C}(10)-\mathrm{C}(11)$ & $121.5(3)$ & $\mathrm{C}(26)-\mathrm{C}(21)-\mathrm{P}(2)$ & $117.9(2)$ \\
\hline
\end{tabular}




\begin{tabular}{|c|c|}
\hline $\mathrm{C}(22)-\mathrm{C}(21)-\mathrm{P}(2)$ & $122.7(2)$ \\
\hline$C(23)-C(22)-C(21)$ & $120.8(2)$ \\
\hline $\mathrm{C}(23)-\mathrm{C}(22)-\mathrm{H}(22)$ & 119.6 \\
\hline $\mathrm{C}(21)-\mathrm{C}(22)-\mathrm{H}(22)$ & 119.6 \\
\hline $\mathrm{C}(22)-\mathrm{C}(23)-\mathrm{C}(24)$ & $118.3(2)$ \\
\hline $\mathrm{C}(22)-\mathrm{C}(23)-\mathrm{C}(47)$ & $122.0(2)$ \\
\hline $\mathrm{C}(24)-\mathrm{C}(23)-\mathrm{C}(47)$ & $119.7(2)$ \\
\hline$C(25)-C(24)-C(23)$ & $122.1(3)$ \\
\hline $\mathrm{C}(25)-\mathrm{C}(24)-\mathrm{H}(24)$ & 118.9 \\
\hline $\mathrm{C}(23)-\mathrm{C}(24)-\mathrm{H}(24)$ & 118.9 \\
\hline$C(24)-C(25)-C(26)$ & $118.3(2)$ \\
\hline $\mathrm{C}(24)-\mathrm{C}(25)-\mathrm{C}(43)$ & $123.3(2)$ \\
\hline$C(26)-C(25)-C(43)$ & $118.4(2)$ \\
\hline$C(21)-C(26)-C(25)$ & $121.0(2)$ \\
\hline $\mathrm{C}(21)-\mathrm{C}(26)-\mathrm{H}(26)$ & 119.5 \\
\hline $\mathrm{C}(25)-\mathrm{C}(26)-\mathrm{H}(26)$ & 119.5 \\
\hline $\mathrm{C}(28)-\mathrm{C}(27)-\mathrm{C}(29)$ & $109.0(3)$ \\
\hline $\mathrm{C}(28)-\mathrm{C}(27)-\mathrm{C}(30)$ & $107.8(3)$ \\
\hline $\mathrm{C}(29)-\mathrm{C}(27)-\mathrm{C}(30)$ & $108.5(3)$ \\
\hline $\mathrm{C}(28)-\mathrm{C}(27)-\mathrm{C}(7)$ & $112.6(2)$ \\
\hline $\mathrm{C}(29)-\mathrm{C}(27)-\mathrm{C}(7)$ & $109.5(2)$ \\
\hline$C(30)-C(27)-C(7)$ & $109.4(2)$ \\
\hline $\mathrm{C}(27)-\mathrm{C}(28)-\mathrm{H}(28 \mathrm{~A})$ & 109.5 \\
\hline $\mathrm{C}(27)-\mathrm{C}(28)-\mathrm{H}(28 \mathrm{~B})$ & 109.5 \\
\hline $\mathrm{H}(28 \mathrm{~A})-\mathrm{C}(28)-\mathrm{H}(28 \mathrm{~B})$ & 109.5 \\
\hline $\mathrm{C}(27)-\mathrm{C}(28)-\mathrm{H}(28 \mathrm{C})$ & 109.5 \\
\hline $\mathrm{H}(28 \mathrm{~A})-\mathrm{C}(28)-\mathrm{H}(28 \mathrm{C})$ & 109.5 \\
\hline $\mathrm{H}(28 \mathrm{~B})-\mathrm{C}(28)-\mathrm{H}(28 \mathrm{C})$ & 109.5 \\
\hline $\mathrm{C}(27)-\mathrm{C}(29)-\mathrm{H}(29 \mathrm{~A})$ & 109.5 \\
\hline $\mathrm{C}(27)-\mathrm{C}(29)-\mathrm{H}(29 \mathrm{~B})$ & 109.5 \\
\hline H(29A)-C(29)-H(29B) & 109.5 \\
\hline $\mathrm{C}(27)-\mathrm{C}(29)-\mathrm{H}(29 \mathrm{C})$ & 109.5 \\
\hline $\mathrm{H}(29 \mathrm{~A})-\mathrm{C}(29)-\mathrm{H}(29 \mathrm{C})$ & 109.5 \\
\hline H(29B)-C(29)-H(29C) & 109.5 \\
\hline $\mathrm{C}(27)-\mathrm{C}(30)-\mathrm{H}(30 \mathrm{~A})$ & 109.5 \\
\hline $\mathrm{C}(27)-\mathrm{C}(30)-\mathrm{H}(30 \mathrm{~B})$ & 109.5 \\
\hline
\end{tabular}

\begin{tabular}{|c|c|}
\hline $\mathrm{H}(30 \mathrm{~A})-\mathrm{C}(30)-\mathrm{H}(30 \mathrm{~B})$ & 109.5 \\
\hline $\mathrm{C}(27)-\mathrm{C}(30)-\mathrm{H}(30 \mathrm{C})$ & 109.5 \\
\hline $\mathrm{H}(30 \mathrm{~A})-\mathrm{C}(30)-\mathrm{H}(30 \mathrm{C})$ & 109.5 \\
\hline $\mathrm{H}(30 \mathrm{~B})-\mathrm{C}(30)-\mathrm{H}(30 \mathrm{C})$ & 109.5 \\
\hline C(32)-C(31)-C(34) & $108.5(3)$ \\
\hline $\mathrm{C}(32)-\mathrm{C}(31)-\mathrm{C}(5)$ & $112.6(2)$ \\
\hline $\mathrm{C}(34)-\mathrm{C}(31)-\mathrm{C}(5)$ & $108.8(2)$ \\
\hline $\mathrm{C}(32)-\mathrm{C}(31)-\mathrm{C}(33)$ & $107.4(2)$ \\
\hline$C(34)-C(31)-C(33)$ & $109.0(3)$ \\
\hline$C(5)-C(31)-C(33)$ & $110.3(2)$ \\
\hline $\mathrm{C}(31)-\mathrm{C}(32)-\mathrm{H}(32 \mathrm{~A})$ & 109.5 \\
\hline $\mathrm{C}(31)-\mathrm{C}(32)-\mathrm{H}(32 \mathrm{~B})$ & 109.5 \\
\hline $\mathrm{H}(32 \mathrm{~A})-\mathrm{C}(32)-\mathrm{H}(32 \mathrm{~B})$ & 109.5 \\
\hline $\mathrm{C}(31)-\mathrm{C}(32)-\mathrm{H}(32 \mathrm{C})$ & 109.5 \\
\hline $\mathrm{H}(32 \mathrm{~A})-\mathrm{C}(32)-\mathrm{H}(32 \mathrm{C})$ & 109.5 \\
\hline $\mathrm{H}(32 \mathrm{~B})-\mathrm{C}(32)-\mathrm{H}(32 \mathrm{C})$ & 109.5 \\
\hline $\mathrm{C}(31)-\mathrm{C}(33)-\mathrm{H}(33 \mathrm{~A})$ & 109.5 \\
\hline $\mathrm{C}(31)-\mathrm{C}(33)-\mathrm{H}(33 \mathrm{~B})$ & 109.5 \\
\hline $\mathrm{H}(33 \mathrm{~A})-\mathrm{C}(33)-\mathrm{H}(33 \mathrm{~B})$ & 109.5 \\
\hline $\mathrm{C}(31)-\mathrm{C}(33)-\mathrm{H}(33 \mathrm{C})$ & 109.5 \\
\hline $\mathrm{H}(33 \mathrm{~A})-\mathrm{C}(33)-\mathrm{H}(33 \mathrm{C})$ & 109.5 \\
\hline $\mathrm{H}(33 \mathrm{~B})-\mathrm{C}(33)-\mathrm{H}(33 \mathrm{C})$ & 109.5 \\
\hline $\mathrm{C}(31)-\mathrm{C}(34)-\mathrm{H}(34 \mathrm{~A})$ & 109.5 \\
\hline $\mathrm{C}(31)-\mathrm{C}(34)-\mathrm{H}(34 \mathrm{~B})$ & 109.5 \\
\hline $\mathrm{H}(34 \mathrm{~A})-\mathrm{C}(34)-\mathrm{H}(34 \mathrm{~B})$ & 109.5 \\
\hline $\mathrm{C}(31)-\mathrm{C}(34)-\mathrm{H}(34 \mathrm{C})$ & 109.5 \\
\hline $\mathrm{H}(34 \mathrm{~A})-\mathrm{C}(34)-\mathrm{H}(34 \mathrm{C})$ & 109.5 \\
\hline $\mathrm{H}(34 \mathrm{~B})-\mathrm{C}(34)-\mathrm{H}(34 \mathrm{C})$ & 109.5 \\
\hline $\mathrm{C}(38)-\mathrm{C}(35)-\mathrm{C}(11)$ & $109.1(2)$ \\
\hline $\mathrm{C}(38)-\mathrm{C}(35)-\mathrm{C}(37)$ & $109.0(3)$ \\
\hline$C(11)-C(35)-C(37)$ & $110.1(2)$ \\
\hline $\mathrm{C}(38)-\mathrm{C}(35)-\mathrm{C}(36)$ & $108.6(3)$ \\
\hline $\mathrm{C}(11)-\mathrm{C}(35)-\mathrm{C}(36)$ & $112.1(2)$ \\
\hline $\mathrm{C}(37)-\mathrm{C}(35)-\mathrm{C}(36)$ & $108.0(3)$ \\
\hline $\mathrm{C}(35)-\mathrm{C}(36)-\mathrm{H}(36 \mathrm{~A})$ & 109.5 \\
\hline $\mathrm{C}(35)-\mathrm{C}(36)-\mathrm{H}(36 \mathrm{~B})$ & 109.5 \\
\hline
\end{tabular}




\begin{tabular}{|c|c|c|c|}
\hline $\mathrm{H}(36 \mathrm{~A})-\mathrm{C}(36)-\mathrm{H}(36 \mathrm{~B})$ & 109.5 & $\mathrm{H}(42 \mathrm{~A})-\mathrm{C}(42)-\mathrm{H}(42 \mathrm{~B})$ & 109.5 \\
\hline $\mathrm{C}(35)-\mathrm{C}(36)-\mathrm{H}(36 \mathrm{C})$ & 109.5 & $\mathrm{C}(39)-\mathrm{C}(42)-\mathrm{H}(42 \mathrm{C})$ & 109.5 \\
\hline $\mathrm{H}(36 \mathrm{~A})-\mathrm{C}(36)-\mathrm{H}(36 \mathrm{C})$ & 109.5 & $\mathrm{H}(42 \mathrm{~A})-\mathrm{C}(42)-\mathrm{H}(42 \mathrm{C})$ & 109.5 \\
\hline $\mathrm{H}(36 \mathrm{~B})-\mathrm{C}(36)-\mathrm{H}(36 \mathrm{C})$ & 109.5 & $\mathrm{H}(42 \mathrm{~B})-\mathrm{C}(42)-\mathrm{H}(42 \mathrm{C})$ & 109.5 \\
\hline $\mathrm{C}(35)-\mathrm{C}(37)-\mathrm{H}(37 \mathrm{~A})$ & 109.5 & $\mathrm{C}(44)-\mathrm{C}(43)-\mathrm{C}(46)$ & $111.7(3)$ \\
\hline $\mathrm{C}(35)-\mathrm{C}(37)-\mathrm{H}(37 \mathrm{~B})$ & 109.5 & $\mathrm{C}(44)-\mathrm{C}(43)-\mathrm{C}(25)$ & $108.9(2)$ \\
\hline H(37A)-C(37)-H(37B) & 109.5 & $\mathrm{C}(46)-\mathrm{C}(43)-\mathrm{C}(25)$ & $108.8(2)$ \\
\hline $\mathrm{C}(35)-\mathrm{C}(37)-\mathrm{H}(37 \mathrm{C})$ & 109.5 & $\mathrm{C}(44)-\mathrm{C}(43)-\mathrm{C}(45)$ & $108.7(3)$ \\
\hline H(37A)-C(37)-H(37C) & 109.5 & $\mathrm{C}(46)-\mathrm{C}(43)-\mathrm{C}(45)$ & $107.0(3)$ \\
\hline H(37B)-C(37)-H(37C) & 109.5 & $\mathrm{C}(25)-\mathrm{C}(43)-\mathrm{C}(45)$ & $111.7(2)$ \\
\hline $\mathrm{C}(35)-\mathrm{C}(38)-\mathrm{H}(38 \mathrm{~A})$ & 109.5 & $\mathrm{C}(43)-\mathrm{C}(44)-\mathrm{H}(44 \mathrm{~A})$ & 109.5 \\
\hline $\mathrm{C}(35)-\mathrm{C}(38)-\mathrm{H}(38 \mathrm{~B})$ & 109.5 & $\mathrm{C}(43)-\mathrm{C}(44)-\mathrm{H}(44 \mathrm{~B})$ & 109.5 \\
\hline $\mathrm{H}(38 \mathrm{~A})-\mathrm{C}(38)-\mathrm{H}(38 \mathrm{~B})$ & 109.5 & $\mathrm{H}(44 \mathrm{~A})-\mathrm{C}(44)-\mathrm{H}(44 \mathrm{~B})$ & 109.5 \\
\hline $\mathrm{C}(35)-\mathrm{C}(38)-\mathrm{H}(38 \mathrm{C})$ & 109.5 & $\mathrm{C}(43)-\mathrm{C}(44)-\mathrm{H}(44 \mathrm{C})$ & 109.5 \\
\hline $\mathrm{H}(38 \mathrm{~A})-\mathrm{C}(38)-\mathrm{H}(38 \mathrm{C})$ & 109.5 & $\mathrm{H}(44 \mathrm{~A})-\mathrm{C}(44)-\mathrm{H}(44 \mathrm{C})$ & 109.5 \\
\hline $\mathrm{H}(38 \mathrm{~B})-\mathrm{C}(38)-\mathrm{H}(38 \mathrm{C})$ & 109.5 & $\mathrm{H}(44 \mathrm{~B})-\mathrm{C}(44)-\mathrm{H}(44 \mathrm{C})$ & 109.5 \\
\hline C(40)-C(39)-C(13) & $112.2(2)$ & $\mathrm{C}(43)-\mathrm{C}(45)-\mathrm{H}(45 \mathrm{~A})$ & 109.5 \\
\hline C(40)-C(39)-C(41) & $108.1(2)$ & $\mathrm{C}(43)-\mathrm{C}(45)-\mathrm{H}(45 \mathrm{~B})$ & 109.5 \\
\hline C(13)-C(39)-C(41) & $109.1(2)$ & $\mathrm{H}(45 \mathrm{~A})-\mathrm{C}(45)-\mathrm{H}(45 \mathrm{~B})$ & 109.5 \\
\hline $\mathrm{C}(40)-\mathrm{C}(39)-\mathrm{C}(42)$ & $108.7(3)$ & $\mathrm{C}(43)-\mathrm{C}(45)-\mathrm{H}(45 \mathrm{C})$ & 109.5 \\
\hline C(13)-C(39)-C(42) & $109.6(2)$ & $\mathrm{H}(45 \mathrm{~A})-\mathrm{C}(45)-\mathrm{H}(45 \mathrm{C})$ & 109.5 \\
\hline C(41)-C(39)-C(42) & $109.1(2)$ & $\mathrm{H}(45 \mathrm{~B})-\mathrm{C}(45)-\mathrm{H}(45 \mathrm{C})$ & 109.5 \\
\hline $\mathrm{C}(39)-\mathrm{C}(40)-\mathrm{H}(40 \mathrm{~A})$ & 109.5 & $\mathrm{C}(43)-\mathrm{C}(46)-\mathrm{H}(46 \mathrm{~A})$ & 109.5 \\
\hline $\mathrm{C}(39)-\mathrm{C}(40)-\mathrm{H}(40 \mathrm{~B})$ & 109.5 & $\mathrm{C}(43)-\mathrm{C}(46)-\mathrm{H}(46 \mathrm{~B})$ & 109.5 \\
\hline $\mathrm{H}(40 \mathrm{~A})-\mathrm{C}(40)-\mathrm{H}(40 \mathrm{~B})$ & 109.5 & $\mathrm{H}(46 \mathrm{~A})-\mathrm{C}(46)-\mathrm{H}(46 \mathrm{~B})$ & 109.5 \\
\hline $\mathrm{C}(39)-\mathrm{C}(40)-\mathrm{H}(40 \mathrm{C})$ & 109.5 & $\mathrm{C}(43)-\mathrm{C}(46)-\mathrm{H}(46 \mathrm{C})$ & 109.5 \\
\hline $\mathrm{H}(40 \mathrm{~A})-\mathrm{C}(40)-\mathrm{H}(40 \mathrm{C})$ & 109.5 & $\mathrm{H}(46 \mathrm{~A})-\mathrm{C}(46)-\mathrm{H}(46 \mathrm{C})$ & 109.5 \\
\hline $\mathrm{H}(40 \mathrm{~B})-\mathrm{C}(40)-\mathrm{H}(40 \mathrm{C})$ & 109.5 & $\mathrm{H}(46 \mathrm{~B})-\mathrm{C}(46)-\mathrm{H}(46 \mathrm{C})$ & 109.5 \\
\hline $\mathrm{C}(39)-\mathrm{C}(41)-\mathrm{H}(41 \mathrm{~A})$ & 109.5 & $\mathrm{C}(50)-\mathrm{C}(47)-\mathrm{C}(48)$ & $109.0(3)$ \\
\hline $\mathrm{C}(39)-\mathrm{C}(41)-\mathrm{H}(41 \mathrm{~B})$ & 109.5 & $\mathrm{C}(50)-\mathrm{C}(47)-\mathrm{C}(49)$ & $107.7(3)$ \\
\hline $\mathrm{H}(41 \mathrm{~A})-\mathrm{C}(41)-\mathrm{H}(41 \mathrm{~B})$ & 109.5 & $\mathrm{C}(48)-\mathrm{C}(47)-\mathrm{C}(49)$ & $109.1(3)$ \\
\hline C(39)-C(41)-H(41C) & 109.5 & $\mathrm{C}(50)-\mathrm{C}(47)-\mathrm{C}(23)$ & $111.8(2)$ \\
\hline $\mathrm{H}(41 \mathrm{~A})-\mathrm{C}(41)-\mathrm{H}(41 \mathrm{C})$ & 109.5 & $\mathrm{C}(48)-\mathrm{C}(47)-\mathrm{C}(23)$ & $109.1(2)$ \\
\hline $\mathrm{H}(41 \mathrm{~B})-\mathrm{C}(41)-\mathrm{H}(41 \mathrm{C})$ & 109.5 & $\mathrm{C}(49)-\mathrm{C}(47)-\mathrm{C}(23)$ & $110.0(2)$ \\
\hline C(39)-C(42)-H(42A) & 109.5 & $\mathrm{C}(47)-\mathrm{C}(48)-\mathrm{H}(48 \mathrm{~A})$ & 109.5 \\
\hline C(39)-C(42)-H(42B) & 109.5 & $\mathrm{C}(47)-\mathrm{C}(48)-\mathrm{H}(48 \mathrm{~B})$ & 109.5 \\
\hline
\end{tabular}




\begin{tabular}{|c|c|c|c|}
\hline $\mathrm{H}(48 \mathrm{~A})-\mathrm{C}(48)-\mathrm{H}(48 \mathrm{~B})$ & 109.5 & $\mathrm{H}(54 \mathrm{~A})-\mathrm{C}(54)-\mathrm{H}(54 \mathrm{~B})$ & 109.5 \\
\hline $\mathrm{C}(47)-\mathrm{C}(48)-\mathrm{H}(48 \mathrm{C})$ & 109.5 & $\mathrm{C}(51)-\mathrm{C}(54)-\mathrm{H}(54 \mathrm{C})$ & 109.5 \\
\hline $\mathrm{H}(48 \mathrm{~A})-\mathrm{C}(48)-\mathrm{H}(48 \mathrm{C})$ & 109.5 & $\mathrm{H}(54 \mathrm{~A})-\mathrm{C}(54)-\mathrm{H}(54 \mathrm{C})$ & 109.5 \\
\hline $\mathrm{H}(48 \mathrm{~B})-\mathrm{C}(48)-\mathrm{H}(48 \mathrm{C})$ & 109.5 & $\mathrm{H}(54 \mathrm{~B})-\mathrm{C}(54)-\mathrm{H}(54 \mathrm{C})$ & 109.5 \\
\hline $\mathrm{C}(47)-\mathrm{C}(49)-\mathrm{H}(49 \mathrm{~A})$ & 109.5 & $\mathrm{C}(57)-\mathrm{C}(55)-\mathrm{C}(58)$ & $108.2(3)$ \\
\hline $\mathrm{C}(47)-\mathrm{C}(49)-\mathrm{H}(49 \mathrm{~B})$ & 109.5 & $\mathrm{C}(57)-\mathrm{C}(55)-\mathrm{C}(17)$ & $110.6(3)$ \\
\hline $\mathrm{H}(49 \mathrm{~A})-\mathrm{C}(49)-\mathrm{H}(49 \mathrm{~B})$ & 109.5 & $\mathrm{C}(58)-\mathrm{C}(55)-\mathrm{C}(17)$ & $111.9(3)$ \\
\hline $\mathrm{C}(47)-\mathrm{C}(49)-\mathrm{H}(49 \mathrm{C})$ & 109.5 & $C(57)-C(55)-C(56)$ & $110.5(4)$ \\
\hline $\mathrm{H}(49 \mathrm{~A})-\mathrm{C}(49)-\mathrm{H}(49 \mathrm{C})$ & 109.5 & $\mathrm{C}(58)-\mathrm{C}(55)-\mathrm{C}(56)$ & $108.0(3)$ \\
\hline H(49B)-C(49)-H(49C) & 109.5 & $C(17)-C(55)-C(56)$ & $107.6(3)$ \\
\hline $\mathrm{C}(47)-\mathrm{C}(50)-\mathrm{H}(50 \mathrm{~A})$ & 109.5 & $\mathrm{C}(55)-\mathrm{C}(56)-\mathrm{H}(56 \mathrm{~A})$ & 109.5 \\
\hline $\mathrm{C}(47)-\mathrm{C}(50)-\mathrm{H}(50 \mathrm{~B})$ & 109.5 & $\mathrm{C}(55)-\mathrm{C}(56)-\mathrm{H}(56 \mathrm{~B})$ & 109.5 \\
\hline $\mathrm{H}(50 \mathrm{~A})-\mathrm{C}(50)-\mathrm{H}(50 \mathrm{~B})$ & 109.5 & $\mathrm{H}(56 \mathrm{~A})-\mathrm{C}(56)-\mathrm{H}(56 \mathrm{~B})$ & 109.5 \\
\hline $\mathrm{C}(47)-\mathrm{C}(50)-\mathrm{H}(50 \mathrm{C})$ & 109.5 & $\mathrm{C}(55)-\mathrm{C}(56)-\mathrm{H}(56 \mathrm{C})$ & 109.5 \\
\hline $\mathrm{H}(50 \mathrm{~A})-\mathrm{C}(50)-\mathrm{H}(50 \mathrm{C})$ & 109.5 & $\mathrm{H}(56 \mathrm{~A})-\mathrm{C}(56)-\mathrm{H}(56 \mathrm{C})$ & 109.5 \\
\hline $\mathrm{H}(50 \mathrm{~B})-\mathrm{C}(50)-\mathrm{H}(50 \mathrm{C})$ & 109.5 & $\mathrm{H}(56 \mathrm{~B})-\mathrm{C}(56)-\mathrm{H}(56 \mathrm{C})$ & 109.5 \\
\hline $\mathrm{C}(53)-\mathrm{C}(51)-\mathrm{C}(52)$ & $108.6(2)$ & $\mathrm{C}(55)-\mathrm{C}(57)-\mathrm{H}(57 \mathrm{~A})$ & 109.5 \\
\hline $\mathrm{C}(53)-\mathrm{C}(51)-\mathrm{C}(19)$ & $112.1(2)$ & $\mathrm{C}(55)-\mathrm{C}(57)-\mathrm{H}(57 \mathrm{~B})$ & 109.5 \\
\hline$C(52)-C(51)-C(19)$ & $109.2(2)$ & $\mathrm{H}(57 \mathrm{~A})-\mathrm{C}(57)-\mathrm{H}(57 \mathrm{~B})$ & 109.5 \\
\hline$C(53)-C(51)-C(54)$ & $107.9(2)$ & $\mathrm{C}(55)-\mathrm{C}(57)-\mathrm{H}(57 \mathrm{C})$ & 109.5 \\
\hline$C(52)-C(51)-C(54)$ & $109.7(2)$ & $\mathrm{H}(57 \mathrm{~A})-\mathrm{C}(57)-\mathrm{H}(57 \mathrm{C})$ & 109.5 \\
\hline$C(19)-C(51)-C(54)$ & $109.3(2)$ & $\mathrm{H}(57 \mathrm{~B})-\mathrm{C}(57)-\mathrm{H}(57 \mathrm{C})$ & 109.5 \\
\hline $\mathrm{C}(51)-\mathrm{C}(52)-\mathrm{H}(52 \mathrm{~A})$ & 109.5 & $\mathrm{C}(55)-\mathrm{C}(58)-\mathrm{H}(58 \mathrm{~A})$ & 109.5 \\
\hline $\mathrm{C}(51)-\mathrm{C}(52)-\mathrm{H}(52 \mathrm{~B})$ & 109.5 & $\mathrm{C}(55)-\mathrm{C}(58)-\mathrm{H}(58 \mathrm{~B})$ & 109.5 \\
\hline $\mathrm{H}(52 \mathrm{~A})-\mathrm{C}(52)-\mathrm{H}(52 \mathrm{~B})$ & 109.5 & $\mathrm{H}(58 \mathrm{~A})-\mathrm{C}(58)-\mathrm{H}(58 \mathrm{~B})$ & 109.5 \\
\hline $\mathrm{C}(51)-\mathrm{C}(52)-\mathrm{H}(52 \mathrm{C})$ & 109.5 & $\mathrm{C}(55)-\mathrm{C}(58)-\mathrm{H}(58 \mathrm{C})$ & 109.5 \\
\hline $\mathrm{H}(52 \mathrm{~A})-\mathrm{C}(52)-\mathrm{H}(52 \mathrm{C})$ & 109.5 & $\mathrm{H}(58 \mathrm{~A})-\mathrm{C}(58)-\mathrm{H}(58 \mathrm{C})$ & 109.5 \\
\hline $\mathrm{H}(52 \mathrm{~B})-\mathrm{C}(52)-\mathrm{H}(52 \mathrm{C})$ & 109.5 & $\mathrm{H}(58 \mathrm{~B})-\mathrm{C}(58)-\mathrm{H}(58 \mathrm{C})$ & 109.5 \\
\hline $\mathrm{C}(51)-\mathrm{C}(53)-\mathrm{H}(53 \mathrm{~A})$ & 109.5 & $\mathrm{C}\left(57^{\prime}\right)-\mathrm{C}\left(55^{\prime}\right)-\mathrm{C}\left(58^{\prime}\right)$ & $109(2)$ \\
\hline $\mathrm{C}(51)-\mathrm{C}(53)-\mathrm{H}(53 \mathrm{~B})$ & 109.5 & $C\left(57^{\prime}\right)-C\left(55^{\prime}\right)-C(17)$ & $103(3)$ \\
\hline $\mathrm{H}(53 \mathrm{~A})-\mathrm{C}(53)-\mathrm{H}(53 \mathrm{~B})$ & 109.5 & $\mathrm{C}\left(58^{\prime}\right)-\mathrm{C}\left(55^{\prime}\right)-\mathrm{C}(17)$ & $116(3)$ \\
\hline $\mathrm{C}(51)-\mathrm{C}(53)-\mathrm{H}(53 \mathrm{C})$ & 109.5 & $\mathrm{C}\left(57^{\prime}\right)-\mathrm{C}\left(55^{\prime}\right)-\mathrm{C}\left(56^{\prime}\right)$ & $110(2)$ \\
\hline $\mathrm{H}(53 \mathrm{~A})-\mathrm{C}(53)-\mathrm{H}(53 \mathrm{C})$ & 109.5 & $\mathrm{C}\left(58^{\prime}\right)-\mathrm{C}\left(55^{\prime}\right)-\mathrm{C}\left(56^{\prime}\right)$ & $106(2)$ \\
\hline $\mathrm{H}(53 \mathrm{~B})-\mathrm{C}(53)-\mathrm{H}(53 \mathrm{C})$ & 109.5 & $\mathrm{C}(17)-\mathrm{C}\left(55^{\prime}\right)-\mathrm{C}\left(56^{\prime}\right)$ & $113(2)$ \\
\hline $\mathrm{C}(51)-\mathrm{C}(54)-\mathrm{H}(54 \mathrm{~A})$ & 109.5 & $\mathrm{C}\left(55^{\prime}\right)-\mathrm{C}\left(56^{\prime}\right)-\mathrm{H}(56 \mathrm{D})$ & 109.5 \\
\hline $\mathrm{C}(51)-\mathrm{C}(54)-\mathrm{H}(54 \mathrm{~B})$ & 109.5 & $\mathrm{C}\left(55^{\prime}\right)-\mathrm{C}\left(56^{\prime}\right)-\mathrm{H}(56 \mathrm{E})$ & 109.5 \\
\hline
\end{tabular}




\begin{tabular}{|c|c|c|c|}
\hline $\mathrm{H}(56 \mathrm{D})-\mathrm{C}\left(56^{\prime}\right)-\mathrm{H}(56 \mathrm{E})$ & 109.5 & $\mathrm{C}(62)-\mathrm{C}(61)-\mathrm{H}(61 \mathrm{~B})$ & 109.2 \\
\hline $\mathrm{C}\left(55^{\prime}\right)-\mathrm{C}\left(56^{\prime}\right)-\mathrm{H}(56 \mathrm{~F})$ & 109.5 & $\mathrm{H}(61 \mathrm{~A})-\mathrm{C}(61)-\mathrm{H}(61 \mathrm{~B})$ & 107.9 \\
\hline $\mathrm{H}(56 \mathrm{D})-\mathrm{C}\left(56^{\prime}\right)-\mathrm{H}(56 \mathrm{~F})$ & 109.5 & $\mathrm{O}(1)-\mathrm{C}(62)-\mathrm{C}(61)$ & $110.1(3)$ \\
\hline $\mathrm{H}(56 \mathrm{E})-\mathrm{C}\left(56^{\prime}\right)-\mathrm{H}(56 \mathrm{~F})$ & 109.5 & $\mathrm{O}(1)-\mathrm{C}(62)-\mathrm{H}(62 \mathrm{~A})$ & 109.6 \\
\hline $\mathrm{C}\left(55^{\prime}\right)-\mathrm{C}\left(57^{\prime}\right)-\mathrm{H}(57 \mathrm{D})$ & 109.5 & $\mathrm{C}(61)-\mathrm{C}(62)-\mathrm{H}(62 \mathrm{~A})$ & 109.6 \\
\hline $\mathrm{C}\left(55^{\prime}\right)-\mathrm{C}\left(57^{\prime}\right)-\mathrm{H}(57 \mathrm{E})$ & 109.5 & $\mathrm{O}(1)-\mathrm{C}(62)-\mathrm{H}(62 \mathrm{~B})$ & 109.6 \\
\hline H(57D)-C(57')-H(57E) & 109.5 & $\mathrm{C}(61)-\mathrm{C}(62)-\mathrm{H}(62 \mathrm{~B})$ & 109.6 \\
\hline $\mathrm{C}\left(55^{\prime}\right)-\mathrm{C}\left(57^{\prime}\right)-\mathrm{H}(57 \mathrm{~F})$ & 109.5 & $\mathrm{H}(62 \mathrm{~A})-\mathrm{C}(62)-\mathrm{H}(62 \mathrm{~B})$ & 108.2 \\
\hline H(57D)-C(57')-H(57F) & 109.5 & $\mathrm{C}(63)-\mathrm{O}(3)-\mathrm{C}(66)$ & $108.8(2)$ \\
\hline $\mathrm{H}(57 \mathrm{E})-\mathrm{C}\left(57^{\prime}\right)-\mathrm{H}(57 \mathrm{~F})$ & 109.5 & $\mathrm{O}(3)-\mathrm{C}(63)-\mathrm{C}(64)$ & $110.9(3)$ \\
\hline $\mathrm{C}\left(55^{\prime}\right)-\mathrm{C}\left(58^{\prime}\right)-\mathrm{H}(58 \mathrm{D})$ & 109.5 & $\mathrm{O}(3)-\mathrm{C}(63)-\mathrm{H}(63 \mathrm{~A})$ & 109.5 \\
\hline $\mathrm{C}\left(55^{\prime}\right)-\mathrm{C}\left(58^{\prime}\right)-\mathrm{H}(58 \mathrm{E})$ & 109.5 & $\mathrm{C}(64)-\mathrm{C}(63)-\mathrm{H}(63 \mathrm{~A})$ & 109.5 \\
\hline $\mathrm{H}(58 \mathrm{D})-\mathrm{C}\left(58^{\prime}\right)-\mathrm{H}(58 \mathrm{E})$ & 109.5 & $\mathrm{O}(3)-\mathrm{C}(63)-\mathrm{H}(63 \mathrm{~B})$ & 109.5 \\
\hline $\mathrm{C}\left(55^{\prime}\right)-\mathrm{C}\left(58^{\prime}\right)-\mathrm{H}(58 \mathrm{~F})$ & 109.5 & $C(64)-C(63)-H(63 B)$ & 109.5 \\
\hline $\mathrm{H}(58 \mathrm{D})-\mathrm{C}\left(58^{\prime}\right)-\mathrm{H}(58 \mathrm{~F})$ & 109.5 & $\mathrm{H}(63 \mathrm{~A})-\mathrm{C}(63)-\mathrm{H}(63 \mathrm{~B})$ & 108.1 \\
\hline $\mathrm{H}(58 \mathrm{E})-\mathrm{C}\left(58^{\prime}\right)-\mathrm{H}(58 \mathrm{~F})$ & 109.5 & $\mathrm{O}(4)-\mathrm{C}(64)-\mathrm{C}(63)$ & $111.2(3)$ \\
\hline $\mathrm{C}(62)-\mathrm{O}(1)-\mathrm{C}(59)$ & $108.9(3)$ & $\mathrm{O}(4)-\mathrm{C}(64)-\mathrm{H}(64 \mathrm{~A})$ & 109.4 \\
\hline $\mathrm{O}(1)-\mathrm{C}(59)-\mathrm{C}(60)$ & $110.3(3)$ & $\mathrm{C}(63)-\mathrm{C}(64)-\mathrm{H}(64 \mathrm{~A})$ & 109.4 \\
\hline $\mathrm{O}(1)-\mathrm{C}(59)-\mathrm{H}(59 \mathrm{~A})$ & 109.6 & $\mathrm{O}(4)-\mathrm{C}(64)-\mathrm{H}(64 \mathrm{~B})$ & 109.4 \\
\hline $\mathrm{C}(60)-\mathrm{C}(59)-\mathrm{H}(59 \mathrm{~A})$ & 109.6 & $\mathrm{C}(63)-\mathrm{C}(64)-\mathrm{H}(64 \mathrm{~B})$ & 109.4 \\
\hline $\mathrm{O}(1)-\mathrm{C}(59)-\mathrm{H}(59 \mathrm{~B})$ & 109.6 & $\mathrm{H}(64 \mathrm{~A})-\mathrm{C}(64)-\mathrm{H}(64 \mathrm{~B})$ & 108.0 \\
\hline $\mathrm{C}(60)-\mathrm{C}(59)-\mathrm{H}(59 \mathrm{~B})$ & 109.6 & $\mathrm{C}(65)-\mathrm{O}(4)-\mathrm{C}(64)$ & $109.3(2)$ \\
\hline $\mathrm{H}(59 \mathrm{~A})-\mathrm{C}(59)-\mathrm{H}(59 \mathrm{~B})$ & 108.1 & $\mathrm{O}(4)-\mathrm{C}(65)-\mathrm{C}(66)$ & $110.9(3)$ \\
\hline $\mathrm{O}(2)-\mathrm{C}(60)-\mathrm{C}(59)$ & $111.3(3)$ & $\mathrm{O}(4)-\mathrm{C}(65)-\mathrm{H}(65 \mathrm{~A})$ & 109.5 \\
\hline $\mathrm{O}(2)-\mathrm{C}(60)-\mathrm{H}(60 \mathrm{~A})$ & 109.4 & $\mathrm{C}(66)-\mathrm{C}(65)-\mathrm{H}(65 \mathrm{~A})$ & 109.5 \\
\hline $\mathrm{C}(59)-\mathrm{C}(60)-\mathrm{H}(60 \mathrm{~A})$ & 109.4 & $\mathrm{O}(4)-\mathrm{C}(65)-\mathrm{H}(65 \mathrm{~B})$ & 109.5 \\
\hline $\mathrm{O}(2)-\mathrm{C}(60)-\mathrm{H}(60 \mathrm{~B})$ & 109.4 & $\mathrm{C}(66)-\mathrm{C}(65)-\mathrm{H}(65 \mathrm{~B})$ & 109.5 \\
\hline $\mathrm{C}(59)-\mathrm{C}(60)-\mathrm{H}(60 \mathrm{~B})$ & 109.4 & $\mathrm{H}(65 \mathrm{~A})-\mathrm{C}(65)-\mathrm{H}(65 \mathrm{~B})$ & 108.0 \\
\hline $\mathrm{H}(60 \mathrm{~A})-\mathrm{C}(60)-\mathrm{H}(60 \mathrm{~B})$ & 108.0 & $\mathrm{O}(3)-\mathrm{C}(66)-\mathrm{C}(65)$ & $110.5(3)$ \\
\hline $\mathrm{C}(61)-\mathrm{O}(2)-\mathrm{C}(60)$ & $108.9(3)$ & $\mathrm{O}(3)-\mathrm{C}(66)-\mathrm{H}(66 \mathrm{~A})$ & 109.5 \\
\hline $\mathrm{O}(2)-\mathrm{C}(61)-\mathrm{C}(62)$ & $111.9(3)$ & $C(65)-C(66)-H(66 A)$ & 109.5 \\
\hline $\mathrm{O}(2)-\mathrm{C}(61)-\mathrm{H}(61 \mathrm{~A})$ & 109.2 & $\mathrm{O}(3)-\mathrm{C}(66)-\mathrm{H}(66 \mathrm{~B})$ & 109.5 \\
\hline $\mathrm{C}(62)-\mathrm{C}(61)-\mathrm{H}(61 \mathrm{~A})$ & 109.2 & $\mathrm{C}(65)-\mathrm{C}(66)-\mathrm{H}(66 \mathrm{~B})$ & 109.5 \\
\hline $\mathrm{O}(2)-\mathrm{C}(61)-\mathrm{H}(61 \mathrm{~B})$ & 109.2 & $\mathrm{H}(66 \mathrm{~A})-\mathrm{C}(66)-\mathrm{H}(66 \mathrm{~B})$ & 108.1 \\
\hline
\end{tabular}


Table S10. Anisotropic displacement parameters $\left(\AA^{2} \times 10^{3}\right)$ for neijk04. The anisotropic displacement factor exponent takes the form: $-2 \pi^{2}\left[h^{2} a^{* 2} U_{11}+\ldots+2 h k a^{*} b^{*} U_{12}\right]$

\begin{tabular}{|c|c|c|c|c|c|c|}
\hline & $\mathrm{U}_{11}$ & $\mathrm{U}_{22}$ & $\mathrm{U}_{33}$ & $\mathrm{U}_{23}$ & $\mathrm{U}_{13}$ & $\mathrm{U}_{12}$ \\
\hline Fe1 & $23(1)$ & 21(1) & $19(1)$ & $6(1)$ & $10(1)$ & $5(1)$ \\
\hline Cl1 & $24(1)$ & $39(1)$ & $40(1)$ & 15(1) & $14(1)$ & $6(1)$ \\
\hline $\mathrm{Cl} 2$ & $48(1)$ & $35(1)$ & $17(1)$ & $0(1)$ & $7(1)$ & $10(1)$ \\
\hline P1 & $18(1)$ & $14(1)$ & $17(1)$ & $1(1)$ & $1(1)$ & $0(1)$ \\
\hline $\mathrm{P} 2$ & 21(1) & $14(1)$ & $14(1)$ & $2(1)$ & $7(1)$ & 1(1) \\
\hline $\mathrm{C} 1$ & $25(1)$ & 19(1) & $16(1)$ & $-1(1)$ & $9(1)$ & $-1(1)$ \\
\hline $\mathrm{C} 2$ & $22(1)$ & 19(1) & $15(1)$ & $1(1)$ & $3(1)$ & $0(1)$ \\
\hline $\mathrm{C} 3$ & $21(1)$ & 19(1) & $17(1)$ & $-1(1)$ & $6(1)$ & $-3(1)$ \\
\hline $\mathrm{C} 4$ & $20(1)$ & 21(1) & $17(1)$ & 1(1) & $4(1)$ & $1(1)$ \\
\hline $\mathrm{C} 5$ & $21(1)$ & $18(1)$ & $18(1)$ & $1(1)$ & $5(1)$ & $2(1)$ \\
\hline C6 & $23(1)$ & 21(1) & $18(1)$ & $-2(1)$ & $7(1)$ & $-4(1)$ \\
\hline $\mathrm{C} 7$ & $19(1)$ & 23(1) & $18(1)$ & $2(1)$ & $4(1)$ & $0(1)$ \\
\hline $\mathrm{C} 8$ & $16(1)$ & 19(1) & $17(1)$ & $3(1)$ & $0(1)$ & $2(1)$ \\
\hline C9 & $17(1)$ & $15(1)$ & $18(1)$ & $0(1)$ & $0(1)$ & $-2(1)$ \\
\hline $\mathrm{C} 10$ & $22(1)$ & 19(1) & $18(1)$ & 1(1) & $6(1)$ & $1(1)$ \\
\hline $\mathrm{C} 11$ & $25(1)$ & 19(1) & $14(1)$ & $-1(1)$ & $1(1)$ & $0(1)$ \\
\hline $\mathrm{C} 12$ & $17(1)$ & 23(1) & $21(2)$ & $-2(1)$ & $0(1)$ & $2(1)$ \\
\hline $\mathrm{C} 13$ & $20(1)$ & 19(1) & $19(1)$ & $0(1)$ & $4(1)$ & $-2(1)$ \\
\hline $\mathrm{C} 14$ & $20(1)$ & $17(1)$ & $14(1)$ & $2(1)$ & $3(1)$ & $-4(1)$ \\
\hline $\mathrm{C} 15$ & $18(1)$ & $16(1)$ & $16(1)$ & $1(1)$ & $4(1)$ & $-3(1)$ \\
\hline $\mathrm{C} 16$ & $26(1)$ & 21(1) & $15(1)$ & $5(1)$ & $6(1)$ & $1(1)$ \\
\hline $\mathrm{C} 17$ & $23(1)$ & $28(2)$ & $21(2)$ & $3(1)$ & $9(1)$ & $3(1)$ \\
\hline $\mathrm{C} 18$ & 21(1) & 21(1) & $19(1)$ & $3(1)$ & $3(1)$ & $0(1)$ \\
\hline C19 & $23(1)$ & $17(1)$ & $16(1)$ & $0(1)$ & $4(1)$ & $-4(1)$ \\
\hline $\mathrm{C} 20$ & $22(1)$ & $15(1)$ & $17(1)$ & $-2(1)$ & $5(1)$ & $-3(1)$ \\
\hline $\mathrm{C} 21$ & $22(1)$ & $18(1)$ & $12(1)$ & $-1(1)$ & $7(1)$ & 2(1) \\
\hline $\mathrm{C} 22$ & 21(1) & $18(1)$ & $14(1)$ & $2(1)$ & $7(1)$ & $5(1)$ \\
\hline $\mathrm{C} 23$ & $27(1)$ & 19(1) & $14(1)$ & $-1(1)$ & $10(1)$ & $0(1)$ \\
\hline $\mathrm{C} 24$ & $22(1)$ & $25(1)$ & $17(1)$ & $-4(1)$ & $7(1)$ & $-2(1)$ \\
\hline $\mathrm{C} 25$ & $20(1)$ & $28(2)$ & 11(1) & $-5(1)$ & $5(1)$ & $4(1)$ \\
\hline C26 & $27(1)$ & $20(1)$ & $11(1)$ & $1(1)$ & $6(1)$ & $6(1)$ \\
\hline
\end{tabular}




\begin{tabular}{|c|c|c|c|c|c|c|}
\hline $\mathrm{C} 27$ & $18(1)$ & 21(1) & $25(2)$ & $5(1)$ & $-1(1)$ & $-3(1)$ \\
\hline $\mathrm{C} 28$ & $34(2)$ & $37(2)$ & $111(4)$ & $23(2)$ & $-26(2)$ & $-14(2)$ \\
\hline $\mathrm{C} 29$ & $29(2)$ & $73(3)$ & $24(2)$ & $-6(2)$ & $-1(1)$ & $3(2)$ \\
\hline $\mathrm{C} 30$ & $19(1)$ & $59(2)$ & $31(2)$ & $-2(2)$ & $1(1)$ & $5(1)$ \\
\hline C31 & $22(1)$ & $19(1)$ & $26(2)$ & $0(1)$ & $3(1)$ & $2(1)$ \\
\hline C32 & $34(2)$ & $20(2)$ & $42(2)$ & $6(1)$ & $-4(2)$ & $0(1)$ \\
\hline C33 & $45(2)$ & $24(2)$ & $22(2)$ & $3(1)$ & $-4(1)$ & $3(1)$ \\
\hline C34 & $38(2)$ & $39(2)$ & $51(2)$ & $13(2)$ & $20(2)$ & $18(2)$ \\
\hline C35 & $31(2)$ & $30(2)$ & $15(1)$ & $1(1)$ & $-1(1)$ & $6(1)$ \\
\hline C36 & $53(2)$ & $49(2)$ & $20(2)$ & $-5(2)$ & $-6(2)$ & $25(2)$ \\
\hline C37 & $60(2)$ & $52(2)$ & $20(2)$ & $11(2)$ & $4(2)$ & $-9(2)$ \\
\hline C38 & $50(2)$ & $31(2)$ & $20(2)$ & $-2(1)$ & $-3(1)$ & $10(2)$ \\
\hline C39 & $22(1)$ & $26(2)$ & $24(2)$ & $-1(1)$ & $9(1)$ & $0(1)$ \\
\hline $\mathrm{C} 40$ & $35(2)$ & $48(2)$ & $26(2)$ & $-2(2)$ & $14(1)$ & $8(2)$ \\
\hline $\mathrm{C} 41$ & $34(2)$ & $30(2)$ & $34(2)$ & $-2(1)$ & $16(1)$ & $3(1)$ \\
\hline $\mathrm{C} 42$ & $30(2)$ & $33(2)$ & $46(2)$ & $-5(2)$ & $19(2)$ & $-2(1)$ \\
\hline $\mathrm{C} 43$ & $20(1)$ & $34(2)$ & $18(1)$ & $-1(1)$ & $2(1)$ & $3(1)$ \\
\hline $\mathrm{C} 44$ & $31(2)$ & $40(2)$ & $50(2)$ & $-17(2)$ & $-7(2)$ & $15(1)$ \\
\hline $\mathrm{C} 45$ & $24(2)$ & $46(2)$ & $43(2)$ & $-14(2)$ & $-1(1)$ & $0(1)$ \\
\hline $\mathrm{C} 46$ & $36(2)$ & $69(3)$ & $29(2)$ & $12(2)$ & $-4(2)$ & $2(2)$ \\
\hline C47 & $28(1)$ & $16(1)$ & $30(2)$ & $2(1)$ & $9(1)$ & $-2(1)$ \\
\hline $\mathrm{C} 48$ & $79(3)$ & $19(2)$ & $48(2)$ & $-4(2)$ & $36(2)$ & $-5(2)$ \\
\hline C49 & $39(2)$ & $26(2)$ & $30(2)$ & $4(1)$ & $14(1)$ & $-4(1)$ \\
\hline C50 & $37(2)$ & $29(2)$ & $71(3)$ & $26(2)$ & $10(2)$ & $1(1)$ \\
\hline C51 & $31(2)$ & $22(1)$ & $15(1)$ & $3(1)$ & $3(1)$ & $2(1)$ \\
\hline C52 & $38(2)$ & $31(2)$ & $17(2)$ & 1(1) & $-6(1)$ & $1(1)$ \\
\hline C53 & $42(2)$ & $30(2)$ & $16(2)$ & $3(1)$ & $9(1)$ & $5(1)$ \\
\hline C54 & $39(2)$ & $25(2)$ & $24(2)$ & $4(1)$ & $12(1)$ & $5(1)$ \\
\hline C55 & $29(2)$ & $45(2)$ & $22(2)$ & $11(2)$ & $12(2)$ & $17(2)$ \\
\hline C56 & $50(3)$ & $64(3)$ & $65(5)$ & $44(3)$ & $43(3)$ & $32(3)$ \\
\hline C57 & $49(4)$ & $93(4)$ & $22(2)$ & $-16(2)$ & $3(2)$ & $37(3)$ \\
\hline C58 & $32(2)$ & $40(2)$ & $25(3)$ & $6(2)$ & $11(2)$ & $13(2)$ \\
\hline C55' & $29(2)$ & $45(2)$ & $22(2)$ & $11(2)$ & $12(2)$ & $17(2)$ \\
\hline C56' & $50(3)$ & $64(3)$ & $65(5)$ & $44(3)$ & $43(3)$ & $32(3)$ \\
\hline C57' & $49(4)$ & $93(4)$ & $22(2)$ & $-16(2)$ & $3(2)$ & $37(3)$ \\
\hline C58' & $32(2)$ & $40(2)$ & $25(3)$ & $6(2)$ & $11(2)$ & $13(2)$ \\
\hline
\end{tabular}




\begin{tabular}{lcccccc} 
O1 & $56(2)$ & $57(2)$ & $44(2)$ & $-4(1)$ & $18(1)$ & $-12(1)$ \\
$\mathrm{C} 59$ & $65(3)$ & $50(2)$ & $36(2)$ & $15(2)$ & $22(2)$ & $8(2)$ \\
$\mathrm{C} 60$ & $50(2)$ & $76(3)$ & $42(2)$ & $-13(2)$ & $22(2)$ & $-7(2)$ \\
$\mathrm{O} 2$ & $42(2)$ & $116(3)$ & $44(2)$ & $3(2)$ & $12(1)$ & $6(2)$ \\
$\mathrm{C} 61$ & $39(2)$ & $86(3)$ & $32(2)$ & $14(2)$ & $11(2)$ & $11(2)$ \\
$\mathrm{C} 62$ & $74(3)$ & $65(3)$ & $34(2)$ & $-9(2)$ & $24(2)$ & $9(2)$ \\
O3 & $28(1)$ & $36(1)$ & $33(1)$ & $7(1)$ & $-2(1)$ & $5(1)$ \\
$\mathrm{C} 63$ & $23(2)$ & $47(2)$ & $30(2)$ & $10(2)$ & $0(1)$ & $-3(1)$ \\
$\mathrm{C} 64$ & $34(2)$ & $53(2)$ & $31(2)$ & $3(2)$ & $2(2)$ & $-6(2)$ \\
O4 & $28(1)$ & $50(2)$ & $43(2)$ & $9(1)$ & $-5(1)$ & $6(1)$ \\
C65 & $34(2)$ & $56(2)$ & $43(2)$ & $3(2)$ & $4(2)$ & $10(2)$ \\
C66 & $36(2)$ & $44(2)$ & $42(2)$ & $-6(2)$ & $-1(2)$ & $8(2)$ \\
& & & & & & \\
\hline
\end{tabular}


Table S11. Hydrogen coordinates $\left(\times 10^{4}\right)$ and isotropic displacement parameters $\left(\AA^{2} \times 10^{3}\right)$ for neijk04.

\begin{tabular}{|c|c|c|c|c|}
\hline & $\mathrm{x}$ & $\mathrm{y}$ & $\mathrm{z}$ & $\mathrm{U}(\mathrm{eq})$ \\
\hline $\mathrm{H} 1 \mathrm{~A}$ & 6239 & 2314 & 7140 & 23 \\
\hline H1B & 7143 & 2176 & 7850 & 23 \\
\hline $\mathrm{H} 2 \mathrm{~A}$ & 8044 & 2803 & 7559 & 23 \\
\hline $\mathrm{H} 2 \mathrm{~B}$ & 7003 & 3005 & 7613 & 23 \\
\hline H4 & 6583 & 3955 & 5719 & 23 \\
\hline H6 & 8896 & 4773 & 6609 & 24 \\
\hline H8 & 8995 & 3397 & 7144 & 21 \\
\hline H10 & 6096 & 3091 & 4708 & 23 \\
\hline H12 & 3391 & 3439 & 4873 & 25 \\
\hline H14 & 5427 & 3209 & 6915 & 21 \\
\hline H16 & 8994 & 1514 & 6130 & 24 \\
\hline H18 & 10525 & 855 & 8099 & 25 \\
\hline H20 & 8177 & 1610 & 8278 & 22 \\
\hline $\mathrm{H} 22$ & 7209 & 942 & 7006 & 21 \\
\hline $\mathrm{H} 24$ & 4417 & 646 & 5983 & 25 \\
\hline H26 & 5489 & 1920 & 5691 & 23 \\
\hline $\mathrm{H} 28 \mathrm{~A}$ & 10121 & 4860 & 7652 & 101 \\
\hline $\mathrm{H} 28 \mathrm{~B}$ & 10585 & 4693 & 6914 & 101 \\
\hline $\mathrm{H} 28 \mathrm{C}$ & 11186 & 4639 & 7829 & 101 \\
\hline $\mathrm{H} 29 \mathrm{~A}$ & 9608 & 4264 & 8561 & 65 \\
\hline H29B & 10701 & 4078 & 8742 & 65 \\
\hline $\mathrm{H} 29 \mathrm{C}$ & 9822 & 3726 & 8429 & 65 \\
\hline H30A & 11446 & 3834 & 7596 & 55 \\
\hline H30B & 10812 & 3846 & 6688 & 55 \\
\hline H30C & 10564 & 3478 & 7327 & 55 \\
\hline $\mathrm{H} 32 \mathrm{~A}$ & 8194 & 5236 & 5439 & 51 \\
\hline H32B & 7921 & 5366 & 6285 & 51 \\
\hline $\mathrm{H} 32 \mathrm{C}$ & 7249 & 5541 & 5449 & 51 \\
\hline $\mathrm{H} 33 \mathrm{~A}$ & 7213 & 4665 & 4513 & 48 \\
\hline H33B & 6310 & 5003 & 4503 & 48 \\
\hline
\end{tabular}




\begin{tabular}{|c|c|c|c|c|}
\hline $\mathrm{H} 33 \mathrm{C}$ & 6239 & 4464 & 4721 & 48 \\
\hline $\mathrm{H} 34 \mathrm{~A}$ & 6455 & 5020 & 6645 & 62 \\
\hline H34B & 5807 & 4658 & 6037 & 62 \\
\hline $\mathrm{H} 34 \mathrm{C}$ & 5822 & 5197 & 5795 & 62 \\
\hline H36A & 2937 & 3381 & 3600 & 64 \\
\hline H36B & 3287 & 3551 & 2806 & 64 \\
\hline $\mathrm{H} 36 \mathrm{C}$ & 3477 & 3871 & 3601 & 64 \\
\hline H37A & 5818 & 3424 & 3546 & 68 \\
\hline H37B & 5252 & 3898 & 3629 & 68 \\
\hline $\mathrm{H} 37 \mathrm{C}$ & 5005 & 3608 & 2799 & 68 \\
\hline H38A & 3840 & 2647 & 3526 & 53 \\
\hline H38B & 4981 & 2657 & 3565 & 53 \\
\hline $\mathrm{H} 38 \mathrm{C}$ & 4240 & 2834 & 2767 & 53 \\
\hline $\mathrm{H} 40 \mathrm{~A}$ & 4409 & 3668 & 7520 & 53 \\
\hline H40B & 4216 & 3119 & 7522 & 53 \\
\hline $\mathrm{H} 40 \mathrm{C}$ & 3394 & 3475 & 7655 & 53 \\
\hline $\mathrm{H} 41 \mathrm{~A}$ & 3618 & 4155 & 6351 & 48 \\
\hline H41B & 2613 & 3995 & 6557 & 48 \\
\hline $\mathrm{H} 41 \mathrm{C}$ & 2787 & 3934 & 5656 & 48 \\
\hline $\mathrm{H} 42 \mathrm{~A}$ & 2958 & 2763 & 6379 & 53 \\
\hline H42B & 2405 & 3091 & 5660 & 53 \\
\hline $\mathrm{H} 42 \mathrm{C}$ & 2197 & 3141 & 6552 & 53 \\
\hline $\mathrm{H} 44 \mathrm{~A}$ & 3938 & 2111 & 5820 & 64 \\
\hline H44B & 3306 & 1747 & 6205 & 64 \\
\hline $\mathrm{H} 44 \mathrm{C}$ & 2854 & 1993 & 5356 & 64 \\
\hline $\mathrm{H} 45 \mathrm{~A}$ & 3293 & 823 & 4815 & 58 \\
\hline $\mathrm{H} 45 \mathrm{~B}$ & 2450 & 1199 & 4781 & 58 \\
\hline $\mathrm{H} 45 \mathrm{C}$ & 2957 & 966 & 5628 & 58 \\
\hline H46A & 4296 & 1378 & 4191 & 70 \\
\hline H46B & 4360 & 1909 & 4486 & 70 \\
\hline $\mathrm{H} 46 \mathrm{C}$ & 3334 & 1688 & 4069 & 70 \\
\hline $\mathrm{H} 48 \mathrm{~A}$ & 5143 & -82 & 5833 & 68 \\
\hline H48B & 5854 & -447 & 6382 & 68 \\
\hline $\mathrm{H} 48 \mathrm{C}$ & 6278 & -74 & 5845 & 68 \\
\hline H49A & 4558 & 173 & 7074 & 46 \\
\hline H49B & 5330 & 333 & 7868 & 46 \\
\hline
\end{tabular}




\begin{tabular}{|c|c|c|c|c|}
\hline H49C & 5274 & -201 & 7592 & 46 \\
\hline H50A & 7456 & 197 & 7098 & 69 \\
\hline H50B & 7018 & -188 & 7602 & 69 \\
\hline H50C & 7062 & 345 & 7883 & 69 \\
\hline H52A & 10678 & 1163 & 10241 & 46 \\
\hline H52B & 10558 & 1600 & 9639 & 46 \\
\hline $\mathrm{H} 52 \mathrm{C}$ & 11050 & 1131 & 9411 & 46 \\
\hline H53A & 8150 & 1154 & 9389 & 43 \\
\hline H53B & 8778 & 1620 & 9594 & 43 \\
\hline H53C & 8941 & 1195 & 10222 & 43 \\
\hline $\mathrm{H} 54 \mathrm{~A}$ & 8889 & 430 & 9022 & 43 \\
\hline H54B & 9642 & 457 & 9879 & 43 \\
\hline $\mathrm{H} 54 \mathrm{C}$ & 10028 & 408 & 9058 & 43 \\
\hline H56A & 10470 & 1610 & 5833 & 83 \\
\hline H56B & 11453 & 1330 & 5848 & 83 \\
\hline H56C & 11320 & 1615 & 6632 & 83 \\
\hline H57A & 9473 & 890 & 5457 & 84 \\
\hline H57B & 9701 & 429 & 5997 & 84 \\
\hline H57C & 10430 & 594 & 5447 & 84 \\
\hline H58A & 11745 & 870 & 7422 & 47 \\
\hline H58B & 11818 & 610 & 6599 & 47 \\
\hline $\mathrm{H} 58 \mathrm{C}$ & 11125 & 409 & 7152 & 47 \\
\hline H56D & 10876 & 1768 & 6406 & 83 \\
\hline H56E & 11709 & 1463 & 6151 & 83 \\
\hline $\mathrm{H} 56 \mathrm{~F}$ & 11785 & 1598 & 7083 & 83 \\
\hline H57D & 9992 & 1073 & 5521 & 84 \\
\hline H57E & 9946 & 582 & 5958 & 84 \\
\hline H57F & 10897 & 725 & 5645 & 84 \\
\hline H58D & 11707 & 841 & 7759 & 47 \\
\hline H58E & 12127 & 736 & 6969 & 47 \\
\hline H58F & 11280 & 418 & 7171 & 47 \\
\hline H59A & 6859 & 3806 & 9614 & 59 \\
\hline H59B & 6068 & 4147 & 9854 & 59 \\
\hline $\mathrm{H} 60 \mathrm{~A}$ & 6925 & 4793 & 9562 & 65 \\
\hline H60B & 7606 & 4475 & 10233 & 65 \\
\hline H61A & 7896 & 4419 & 7981 & 62 \\
\hline
\end{tabular}




$\begin{array}{lrlll}\text { H61B } & 7101 & 4760 & 8214 & 62 \\ \text { H62A } & 6354 & 4096 & 7582 & 67 \\ \text { H62B } & 7024 & 3773 & 8250 & 67 \\ \text { H63A } & 9735 & 2315 & 8923 & 41 \\ \text { H63B } & 9468 & 2848 & 9052 & 41 \\ \text { H64A } & 11109 & 3055 & 9319 & 48 \\ \text { H64B } & 10956 & 2647 & 9927 & 48 \\ \text { H65A } & 12043 & 2334 & 8035 & 54 \\ \text { H65B } & 11747 & 2862 & 8168 & 54 \\ \text { H66A } & 10552 & 2502 & 7167 & 51 \\ \text { H66B } & 10404 & 2110 & 7805 & 51 \\ \end{array}$


Table S12. Torsion angles $\left[^{\circ}\right]$ for neijk04.

\begin{tabular}{|c|c|c|c|}
\hline C15-P2-C1-C2 & $95.7(2)$ & C9-C10-C11-C35 & $179.4(2)$ \\
\hline $\mathrm{C} 21-\mathrm{P} 2-\mathrm{C} 1-\mathrm{C} 2$ & $-155.27(19)$ & $\mathrm{C} 10-\mathrm{C} 11-\mathrm{C} 12-\mathrm{C} 13$ & $1.5(4)$ \\
\hline $\mathrm{Fe} 1-\mathrm{P} 2-\mathrm{C} 1-\mathrm{C} 2$ & $-27.0(2)$ & C35-C11-C12-C13 & $179.8(3)$ \\
\hline P2-C1-C2-P1 & $50.1(2)$ & C11-C12-C13-C14 & $0.1(4)$ \\
\hline C9-P1-C2-C1 & $70.6(2)$ & C11-C12-C13-C39 & $179.2(3)$ \\
\hline C3-P1-C2-C1 & $-178.80(18)$ & C12-C13-C14-C9 & $-1.0(4)$ \\
\hline $\mathrm{Fe} 1-\mathrm{P} 1-\mathrm{C} 2-\mathrm{C} 1$ & $-48.05(19)$ & C39-C13-C14-C9 & $180.0(2)$ \\
\hline C9-P1-C3-C8 & $165.7(2)$ & C10-C9-C14-C13 & $0.3(4)$ \\
\hline C2-P1-C3-C8 & $51.9(2)$ & P1-C9-C14-C13 & $175.2(2)$ \\
\hline Fe1-P1-C3-C8 & $-66.8(2)$ & C21-P2-C15-C16 & $95.6(2)$ \\
\hline C9-P1-C3-C4 & $-17.2(3)$ & C1-P2-C15-C16 & $-157.3(2)$ \\
\hline C2-P1-C3-C4 & $-131.0(2)$ & Fe1-P2-C15-C16 & $-38.3(2)$ \\
\hline Fe1-P1-C3-C4 & $110.3(2)$ & $\mathrm{C} 21-\mathrm{P} 2-\mathrm{C} 15-\mathrm{C} 20$ & $-79.0(2)$ \\
\hline $\mathrm{C} 8-\mathrm{C} 3-\mathrm{C} 4-\mathrm{C} 5$ & $0.9(4)$ & C1-P2-C15-C20 & 28.1(3) \\
\hline $\mathrm{P} 1-\mathrm{C} 3-\mathrm{C} 4-\mathrm{C} 5$ & $-176.2(2)$ & Fe1-P2-C15-C20 & 147.11(19) \\
\hline C3-C4-C5-C6 & $-0.1(4)$ & C20-C15-C16-C17 & $0.3(4)$ \\
\hline C3-C4-C5-C31 & $-179.6(2)$ & P2-C15-C16-C17 & $-174.5(2)$ \\
\hline C4-C5-C6-C7 & $-1.2(4)$ & C15-C16-C17-C18 & $0.3(4)$ \\
\hline C31-C5-C6-C7 & $178.3(3)$ & C15-C16-C17-C55 & $-179.3(4)$ \\
\hline C5-C6-C7-C8 & $1.7(4)$ & C15-C16-C17-C55' & $-161(2)$ \\
\hline C5-C6-C7-C27 & $-176.4(3)$ & C16-C17-C18-C19 & $-0.6(4)$ \\
\hline $\mathrm{C} 4-\mathrm{C} 3-\mathrm{C} 8-\mathrm{C} 7$ & $-0.4(4)$ & C55-C17-C18-C19 & $178.9(4)$ \\
\hline $\mathrm{P} 1-\mathrm{C} 3-\mathrm{C} 8-\mathrm{C} 7$ & $176.7(2)$ & C55'-C17-C18-C19 & $163.0(17)$ \\
\hline $\mathrm{C} 6-\mathrm{C} 7-\mathrm{C} 8-\mathrm{C} 3$ & $-0.8(4)$ & C17-C18-C19-C20 & $0.4(4)$ \\
\hline $\mathrm{C} 27-\mathrm{C} 7-\mathrm{C} 8-\mathrm{C} 3$ & $177.3(2)$ & C17-C18-C19-C51 & $179.7(2)$ \\
\hline C3-P1-C9-C10 & $90.2(2)$ & C18-C19-C20-C15 & $0.2(4)$ \\
\hline C2-P1-C9-C10 & $-159.47(19)$ & C51-C19-C20-C15 & $-179.2(2)$ \\
\hline Fe1-P1-C9-C10 & $-44.6(2)$ & C16-C15-C20-C19 & $-0.5(4)$ \\
\hline C3-P1-C9-C14 & $-84.9(2)$ & P2-C15-C20-C19 & $173.9(2)$ \\
\hline C2-P1-C9-C14 & $25.4(3)$ & C15-P2-C21-C26 & $-176.4(2)$ \\
\hline Fe1-P1-C9-C14 & $140.2(2)$ & $\mathrm{C} 1-\mathrm{P} 2-\mathrm{C} 21-\mathrm{C} 26$ & $70.5(2)$ \\
\hline C14-C9-C10-C11 & $1.4(4)$ & Fe1-P2-C21-C26 & $-47.6(2)$ \\
\hline P1-C9-C10-C11 & $-174.0(2)$ & $\mathrm{C} 15-\mathrm{P} 2-\mathrm{C} 21-\mathrm{C} 22$ & $5.9(2)$ \\
\hline C9-C10-C11-C12 & $-2.2(4)$ & C1-P2-C21-C22 & $-107.2(2)$ \\
\hline
\end{tabular}




\begin{tabular}{|c|c|c|c|}
\hline Fe1-P2-C21-C22 & $134.70(19)$ & C12-C13-C39-C42 & $62.8(3)$ \\
\hline $\mathrm{C} 26-\mathrm{C} 21-\mathrm{C} 22-\mathrm{C} 23$ & $-0.5(4)$ & $\mathrm{C} 24-\mathrm{C} 25-\mathrm{C} 43-\mathrm{C} 44$ & $116.8(3)$ \\
\hline $\mathrm{P} 2-\mathrm{C} 21-\mathrm{C} 22-\mathrm{C} 23$ & $177.1(2)$ & C26-C25-C43-C44 & $-63.2(3)$ \\
\hline $\mathrm{C} 21-\mathrm{C} 22-\mathrm{C} 23-\mathrm{C} 24$ & $-0.3(4)$ & $\mathrm{C} 24-\mathrm{C} 25-\mathrm{C} 43-\mathrm{C} 46$ & $-121.2(3)$ \\
\hline $\mathrm{C} 21-\mathrm{C} 22-\mathrm{C} 23-\mathrm{C} 47$ & $-180.0(2)$ & C26-C25-C43-C46 & $58.8(3)$ \\
\hline $\mathrm{C} 22-\mathrm{C} 23-\mathrm{C} 24-\mathrm{C} 25$ & $0.5(4)$ & $\mathrm{C} 24-\mathrm{C} 25-\mathrm{C} 43-\mathrm{C} 45$ & $-3.3(4)$ \\
\hline C47-C23-C24-C25 & $-179.8(2)$ & C26-C25-C43-C45 & $176.7(2)$ \\
\hline $\mathrm{C} 23-\mathrm{C} 24-\mathrm{C} 25-\mathrm{C} 26$ & $0.1(4)$ & $\mathrm{C} 22-\mathrm{C} 23-\mathrm{C} 47-\mathrm{C} 50$ & $3.3(4)$ \\
\hline $\mathrm{C} 23-\mathrm{C} 24-\mathrm{C} 25-\mathrm{C} 43$ & $-180.0(2)$ & C24-C23-C47-C50 & $-176.4(3)$ \\
\hline $\mathrm{C} 22-\mathrm{C} 21-\mathrm{C} 26-\mathrm{C} 25$ & $1.2(4)$ & $\mathrm{C} 22-\mathrm{C} 23-\mathrm{C} 47-\mathrm{C} 48$ & $-117.4(3)$ \\
\hline P2-C21-C26-C25 & $-176.6(2)$ & $\mathrm{C} 24-\mathrm{C} 23-\mathrm{C} 47-\mathrm{C} 48$ & $62.9(3)$ \\
\hline $\mathrm{C} 24-\mathrm{C} 25-\mathrm{C} 26-\mathrm{C} 21$ & $-1.0(4)$ & C22-C23-C47-C49 & $122.9(3)$ \\
\hline $\mathrm{C} 43-\mathrm{C} 25-\mathrm{C} 26-\mathrm{C} 21$ & $179.1(2)$ & $\mathrm{C} 24-\mathrm{C} 23-\mathrm{C} 47-\mathrm{C} 49$ & $-56.8(3)$ \\
\hline C6-C7-C27-C28 & $-8.2(4)$ & C20-C19-C51-C53 & $1.0(4)$ \\
\hline C8-C7-C27-C28 & $173.6(3)$ & C18-C19-C51-C53 & $-178.4(2)$ \\
\hline C6-C7-C27-C29 & $113.2(3)$ & C20-C19-C51-C52 & $-119.5(3)$ \\
\hline C8-C7-C27-C29 & $-65.0(3)$ & C18-C19-C51-C52 & $61.2(3)$ \\
\hline C6-C7-C27-C30 & $-128.1(3)$ & C20-C19-C51-C54 & $120.6(3)$ \\
\hline C8-C7-C27-C30 & $53.8(3)$ & C18-C19-C51-C54 & $-58.8(3)$ \\
\hline C4-C5-C31-C32 & $-172.9(3)$ & C16-C17-C55-C57 & $-60.4(4)$ \\
\hline C6-C5-C31-C32 & $7.6(4)$ & C18-C17-C55-C57 & $120.1(4)$ \\
\hline C4-C5-C31-C34 & $66.7(3)$ & C55'-C17-C55-C57 & $176(5)$ \\
\hline C6-C5-C31-C34 & $-112.7(3)$ & C16-C17-C55-C58 & $178.9(3)$ \\
\hline C4-C5-C31-C33 & $-52.9(3)$ & C18-C17-C55-C58 & $-0.6(5)$ \\
\hline C6-C5-C31-C33 & $127.7(3)$ & C55'-C17-C55-C58 & $55(4)$ \\
\hline C12-C11-C35-C38 & $-106.0(3)$ & C16-C17-C55-C56 & $60.4(4)$ \\
\hline C10-C11-C35-C38 & $72.3(3)$ & C18-C17-C55-C56 & $-119.1(4)$ \\
\hline C12-C11-C35-C37 & $134.5(3)$ & C55'-C17-C55-C56 & $-64(4)$ \\
\hline C10-C11-C35-C37 & $-47.2(4)$ & C16-C17-C55'-C57' & $-61(2)$ \\
\hline C12-C11-C35-C36 & $14.3(4)$ & C18-C17-C55'-C57' & $138(2)$ \\
\hline C10-C11-C35-C36 & $-167.4(3)$ & C55-C17-C55'-C57' & $5(4)$ \\
\hline C14-C13-C39-C40 & $2.7(4)$ & C16-C17-C55'-C58' & $-179.6(19)$ \\
\hline C12-C13-C39-C40 & $-176.3(3)$ & C18-C17-C55'-C58' & $19(3)$ \\
\hline C14-C13-C39-C41 & $122.4(3)$ & C55-C17-C55'-C58' & $-114(5)$ \\
\hline C12-C13-C39-C41 & $-56.5(3)$ & C16-C17-C55'-C56' & $58(3)$ \\
\hline C14-C13-C39-C42 & $-118.2(3)$ & C18-C17-C55'-C56' & $-104(2)$ \\
\hline
\end{tabular}


C55-C17-C55'-C56'

C62-O1-C59-C60

O1-C59-C60-O2

C59-C60-O2-C61

C60-O2-C61-C62

C59-O1-C62-C61

O2-C61-C62-O1
124(6)

58.2(4)

$-59.0(5)$

$57.4(5)$

$-57.4(5)$

$-57.7(4)$

58.6(4)
C66-O3-C63-C64

57.3(4)

O3-C63-C64-O4

$-57.1(4)$

C63-C64-O4-C65

56.5(4)

C64-O4-C65-C66

$-58.0(4)$

C63-O3-C66-C65

$-58.7(4)$

O4-C65-C66-O3

60.3(4) 


\section{3 $\mathrm{FeCl}_{2}(\mathrm{dpbz})$}

REFERENCE NUMBER: neijk31

CRYSTAL STRUCTURE REPORT

$$
\mathrm{C}_{30} \mathrm{H}_{24} \mathrm{Cl}_{2} \mathrm{Fe} \mathrm{P}_{2}
$$

or

$(\mathrm{dpbz}) \mathrm{FeCl}_{2} \cdot \mathrm{xSolvent}$

Report prepared for:

J. Bailey, J. Kneebone, Prof. M. Neidig

September 28, 2014

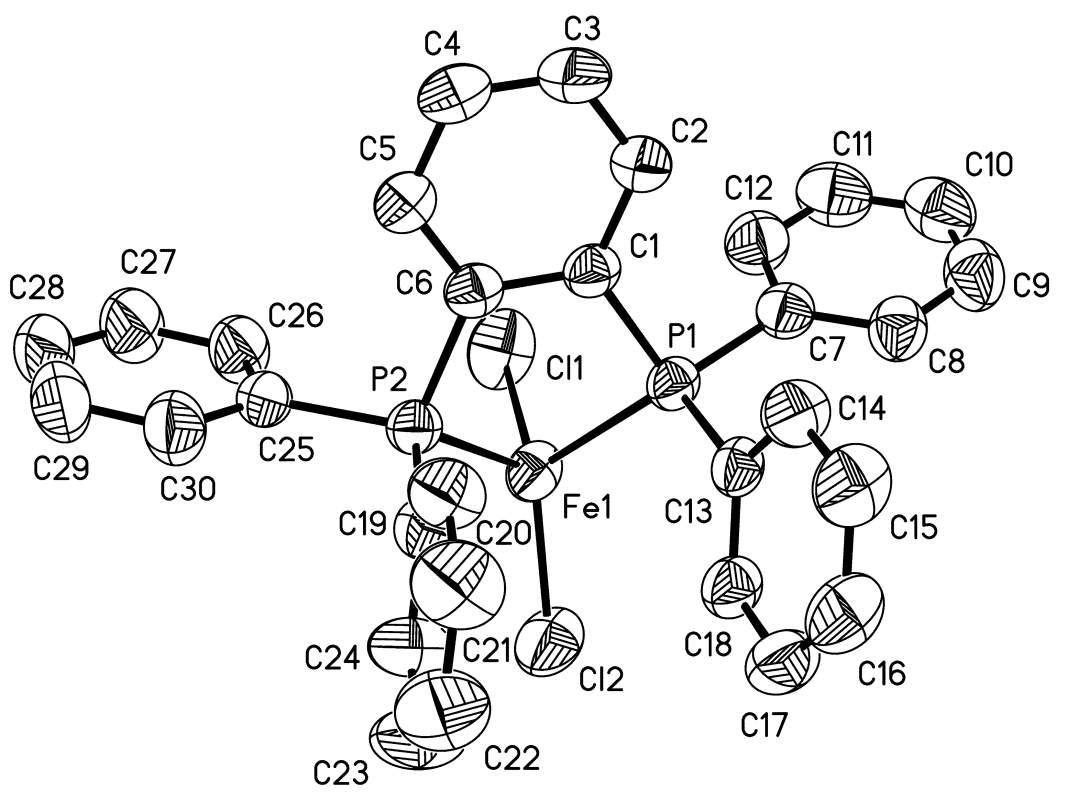

William W. Brennessel

X-ray Crystallographic Facility

Department of Chemistry, University of Rochester

120 Trustee Road

Rochester, NY 14627 


\section{Data collection}

A crystal $\left(0.24 \times 0.20 \times 0.12 \mathrm{~mm}^{3}\right)$ was placed onto the tip of a thin glass optical fiber and mounted on a Bruker SMART APEX II CCD platform diffractometer for a data collection at $173(2) \mathrm{K} .{ }^{1}$ A preliminary set of cell constants and an orientation matrix were calculated from reflections harvested from three orthogonal wedges of reciprocal space. The full data collection was carried out using MoKa radiation (graphite monochromator) with a frame time of 45 seconds and a detector distance of $4.02 \mathrm{~cm}$. A randomly oriented region of reciprocal space was surveyed: six major sections of frames were collected with $0.50^{\circ}$ steps in $\omega$ at six different $\phi$ settings and a detector position of $-38^{\circ}$ in $2 \theta$. The intensity data were corrected for absorption. ${ }^{2}$ Final cell constants were calculated from the xyz centroids of 4091 strong reflections from the actual data collection after integration. ${ }^{3}$ See Table 1 for additional crystal and refinement information.

Structure solution and refinement

The structure was solved using SIR2011 4 and refined using SHELXL-2014/7.5 The space group $P$-1 was determined based on intensity statistics. A direct-methods solution was calculated which provided most nonhydrogen atoms from the E-map. Full-matrix least squares / difference Fourier cycles were performed which located the remaining non-hydrogen atoms. All non-hydrogen atoms were refined with anisotropic displacement parameters. All hydrogen atoms were placed in ideal positions and refined as riding atoms with relative isotropic displacement parameters.

Highly disordered solvent (perhaps toluene) was found in a pocket located at a crystallographic inversion center. Reflection contributions from the disordered solvent were fixed and added to the calculated structure factors using the SQUEEZE routine of program Platon, ${ }^{6}$ which determined there to be 59 electrons in $205 \AA^{3}$ that were accounted for per unit cell. Because the exact identity and amount of disordered solvent is not known, no disordered solvent was included in the atom list and molecular formula. Thus all calculations that derive from the molecular formula (e.g., F(000), density, molecular weight, etc.) are known to be incorrect.

The final full matrix least squares refinement converged to $R 1=0.0405\left(F^{2}, I>2 \sigma(I)\right)$ and $w R 2=0.1096\left(F^{2}\right.$, all data).

\section{Structure description}

The structure is the one suggested. The asymmetric unit contains one iron complex in a general position and onehalf of a solvent molecule (see above) on a crystallographic inversion center.

Unless noted otherwise all structural diagrams containing thermal displacement ellipsoids are drawn at the $50 \%$ probability level. 
Data collection, structure solution, and structure refinement were conducted at the X-ray Crystallographic Facility, B51 Hutchison Hall, Department of Chemistry, University of Rochester. All publications arising from this report MUST either 1) include William W. Brennessel as a coauthor or 2) acknowledge William W. Brennessel and the Xray Crystallographic Facility of the Department of Chemistry at the University of Rochester.

1 APEX3, version 2015.5-2; Bruker AXS: Madison, WI, 2015.

2 Sheldrick, G. M. SADABS, version 2014/5; J. Appl. Cryst. 2015, 48, 3-10.

3 SAINT, version 8.34A; Bruker AXS: Madison, WI, 2013.

4 Burla, M. C.; Caliandro, R.; Camalli, M.; Carrozzini, B.; Cascarano, G. L.; Giacovazzo, C.; Mallamo, M.;

Mazzone, A.; Polidori, G.; Spagna, R. SIR2011, version 1.0; J. Appl. Cryst. 2012, 45, 357-361.

5 Sheldrick, G. M. SHELXL-2014/7; Acta. Cryst. 2015, C71, 3-8.

6 Spek, A. L. PLATON, version 07052015; Acta. Cryst. 2015, C71, 9-18.

Some equations of interest:

$$
\begin{gathered}
R_{\text {int }}=\Sigma\left|F_{\mathrm{o}}^{2}-<F_{\mathrm{o}}^{2}>\right| / \Sigma\left|F_{\mathrm{o}}{ }^{2}\right| \\
R 1=\Sigma|| F_{\mathrm{o}}|-| F_{\mathrm{c}}|| / \Sigma\left|F_{\mathrm{o}}\right| \\
w R 2=\left[\Sigma\left[w\left(F_{\mathrm{o}}{ }^{2}-F_{\mathrm{c}}{ }^{2}\right)^{2}\right] / \Sigma\left[w\left(F_{\mathrm{o}}^{2}\right)^{2}\right]\right]^{1 / 2} \\
\text { where } w=1 /\left[\sigma^{2}\left(F_{\mathrm{o}}{ }^{2}\right)+(a P)^{2}+b P\right] \text { and } \\
P=1 / 3 \max \left(0, F_{\mathrm{o}}^{2}\right)+2 / 3 F_{\mathrm{c}}^{2} \\
\text { GOF }=S=\left[\Sigma\left[w\left(F_{\mathrm{o}}{ }^{2}-F_{\mathrm{c}}{ }^{2}\right)^{2}\right] /(m-n)\right]^{1 / 2}
\end{gathered}
$$

where $m=$ number of reflections and $n=$ number of parameters

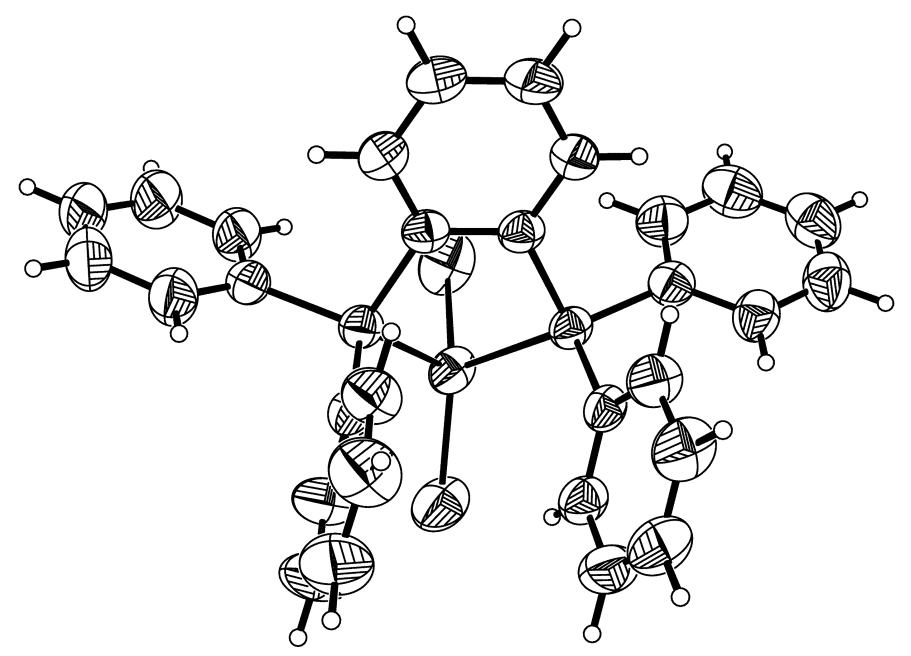


Table S13. Crystal data and structure refinement for neijk31.

\begin{tabular}{|c|c|c|}
\hline Identification code & \multicolumn{2}{|l|}{ neijk31 } \\
\hline Empirical formula & \multicolumn{2}{|c|}{$\mathrm{C} 30 \mathrm{H} 24 \mathrm{Cl} 2 \mathrm{Fe} \mathrm{P} 2$} \\
\hline Formula weight & \multicolumn{2}{|l|}{573.18} \\
\hline Temperature & \multicolumn{2}{|l|}{$173(2) \mathrm{K}$} \\
\hline Wavelength & \multicolumn{2}{|l|}{$0.71073 \AA$} \\
\hline Crystal system & \multicolumn{2}{|l|}{ triclinic } \\
\hline Space group & \multicolumn{2}{|l|}{$P-1$} \\
\hline \multirow[t]{3}{*}{ Unit cell dimensions } & $a=9.381(2) \AA$ & $\alpha=82.096(5)^{\circ}$ \\
\hline & $b=10.138(2) \AA$ & $\beta=81.479(5)^{\circ}$ \\
\hline & $c=17.591(4) \AA$ & $\gamma=68.716(5)^{\circ}$ \\
\hline Volume & \multicolumn{2}{|l|}{$1535.4(6) \AA^{3}$} \\
\hline$Z$ & \multicolumn{2}{|l|}{2} \\
\hline Density (calculated) & \multicolumn{2}{|l|}{$1.240 \mathrm{Mg} / \mathrm{m}^{3}$} \\
\hline Absorption coefficient & \multicolumn{2}{|l|}{$0.785 \mathrm{~mm}^{-1}$} \\
\hline$F(000)$ & \multicolumn{2}{|l|}{588} \\
\hline Crystal color, morphology & \multicolumn{2}{|l|}{ colorless, block } \\
\hline Crystal size & \multicolumn{2}{|c|}{$0.24 \times 0.20 \times 0.12 \mathrm{~mm}^{3}$} \\
\hline Theta range for data collection & \multicolumn{2}{|l|}{2.343 to $28.348^{\circ}$} \\
\hline Index ranges & \multicolumn{2}{|c|}{$-12 \leq h \leq 12,-13 \leq k \leq 13,-23 \leq l \leq 23$} \\
\hline Reflections collected & \multicolumn{2}{|l|}{32606} \\
\hline Independent reflections & \multicolumn{2}{|c|}{$7663[R($ int $)=0.0433]$} \\
\hline Observed reflections & \multicolumn{2}{|l|}{5295} \\
\hline Completeness to theta $=28.283^{\circ}$ & \multicolumn{2}{|l|}{$99.9 \%$} \\
\hline Absorption correction & \multicolumn{2}{|l|}{ Multi-scan } \\
\hline Max. and min. transmission & \multicolumn{2}{|c|}{0.7457 and 0.6560} \\
\hline Refinement method & \multicolumn{2}{|c|}{ Full-matrix least-squares on $F^{2}$} \\
\hline Data / restraints / parameters & \multicolumn{2}{|l|}{$7663 / 0 / 316$} \\
\hline Goodness-of-fit on $F^{2}$ & \multicolumn{2}{|l|}{1.012} \\
\hline Final $R$ indices $[I>2 \operatorname{sigma}(I)]$ & \multicolumn{2}{|c|}{$R 1=0.0405, w R 2=0.0969$} \\
\hline$R$ indices (all data) & \multicolumn{2}{|c|}{$R 1=0.0657, w R 2=0.1096$} \\
\hline Largest diff. peak and hole & \multicolumn{2}{|c|}{0.469 and -0.289 e..$\AA^{-3}$} \\
\hline
\end{tabular}


Table S14. Atomic coordinates $\left(\times 10^{4}\right)$ and equivalent isotropic displacement parameters $\left(\AA^{2} \times 10^{3}\right)$ for neijk31. $U_{e q}$ is defined as one third of the trace of the orthogonalized $U_{i j}$ tensor.

\begin{tabular}{|c|c|c|c|c|}
\hline & $\mathrm{x}$ & $\mathrm{y}$ & $\mathrm{z}$ & $\mathrm{U}_{\mathrm{eq}}$ \\
\hline Fe1 & $-969(1)$ & $7580(1)$ & $2110(1)$ & $53(1)$ \\
\hline Cl1 & $-2145(1)$ & $9477(1)$ & 1342(1) & $82(1)$ \\
\hline $\mathrm{Cl} 2$ & $-2126(1)$ & $6257(1)$ & $2870(1)$ & $90(1)$ \\
\hline P1 & $1417(1)$ & $6412(1)$ & $1320(1)$ & $43(1)$ \\
\hline $\mathrm{P} 2$ & $808(1)$ & $8251(1)$ & 2714(1) & $45(1)$ \\
\hline $\mathrm{C} 1$ & 2439(2) & $7655(2)$ & 1271(1) & $44(1)$ \\
\hline $\mathrm{C} 2$ & $3465(3)$ & $7816(2)$ & $643(1)$ & $58(1)$ \\
\hline C3 & 4247(3) & 8731(3) & 641(2) & $70(1)$ \\
\hline $\mathrm{C} 4$ & 3999(3) & $9524(3)$ & $1255(2)$ & $69(1)$ \\
\hline C5 & 2965(3) & 9395(2) & 1879(1) & $56(1)$ \\
\hline C6 & 2179(2) & $8464(2)$ & 1900(1) & $44(1)$ \\
\hline C7 & 1395(2) & 6181(2) & $317(1)$ & $50(1)$ \\
\hline $\mathrm{C} 8$ & 2099(3) & 4881(3) & $9(1)$ & $58(1)$ \\
\hline C9 & 1977(3) & 4773(3) & $-754(2)$ & $72(1)$ \\
\hline $\mathrm{C} 10$ & 1164(3) & 5919(4) & $-1201(2)$ & $76(1)$ \\
\hline C11 & $445(3)$ & 7204(3) & $-901(2)$ & $80(1)$ \\
\hline $\mathrm{C} 12$ & $557(3)$ & 7344(3) & $-144(2)$ & $68(1)$ \\
\hline C13 & 2732(2) & $4755(2)$ & 1733(1) & $44(1)$ \\
\hline $\mathrm{C} 14$ & 4294(2) & $4268(2)$ & $1480(1)$ & $58(1)$ \\
\hline C15 & $5255(3)$ & 3008(3) & 1804(2) & 71(1) \\
\hline $\mathrm{C} 16$ & 4695(3) & 2239(3) & $2387(2)$ & $73(1)$ \\
\hline C17 & 3158(3) & 2712(3) & 2653(2) & $68(1)$ \\
\hline $\mathrm{C} 18$ & 2176(3) & $3967(2)$ & 2321(1) & $54(1)$ \\
\hline C19 & 1939(3) & $6869(2)$ & $3380(1)$ & $50(1)$ \\
\hline $\mathrm{C} 20$ & $3524(3)$ & $6395(3)$ & $3313(1)$ & $64(1)$ \\
\hline $\mathrm{C} 21$ & $4316(4)$ & $5312(3)$ & $3822(2)$ & $86(1)$ \\
\hline $\mathrm{C} 22$ & $3554(4)$ & 4706(3) & 4395(2) & $93(1)$ \\
\hline $\mathrm{C} 23$ & 1985(4) & $5160(3)$ & 4471(2) & $92(1)$ \\
\hline $\mathrm{C} 24$ & 1168(3) & $6244(3)$ & $3960(2)$ & $71(1)$ \\
\hline $\mathrm{C} 25$ & 202(3) & 9891(2) & $3180(1)$ & $51(1)$ \\
\hline $\mathrm{C} 26$ & $-929(3)$ & $11056(3)$ & 2871(1) & $68(1)$ \\
\hline
\end{tabular}




\begin{tabular}{lrlll}
$\mathrm{C} 27$ & $-1419(4)$ & $12339(3)$ & $3191(2)$ & $85(1)$ \\
$\mathrm{C} 28$ & $-798(4)$ & $12448(3)$ & $3824(2)$ & $89(1)$ \\
$\mathrm{C} 29$ & $309(4)$ & $11304(3)$ & $4141(2)$ & $87(1)$ \\
$\mathrm{C} 30$ & $820(3)$ & $10009(3)$ & $3823(1)$ & $70(1)$ \\
\hline
\end{tabular}


Table S15. Bond lengths $[\AA]$ and angles $\left[{ }^{\circ}\right]$ for neijk31.

\begin{tabular}{|c|c|c|c|}
\hline $\mathrm{Fe}(1)-\mathrm{Cl}(2)$ & $2.2132(8)$ & $\mathrm{C}(14)-\mathrm{H}(14)$ & 0.9500 \\
\hline $\mathrm{Fe}(1)-\mathrm{Cl}(1)$ & $2.2190(8)$ & $C(15)-C(16)$ & $1.362(4)$ \\
\hline $\mathrm{Fe}(1)-\mathrm{P}(2)$ & $2.4330(7)$ & $\mathrm{C}(15)-\mathrm{H}(15)$ & 0.9500 \\
\hline $\mathrm{Fe}(1)-\mathrm{P}(1)$ & $2.4389(7)$ & $C(16)-C(17)$ & $1.374(4)$ \\
\hline $\mathrm{P}(1)-\mathrm{C}(13)$ & $1.816(2)$ & $\mathrm{C}(16)-\mathrm{H}(16)$ & 0.9500 \\
\hline $\mathrm{P}(1)-\mathrm{C}(7)$ & $1.816(2)$ & $\mathrm{C}(17)-\mathrm{C}(18)$ & $1.382(3)$ \\
\hline $\mathrm{P}(1)-\mathrm{C}(1)$ & $1.828(2)$ & $\mathrm{C}(17)-\mathrm{H}(17)$ & 0.9500 \\
\hline $\mathrm{P}(2)-\mathrm{C}(19)$ & $1.816(2)$ & $\mathrm{C}(18)-\mathrm{H}(18)$ & 0.9500 \\
\hline $\mathrm{P}(2)-\mathrm{C}(25)$ & $1.819(2)$ & $C(19)-C(20)$ & $1.379(3)$ \\
\hline $\mathrm{P}(2)-\mathrm{C}(6)$ & $1.822(2)$ & $C(19)-C(24)$ & $1.380(3)$ \\
\hline $\mathrm{C}(1)-\mathrm{C}(2)$ & $1.387(3)$ & $\mathrm{C}(20)-\mathrm{C}(21)$ & $1.377(3)$ \\
\hline$C(1)-C(6)$ & $1.407(3)$ & $\mathrm{C}(20)-\mathrm{H}(20)$ & 0.9500 \\
\hline$C(2)-C(3)$ & $1.376(3)$ & $C(21)-C(22)$ & $1.355(4)$ \\
\hline $\mathrm{C}(2)-\mathrm{H}(2)$ & 0.9500 & $\mathrm{C}(21)-\mathrm{H}(21)$ & 0.9500 \\
\hline$C(3)-C(4)$ & $1.376(4)$ & $\mathrm{C}(22)-\mathrm{C}(23)$ & $1.366(5)$ \\
\hline $\mathrm{C}(3)-\mathrm{H}(3)$ & 0.9500 & $\mathrm{C}(22)-\mathrm{H}(22)$ & 0.9500 \\
\hline$C(4)-C(5)$ & $1.378(3)$ & $C(23)-C(24)$ & $1.388(4)$ \\
\hline $\mathrm{C}(4)-\mathrm{H}(4)$ & 0.9500 & $\mathrm{C}(23)-\mathrm{H}(23)$ & 0.9500 \\
\hline$C(5)-C(6)$ & $1.387(3)$ & $\mathrm{C}(24)-\mathrm{H}(24)$ & 0.9500 \\
\hline $\mathrm{C}(5)-\mathrm{H}(5)$ & 0.9500 & $C(25)-C(26)$ & $1.379(3)$ \\
\hline$C(7)-C(12)$ & $1.386(3)$ & $C(25)-C(30)$ & $1.382(3)$ \\
\hline$C(7)-C(8)$ & $1.388(3)$ & $C(26)-C(27)$ & $1.383(4)$ \\
\hline $\mathrm{C}(8)-\mathrm{C}(9)$ & $1.385(3)$ & $\mathrm{C}(26)-\mathrm{H}(26)$ & 0.9500 \\
\hline $\mathrm{C}(8)-\mathrm{H}(8)$ & 0.9500 & $\mathrm{C}(27)-\mathrm{C}(28)$ & $1.366(4)$ \\
\hline $\mathrm{C}(9)-\mathrm{C}(10)$ & $1.354(4)$ & $\mathrm{C}(27)-\mathrm{H}(27)$ & 0.9500 \\
\hline $\mathrm{C}(9)-\mathrm{H}(9)$ & 0.9500 & $C(28)-C(29)$ & $1.361(4)$ \\
\hline$C(10)-C(11)$ & $1.370(4)$ & $\mathrm{C}(28)-\mathrm{H}(28)$ & 0.9500 \\
\hline $\mathrm{C}(10)-\mathrm{H}(10)$ & 0.9500 & $C(29)-C(30)$ & $1.391(4)$ \\
\hline $\mathrm{C}(11)-\mathrm{C}(12)$ & $1.381(4)$ & $\mathrm{C}(29)-\mathrm{H}(29)$ & 0.9500 \\
\hline $\mathrm{C}(11)-\mathrm{H}(11)$ & 0.9500 & $\mathrm{C}(30)-\mathrm{H}(30)$ & 0.9500 \\
\hline $\mathrm{C}(12)-\mathrm{H}(12)$ & 0.9500 & $\mathrm{Cl}(2)-\mathrm{Fe}(1)-\mathrm{Cl}(1)$ & $124.85(4)$ \\
\hline$C(13)-C(18)$ & $1.377(3)$ & $\mathrm{Cl}(2)-\mathrm{Fe}(1)-\mathrm{P}(2)$ & $115.16(3)$ \\
\hline$C(13)-C(14)$ & $1.390(3)$ & $\mathrm{Cl}(1)-\mathrm{Fe}(1)-\mathrm{P}(2)$ & $105.32(3)$ \\
\hline$C(14)-C(15)$ & $1.373(3)$ & $\mathrm{Cl}(2)-\mathrm{Fe}(1)-\mathrm{P}(1)$ & $119.01(3)$ \\
\hline
\end{tabular}




\begin{tabular}{|c|c|c|c|}
\hline $\mathrm{Cl}(1)-\mathrm{Fe}(1)-\mathrm{P}(1)$ & $102.85(3)$ & $\mathrm{C}(9)-\mathrm{C}(8)-\mathrm{H}(8)$ & 120.1 \\
\hline $\mathrm{P}(2)-\mathrm{Fe}(1)-\mathrm{P}(1)$ & $80.38(3)$ & $\mathrm{C}(7)-\mathrm{C}(8)-\mathrm{H}(8)$ & 120.1 \\
\hline$C(13)-P(1)-C(7)$ & $106.69(10)$ & $C(10)-C(9)-C(8)$ & $120.8(3)$ \\
\hline$C(13)-P(1)-C(1)$ & $103.67(9)$ & $\mathrm{C}(10)-\mathrm{C}(9)-\mathrm{H}(9)$ & 119.6 \\
\hline $\mathrm{C}(7)-\mathrm{P}(1)-\mathrm{C}(1)$ & $104.07(10)$ & $\mathrm{C}(8)-\mathrm{C}(9)-\mathrm{H}(9)$ & 119.6 \\
\hline $\mathrm{C}(13)-\mathrm{P}(1)-\mathrm{Fe}(1)$ & $117.38(7)$ & $\mathrm{C}(9)-\mathrm{C}(10)-\mathrm{C}(11)$ & $120.1(3)$ \\
\hline $\mathrm{C}(7)-\mathrm{P}(1)-\mathrm{Fe}(1)$ & $120.57(7)$ & $\mathrm{C}(9)-\mathrm{C}(10)-\mathrm{H}(10)$ & 120.0 \\
\hline $\mathrm{C}(1)-\mathrm{P}(1)-\mathrm{Fe}(1)$ & $102.08(7)$ & $\mathrm{C}(11)-\mathrm{C}(10)-\mathrm{H}(10)$ & 120.0 \\
\hline $\mathrm{C}(19)-\mathrm{P}(2)-\mathrm{C}(25)$ & $105.84(10)$ & $\mathrm{C}(10)-\mathrm{C}(11)-\mathrm{C}(12)$ & $120.3(3)$ \\
\hline$C(19)-P(2)-C(6)$ & $105.37(10)$ & $\mathrm{C}(10)-\mathrm{C}(11)-\mathrm{H}(11)$ & 119.8 \\
\hline$C(25)-P(2)-C(6)$ & $104.69(10)$ & $\mathrm{C}(12)-\mathrm{C}(11)-\mathrm{H}(11)$ & 119.8 \\
\hline $\mathrm{C}(19)-\mathrm{P}(2)-\mathrm{Fe}(1)$ & $114.56(8)$ & $\mathrm{C}(11)-\mathrm{C}(12)-\mathrm{C}(7)$ & $120.1(2)$ \\
\hline $\mathrm{C}(25)-\mathrm{P}(2)-\mathrm{Fe}(1)$ & $122.11(8)$ & $\mathrm{C}(11)-\mathrm{C}(12)-\mathrm{H}(12)$ & 120.0 \\
\hline $\mathrm{C}(6)-\mathrm{P}(2)-\mathrm{Fe}(1)$ & $102.56(7)$ & $\mathrm{C}(7)-\mathrm{C}(12)-\mathrm{H}(12)$ & 120.0 \\
\hline$C(2)-C(1)-C(6)$ & $119.3(2)$ & $\mathrm{C}(18)-\mathrm{C}(13)-\mathrm{C}(14)$ & $118.9(2)$ \\
\hline $\mathrm{C}(2)-\mathrm{C}(1)-\mathrm{P}(1)$ & $122.38(17)$ & $\mathrm{C}(18)-\mathrm{C}(13)-\mathrm{P}(1)$ & $119.33(15)$ \\
\hline $\mathrm{C}(6)-\mathrm{C}(1)-\mathrm{P}(1)$ & $118.32(15)$ & $\mathrm{C}(14)-\mathrm{C}(13)-\mathrm{P}(1)$ & $121.78(17)$ \\
\hline$C(3)-C(2)-C(1)$ & $120.4(2)$ & $\mathrm{C}(15)-\mathrm{C}(14)-\mathrm{C}(13)$ & $120.2(2)$ \\
\hline $\mathrm{C}(3)-\mathrm{C}(2)-\mathrm{H}(2)$ & 119.8 & $\mathrm{C}(15)-\mathrm{C}(14)-\mathrm{H}(14)$ & 119.9 \\
\hline $\mathrm{C}(1)-\mathrm{C}(2)-\mathrm{H}(2)$ & 119.8 & $\mathrm{C}(13)-\mathrm{C}(14)-\mathrm{H}(14)$ & 119.9 \\
\hline$C(4)-C(3)-C(2)$ & $120.6(2)$ & $C(16)-C(15)-C(14)$ & $120.4(2)$ \\
\hline $\mathrm{C}(4)-\mathrm{C}(3)-\mathrm{H}(3)$ & 119.7 & $\mathrm{C}(16)-\mathrm{C}(15)-\mathrm{H}(15)$ & 119.8 \\
\hline $\mathrm{C}(2)-\mathrm{C}(3)-\mathrm{H}(3)$ & 119.7 & $\mathrm{C}(14)-\mathrm{C}(15)-\mathrm{H}(15)$ & 119.8 \\
\hline$C(3)-C(4)-C(5)$ & $119.8(2)$ & $\mathrm{C}(15)-\mathrm{C}(16)-\mathrm{C}(17)$ & $120.2(2)$ \\
\hline $\mathrm{C}(3)-\mathrm{C}(4)-\mathrm{H}(4)$ & 120.1 & $\mathrm{C}(15)-\mathrm{C}(16)-\mathrm{H}(16)$ & 119.9 \\
\hline $\mathrm{C}(5)-\mathrm{C}(4)-\mathrm{H}(4)$ & 120.1 & $\mathrm{C}(17)-\mathrm{C}(16)-\mathrm{H}(16)$ & 119.9 \\
\hline$C(4)-C(5)-C(6)$ & $120.8(2)$ & $\mathrm{C}(16)-\mathrm{C}(17)-\mathrm{C}(18)$ & $119.8(2)$ \\
\hline $\mathrm{C}(4)-\mathrm{C}(5)-\mathrm{H}(5)$ & 119.6 & $\mathrm{C}(16)-\mathrm{C}(17)-\mathrm{H}(17)$ & 120.1 \\
\hline $\mathrm{C}(6)-\mathrm{C}(5)-\mathrm{H}(5)$ & 119.6 & $\mathrm{C}(18)-\mathrm{C}(17)-\mathrm{H}(17)$ & 120.1 \\
\hline$C(5)-C(6)-C(1)$ & $119.11(19)$ & $\mathrm{C}(13)-\mathrm{C}(18)-\mathrm{C}(17)$ & $120.4(2)$ \\
\hline$C(5)-C(6)-P(2)$ & $122.39(17)$ & $\mathrm{C}(13)-\mathrm{C}(18)-\mathrm{H}(18)$ & 119.8 \\
\hline$C(1)-C(6)-P(2)$ & $118.49(15)$ & $\mathrm{C}(17)-\mathrm{C}(18)-\mathrm{H}(18)$ & 119.8 \\
\hline$C(12)-C(7)-C(8)$ & $119.0(2)$ & $\mathrm{C}(20)-\mathrm{C}(19)-\mathrm{C}(24)$ & $119.2(2)$ \\
\hline $\mathrm{C}(12)-\mathrm{C}(7)-\mathrm{P}(1)$ & 117.91(17) & $\mathrm{C}(20)-\mathrm{C}(19)-\mathrm{P}(2)$ & $122.83(18)$ \\
\hline $\mathrm{C}(8)-\mathrm{C}(7)-\mathrm{P}(1)$ & $122.99(18)$ & $\mathrm{C}(24)-\mathrm{C}(19)-\mathrm{P}(2)$ & $117.99(18)$ \\
\hline $\mathrm{C}(9)-\mathrm{C}(8)-\mathrm{C}(7)$ & $119.8(2)$ & $\mathrm{C}(21)-\mathrm{C}(20)-\mathrm{C}(19)$ & $120.0(3)$ \\
\hline
\end{tabular}




$\begin{array}{llll}\mathrm{C}(21)-\mathrm{C}(20)-\mathrm{H}(20) & 120.0 & \mathrm{C}(30)-\mathrm{C}(25)-\mathrm{P}(2) & 122.97(17) \\ \mathrm{C}(19)-\mathrm{C}(20)-\mathrm{H}(20) & 120.0 & \mathrm{C}(25)-\mathrm{C}(26)-\mathrm{C}(27) & 120.3(3) \\ \mathrm{C}(22)-\mathrm{C}(21)-\mathrm{C}(20) & 120.7(3) & \mathrm{C}(25)-\mathrm{C}(26)-\mathrm{H}(26) & 119.8 \\ \mathrm{C}(22)-\mathrm{C}(21)-\mathrm{H}(21) & 119.6 & \mathrm{C}(27)-\mathrm{C}(26)-\mathrm{H}(26) & 119.8 \\ \mathrm{C}(20)-\mathrm{C}(21)-\mathrm{H}(21) & 119.6 & \mathrm{C}(28)-\mathrm{C}(27)-\mathrm{C}(26) & 119.8(3) \\ \mathrm{C}(21)-\mathrm{C}(22)-\mathrm{C}(23) & 120.3(3) & \mathrm{C}(28)-\mathrm{C}(27)-\mathrm{H}(27) & 120.1 \\ \mathrm{C}(21)-\mathrm{C}(22)-\mathrm{H}(22) & 119.9 & \mathrm{C}(26)-\mathrm{C}(27)-\mathrm{H}(27) & 120.1 \\ \mathrm{C}(23)-\mathrm{C}(22)-\mathrm{H}(22) & 119.9 & \mathrm{C}(29)-\mathrm{C}(28)-\mathrm{C}(27) & 120.6(2) \\ \mathrm{C}(22)-\mathrm{C}(23)-\mathrm{C}(24) & 119.8(3) & \mathrm{C}(29)-\mathrm{C}(28)-\mathrm{H}(28) & 119.7 \\ \mathrm{C}(22)-\mathrm{C}(23)-\mathrm{H}(23) & 120.1 & \mathrm{C}(27)-\mathrm{C}(28)-\mathrm{H}(28) & 119.7 \\ \mathrm{C}(24)-\mathrm{C}(23)-\mathrm{H}(23) & 120.1 & \mathrm{C}(28)-\mathrm{C}(29)-\mathrm{C}(30) & 120.2(3) \\ \mathrm{C}(19)-\mathrm{C}(24)-\mathrm{C}(23) & 120.1(3) & \mathrm{C}(28)-\mathrm{C}(29)-\mathrm{H}(29) & 119.9 \\ \mathrm{C}(19)-\mathrm{C}(24)-\mathrm{H}(24) & 120.0 & \mathrm{C}(30)-\mathrm{C}(29)-\mathrm{H}(29) & 119.9 \\ \mathrm{C}(23)-\mathrm{C}(24)-\mathrm{H}(24) & 120.0 & \mathrm{C}(25)-\mathrm{C}(30)-\mathrm{C}(29) & 119.6(3) \\ \mathrm{C}(26)-\mathrm{C}(25)-\mathrm{C}(30) & 119.4(2) & \mathrm{C}(25)-\mathrm{C}(30)-\mathrm{H}(30) & 120.2 \\ \mathrm{C}(26)-\mathrm{C}(25)-\mathrm{P}(2) & 117.60(18) & \mathrm{C}(29)-\mathrm{C}(30)-\mathrm{H}(30) & 120.2\end{array}$


Table S16. Anisotropic displacement parameters $\left(\AA^{2} \times 10^{3}\right)$ for neijk31. The anisotropic displacement factor exponent takes the form: $-2 \pi^{2}\left[h^{2} a^{* 2} U_{11}+\ldots+2 h k a^{*} b^{*} U_{12}\right]$

\begin{tabular}{|c|c|c|c|c|c|c|}
\hline & $\mathrm{U}_{11}$ & $\mathrm{U}_{22}$ & $\mathrm{U}_{33}$ & $\mathrm{U}_{23}$ & $\mathrm{U}_{13}$ & $\mathrm{U}_{12}$ \\
\hline Fe1 & $36(1)$ & $45(1)$ & $74(1)$ & $-13(1)$ & $4(1)$ & $-11(1)$ \\
\hline Cl1 & $59(1)$ & $63(1)$ & 109(1) & $-12(1)$ & $-28(1)$ & $6(1)$ \\
\hline $\mathrm{Cl} 2$ & $75(1)$ & $74(1)$ & $124(1)$ & $-27(1)$ & $39(1)$ & $-41(1)$ \\
\hline P1 & $37(1)$ & $37(1)$ & $53(1)$ & $-6(1)$ & $1(1)$ & $-11(1)$ \\
\hline $\mathrm{P} 2$ & $43(1)$ & $39(1)$ & $48(1)$ & $-4(1)$ & $0(1)$ & $-11(1)$ \\
\hline $\mathrm{C} 1$ & $39(1)$ & $37(1)$ & $52(1)$ & $-2(1)$ & $0(1)$ & $-12(1)$ \\
\hline $\mathrm{C} 2$ & $64(1)$ & $54(1)$ & $57(1)$ & $-9(1)$ & $11(1)$ & $-27(1)$ \\
\hline $\mathrm{C} 3$ & $72(2)$ & $68(2)$ & $72(2)$ & $-5(1)$ & $18(1)$ & $-39(1)$ \\
\hline $\mathrm{C} 4$ & $76(2)$ & $69(2)$ & $76(2)$ & $-5(1)$ & $3(1)$ & $-47(1)$ \\
\hline C5 & $64(1)$ & $55(1)$ & $58(1)$ & $-7(1)$ & $-3(1)$ & $-30(1)$ \\
\hline C6 & $40(1)$ & $41(1)$ & $49(1)$ & $0(1)$ & $-5(1)$ & $-13(1)$ \\
\hline $\mathrm{C} 7$ & $44(1)$ & $52(1)$ & $57(1)$ & $-6(1)$ & $-1(1)$ & $-20(1)$ \\
\hline $\mathrm{C} 8$ & $54(1)$ & $58(1)$ & $64(1)$ & $-13(1)$ & $-6(1)$ & $-21(1)$ \\
\hline C9 & $70(2)$ & $82(2)$ & $73(2)$ & $-28(2)$ & $-1(1)$ & $-34(2)$ \\
\hline $\mathrm{C} 10$ & $80(2)$ & $106(2)$ & $58(2)$ & $-10(2)$ & $-8(1)$ & $-49(2)$ \\
\hline $\mathrm{C} 11$ & $82(2)$ & $91(2)$ & $65(2)$ & $9(2)$ & $-18(1)$ & $-32(2)$ \\
\hline $\mathrm{C} 12$ & $72(2)$ & $61(2)$ & $66(2)$ & $-1(1)$ & $-12(1)$ & $-18(1)$ \\
\hline $\mathrm{C} 13$ & $41(1)$ & $38(1)$ & $53(1)$ & $-10(1)$ & $-1(1)$ & $-11(1)$ \\
\hline $\mathrm{C} 14$ & $44(1)$ & $52(1)$ & $72(2)$ & $-5(1)$ & $2(1)$ & $-11(1)$ \\
\hline $\mathrm{C} 15$ & $42(1)$ & $59(2)$ & $97(2)$ & $-6(1)$ & $-4(1)$ & $-1(1)$ \\
\hline $\mathrm{C} 16$ & $65(2)$ & $47(1)$ & $92(2)$ & $2(1)$ & $-17(1)$ & $-2(1)$ \\
\hline $\mathrm{C} 17$ & $73(2)$ & $48(1)$ & $73(2)$ & $6(1)$ & $-5(1)$ & $-16(1)$ \\
\hline $\mathrm{C} 18$ & $48(1)$ & $44(1)$ & $64(1)$ & $-7(1)$ & $2(1)$ & $-13(1)$ \\
\hline C19 & $54(1)$ & $42(1)$ & $49(1)$ & $-6(1)$ & $-3(1)$ & $-12(1)$ \\
\hline $\mathrm{C} 20$ & $56(1)$ & $63(2)$ & $62(1)$ & $2(1)$ & $-6(1)$ & $-10(1)$ \\
\hline $\mathrm{C} 21$ & $70(2)$ & $80(2)$ & $85(2)$ & $6(2)$ & $-18(2)$ & $0(2)$ \\
\hline $\mathrm{C} 22$ & $107(3)$ & $70(2)$ & $82(2)$ & $19(2)$ & $-31(2)$ & $-9(2)$ \\
\hline $\mathrm{C} 23$ & $110(3)$ & $80(2)$ & $76(2)$ & $27(2)$ & $-9(2)$ & $-34(2)$ \\
\hline C24 & $67(2)$ & $69(2)$ & $68(2)$ & $14(1)$ & $0(1)$ & $-25(1)$ \\
\hline $\mathrm{C} 25$ & $58(1)$ & $40(1)$ & $49(1)$ & $-6(1)$ & $3(1)$ & $-13(1)$ \\
\hline C26 & $82(2)$ & $49(1)$ & 61(1) & $-6(1)$ & $-9(1)$ & $-6(1)$ \\
\hline
\end{tabular}




\begin{tabular}{lllllll}
$\mathrm{C} 27$ & $112(2)$ & $47(1)$ & $73(2)$ & $-9(1)$ & $-6(2)$ & $0(2)$ \\
$\mathrm{C} 28$ & $136(3)$ & $49(2)$ & $70(2)$ & $-19(1)$ & $10(2)$ & $-19(2)$ \\
$\mathrm{C} 29$ & $128(3)$ & $72(2)$ & $60(2)$ & $-20(1)$ & $-11(2)$ & $-28(2)$ \\
$\mathrm{C} 30$ & $89(2)$ & $56(1)$ & $61(1)$ & $-10(1)$ & $-13(1)$ & $-15(1)$ \\
\hline
\end{tabular}


Table S17. Hydrogen coordinates $\left(\times 10^{4}\right)$ and isotropic displacement parameters $\left(\AA^{2} \times 10^{3}\right)$ for neijk31.

\begin{tabular}{|c|c|c|c|c|}
\hline & $\mathrm{x}$ & $\mathrm{y}$ & z & $\mathrm{U}(\mathrm{eq})$ \\
\hline $\mathrm{H} 2$ & 3629 & 7290 & 211 & 70 \\
\hline H3 & 4965 & 8817 & 213 & 83 \\
\hline $\mathrm{H} 4$ & 4539 & 10158 & 1249 & 83 \\
\hline H5 & 2788 & 9951 & 2299 & 68 \\
\hline H8 & 2662 & 4068 & 319 & 69 \\
\hline H9 & 2470 & 3884 & -965 & 86 \\
\hline H10 & 1091 & 5831 & -1724 & 92 \\
\hline H11 & -133 & 8003 & -1215 & 95 \\
\hline H12 & 60 & 8239 & 60 & 81 \\
\hline H14 & 4698 & 4809 & 1081 & 70 \\
\hline H15 & 6317 & 2670 & 1619 & 85 \\
\hline H16 & 5369 & 1373 & 2611 & 87 \\
\hline H17 & 2771 & 2179 & 3063 & 81 \\
\hline H18 & 1111 & 4288 & 2501 & 64 \\
\hline $\mathrm{H} 20$ & 4069 & 6814 & 2915 & 77 \\
\hline $\mathrm{H} 21$ & 5408 & 4987 & 3772 & 103 \\
\hline $\mathrm{H} 22$ & 4115 & 3963 & 4744 & 112 \\
\hline $\mathrm{H} 23$ & 1454 & 4734 & 4873 & 110 \\
\hline $\mathrm{H} 24$ & 76 & 6557 & 4010 & 85 \\
\hline $\mathrm{H} 26$ & -1373 & 10976 & 2435 & 82 \\
\hline $\mathrm{H} 27$ & -2185 & 13144 & 2970 & 102 \\
\hline $\mathrm{H} 28$ & -1142 & 13329 & 4045 & 107 \\
\hline H29 & 733 & 11391 & 4582 & 104 \\
\hline H30 & 1590 & 9210 & 4045 & 84 \\
\hline
\end{tabular}


Table S18. Torsion angles $\left[{ }^{\circ}\right]$ for neijk 31 .

\begin{tabular}{|c|c|c|c|}
\hline C13-P1-C1-C2 & $87.52(19)$ & $\mathrm{C} 10-\mathrm{C} 11-\mathrm{C} 12-\mathrm{C} 7$ & $-0.2(4)$ \\
\hline C7-P1-C1-C2 & $-23.9(2)$ & C8-C7-C12-C11 & $-0.7(4)$ \\
\hline Fe1-P1-C1-C2 & $-150.06(17)$ & $\mathrm{P} 1-\mathrm{C} 7-\mathrm{C} 12-\mathrm{C} 11$ & $-176.9(2)$ \\
\hline C13-P1-C1-C6 & $-91.85(16)$ & C7-P1-C13-C18 & $-116.57(18)$ \\
\hline C7-P1-C1-C6 & $156.71(16)$ & C1-P1-C13-C18 & $133.92(18)$ \\
\hline Fe1-P1-C1-C6 & $30.57(16)$ & Fe1-P1-C13-C18 & $22.3(2)$ \\
\hline $\mathrm{C} 6-\mathrm{C} 1-\mathrm{C} 2-\mathrm{C} 3$ & $1.5(3)$ & C7-P1-C13-C14 & $64.5(2)$ \\
\hline P1-C1-C2-C3 & $-177.86(19)$ & C1-P1-C13-C14 & $-45.0(2)$ \\
\hline $\mathrm{C} 1-\mathrm{C} 2-\mathrm{C} 3-\mathrm{C} 4$ & $-1.4(4)$ & Fe1-P1-C13-C14 & $-156.64(16)$ \\
\hline $\mathrm{C} 2-\mathrm{C} 3-\mathrm{C} 4-\mathrm{C} 5$ & $0.4(4)$ & C18-C13-C14-C15 & $1.2(4)$ \\
\hline $\mathrm{C} 3-\mathrm{C} 4-\mathrm{C} 5-\mathrm{C} 6$ & $0.6(4)$ & $\mathrm{P} 1-\mathrm{C} 13-\mathrm{C} 14-\mathrm{C} 15$ & $-179.83(19)$ \\
\hline C4-C5-C6-C1 & $-0.6(3)$ & C13-C14-C15-C16 & $-1.5(4)$ \\
\hline C4-C5-C6-P2 & $179.68(19)$ & $\mathrm{C} 14-\mathrm{C} 15-\mathrm{C} 16-\mathrm{C} 17$ & $0.6(4)$ \\
\hline C2-C1-C6-C5 & $-0.5(3)$ & C15-C16-C17-C18 & $0.6(4)$ \\
\hline $\mathrm{P} 1-\mathrm{C} 1-\mathrm{C} 6-\mathrm{C} 5$ & $178.89(16)$ & $\mathrm{C} 14-\mathrm{C} 13-\mathrm{C} 18-\mathrm{C} 17$ & $-0.1(3)$ \\
\hline C2-C1-C6-P2 & $179.26(16)$ & $\mathrm{P} 1-\mathrm{C} 13-\mathrm{C} 18-\mathrm{C} 17$ & $-179.06(18)$ \\
\hline P1-C1-C6-P2 & $-1.3(2)$ & $\mathrm{C} 16-\mathrm{C} 17-\mathrm{C} 18-\mathrm{C} 13$ & $-0.8(4)$ \\
\hline C19-P2-C6-C5 & $-88.77(19)$ & $\mathrm{C} 25-\mathrm{P} 2-\mathrm{C} 19-\mathrm{C} 20$ & $-97.5(2)$ \\
\hline C25-P2-C6-C5 & $22.6(2)$ & C6-P2-C19-C20 & $13.0(2)$ \\
\hline Fe1-P2-C6-C5 & $151.04(17)$ & Fe1-P2-C19-C20 & $124.98(19)$ \\
\hline C19-P2-C6-C1 & $91.47(17)$ & C25-P2-C19-C24 & $84.6(2)$ \\
\hline C25-P2-C6-C1 & $-157.14(16)$ & C6-P2-C19-C24 & $-164.79(19)$ \\
\hline Fe1-P2-C6-C1 & $-28.72(16)$ & Fe1-P2-C19-C24 & $-52.9(2)$ \\
\hline C13-P1-C7-C12 & $-175.25(18)$ & C24-C19-C20-C21 & $-0.1(4)$ \\
\hline C1-P1-C7-C12 & $-66.0(2)$ & P2-C19-C20-C21 & $-178.0(2)$ \\
\hline $\mathrm{Fe} 1-\mathrm{P} 1-\mathrm{C} 7-\mathrm{C} 12$ & $47.5(2)$ & C19-C20-C21-C22 & $-0.2(5)$ \\
\hline C13-P1-C7-C8 & $8.7(2)$ & $\mathrm{C} 20-\mathrm{C} 21-\mathrm{C} 22-\mathrm{C} 23$ & $0.3(5)$ \\
\hline $\mathrm{C} 1-\mathrm{P} 1-\mathrm{C} 7-\mathrm{C} 8$ & $117.94(19)$ & $\mathrm{C} 21-\mathrm{C} 22-\mathrm{C} 23-\mathrm{C} 24$ & $0.1(5)$ \\
\hline Fe1-P1-C7-C8 & $-128.57(17)$ & C20-C19-C24-C23 & $0.5(4)$ \\
\hline C12-C7-C8-C9 & $1.2(3)$ & $\mathrm{P} 2-\mathrm{C} 19-\mathrm{C} 24-\mathrm{C} 23$ & $178.4(2)$ \\
\hline P1-C7-C8-C9 & $177.18(18)$ & $\mathrm{C} 22-\mathrm{C} 23-\mathrm{C} 24-\mathrm{C} 19$ & $-0.4(5)$ \\
\hline $\mathrm{C} 7-\mathrm{C} 8-\mathrm{C} 9-\mathrm{C} 10$ & $-0.8(4)$ & C19-P2-C25-C26 & $-167.13(19)$ \\
\hline C8-C9-C10-C11 & $-0.2(4)$ & C6-P2-C25-C26 & $81.8(2)$ \\
\hline C9-C10-C11-C12 & $0.7(5)$ & Fe1-P2-C25-C26 & $-33.6(2)$ \\
\hline
\end{tabular}




\begin{tabular}{lclr}
$\mathrm{C} 19-\mathrm{P} 2-\mathrm{C} 25-\mathrm{C} 30$ & $12.8(2)$ & $\mathrm{C} 26-\mathrm{C} 27-\mathrm{C} 28-\mathrm{C} 29$ & $0.5(5)$ \\
$\mathrm{C} 6-\mathrm{P} 2-\mathrm{C} 25-\mathrm{C} 30$ & $-98.3(2)$ & $\mathrm{C} 27-\mathrm{C} 28-\mathrm{C} 29-\mathrm{C} 30$ & $-0.1(5)$ \\
$\mathrm{Fe} 1-\mathrm{P} 2-\mathrm{C} 25-\mathrm{C} 30$ & $146.24(19)$ & $\mathrm{C} 26-\mathrm{C} 25-\mathrm{C} 30-\mathrm{C} 29$ & $-0.9(4)$ \\
$\mathrm{C} 30-\mathrm{C} 25-\mathrm{C} 26-\mathrm{C} 27$ & $1.3(4)$ & $\mathrm{P} 2-\mathrm{C} 25-\mathrm{C} 30-\mathrm{C} 29$ & $179.3(2)$ \\
$\mathrm{P} 2-\mathrm{C} 25-\mathrm{C} 26-\mathrm{C} 27$ & $-178.8(2)$ & $\mathrm{C} 28-\mathrm{C} 29-\mathrm{C} 30-\mathrm{C} 25$ & $0.2(5)$ \\
$\mathrm{C} 25-\mathrm{C} 26-\mathrm{C} 27-\mathrm{C} 28$ & $-1.1(5)$ & & \\
& & & \\
\hline
\end{tabular}




\section{4 $\mathrm{FeCl}_{2}$ (Xantphos)}

REFERENCE NUMBER: neivf02

CRYSTAL STRUCTURE REPORT

$\mathrm{C}_{39} \mathrm{H}_{32} \mathrm{Cl}_{2} \mathrm{Fe} \mathrm{O} \mathrm{P}_{2}$

or

$\mathrm{FeCl}_{2}$ (xantphos)

Report prepared for:

V. Fleischauer, Prof. M. Neidig

March 10, 2014

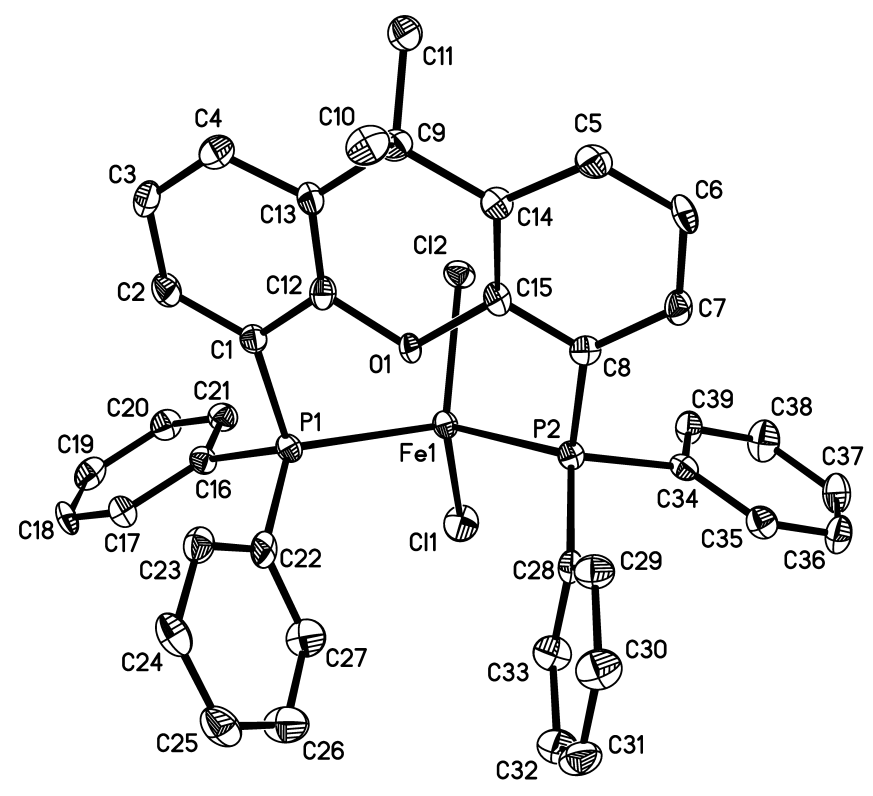

William W. Brennessel

X-ray Crystallographic Facility

Department of Chemistry, University of Rochester

120 Trustee Road

Rochester, NY 14627 


\section{Data collection}

A crystal $\left(0.26 \times 0.12 \times 0.06 \mathrm{~mm}^{3}\right)$ was placed onto the tip of a $0.1 \mathrm{~mm}$ diameter glass capillary tube or fiber and mounted on a Bruker SMART APEX II CCD platform diffractometer for a data collection at 100.0(5) $\mathrm{K}^{1} \mathrm{~A}$ preliminary set of cell constants and an orientation matrix were calculated from reflections harvested from three orthogonal wedges of reciprocal space. The full data collection was carried out using MoK $\alpha$ radiation (graphite monochromator) with a frame time of 90 seconds and a detector distance of $5.03 \mathrm{~cm}$. A randomly oriented region of reciprocal space was surveyed: six major sections of frames were collected with $0.50^{\circ}$ steps in $\omega$ at six different $\phi$ settings and a detector position of $-33^{\circ}$ in $2 \theta$. The intensity data were corrected for absorption. ${ }^{2}$ Final cell constants were calculated from the xyz centroids of 4070 strong reflections from the actual data collection after integration. ${ }^{3}$ See Table 1 for additional crystal and refinement information.

\section{Structure solution and refinement}

The structure was solved using SIR2011 4 and refined using SHELXL-2014. ${ }^{5}$ The space group $P 2{ }_{1} / n$ was determined based on systematic absences. A direct-methods solution was calculated which provided most nonhydrogen atoms from the E-map. Full-matrix least squares / difference Fourier cycles were performed which located the remaining non-hydrogen atoms. All non-hydrogen atoms were refined with anisotropic displacement parameters. All hydrogen atoms were placed in ideal positions and refined as riding atoms with relative isotropic displacement parameters. The final full matrix least squares refinement converged to $R 1=0.0581\left(F^{2}, I>2 \sigma(I)\right)$ and $w R 2=0.1388\left(F^{2}\right.$, all data).

\section{Structure description}

The structure is the one suggested. The asymmetric unit contains two independent molecules in general positions.

Unless noted otherwise all structural diagrams containing thermal displacement ellipsoids are drawn at the $50 \%$ probability level.

Data collection, structure solution, and structure refinement were conducted at the X-ray Crystallographic Facility, B51 Hutchison Hall, Department of Chemistry, University of Rochester. All publications arising from this report MUST either 1) include William W. Brennessel as a coauthor or 2) acknowledge William W. Brennessel and the Xray Crystallographic Facility of the Department of Chemistry at the University of Rochester. 
1 APEX2, version 2013.10-0; Bruker AXS: Madison, WI, 2013.

2 Sheldrick, G. M. SADABS, version 2012/1; University of Göttingen: Göttingen, Germany, 2012.

3 SAINT, version 8.34A; Bruker AXS: Madison, WI, 2013.

4 Burla, M. C.; Caliandro, R.; Camalli, M.; Carrozzini, B.; Cascarano, G. L.; Giacovazzo, C.; Mallamo, M.;

Mazzone, A.; Polidori, G.; Spagna, R. SIR2011: a new package for crystal structure determination and refinement, version 1.0; Istituto di Cristallografia: Bari, Italy, 2012.

5 Sheldrick, G. M. SHELXL-2014/1; University of Göttingen: Göttingen, Germany, 2014.

Some equations of interest:

$$
\begin{gathered}
R_{\mathrm{int}}=\Sigma\left|F_{\mathrm{o}}{ }^{2}-<F_{\mathrm{o}}{ }^{2}>\right| / \Sigma\left|F_{\mathrm{o}}{ }^{2}\right| \\
R 1=\Sigma|| F_{\mathrm{o}}|-| F_{\mathrm{c}}|/ \Sigma| F_{\mathrm{o}} \mid \\
w R 2=\left[\Sigma\left[w\left(F_{\mathrm{o}}{ }^{2}-F_{\mathrm{c}}{ }^{2}\right)^{2}\right] / \Sigma\left[w\left(F_{\mathrm{o}}{ }^{2}\right)^{2}\right]\right]^{1 / 2} \\
\text { where } w=1 /\left[\sigma^{2}\left(F_{\mathrm{o}}{ }^{2}\right)+(a P)^{2}+b P\right] \text { and } \\
P=1 / 3 \max \left(0, F_{\mathrm{o}}{ }^{2}\right)+2 / 3 F_{\mathrm{c}}{ }^{2} \\
\text { GOF }=S=\left[\Sigma\left[w\left(F_{\mathrm{o}}{ }^{2}-F_{\mathrm{c}}{ }^{2}\right)^{2}\right] /(m-n)\right]^{1 / 2}
\end{gathered}
$$

where $m=$ number of reflections and $n=$ number of parameters

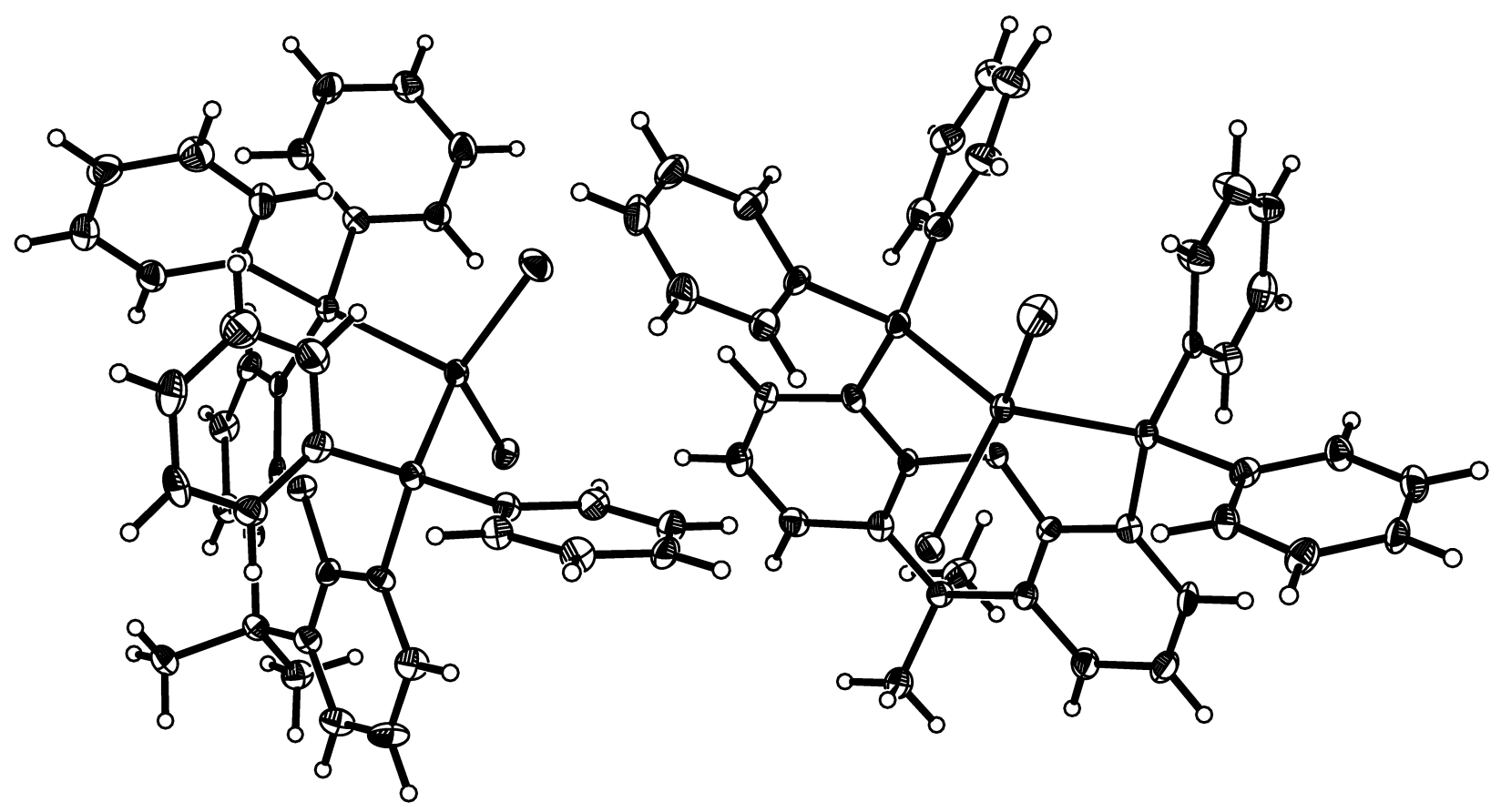



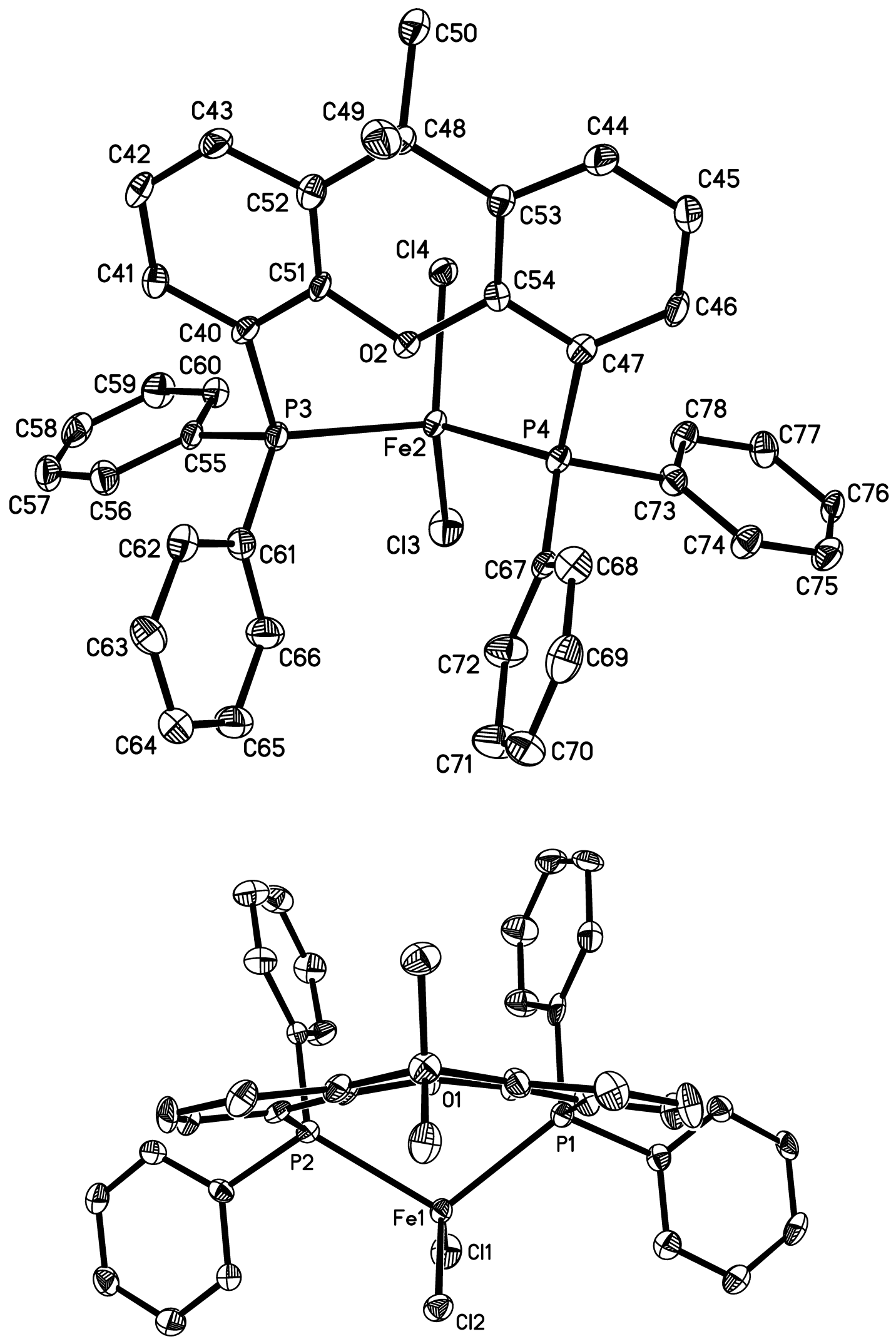
Table S19. Crystal data and structure refinement for neivf02.

\begin{tabular}{|c|c|c|}
\hline Identification code & \multicolumn{2}{|l|}{ neivf02 } \\
\hline Empirical formula & \multicolumn{2}{|c|}{$\mathrm{C} 39 \mathrm{H} 32 \mathrm{C} 12 \mathrm{Fe} \mathrm{O} \mathrm{P} 2$} \\
\hline Formula weight & \multicolumn{2}{|l|}{705.33} \\
\hline Temperature & \multicolumn{2}{|l|}{$100.0(5) \mathrm{K}$} \\
\hline Wavelength & \multicolumn{2}{|l|}{$0.71073 \AA$} \\
\hline Crystal system & \multicolumn{2}{|l|}{ monoclinic } \\
\hline Space group & \multicolumn{2}{|l|}{$P 2_{1} / n$} \\
\hline \multirow[t]{3}{*}{ Unit cell dimensions } & $a=16.882(4) \AA$ & $\alpha=90^{\circ}$ \\
\hline & $b=10.534(3) \AA$ & $\beta=93.412(4)^{\circ}$ \\
\hline & $c=37.753(9) \AA$ & $\gamma=90^{\circ}$ \\
\hline Volume & \multicolumn{2}{|l|}{$6702(3) \AA^{3}$} \\
\hline$Z$ & \multicolumn{2}{|l|}{8} \\
\hline Density (calculated) & \multicolumn{2}{|l|}{$1.398 \mathrm{Mg} / \mathrm{m}^{3}$} \\
\hline Absorption coefficient & \multicolumn{2}{|l|}{$0.736 \mathrm{~mm}^{-1}$} \\
\hline$F(000)$ & \multicolumn{2}{|l|}{2912} \\
\hline Crystal color, morphology & \multicolumn{2}{|l|}{ colorless, plate } \\
\hline Crystal size & \multicolumn{2}{|c|}{$0.26 \times 0.12 \times 0.06 \mathrm{~mm}^{3}$} \\
\hline Theta range for data collection & \multicolumn{2}{|l|}{1.963 to $27.519^{\circ}$} \\
\hline Index ranges & \multicolumn{2}{|c|}{$-21 \leq h \leq 21,-13 \leq k \leq 13,-49 \leq l \leq 49$} \\
\hline Reflections collected & \multicolumn{2}{|l|}{121316} \\
\hline Independent reflections & \multicolumn{2}{|c|}{$15389[R(\mathrm{int})=0.1290]$} \\
\hline Observed reflections & \multicolumn{2}{|l|}{8852} \\
\hline Completeness to theta $=27.485^{\circ}$ & \multicolumn{2}{|l|}{$100.0 \%$} \\
\hline Absorption correction & \multicolumn{2}{|l|}{ Multi-scan } \\
\hline Max. and min. transmission & \multicolumn{2}{|l|}{0.7456 and 0.6259} \\
\hline Refinement method & \multicolumn{2}{|c|}{ Full-matrix least-squares on $F^{2}$} \\
\hline Data / restraints / parameters & \multicolumn{2}{|l|}{$15389 / 0 / 815$} \\
\hline Goodness-of-fit on $F^{2}$ & \multicolumn{2}{|l|}{1.027} \\
\hline Final $R$ indices $[I>2 \operatorname{sigma}(I)]$ & \multicolumn{2}{|c|}{$R 1=0.0581, w R 2=0.1198$} \\
\hline$R$ indices (all data) & \multicolumn{2}{|c|}{$R 1=0.1202, w R 2=0.1388$} \\
\hline Largest diff. peak and hole & \multicolumn{2}{|c|}{0.901 and -0.467 e. $\AA^{-3}$} \\
\hline
\end{tabular}


Table S20. Atomic coordinates $\left(\times 10^{4}\right)$ and equivalent isotropic displacement parameters $\left(\AA^{2} \times 10^{3}\right)$ for neivf02. $U_{e q}$ is defined as one third of the trace of the orthogonalized $U_{i j}$ tensor.

\begin{tabular}{|c|c|c|c|c|}
\hline & $\mathrm{x}$ & $\mathrm{y}$ & $\mathrm{z}$ & $\mathrm{U}_{\mathrm{eq}}$ \\
\hline $\mathrm{Fe} 1$ & $3336(1)$ & $3368(1)$ & 1194(1) & $15(1)$ \\
\hline Cl1 & $3986(1)$ & $2666(1)$ & 741(1) & $27(1)$ \\
\hline $\mathrm{Cl} 2$ & $3142(1)$ & $2153(1)$ & 1673(1) & $19(1)$ \\
\hline P1 & $3987(1)$ & $5322(1)$ & 1416(1) & $15(1)$ \\
\hline $\mathrm{P} 2$ & 1975(1) & $3862(1)$ & $970(1)$ & $14(1)$ \\
\hline $\mathrm{O} 1$ & $2407(1)$ & $5200(2)$ & 1616(1) & $15(1)$ \\
\hline $\mathrm{C} 1$ & $3685(2)$ & $5610(4)$ & 1862(1) & $16(1)$ \\
\hline $\mathrm{C} 2$ & $4200(2)$ & $5867(4)$ & $2156(1)$ & $22(1)$ \\
\hline $\mathrm{C} 3$ & $3910(3)$ & 5984(4) & 2490(1) & $26(1)$ \\
\hline $\mathrm{C} 4$ & $3108(3)$ & $5820(4)$ & 2536(1) & $24(1)$ \\
\hline $\mathrm{C} 5$ & $534(2)$ & 4189(4) & 1962(1) & $21(1)$ \\
\hline C6 & $174(2)$ & $3634(4)$ & 1659(1) & $21(1)$ \\
\hline $\mathrm{C} 7$ & $575(2)$ & $3567(4)$ & 1352(1) & $18(1)$ \\
\hline $\mathrm{C} 8$ & $1344(2)$ & 4064(4) & 1339(1) & $14(1)$ \\
\hline C9 & $1684(2)$ & $5321(4)$ & 2302(1) & $18(1)$ \\
\hline $\mathrm{C} 10$ & $1288(3)$ & 6607(4) & 2364(1) & $24(1)$ \\
\hline $\mathrm{C} 11$ & 1591(2) & $4456(4)$ & $2625(1)$ & $23(1)$ \\
\hline $\mathrm{C} 12$ & $2876(2)$ & $5463(4)$ & 1922(1) & $16(1)$ \\
\hline $\mathrm{C} 13$ & $2567(2)$ & $5530(4)$ & 2251(1) & $17(1)$ \\
\hline $\mathrm{C} 14$ & $1295(2)$ & $4700(4)$ & 1967(1) & $16(1)$ \\
\hline $\mathrm{C} 15$ & $1672(2)$ & $4656(4)$ & $1650(1)$ & $14(1)$ \\
\hline C16 & $5065(2)$ & $5177(4)$ & 1469(1) & $16(1)$ \\
\hline $\mathrm{C} 17$ & $5581(2)$ & 6188(4) & 1436(1) & $19(1)$ \\
\hline $\mathrm{C} 18$ & $6400(2)$ & 5997(4) & 1470(1) & $20(1)$ \\
\hline C19 & $6700(2)$ & $4808(4)$ & 1546(1) & $22(1)$ \\
\hline $\mathrm{C} 20$ & $6188(2)$ & $3789(4)$ & 1582(1) & $22(1)$ \\
\hline $\mathrm{C} 21$ & $5375(2)$ & $3966(4)$ & 1542(1) & $20(1)$ \\
\hline $\mathrm{C} 22$ & $3827(2)$ & 6811(4) & $1176(1)$ & $19(1)$ \\
\hline $\mathrm{C} 23$ & $3737(2)$ & 7968(4) & $1345(1)$ & 21(1) \\
\hline $\mathrm{C} 24$ & $3661(2)$ & 9093(4) & $1148(1)$ & $23(1)$ \\
\hline $\mathrm{C} 25$ & $3655(3)$ & $9036(4)$ & $782(1)$ & $28(1)$ \\
\hline
\end{tabular}




\begin{tabular}{|c|c|c|c|c|}
\hline $\mathrm{C} 26$ & $3737(3)$ & 7891(4) & $615(1)$ & $32(1)$ \\
\hline $\mathrm{C} 27$ & $3823(3)$ & $6783(4)$ & $809(1)$ & $27(1)$ \\
\hline $\mathrm{C} 28$ & $1782(2)$ & $5198(4)$ & $676(1)$ & $16(1)$ \\
\hline C29 & 1167(3) & $6053(4)$ & $713(1)$ & $23(1)$ \\
\hline $\mathrm{C} 30$ & 1027(3) & $7005(4)$ & $465(1)$ & $28(1)$ \\
\hline C31 & 1484(3) & 7106(4) & $176(1)$ & $28(1)$ \\
\hline C32 & 2095(3) & 6254(4) & $135(1)$ & $27(1)$ \\
\hline C33 & $2246(2)$ & $5319(4)$ & $385(1)$ & $23(1)$ \\
\hline $\mathrm{C} 34$ & $1542(2)$ & $2558(4)$ & $704(1)$ & $16(1)$ \\
\hline $\mathrm{C} 35$ & $950(2)$ & 2741(4) & $433(1)$ & $20(1)$ \\
\hline $\mathrm{C} 36$ & $697(2)$ & $1736(4)$ & $220(1)$ & $24(1)$ \\
\hline C37 & $1020(2)$ & $548(4)$ & $273(1)$ & $25(1)$ \\
\hline C38 & $1602(3)$ & $339(4)$ & $542(1)$ & $28(1)$ \\
\hline C39 & $1859(2)$ & $1350(4)$ & $757(1)$ & $20(1)$ \\
\hline $\mathrm{Fe} 2$ & $8354(1)$ & $821(1)$ & $1174(1)$ & $14(1)$ \\
\hline $\mathrm{Cl} 3$ & 8999(1) & $1446(1)$ & $708(1)$ & $29(1)$ \\
\hline $\mathrm{Cl} 4$ & $8165(1)$ & 2042(1) & $1653(1)$ & $19(1)$ \\
\hline P3 & 6981(1) & $334(1)$ & $969(1)$ & $15(1)$ \\
\hline P4 & $9000(1)$ & $-1135(1)$ & $1400(1)$ & $14(1)$ \\
\hline $\mathrm{O} 2$ & $7423(1)$ & $-1032(2)$ & $1608(1)$ & $14(1)$ \\
\hline $\mathrm{C} 40$ & $6357(2)$ & $129(4)$ & $1345(1)$ & $15(1)$ \\
\hline C41 & $5596(2)$ & $642(4)$ & $1358(1)$ & $18(1)$ \\
\hline $\mathrm{C} 42$ & $5202(2)$ & $555(4)$ & $1669(1)$ & $22(1)$ \\
\hline $\mathrm{C} 43$ & $5562(2)$ & $7(4)$ & 1970(1) & $20(1)$ \\
\hline C44 & $8148(2)$ & $-1670(4)$ & $2525(1)$ & $24(1)$ \\
\hline $\mathrm{C} 45$ & $8949(2)$ & $-1833(4)$ & $2472(1)$ & $26(1)$ \\
\hline $\mathrm{C} 46$ & $9229(2)$ & $-1718(4)$ & $2135(1)$ & $20(1)$ \\
\hline $\mathrm{C} 47$ & $8706(2)$ & $-1438(4)$ & $1851(1)$ & $15(1)$ \\
\hline C48 & $6718(2)$ & $-1150(4)$ & $2301(1)$ & $16(1)$ \\
\hline C49 & $6316(2)$ & $-2437(4)$ & $2366(1)$ & $24(1)$ \\
\hline $\mathrm{C} 50$ & $6645(2)$ & $-285(4)$ & $2628(1)$ & $23(1)$ \\
\hline C51 & $6686(2)$ & $-473(4)$ & $1651(1)$ & $14(1)$ \\
\hline C52 & $6324(2)$ & $-531(4)$ & 1970(1) & $17(1)$ \\
\hline C53 & $7600(2)$ & $-1375(4)$ & $2244(1)$ & $16(1)$ \\
\hline C54 & $7902(2)$ & $-1281(4)$ & 1913(1) & $14(1)$ \\
\hline C55 & $6528(2)$ & 1651(4) & $717(1)$ & 19(1) \\
\hline
\end{tabular}




\begin{tabular}{|c|c|c|c|c|}
\hline C56 & $5913(2)$ & $1465(4)$ & $456(1)$ & $23(1)$ \\
\hline C57 & $5586(2)$ & $2508(5)$ & $272(1)$ & $28(1)$ \\
\hline C58 & $5865(3)$ & $3706(4)$ & $344(1)$ & $26(1)$ \\
\hline C59 & 6473(3) & $3905(4)$ & $601(1)$ & $27(1)$ \\
\hline C60 & $6803(2)$ & $2865(4)$ & $784(1)$ & $20(1)$ \\
\hline C61 & $6782(2)$ & $-1006(4)$ & $677(1)$ & $18(1)$ \\
\hline C62 & 6164(2) & $-1860(4)$ & $720(1)$ & $21(1)$ \\
\hline C63 & $6004(2)$ & $-2826(4)$ & $476(1)$ & $23(1)$ \\
\hline C64 & $6461(2)$ & $-2929(4)$ & $186(1)$ & $26(1)$ \\
\hline C65 & 7073(3) & $-2087(4)$ & $134(1)$ & $28(1)$ \\
\hline C66 & $7235(3)$ & $-1135(4)$ & $381(1)$ & $27(1)$ \\
\hline C67 & $8840(2)$ & $-2625(4)$ & $1158(1)$ & $14(1)$ \\
\hline C68 & $8724(2)$ & $-3786(4)$ & $1323(1)$ & $21(1)$ \\
\hline C69 & $8646(2)$ & $-4883(4)$ & $1124(1)$ & $26(1)$ \\
\hline $\mathrm{C} 70$ & $8681(2)$ & $-4842(4)$ & $761(1)$ & $26(1)$ \\
\hline C71 & 8791(3) & $-3701(5)$ & $592(1)$ & $34(1)$ \\
\hline $\mathrm{C} 72$ & $8863(3)$ & $-2583(4)$ & $789(1)$ & $26(1)$ \\
\hline $\mathrm{C} 73$ & $10076(2)$ & $-985(4)$ & $1456(1)$ & $18(1)$ \\
\hline C74 & $10598(2)$ & $-2012(4)$ & $1424(1)$ & $20(1)$ \\
\hline $\mathrm{C} 75$ & $11408(2)$ & $-1802(4)$ & $1462(1)$ & $23(1)$ \\
\hline $\mathrm{C} 76$ & $11704(2)$ & $-592(4)$ & 1531(1) & $21(1)$ \\
\hline $\mathrm{C} 77$ & $11202(2)$ & $415(4)$ & $1564(1)$ & $23(1)$ \\
\hline C78 & $10385(2)$ & $224(4)$ & $1518(1)$ & $18(1)$ \\
\hline
\end{tabular}


Table S21. Bond lengths $[\AA]$ and angles $\left[{ }^{\circ}\right]$ for neivf02.

\begin{tabular}{|c|c|c|c|}
\hline $\mathrm{Fe}(1)-\mathrm{Cl}(1)$ & $2.2137(12)$ & $\mathrm{C}(10)-\mathrm{H}(10 \mathrm{C})$ & 0.9800 \\
\hline $\mathrm{Fe}(1)-\mathrm{Cl}(2)$ & $2.2548(12)$ & $\mathrm{C}(11)-\mathrm{H}(11 \mathrm{~A})$ & 0.9800 \\
\hline $\mathrm{Fe}(1)-\mathrm{P}(1)$ & $2.4564(13)$ & $\mathrm{C}(11)-\mathrm{H}(11 \mathrm{~B})$ & 0.9800 \\
\hline $\mathrm{Fe}(1)-\mathrm{P}(2)$ & $2.4572(13)$ & $\mathrm{C}(11)-\mathrm{H}(11 \mathrm{C})$ & 0.9800 \\
\hline $\mathrm{P}(1)-\mathrm{C}(1)$ & $1.814(4)$ & $C(12)-C(13)$ & $1.377(5)$ \\
\hline $\mathrm{P}(1)-\mathrm{C}(22)$ & $1.823(4)$ & $C(14)-C(15)$ & $1.389(5)$ \\
\hline $\mathrm{P}(1)-\mathrm{C}(16)$ & $1.825(4)$ & $C(16)-C(17)$ & $1.385(5)$ \\
\hline $\mathrm{P}(2)-\mathrm{C}(28)$ & $1.811(4)$ & $C(16)-C(21)$ & $1.400(5)$ \\
\hline $\mathrm{P}(2)-\mathrm{C}(8)$ & $1.817(4)$ & $C(17)-C(18)$ & $1.396(5)$ \\
\hline $\mathrm{P}(2)-\mathrm{C}(34)$ & $1.827(4)$ & $\mathrm{C}(17)-\mathrm{H}(17)$ & 0.9500 \\
\hline $\mathrm{O}(1)-\mathrm{C}(15)$ & $1.381(4)$ & $C(18)-C(19)$ & $1.375(6)$ \\
\hline $\mathrm{O}(1)-\mathrm{C}(12)$ & $1.391(4)$ & $\mathrm{C}(18)-\mathrm{H}(18)$ & 0.9500 \\
\hline$C(1)-C(2)$ & $1.395(5)$ & $C(19)-C(20)$ & $1.390(6)$ \\
\hline$C(1)-C(12)$ & $1.406(5)$ & $\mathrm{C}(19)-\mathrm{H}(19)$ & 0.9500 \\
\hline$C(2)-C(3)$ & $1.384(5)$ & $C(20)-C(21)$ & $1.384(5)$ \\
\hline $\mathrm{C}(2)-\mathrm{H}(2)$ & 0.9500 & $\mathrm{C}(20)-\mathrm{H}(20)$ & 0.9500 \\
\hline$C(3)-C(4)$ & $1.386(6)$ & $\mathrm{C}(21)-\mathrm{H}(21)$ & 0.9500 \\
\hline $\mathrm{C}(3)-\mathrm{H}(3)$ & 0.9500 & $C(22)-C(27)$ & $1.386(6)$ \\
\hline$C(4)-C(13)$ & $1.404(5)$ & $C(22)-C(23)$ & $1.387(6)$ \\
\hline $\mathrm{C}(4)-\mathrm{H}(4)$ & 0.9500 & $C(23)-C(24)$ & $1.401(6)$ \\
\hline$C(5)-C(6)$ & $1.391(6)$ & $\mathrm{C}(23)-\mathrm{H}(23)$ & 0.9500 \\
\hline$C(5)-C(14)$ & $1.392(5)$ & $C(24)-C(25)$ & $1.384(6)$ \\
\hline $\mathrm{C}(5)-\mathrm{H}(5)$ & 0.9500 & $\mathrm{C}(24)-\mathrm{H}(24)$ & 0.9500 \\
\hline$C(6)-C(7)$ & $1.381(5)$ & $C(25)-C(26)$ & $1.372(6)$ \\
\hline $\mathrm{C}(6)-\mathrm{H}(6)$ & 0.9500 & $\mathrm{C}(25)-\mathrm{H}(25)$ & 0.9500 \\
\hline$C(7)-C(8)$ & $1.403(5)$ & $C(26)-C(27)$ & $1.382(6)$ \\
\hline $\mathrm{C}(7)-\mathrm{H}(7)$ & 0.9500 & $\mathrm{C}(26)-\mathrm{H}(26)$ & 0.9500 \\
\hline$C(8)-C(15)$ & $1.413(5)$ & $\mathrm{C}(27)-\mathrm{H}(27)$ & 0.9500 \\
\hline$C(9)-C(13)$ & $1.530(5)$ & C(28)-C(29) & $1.388(5)$ \\
\hline C(9)-C(10) & $1.535(5)$ & $C(28)-C(33)$ & $1.392(5)$ \\
\hline C(9)-C(11) & $1.536(5)$ & C(29)-C(30) & $1.382(6)$ \\
\hline$C(9)-C(14)$ & $1.536(5)$ & C(29)-H(29) & 0.9500 \\
\hline $\mathrm{C}(10)-\mathrm{H}(10 \mathrm{~A})$ & 0.9800 & $C(30)-C(31)$ & $1.379(6)$ \\
\hline $\mathrm{C}(10)-\mathrm{H}(10 \mathrm{~B})$ & 0.9800 & $\mathrm{C}(30)-\mathrm{H}(30)$ & 0.9500 \\
\hline
\end{tabular}




\begin{tabular}{|c|c|c|c|}
\hline$C(31)-C(32)$ & $1.383(6)$ & $\mathrm{C}(44)-\mathrm{C}(45)$ & $1.391(6)$ \\
\hline $\mathrm{C}(31)-\mathrm{H}(31)$ & 0.9500 & $\mathrm{C}(44)-\mathrm{C}(53)$ & $1.401(5)$ \\
\hline$C(32)-C(33)$ & $1.378(6)$ & $\mathrm{C}(44)-\mathrm{H}(44)$ & 0.9500 \\
\hline $\mathrm{C}(32)-\mathrm{H}(32)$ & 0.9500 & $C(45)-C(46)$ & $1.392(5)$ \\
\hline $\mathrm{C}(33)-\mathrm{H}(33)$ & 0.9500 & $\mathrm{C}(45)-\mathrm{H}(45)$ & 0.9500 \\
\hline$C(34)-C(39)$ & $1.390(5)$ & $C(46)-C(47)$ & $1.379(5)$ \\
\hline$C(34)-C(35)$ & $1.401(5)$ & $\mathrm{C}(46)-\mathrm{H}(46)$ & 0.9500 \\
\hline$C(35)-C(36)$ & $1.383(6)$ & $C(47)-C(54)$ & $1.401(5)$ \\
\hline $\mathrm{C}(35)-\mathrm{H}(35)$ & 0.9500 & $\mathrm{C}(48)-\mathrm{C}(52)$ & $1.529(5)$ \\
\hline$C(36)-C(37)$ & $1.376(6)$ & $C(48)-C(53)$ & $1.536(5)$ \\
\hline $\mathrm{C}(36)-\mathrm{H}(36)$ & 0.9500 & $\mathrm{C}(48)-\mathrm{C}(49)$ & $1.542(5)$ \\
\hline $\mathrm{C}(37)-\mathrm{C}(38)$ & $1.388(6)$ & $\mathrm{C}(48)-\mathrm{C}(50)$ & $1.543(5)$ \\
\hline $\mathrm{C}(37)-\mathrm{H}(37)$ & 0.9500 & $\mathrm{C}(49)-\mathrm{H}(49 \mathrm{~A})$ & 0.9800 \\
\hline $\mathrm{C}(38)-\mathrm{C}(39)$ & $1.392(6)$ & $\mathrm{C}(49)-\mathrm{H}(49 \mathrm{~B})$ & 0.9800 \\
\hline $\mathrm{C}(38)-\mathrm{H}(38)$ & 0.9500 & $\mathrm{C}(49)-\mathrm{H}(49 \mathrm{C})$ & 0.9800 \\
\hline $\mathrm{C}(39)-\mathrm{H}(39)$ & 0.9500 & $\mathrm{C}(50)-\mathrm{H}(50 \mathrm{~A})$ & 0.9800 \\
\hline $\mathrm{Fe}(2)-\mathrm{Cl}(3)$ & $2.2247(12)$ & $\mathrm{C}(50)-\mathrm{H}(50 \mathrm{~B})$ & 0.9800 \\
\hline $\mathrm{Fe}(2)-\mathrm{Cl}(4)$ & $2.2565(12)$ & $\mathrm{C}(50)-\mathrm{H}(50 \mathrm{C})$ & 0.9800 \\
\hline $\mathrm{Fe}(2)-\mathrm{P}(3)$ & $2.4543(13)$ & $C(51)-C(52)$ & $1.383(5)$ \\
\hline $\mathrm{Fe}(2)-\mathrm{P}(4)$ & $2.4595(13)$ & $\mathrm{C}(53)-\mathrm{C}(54)$ & $1.382(5)$ \\
\hline $\mathrm{P}(3)-\mathrm{C}(61)$ & $1.811(4)$ & $C(55)-C(60)$ & $1.380(6)$ \\
\hline $\mathrm{P}(3)-\mathrm{C}(55)$ & $1.824(4)$ & $C(55)-C(56)$ & $1.401(5)$ \\
\hline $\mathrm{P}(3)-\mathrm{C}(40)$ & $1.831(4)$ & $C(56)-C(57)$ & $1.396(6)$ \\
\hline$P(4)-C(73)$ & $1.822(4)$ & $\mathrm{C}(56)-\mathrm{H}(56)$ & 0.9500 \\
\hline $\mathrm{P}(4)-\mathrm{C}(67)$ & $1.827(4)$ & $\mathrm{C}(57)-\mathrm{C}(58)$ & $1.368(6)$ \\
\hline$P(4)-C(47)$ & $1.831(4)$ & $\mathrm{C}(57)-\mathrm{H}(57)$ & 0.9500 \\
\hline $\mathrm{O}(2)-\mathrm{C}(54)$ & $1.390(4)$ & $\mathrm{C}(58)-\mathrm{C}(59)$ & $1.384(6)$ \\
\hline $\mathrm{O}(2)-\mathrm{C}(51)$ & $1.395(4)$ & $\mathrm{C}(58)-\mathrm{H}(58)$ & 0.9500 \\
\hline $\mathrm{C}(40)-\mathrm{C}(41)$ & $1.397(5)$ & $C(59)-C(60)$ & $1.395(6)$ \\
\hline$C(40)-C(51)$ & $1.401(5)$ & $\mathrm{C}(59)-\mathrm{H}(59)$ & 0.9500 \\
\hline$C(41)-C(42)$ & $1.388(5)$ & $\mathrm{C}(60)-\mathrm{H}(60)$ & 0.9500 \\
\hline $\mathrm{C}(41)-\mathrm{H}(41)$ & 0.9500 & $\mathrm{C}(61)-\mathrm{C}(62)$ & $1.395(5)$ \\
\hline$C(42)-C(43)$ & $1.380(6)$ & $\mathrm{C}(61)-\mathrm{C}(66)$ & $1.398(5)$ \\
\hline $\mathrm{C}(42)-\mathrm{H}(42)$ & 0.9500 & $C(62)-C(63)$ & $1.389(6)$ \\
\hline $\mathrm{C}(43)-\mathrm{C}(52)$ & $1.406(5)$ & $\mathrm{C}(62)-\mathrm{H}(62)$ & 0.9500 \\
\hline $\mathrm{C}(43)-\mathrm{H}(43)$ & 0.9500 & $\mathrm{C}(63)-\mathrm{C}(64)$ & $1.383(6)$ \\
\hline
\end{tabular}




\begin{tabular}{|c|c|c|c|}
\hline $\mathrm{C}(63)-\mathrm{H}(63)$ & 0.9500 & $\mathrm{C}(22)-\mathrm{P}(1)-\mathrm{C}(16)$ & $104.12(18)$ \\
\hline$C(64)-C(65)$ & $1.384(6)$ & $\mathrm{C}(1)-\mathrm{P}(1)-\mathrm{Fe}(1)$ & $108.13(13)$ \\
\hline $\mathrm{C}(64)-\mathrm{H}(64)$ & 0.9500 & $\mathrm{C}(22)-\mathrm{P}(1)-\mathrm{Fe}(1)$ & $120.12(14)$ \\
\hline$C(65)-C(66)$ & $1.385(6)$ & $\mathrm{C}(16)-\mathrm{P}(1)-\mathrm{Fe}(1)$ & $112.97(13)$ \\
\hline $\mathrm{C}(65)-\mathrm{H}(65)$ & 0.9500 & $\mathrm{C}(28)-\mathrm{P}(2)-\mathrm{C}(8)$ & $106.72(18)$ \\
\hline C(66)-H(66) & 0.9500 & $\mathrm{C}(28)-\mathrm{P}(2)-\mathrm{C}(34)$ & $101.32(18)$ \\
\hline $\mathrm{C}(67)-\mathrm{C}(68)$ & $1.390(5)$ & $\mathrm{C}(8)-\mathrm{P}(2)-\mathrm{C}(34)$ & $106.02(18)$ \\
\hline$C(67)-C(72)$ & $1.397(5)$ & $\mathrm{C}(28)-\mathrm{P}(2)-\mathrm{Fe}(1)$ & $120.42(13)$ \\
\hline $\mathrm{C}(68)-\mathrm{C}(69)$ & $1.381(6)$ & $\mathrm{C}(8)-\mathrm{P}(2)-\mathrm{Fe}(1)$ & $109.82(13)$ \\
\hline C(68)-H(68) & 0.9500 & $\mathrm{C}(34)-\mathrm{P}(2)-\mathrm{Fe}(1)$ & $111.42(13)$ \\
\hline $\mathrm{C}(69)-\mathrm{C}(70)$ & $1.374(6)$ & $\mathrm{C}(15)-\mathrm{O}(1)-\mathrm{C}(12)$ & 118.3(3) \\
\hline C(69)-H(69) & 0.9500 & $\mathrm{C}(2)-\mathrm{C}(1)-\mathrm{C}(12)$ & $117.2(4)$ \\
\hline$C(70)-C(71)$ & $1.378(6)$ & $\mathrm{C}(2)-\mathrm{C}(1)-\mathrm{P}(1)$ & $125.0(3)$ \\
\hline $\mathrm{C}(70)-\mathrm{H}(70)$ & 0.9500 & $\mathrm{C}(12)-\mathrm{C}(1)-\mathrm{P}(1)$ & $117.5(3)$ \\
\hline $\mathrm{C}(71)-\mathrm{C}(72)$ & $1.394(6)$ & $C(3)-C(2)-C(1)$ & $120.3(4)$ \\
\hline $\mathrm{C}(71)-\mathrm{H}(71)$ & 0.9500 & $\mathrm{C}(3)-\mathrm{C}(2)-\mathrm{H}(2)$ & 119.9 \\
\hline $\mathrm{C}(72)-\mathrm{H}(72)$ & 0.9500 & $\mathrm{C}(1)-\mathrm{C}(2)-\mathrm{H}(2)$ & 119.9 \\
\hline $\mathrm{C}(73)-\mathrm{C}(78)$ & $1.392(5)$ & $\mathrm{C}(2)-\mathrm{C}(3)-\mathrm{C}(4)$ & $120.4(4)$ \\
\hline$C(73)-C(74)$ & $1.405(5)$ & $\mathrm{C}(2)-\mathrm{C}(3)-\mathrm{H}(3)$ & 119.8 \\
\hline$C(74)-C(75)$ & $1.385(5)$ & $\mathrm{C}(4)-\mathrm{C}(3)-\mathrm{H}(3)$ & 119.8 \\
\hline $\mathrm{C}(74)-\mathrm{H}(74)$ & 0.9500 & $C(3)-C(4)-C(13)$ & $121.7(4)$ \\
\hline$C(75)-C(76)$ & $1.388(6)$ & $\mathrm{C}(3)-\mathrm{C}(4)-\mathrm{H}(4)$ & 119.2 \\
\hline $\mathrm{C}(75)-\mathrm{H}(75)$ & 0.9500 & $\mathrm{C}(13)-\mathrm{C}(4)-\mathrm{H}(4)$ & 119.2 \\
\hline$C(76)-C(77)$ & $1.369(5)$ & $C(6)-C(5)-C(14)$ & $122.1(4)$ \\
\hline $\mathrm{C}(76)-\mathrm{H}(76)$ & 0.9500 & $\mathrm{C}(6)-\mathrm{C}(5)-\mathrm{H}(5)$ & 118.9 \\
\hline C(77)-C(78) & $1.393(5)$ & $\mathrm{C}(14)-\mathrm{C}(5)-\mathrm{H}(5)$ & 118.9 \\
\hline $\mathrm{C}(77)-\mathrm{H}(77)$ & 0.9500 & $C(7)-C(6)-C(5)$ & $120.0(4)$ \\
\hline $\mathrm{C}(78)-\mathrm{H}(78)$ & 0.9500 & $\mathrm{C}(7)-\mathrm{C}(6)-\mathrm{H}(6)$ & 120.0 \\
\hline $\mathrm{Cl}(1)-\mathrm{Fe}(1)-\mathrm{Cl}(2)$ & $122.12(5)$ & $\mathrm{C}(5)-\mathrm{C}(6)-\mathrm{H}(6)$ & 120.0 \\
\hline $\mathrm{Cl}(1)-\mathrm{Fe}(1)-\mathrm{P}(1)$ & $108.13(4)$ & $C(6)-C(7)-C(8)$ & $120.7(4)$ \\
\hline $\mathrm{Cl}(2)-\mathrm{Fe}(1)-\mathrm{P}(1)$ & $106.62(4)$ & $\mathrm{C}(6)-\mathrm{C}(7)-\mathrm{H}(7)$ & 119.6 \\
\hline $\mathrm{Cl}(1)-\mathrm{Fe}(1)-\mathrm{P}(2)$ & $107.50(5)$ & $\mathrm{C}(8)-\mathrm{C}(7)-\mathrm{H}(7)$ & 119.6 \\
\hline $\mathrm{Cl}(2)-\mathrm{Fe}(1)-\mathrm{P}(2)$ & $102.66(4)$ & $C(7)-C(8)-C(15)$ & $117.0(3)$ \\
\hline $\mathrm{P}(1)-\mathrm{Fe}(1)-\mathrm{P}(2)$ & $109.30(4)$ & $\mathrm{C}(7)-\mathrm{C}(8)-\mathrm{P}(2)$ & $124.6(3)$ \\
\hline $\mathrm{C}(1)-\mathrm{P}(1)-\mathrm{C}(22)$ & 105.96(19) & $\mathrm{C}(15)-\mathrm{C}(8)-\mathrm{P}(2)$ & 118.1(3) \\
\hline$C(1)-P(1)-C(16)$ & $104.26(18)$ & $C(13)-C(9)-C(10)$ & $109.2(3)$ \\
\hline
\end{tabular}




\begin{tabular}{|c|c|c|c|}
\hline $\mathrm{C}(13)-\mathrm{C}(9)-\mathrm{C}(11)$ & $109.4(3)$ & $\mathrm{C}(19)-\mathrm{C}(18)-\mathrm{H}(18)$ & 120.0 \\
\hline$C(10)-C(9)-C(11)$ & 109.7(3) & $\mathrm{C}(17)-\mathrm{C}(18)-\mathrm{H}(18)$ & 120.0 \\
\hline$C(13)-C(9)-C(14)$ & $109.2(3)$ & $C(18)-C(19)-C(20)$ & $120.0(4)$ \\
\hline$C(10)-C(9)-C(14)$ & $109.4(3)$ & $\mathrm{C}(18)-\mathrm{C}(19)-\mathrm{H}(19)$ & 120.0 \\
\hline $\mathrm{C}(11)-\mathrm{C}(9)-\mathrm{C}(14)$ & 109.9(3) & $\mathrm{C}(20)-\mathrm{C}(19)-\mathrm{H}(19)$ & 120.0 \\
\hline $\mathrm{C}(9)-\mathrm{C}(10)-\mathrm{H}(10 \mathrm{~A})$ & 109.5 & $\mathrm{C}(21)-\mathrm{C}(20)-\mathrm{C}(19)$ & $120.2(4)$ \\
\hline $\mathrm{C}(9)-\mathrm{C}(10)-\mathrm{H}(10 \mathrm{~B})$ & 109.5 & $\mathrm{C}(21)-\mathrm{C}(20)-\mathrm{H}(20)$ & 119.9 \\
\hline $\mathrm{H}(10 \mathrm{~A})-\mathrm{C}(10)-\mathrm{H}(10 \mathrm{~B})$ & 109.5 & $\mathrm{C}(19)-\mathrm{C}(20)-\mathrm{H}(20)$ & 119.9 \\
\hline $\mathrm{C}(9)-\mathrm{C}(10)-\mathrm{H}(10 \mathrm{C})$ & 109.5 & $C(20)-C(21)-C(16)$ & $120.1(4)$ \\
\hline $\mathrm{H}(10 \mathrm{~A})-\mathrm{C}(10)-\mathrm{H}(10 \mathrm{C})$ & 109.5 & $\mathrm{C}(20)-\mathrm{C}(21)-\mathrm{H}(21)$ & 120.0 \\
\hline $\mathrm{H}(10 \mathrm{~B})-\mathrm{C}(10)-\mathrm{H}(10 \mathrm{C})$ & 109.5 & $\mathrm{C}(16)-\mathrm{C}(21)-\mathrm{H}(21)$ & 120.0 \\
\hline $\mathrm{C}(9)-\mathrm{C}(11)-\mathrm{H}(11 \mathrm{~A})$ & 109.5 & $\mathrm{C}(27)-\mathrm{C}(22)-\mathrm{C}(23)$ & $118.9(4)$ \\
\hline $\mathrm{C}(9)-\mathrm{C}(11)-\mathrm{H}(11 \mathrm{~B})$ & 109.5 & $\mathrm{C}(27)-\mathrm{C}(22)-\mathrm{P}(1)$ & $118.0(3)$ \\
\hline $\mathrm{H}(11 \mathrm{~A})-\mathrm{C}(11)-\mathrm{H}(11 \mathrm{~B})$ & 109.5 & $\mathrm{C}(23)-\mathrm{C}(22)-\mathrm{P}(1)$ & $123.1(3)$ \\
\hline $\mathrm{C}(9)-\mathrm{C}(11)-\mathrm{H}(11 \mathrm{C})$ & 109.5 & $C(22)-C(23)-C(24)$ & $120.6(4)$ \\
\hline $\mathrm{H}(11 \mathrm{~A})-\mathrm{C}(11)-\mathrm{H}(11 \mathrm{C})$ & 109.5 & $\mathrm{C}(22)-\mathrm{C}(23)-\mathrm{H}(23)$ & 119.7 \\
\hline $\mathrm{H}(11 \mathrm{~B})-\mathrm{C}(11)-\mathrm{H}(11 \mathrm{C})$ & 109.5 & $\mathrm{C}(24)-\mathrm{C}(23)-\mathrm{H}(23)$ & 119.7 \\
\hline $\mathrm{C}(13)-\mathrm{C}(12)-\mathrm{O}(1)$ & $122.2(3)$ & $C(25)-C(24)-C(23)$ & $119.2(4)$ \\
\hline$C(13)-C(12)-C(1)$ & $124.4(4)$ & $\mathrm{C}(25)-\mathrm{C}(24)-\mathrm{H}(24)$ & 120.4 \\
\hline $\mathrm{O}(1)-\mathrm{C}(12)-\mathrm{C}(1)$ & $113.4(3)$ & $\mathrm{C}(23)-\mathrm{C}(24)-\mathrm{H}(24)$ & 120.4 \\
\hline$C(12)-C(13)-C(4)$ & $116.0(4)$ & $C(26)-C(25)-C(24)$ & $120.2(4)$ \\
\hline$C(12)-C(13)-C(9)$ & $122.0(3)$ & $\mathrm{C}(26)-\mathrm{C}(25)-\mathrm{H}(25)$ & 119.9 \\
\hline $\mathrm{C}(4)-\mathrm{C}(13)-\mathrm{C}(9)$ & $121.9(4)$ & $\mathrm{C}(24)-\mathrm{C}(25)-\mathrm{H}(25)$ & 119.9 \\
\hline$C(15)-C(14)-C(5)$ & $116.4(4)$ & $C(25)-C(26)-C(27)$ & $120.6(5)$ \\
\hline$C(15)-C(14)-C(9)$ & $121.9(3)$ & $\mathrm{C}(25)-\mathrm{C}(26)-\mathrm{H}(26)$ & 119.7 \\
\hline$C(5)-C(14)-C(9)$ & $121.7(3)$ & $\mathrm{C}(27)-\mathrm{C}(26)-\mathrm{H}(26)$ & 119.7 \\
\hline $\mathrm{O}(1)-\mathrm{C}(15)-\mathrm{C}(14)$ & $121.9(3)$ & $C(26)-C(27)-C(22)$ & $120.5(4)$ \\
\hline $\mathrm{O}(1)-\mathrm{C}(15)-\mathrm{C}(8)$ & $114.5(3)$ & $\mathrm{C}(26)-\mathrm{C}(27)-\mathrm{H}(27)$ & 119.8 \\
\hline $\mathrm{C}(14)-\mathrm{C}(15)-\mathrm{C}(8)$ & $123.7(3)$ & $\mathrm{C}(22)-\mathrm{C}(27)-\mathrm{H}(27)$ & 119.8 \\
\hline$C(17)-C(16)-C(21)$ & $119.3(4)$ & $C(29)-C(28)-C(33)$ & $118.8(4)$ \\
\hline C(17)-C(16)-P(1) & $123.6(3)$ & $\mathrm{C}(29)-\mathrm{C}(28)-\mathrm{P}(2)$ & $123.3(3)$ \\
\hline$C(21)-C(16)-P(1)$ & $117.2(3)$ & $\mathrm{C}(33)-\mathrm{C}(28)-\mathrm{P}(2)$ & $117.7(3)$ \\
\hline$C(16)-C(17)-C(18)$ & $120.3(4)$ & $\mathrm{C}(30)-\mathrm{C}(29)-\mathrm{C}(28)$ & $120.0(4)$ \\
\hline $\mathrm{C}(16)-\mathrm{C}(17)-\mathrm{H}(17)$ & 119.8 & $\mathrm{C}(30)-\mathrm{C}(29)-\mathrm{H}(29)$ & 120.0 \\
\hline $\mathrm{C}(18)-\mathrm{C}(17)-\mathrm{H}(17)$ & 119.8 & $\mathrm{C}(28)-\mathrm{C}(29)-\mathrm{H}(29)$ & 120.0 \\
\hline C(19)-C(18)-C(17) & $120.1(4)$ & $\mathrm{C}(31)-\mathrm{C}(30)-\mathrm{C}(29)$ & $120.8(4)$ \\
\hline
\end{tabular}




\begin{tabular}{|c|c|c|c|}
\hline $\mathrm{C}(31)-\mathrm{C}(30)-\mathrm{H}(30)$ & 119.6 & $\mathrm{C}(61)-\mathrm{P}(3)-\mathrm{C}(40)$ & $106.63(18)$ \\
\hline $\mathrm{C}(29)-\mathrm{C}(30)-\mathrm{H}(30)$ & 119.6 & $\mathrm{C}(55)-\mathrm{P}(3)-\mathrm{C}(40)$ & $104.58(18)$ \\
\hline $\mathrm{C}(30)-\mathrm{C}(31)-\mathrm{C}(32)$ & $119.7(4)$ & $\mathrm{C}(61)-\mathrm{P}(3)-\mathrm{Fe}(2)$ & $119.50(13)$ \\
\hline $\mathrm{C}(30)-\mathrm{C}(31)-\mathrm{H}(31)$ & 120.2 & $\mathrm{C}(55)-\mathrm{P}(3)-\mathrm{Fe}(2)$ & $111.40(14)$ \\
\hline $\mathrm{C}(32)-\mathrm{C}(31)-\mathrm{H}(31)$ & 120.2 & $\mathrm{C}(40)-\mathrm{P}(3)-\mathrm{Fe}(2)$ & $110.86(13)$ \\
\hline $\mathrm{C}(33)-\mathrm{C}(32)-\mathrm{C}(31)$ & $119.7(4)$ & $\mathrm{C}(73)-\mathrm{P}(4)-\mathrm{C}(67)$ & $104.47(18)$ \\
\hline $\mathrm{C}(33)-\mathrm{C}(32)-\mathrm{H}(32)$ & 120.1 & $\mathrm{C}(73)-\mathrm{P}(4)-\mathrm{C}(47)$ & $103.33(18)$ \\
\hline $\mathrm{C}(31)-\mathrm{C}(32)-\mathrm{H}(32)$ & 120.1 & $\mathrm{C}(67)-\mathrm{P}(4)-\mathrm{C}(47)$ & $105.86(18)$ \\
\hline $\mathrm{C}(32)-\mathrm{C}(33)-\mathrm{C}(28)$ & $121.0(4)$ & $\mathrm{C}(73)-\mathrm{P}(4)-\mathrm{Fe}(2)$ & $112.72(14)$ \\
\hline C(32)-C(33)-H(33) & 119.5 & $\mathrm{C}(67)-\mathrm{P}(4)-\mathrm{Fe}(2)$ & $119.85(13)$ \\
\hline $\mathrm{C}(28)-\mathrm{C}(33)-\mathrm{H}(33)$ & 119.5 & $\mathrm{C}(47)-\mathrm{P}(4)-\mathrm{Fe}(2)$ & $109.19(13)$ \\
\hline C(39)-C(34)-C(35) & $118.8(4)$ & $\mathrm{C}(54)-\mathrm{O}(2)-\mathrm{C}(51)$ & $117.7(3)$ \\
\hline C(39)-C(34)-P(2) & $118.2(3)$ & $\mathrm{C}(41)-\mathrm{C}(40)-\mathrm{C}(51)$ & $117.9(3)$ \\
\hline $\mathrm{C}(35)-\mathrm{C}(34)-\mathrm{P}(2)$ & $122.8(3)$ & $\mathrm{C}(41)-\mathrm{C}(40)-\mathrm{P}(3)$ & $123.6(3)$ \\
\hline$C(36)-C(35)-C(34)$ & $120.2(4)$ & $\mathrm{C}(51)-\mathrm{C}(40)-\mathrm{P}(3)$ & $118.3(3)$ \\
\hline $\mathrm{C}(36)-\mathrm{C}(35)-\mathrm{H}(35)$ & 119.9 & $C(42)-C(41)-C(40)$ & $119.4(4)$ \\
\hline $\mathrm{C}(34)-\mathrm{C}(35)-\mathrm{H}(35)$ & 119.9 & $\mathrm{C}(42)-\mathrm{C}(41)-\mathrm{H}(41)$ & 120.3 \\
\hline $\mathrm{C}(37)-\mathrm{C}(36)-\mathrm{C}(35)$ & $120.3(4)$ & $\mathrm{C}(40)-\mathrm{C}(41)-\mathrm{H}(41)$ & 120.3 \\
\hline $\mathrm{C}(37)-\mathrm{C}(36)-\mathrm{H}(36)$ & 119.8 & $C(43)-C(42)-C(41)$ & $120.9(4)$ \\
\hline $\mathrm{C}(35)-\mathrm{C}(36)-\mathrm{H}(36)$ & 119.8 & $\mathrm{C}(43)-\mathrm{C}(42)-\mathrm{H}(42)$ & 119.5 \\
\hline $\mathrm{C}(36)-\mathrm{C}(37)-\mathrm{C}(38)$ & $120.6(4)$ & $\mathrm{C}(41)-\mathrm{C}(42)-\mathrm{H}(42)$ & 119.5 \\
\hline $\mathrm{C}(36)-\mathrm{C}(37)-\mathrm{H}(37)$ & 119.7 & $\mathrm{C}(42)-\mathrm{C}(43)-\mathrm{C}(52)$ & $121.8(4)$ \\
\hline $\mathrm{C}(38)-\mathrm{C}(37)-\mathrm{H}(37)$ & 119.7 & $\mathrm{C}(42)-\mathrm{C}(43)-\mathrm{H}(43)$ & 119.1 \\
\hline $\mathrm{C}(37)-\mathrm{C}(38)-\mathrm{C}(39)$ & $119.2(4)$ & $\mathrm{C}(52)-\mathrm{C}(43)-\mathrm{H}(43)$ & 119.1 \\
\hline $\mathrm{C}(37)-\mathrm{C}(38)-\mathrm{H}(38)$ & 120.4 & $C(45)-C(44)-C(53)$ & $121.5(4)$ \\
\hline C(39)-C(38)-H(38) & 120.4 & $\mathrm{C}(45)-\mathrm{C}(44)-\mathrm{H}(44)$ & 119.3 \\
\hline C(34)-C(39)-C(38) & $120.9(4)$ & $\mathrm{C}(53)-\mathrm{C}(44)-\mathrm{H}(44)$ & 119.3 \\
\hline C(34)-C(39)-H(39) & 119.6 & $C(44)-C(45)-C(46)$ & $120.5(4)$ \\
\hline C(38)-C(39)-H(39) & 119.6 & $\mathrm{C}(44)-\mathrm{C}(45)-\mathrm{H}(45)$ & 119.8 \\
\hline $\mathrm{Cl}(3)-\mathrm{Fe}(2)-\mathrm{Cl}(4)$ & $124.40(5)$ & $\mathrm{C}(46)-\mathrm{C}(45)-\mathrm{H}(45)$ & 119.8 \\
\hline $\mathrm{Cl}(3)-\mathrm{Fe}(2)-\mathrm{P}(3)$ & $108.03(5)$ & $\mathrm{C}(47)-\mathrm{C}(46)-\mathrm{C}(45)$ & $119.6(4)$ \\
\hline $\mathrm{Cl}(4)-\mathrm{Fe}(2)-\mathrm{P}(3)$ & $101.29(4)$ & $\mathrm{C}(47)-\mathrm{C}(46)-\mathrm{H}(46)$ & 120.2 \\
\hline $\mathrm{Cl}(3)-\mathrm{Fe}(2)-\mathrm{P}(4)$ & $107.09(4)$ & $\mathrm{C}(45)-\mathrm{C}(46)-\mathrm{H}(46)$ & 120.2 \\
\hline $\mathrm{Cl}(4)-\mathrm{Fe}(2)-\mathrm{P}(4)$ & $106.34(4)$ & $\mathrm{C}(46)-\mathrm{C}(47)-\mathrm{C}(54)$ & $118.6(4)$ \\
\hline$P(3)-F e(2)-P(4)$ & $109.00(4)$ & $\mathrm{C}(46)-\mathrm{C}(47)-\mathrm{P}(4)$ & $124.3(3)$ \\
\hline $\mathrm{C}(61)-\mathrm{P}(3)-\mathrm{C}(55)$ & $102.60(19)$ & $\mathrm{C}(54)-\mathrm{C}(47)-\mathrm{P}(4)$ & $117.0(3)$ \\
\hline
\end{tabular}




\begin{tabular}{|c|c|}
\hline$C(52)-C(48)-C(53)$ & $109.2(3)$ \\
\hline$C(52)-C(48)-C(49)$ & $109.4(3)$ \\
\hline$C(53)-C(48)-C(49)$ & 109.1(3) \\
\hline $\mathrm{C}(52)-\mathrm{C}(48)-\mathrm{C}(50)$ & $110.5(3)$ \\
\hline$C(53)-C(48)-C(50)$ & 109.1(3) \\
\hline $\mathrm{C}(49)-\mathrm{C}(48)-\mathrm{C}(50)$ & $109.6(3)$ \\
\hline $\mathrm{C}(48)-\mathrm{C}(49)-\mathrm{H}(49 \mathrm{~A})$ & 109.5 \\
\hline $\mathrm{C}(48)-\mathrm{C}(49)-\mathrm{H}(49 \mathrm{~B})$ & 109.5 \\
\hline H(49A)-C(49)-H(49B) & 109.5 \\
\hline $\mathrm{C}(48)-\mathrm{C}(49)-\mathrm{H}(49 \mathrm{C})$ & 109.5 \\
\hline $\mathrm{H}(49 \mathrm{~A})-\mathrm{C}(49)-\mathrm{H}(49 \mathrm{C})$ & 109.5 \\
\hline H(49B)-C(49)-H(49C) & 109.5 \\
\hline $\mathrm{C}(48)-\mathrm{C}(50)-\mathrm{H}(50 \mathrm{~A})$ & 109.5 \\
\hline $\mathrm{C}(48)-\mathrm{C}(50)-\mathrm{H}(50 \mathrm{~B})$ & 109.5 \\
\hline $\mathrm{H}(50 \mathrm{~A})-\mathrm{C}(50)-\mathrm{H}(50 \mathrm{~B})$ & 109.5 \\
\hline $\mathrm{C}(48)-\mathrm{C}(50)-\mathrm{H}(50 \mathrm{C})$ & 109.5 \\
\hline $\mathrm{H}(50 \mathrm{~A})-\mathrm{C}(50)-\mathrm{H}(50 \mathrm{C})$ & 109.5 \\
\hline $\mathrm{H}(50 \mathrm{~B})-\mathrm{C}(50)-\mathrm{H}(50 \mathrm{C})$ & 109.5 \\
\hline $\mathrm{C}(52)-\mathrm{C}(51)-\mathrm{O}(2)$ & $121.6(3)$ \\
\hline$C(52)-C(51)-C(40)$ & $124.2(3)$ \\
\hline $\mathrm{O}(2)-\mathrm{C}(51)-\mathrm{C}(40)$ & $114.2(3)$ \\
\hline$C(51)-C(52)-C(43)$ & $115.7(4)$ \\
\hline $\mathrm{C}(51)-\mathrm{C}(52)-\mathrm{C}(48)$ & $122.6(3)$ \\
\hline $\mathrm{C}(43)-\mathrm{C}(52)-\mathrm{C}(48)$ & $121.7(4)$ \\
\hline$C(54)-C(53)-C(44)$ & $116.1(3)$ \\
\hline$C(54)-C(53)-C(48)$ & $122.0(3)$ \\
\hline $\mathrm{C}(44)-\mathrm{C}(53)-\mathrm{C}(48)$ & $121.9(3)$ \\
\hline $\mathrm{C}(53)-\mathrm{C}(54)-\mathrm{O}(2)$ & $122.3(3)$ \\
\hline$C(53)-C(54)-C(47)$ & $123.7(4)$ \\
\hline $\mathrm{O}(2)-\mathrm{C}(54)-\mathrm{C}(47)$ & $114.0(3)$ \\
\hline$C(60)-C(55)-C(56)$ & $119.1(4)$ \\
\hline $\mathrm{C}(60)-\mathrm{C}(55)-\mathrm{P}(3)$ & $118.8(3)$ \\
\hline $\mathrm{C}(56)-\mathrm{C}(55)-\mathrm{P}(3)$ & $122.1(3)$ \\
\hline $\mathrm{C}(57)-\mathrm{C}(56)-\mathrm{C}(55)$ & $119.6(4)$ \\
\hline $\mathrm{C}(57)-\mathrm{C}(56)-\mathrm{H}(56)$ & 120.2 \\
\hline $\mathrm{C}(55)-\mathrm{C}(56)-\mathrm{H}(56)$ & 120.2 \\
\hline
\end{tabular}

\begin{tabular}{|c|c|}
\hline $\mathrm{C}(58)-\mathrm{C}(57)-\mathrm{C}(56)$ & $120.4(4)$ \\
\hline $\mathrm{C}(58)-\mathrm{C}(57)-\mathrm{H}(57)$ & 119.8 \\
\hline $\mathrm{C}(56)-\mathrm{C}(57)-\mathrm{H}(57)$ & 119.8 \\
\hline $\mathrm{C}(57)-\mathrm{C}(58)-\mathrm{C}(59)$ & $120.7(4)$ \\
\hline $\mathrm{C}(57)-\mathrm{C}(58)-\mathrm{H}(58)$ & 119.6 \\
\hline C(59)-C(58)-H(58) & 119.6 \\
\hline $\mathrm{C}(58)-\mathrm{C}(59)-\mathrm{C}(60)$ & 119.1(4) \\
\hline C(58)-C(59)-H(59) & 120.5 \\
\hline $\mathrm{C}(60)-\mathrm{C}(59)-\mathrm{H}(59)$ & 120.5 \\
\hline $\mathrm{C}(55)-\mathrm{C}(60)-\mathrm{C}(59)$ & 121.1(4) \\
\hline $\mathrm{C}(55)-\mathrm{C}(60)-\mathrm{H}(60)$ & 119.5 \\
\hline $\mathrm{C}(59)-\mathrm{C}(60)-\mathrm{H}(60)$ & 119.5 \\
\hline $\mathrm{C}(62)-\mathrm{C}(61)-\mathrm{C}(66)$ & $118.7(4)$ \\
\hline $\mathrm{C}(62)-\mathrm{C}(61)-\mathrm{P}(3)$ & $123.0(3)$ \\
\hline C(66)-C(61)-P(3) & $118.2(3)$ \\
\hline $\mathrm{C}(63)-\mathrm{C}(62)-\mathrm{C}(61)$ & $120.8(4)$ \\
\hline $\mathrm{C}(63)-\mathrm{C}(62)-\mathrm{H}(62)$ & 119.6 \\
\hline $\mathrm{C}(61)-\mathrm{C}(62)-\mathrm{H}(62)$ & 119.6 \\
\hline$C(64)-C(63)-C(62)$ & $119.3(4)$ \\
\hline $\mathrm{C}(64)-\mathrm{C}(63)-\mathrm{H}(63)$ & 120.4 \\
\hline $\mathrm{C}(62)-\mathrm{C}(63)-\mathrm{H}(63)$ & 120.4 \\
\hline $\mathrm{C}(63)-\mathrm{C}(64)-\mathrm{C}(65)$ & 121.1(4) \\
\hline $\mathrm{C}(63)-\mathrm{C}(64)-\mathrm{H}(64)$ & 119.4 \\
\hline C(65)-C(64)-H(64) & 119.4 \\
\hline$C(64)-C(65)-C(66)$ & 119.3(4) \\
\hline $\mathrm{C}(64)-\mathrm{C}(65)-\mathrm{H}(65)$ & 120.3 \\
\hline $\mathrm{C}(66)-\mathrm{C}(65)-\mathrm{H}(65)$ & 120.3 \\
\hline $\mathrm{C}(65)-\mathrm{C}(66)-\mathrm{C}(61)$ & $120.8(4)$ \\
\hline $\mathrm{C}(65)-\mathrm{C}(66)-\mathrm{H}(66)$ & 119.6 \\
\hline $\mathrm{C}(61)-\mathrm{C}(66)-\mathrm{H}(66)$ & 119.6 \\
\hline $\mathrm{C}(68)-\mathrm{C}(67)-\mathrm{C}(72)$ & 119.2(4) \\
\hline $\mathrm{C}(68)-\mathrm{C}(67)-\mathrm{P}(4)$ & $123.6(3)$ \\
\hline$C(72)-C(67)-P(4)$ & 117.2(3) \\
\hline $\mathrm{C}(69)-\mathrm{C}(68)-\mathrm{C}(67)$ & $120.2(4)$ \\
\hline C(69)-C(68)-H(68) & 119.9 \\
\hline $\mathrm{C}(67)-\mathrm{C}(68)-\mathrm{H}(68)$ & 119.9 \\
\hline
\end{tabular}




$\begin{array}{llll}\mathrm{C}(70)-\mathrm{C}(69)-\mathrm{C}(68) & 120.5(4) & \mathrm{C}(75)-\mathrm{C}(74)-\mathrm{C}(73) & 119.3(4) \\ \mathrm{C}(70)-\mathrm{C}(69)-\mathrm{H}(69) & 119.7 & \mathrm{C}(75)-\mathrm{C}(74)-\mathrm{H}(74) & 120.4 \\ \mathrm{C}(68)-\mathrm{C}(69)-\mathrm{H}(69) & 119.7 & \mathrm{C}(73)-\mathrm{C}(74)-\mathrm{H}(74) & 120.4 \\ \mathrm{C}(69)-\mathrm{C}(70)-\mathrm{C}(71) & 120.2(4) & \mathrm{C}(74)-\mathrm{C}(75)-\mathrm{C}(76) & 120.6(4) \\ \mathrm{C}(69)-\mathrm{C}(70)-\mathrm{H}(70) & 119.9 & \mathrm{C}(74)-\mathrm{C}(75)-\mathrm{H}(75) & 119.7 \\ \mathrm{C}(71)-\mathrm{C}(70)-\mathrm{H}(70) & 119.9 & \mathrm{C}(76)-\mathrm{C}(75)-\mathrm{H}(75) & 119.7 \\ \mathrm{C}(70)-\mathrm{C}(71)-\mathrm{C}(72) & 120.1(4) & \mathrm{C}(77)-\mathrm{C}(76)-\mathrm{C}(75) & 120.7(4) \\ \mathrm{C}(70)-\mathrm{C}(71)-\mathrm{H}(71) & 120.0 & \mathrm{C}(77)-\mathrm{C}(76)-\mathrm{H}(76) & 119.7 \\ \mathrm{C}(72)-\mathrm{C}(71)-\mathrm{H}(71) & 120.0 & \mathrm{C}(75)-\mathrm{C}(76)-\mathrm{H}(76) & 119.7 \\ \mathrm{C}(71)-\mathrm{C}(72)-\mathrm{C}(67) & 119.8(4) & \mathrm{C}(76)-\mathrm{C}(77)-\mathrm{C}(78) & 119.4(4) \\ \mathrm{C}(71)-\mathrm{C}(72)-\mathrm{H}(72) & 120.1 & \mathrm{C}(76)-\mathrm{C}(77)-\mathrm{H}(77) & 120.3 \\ \mathrm{C}(67)-\mathrm{C}(72)-\mathrm{H}(72) & 120.1 & \mathrm{C}(78)-\mathrm{C}(77)-\mathrm{H}(77) & 120.3 \\ \mathrm{C}(78)-\mathrm{C}(73)-\mathrm{C}(74) & 119.2(4) & \mathrm{C}(73)-\mathrm{C}(78)-\mathrm{C}(77) & 120.8(4) \\ \mathrm{C}(78)-\mathrm{C}(73)-\mathrm{P}(4) & 117.4(3) & \mathrm{C}(73)-\mathrm{C}(78)-\mathrm{H}(78) & 119.6 \\ \mathrm{C}(74)-\mathrm{C}(73)-\mathrm{P}(4) & 123.3(3) & \mathrm{C}(77)-\mathrm{C}(78)-\mathrm{H}(78) & 119.6\end{array}$


Table S22. Anisotropic displacement parameters $\left(\AA^{2} \times 10^{3}\right)$ for neivf02. The anisotropic displacement factor exponent takes the form: $-2 \pi^{2}\left[h^{2} a^{* 2} U_{11}+\ldots+2 h k a^{*} b^{*} U_{12}\right]$

\begin{tabular}{|c|c|c|c|c|c|c|}
\hline & $\mathrm{U}_{11}$ & $\mathrm{U}_{22}$ & $\mathrm{U}_{33}$ & $\mathrm{U}_{23}$ & $\mathrm{U}_{13}$ & $\mathrm{U}_{12}$ \\
\hline $\mathrm{Fe} 1$ & 11(1) & $16(1)$ & $17(1)$ & $0(1)$ & $1(1)$ & $0(1)$ \\
\hline $\mathrm{Cl1}$ & $22(1)$ & $35(1)$ & $26(1)$ & $-8(1)$ & $7(1)$ & $3(1)$ \\
\hline $\mathrm{Cl} 2$ & $18(1)$ & $20(1)$ & $21(1)$ & $5(1)$ & $2(1)$ & $1(1)$ \\
\hline P1 & 11(1) & $16(1)$ & $18(1)$ & $0(1)$ & $3(1)$ & $0(1)$ \\
\hline P2 & $12(1)$ & $13(1)$ & $17(1)$ & $0(1)$ & $1(1)$ & $1(1)$ \\
\hline O1 & $9(1)$ & $20(2)$ & $16(1)$ & $-1(1)$ & $0(1)$ & $-5(1)$ \\
\hline $\mathrm{C} 1$ & $15(2)$ & $17(2)$ & $15(2)$ & $-3(2)$ & $4(2)$ & $-2(2)$ \\
\hline $\mathrm{C} 2$ & $14(2)$ & $27(2)$ & $24(2)$ & $-2(2)$ & $3(2)$ & $-5(2)$ \\
\hline $\mathrm{C} 3$ & $24(2)$ & $34(3)$ & $19(2)$ & $-8(2)$ & $-1(2)$ & $-11(2)$ \\
\hline $\mathrm{C} 4$ & $27(2)$ & $27(2)$ & $19(2)$ & $-4(2)$ & $4(2)$ & $-3(2)$ \\
\hline $\mathrm{C} 5$ & $14(2)$ & $29(2)$ & $21(2)$ & $0(2)$ & $6(2)$ & $3(2)$ \\
\hline C6 & $9(2)$ & $25(2)$ & $29(2)$ & $-1(2)$ & $2(2)$ & $-5(2)$ \\
\hline $\mathrm{C} 7$ & $12(2)$ & $19(2)$ & $23(2)$ & $2(2)$ & $-3(2)$ & $1(2)$ \\
\hline $\mathrm{C} 8$ & $12(2)$ & $15(2)$ & $17(2)$ & $2(2)$ & $1(2)$ & $6(2)$ \\
\hline C9 & $18(2)$ & $20(2)$ & $17(2)$ & $-2(2)$ & $3(2)$ & $2(2)$ \\
\hline $\mathrm{C} 10$ & $26(2)$ & $23(2)$ & $22(2)$ & $-4(2)$ & $4(2)$ & $7(2)$ \\
\hline C11 & $18(2)$ & $30(3)$ & $22(2)$ & $-1(2)$ & $2(2)$ & $-2(2)$ \\
\hline $\mathrm{C} 12$ & $12(2)$ & $16(2)$ & $20(2)$ & $-1(2)$ & $-2(2)$ & $1(2)$ \\
\hline $\mathrm{C} 13$ & $15(2)$ & $15(2)$ & $20(2)$ & $-2(2)$ & $1(2)$ & $-3(2)$ \\
\hline $\mathrm{C} 14$ & $11(2)$ & $19(2)$ & $18(2)$ & $-1(2)$ & $3(2)$ & $4(2)$ \\
\hline $\mathrm{C} 15$ & $5(2)$ & $15(2)$ & $22(2)$ & $1(2)$ & $0(2)$ & $4(2)$ \\
\hline $\mathrm{C} 16$ & $12(2)$ & $19(2)$ & $17(2)$ & $-1(2)$ & $4(2)$ & $-1(2)$ \\
\hline $\mathrm{C} 17$ & $18(2)$ & $17(2)$ & $23(2)$ & $0(2)$ & $2(2)$ & $-2(2)$ \\
\hline $\mathrm{C} 18$ & $13(2)$ & $19(2)$ & $28(2)$ & $-1(2)$ & $3(2)$ & $-8(2)$ \\
\hline C19 & $12(2)$ & $34(3)$ & $21(2)$ & $-2(2)$ & $-2(2)$ & $6(2)$ \\
\hline $\mathrm{C} 20$ & $17(2)$ & $25(2)$ & $23(2)$ & $1(2)$ & $0(2)$ & $4(2)$ \\
\hline $\mathrm{C} 21$ & $19(2)$ & $23(2)$ & $20(2)$ & $1(2)$ & $2(2)$ & $-1(2)$ \\
\hline C22 & $6(2)$ & $29(2)$ & $23(2)$ & $-3(2)$ & $-1(2)$ & $-1(2)$ \\
\hline $\mathrm{C} 23$ & $17(2)$ & $25(2)$ & $21(2)$ & $-3(2)$ & $0(2)$ & $-1(2)$ \\
\hline $\mathrm{C} 24$ & $22(2)$ & $11(2)$ & $37(3)$ & $-2(2)$ & $5(2)$ & $2(2)$ \\
\hline $\mathrm{C} 25$ & $24(2)$ & $18(2)$ & $41(3)$ & $8(2)$ & $1(2)$ & $1(2)$ \\
\hline
\end{tabular}




\begin{tabular}{|c|c|c|c|c|c|c|}
\hline $\mathrm{C} 26$ & $41(3)$ & $29(3)$ & $27(3)$ & $4(2)$ & $4(2)$ & $2(2)$ \\
\hline $\mathrm{C} 27$ & $32(3)$ & $23(2)$ & 27(3) & $-3(2)$ & $3(2)$ & $4(2)$ \\
\hline C28 & $14(2)$ & $13(2)$ & $20(2)$ & $-2(2)$ & $-4(2)$ & $-1(2)$ \\
\hline C29 & $27(2)$ & $20(2)$ & $22(2)$ & $1(2)$ & $3(2)$ & $3(2)$ \\
\hline C30 & $33(3)$ & $22(2)$ & 29(3) & $2(2)$ & $1(2)$ & $7(2)$ \\
\hline C31 & $42(3)$ & $20(2)$ & $21(2)$ & $4(2)$ & $-4(2)$ & $0(2)$ \\
\hline C32 & $29(2)$ & $27(3)$ & $25(2)$ & $2(2)$ & $6(2)$ & $0(2)$ \\
\hline C33 & $20(2)$ & $22(2)$ & $27(2)$ & $3(2)$ & $3(2)$ & $6(2)$ \\
\hline C34 & $17(2)$ & $13(2)$ & $17(2)$ & $0(2)$ & $4(2)$ & $-2(2)$ \\
\hline C35 & $19(2)$ & $16(2)$ & $24(2)$ & $1(2)$ & $2(2)$ & $1(2)$ \\
\hline C36 & $18(2)$ & $27(2)$ & $27(2)$ & $-2(2)$ & $-7(2)$ & $-1(2)$ \\
\hline C37 & $22(2)$ & $20(2)$ & $31(3)$ & $-8(2)$ & $-2(2)$ & $-3(2)$ \\
\hline C38 & $27(2)$ & $19(2)$ & $38(3)$ & $-5(2)$ & $-4(2)$ & $3(2)$ \\
\hline C39 & $18(2)$ & $18(2)$ & $24(2)$ & $0(2)$ & $-3(2)$ & $-1(2)$ \\
\hline $\mathrm{Fe} 2$ & $10(1)$ & $15(1)$ & $17(1)$ & $1(1)$ & $2(1)$ & $1(1)$ \\
\hline $\mathrm{Cl} 3$ & $23(1)$ & $39(1)$ & $26(1)$ & 11(1) & $8(1)$ & $-2(1)$ \\
\hline $\mathrm{Cl} 4$ & $17(1)$ & $18(1)$ & 21(1) & $-4(1)$ & $1(1)$ & 1(1) \\
\hline P3 & $10(1)$ & $19(1)$ & $16(1)$ & $1(1)$ & $1(1)$ & 1(1) \\
\hline P4 & $10(1)$ & $15(1)$ & $18(1)$ & $0(1)$ & $2(1)$ & $1(1)$ \\
\hline $\mathrm{O} 2$ & $12(1)$ & $18(2)$ & $14(1)$ & $-1(1)$ & $2(1)$ & 2(1) \\
\hline $\mathrm{C} 40$ & $11(2)$ & $16(2)$ & $19(2)$ & $-2(2)$ & $4(2)$ & $1(2)$ \\
\hline C41 & $15(2)$ & $18(2)$ & $21(2)$ & $3(2)$ & $-2(2)$ & $4(2)$ \\
\hline $\mathrm{C} 42$ & $10(2)$ & $28(2)$ & $27(2)$ & $0(2)$ & $4(2)$ & $2(2)$ \\
\hline C43 & $15(2)$ & $26(2)$ & $20(2)$ & $-3(2)$ & $6(2)$ & $-2(2)$ \\
\hline $\mathrm{C} 44$ & $22(2)$ & $34(3)$ & $17(2)$ & $3(2)$ & $5(2)$ & $9(2)$ \\
\hline $\mathrm{C} 45$ & $19(2)$ & $40(3)$ & $20(2)$ & $3(2)$ & $-2(2)$ & $8(2)$ \\
\hline C46 & $11(2)$ & $24(2)$ & $23(2)$ & $4(2)$ & $2(2)$ & $7(2)$ \\
\hline $\mathrm{C} 47$ & $15(2)$ & $14(2)$ & $17(2)$ & $2(2)$ & $3(2)$ & $-1(2)$ \\
\hline C48 & $11(2)$ & $22(2)$ & $15(2)$ & $-1(2)$ & $3(2)$ & $-2(2)$ \\
\hline C49 & $23(2)$ & $30(3)$ & $18(2)$ & $6(2)$ & $2(2)$ & $-4(2)$ \\
\hline C50 & $13(2)$ & $30(3)$ & $26(2)$ & $-3(2)$ & $1(2)$ & $0(2)$ \\
\hline C51 & $6(2)$ & $16(2)$ & $20(2)$ & $-3(2)$ & $1(2)$ & $1(2)$ \\
\hline C52 & $13(2)$ & $15(2)$ & $23(2)$ & $1(2)$ & $1(2)$ & $-3(2)$ \\
\hline C53 & $12(2)$ & $17(2)$ & $18(2)$ & $1(2)$ & $1(2)$ & $-1(2)$ \\
\hline C54 & $13(2)$ & $15(2)$ & $15(2)$ & $1(2)$ & $-1(2)$ & $0(2)$ \\
\hline C55 & $11(2)$ & $33(3)$ & $14(2)$ & $3(2)$ & $2(2)$ & $5(2)$ \\
\hline
\end{tabular}




\begin{tabular}{lllllll} 
C56 & $19(2)$ & $30(3)$ & $20(2)$ & $4(2)$ & $-1(2)$ & $0(2)$ \\
C57 & $18(2)$ & $42(3)$ & $22(2)$ & $4(2)$ & $-3(2)$ & $6(2)$ \\
C58 & $23(2)$ & $26(3)$ & $30(3)$ & $7(2)$ & $3(2)$ & $12(2)$ \\
C59 & $30(2)$ & $19(2)$ & $32(3)$ & $3(2)$ & $1(2)$ & $5(2)$ \\
C60 & $16(2)$ & $23(2)$ & $22(2)$ & $-2(2)$ & $0(2)$ & $0(2)$ \\
C61 & $16(2)$ & $21(2)$ & $18(2)$ & $1(2)$ & $-1(2)$ & $1(2)$ \\
C62 & $17(2)$ & $22(2)$ & $24(2)$ & $3(2)$ & $1(2)$ & $0(2)$ \\
C63 & $24(2)$ & $21(2)$ & $24(2)$ & $-2(2)$ & $-3(2)$ & $-6(2)$ \\
C64 & $23(2)$ & $28(3)$ & $25(2)$ & $-6(2)$ & $-2(2)$ & $1(2)$ \\
C65 & $25(2)$ & $35(3)$ & $24(2)$ & $-8(2)$ & $5(2)$ & $-2(2)$ \\
C66 & $24(2)$ & $34(3)$ & $24(2)$ & $-8(2)$ & $5(2)$ & $-5(2)$ \\
C67 & $10(2)$ & $13(2)$ & $19(2)$ & $-3(2)$ & $1(2)$ & $2(2)$ \\
C68 & $21(2)$ & $17(2)$ & $24(2)$ & $2(2)$ & $3(2)$ & $-3(2)$ \\
C69 & $18(2)$ & $23(2)$ & $37(3)$ & $6(2)$ & $3(2)$ & $-1(2)$ \\
C70 & $27(2)$ & $26(3)$ & $25(2)$ & $-9(2)$ & $-3(2)$ & $-2(2)$ \\
C71 & $46(3)$ & $31(3)$ & $24(3)$ & $-6(2)$ & $6(2)$ & $2(2)$ \\
C72 & $38(3)$ & $15(2)$ & $25(2)$ & $-1(2)$ & $4(2)$ & $2(2)$ \\
C73 & $13(2)$ & $23(2)$ & $17(2)$ & $2(2)$ & $1(2)$ & $-3(2)$ \\
C74 & $14(2)$ & $20(2)$ & $27(2)$ & $-2(2)$ & $4(2)$ & $-1(2)$ \\
C75 & $15(2)$ & $28(3)$ & $28(3)$ & $-3(2)$ & $4(2)$ & $3(2)$ \\
C76 & $10(2)$ & $29(2)$ & $23(2)$ & $1(2)$ & $1(2)$ & $4(2)$ \\
C77 & $19(2)$ & $20(2)$ & $28(2)$ & $2(2)$ & $-3(2)$ & $-3(2)$ \\
C78 & $17(2)$ & $13(2)$ & $24(2)$ & $2(2)$ & $2(2)$ & $2(2)$ \\
& & & & & & \\
\hline
\end{tabular}


Table S23. Hydrogen coordinates $\left(\times 10^{4}\right)$ and isotropic displacement parameters $\left(\AA^{2} \times 10^{3}\right)$ for neivf02.

\begin{tabular}{|c|c|c|c|c|}
\hline & $\mathrm{x}$ & $\mathrm{y}$ & $\mathrm{z}$ & $\mathrm{U}(\mathrm{eq})$ \\
\hline $\mathrm{H} 2$ & 4752 & 5961 & 2127 & 26 \\
\hline H3 & 4263 & 6178 & 2688 & 31 \\
\hline H4 & 2920 & 5906 & 2767 & 29 \\
\hline H5 & 251 & 4221 & 2172 & 25 \\
\hline H6 & -348 & 3300 & 1664 & 25 \\
\hline $\mathrm{H} 7$ & 328 & 3181 & 1146 & 22 \\
\hline $\mathrm{H} 10 \mathrm{~A}$ & 1335 & 7148 & 2155 & 36 \\
\hline H10B & 1550 & 7020 & 2572 & 36 \\
\hline $\mathrm{H} 10 \mathrm{C}$ & 726 & 6475 & 2405 & 36 \\
\hline $\mathrm{H} 11 \mathrm{~A}$ & 1878 & 3660 & 2592 & 35 \\
\hline H11B & 1028 & 4274 & 2648 & 35 \\
\hline $\mathrm{H} 11 \mathrm{C}$ & 1809 & 4883 & 2840 & 35 \\
\hline H17 & 5375 & 7015 & 1389 & 23 \\
\hline H18 & 6751 & 6688 & 1439 & 24 \\
\hline H19 & 7258 & 4684 & 1576 & 27 \\
\hline $\mathrm{H} 20$ & 6397 & 2967 & 1633 & 26 \\
\hline H21 & 5027 & 3266 & 1565 & 24 \\
\hline $\mathrm{H} 23$ & 3726 & 7997 & 1596 & 25 \\
\hline $\mathrm{H} 24$ & 3614 & 9886 & 1265 & 28 \\
\hline $\mathrm{H} 25$ & 3595 & 9792 & 645 & 33 \\
\hline H26 & 3733 & 7859 & 363 & 39 \\
\hline $\mathrm{H} 27$ & 3881 & 5996 & 690 & 32 \\
\hline H29 & 842 & 5984 & 910 & 27 \\
\hline H30 & 612 & 7597 & 495 & 34 \\
\hline H31 & 1379 & 7757 & 5 & 34 \\
\hline H32 & 2409 & 6314 & -65 & 32 \\
\hline H33 & 2674 & 4747 & 358 & 28 \\
\hline H35 & 722 & 3558 & 396 & 24 \\
\hline H36 & 297 & 1867 & 35 & 29 \\
\hline H37 & 843 & -136 & 124 & 30 \\
\hline
\end{tabular}




\begin{tabular}{|c|c|c|c|c|}
\hline H38 & 1821 & -484 & 579 & 34 \\
\hline H39 & 2256 & 1212 & 942 & 24 \\
\hline H41 & 5350 & 1047 & 1155 & 22 \\
\hline $\mathrm{H} 42$ & 4678 & 878 & 1676 & 26 \\
\hline $\mathrm{H} 43$ & 5287 & -8 & 2182 & 24 \\
\hline H44 & 7967 & -1761 & 2758 & 29 \\
\hline H45 & 9309 & -2024 & 2669 & 32 \\
\hline H46 & 9777 & -1832 & 2100 & 23 \\
\hline H49A & 6360 & -2984 & 2158 & 36 \\
\hline H49B & 5754 & -2299 & 2407 & 36 \\
\hline $\mathrm{H} 49 \mathrm{C}$ & 6577 & -2846 & 2575 & 36 \\
\hline H50A & 6887 & -706 & 2839 & 34 \\
\hline H50B & 6084 & -118 & 2661 & 34 \\
\hline $\mathrm{H} 50 \mathrm{C}$ & 6920 & 519 & 2590 & 34 \\
\hline H56 & 5720 & 634 & 404 & 28 \\
\hline H57 & 5168 & 2385 & 96 & 33 \\
\hline H58 & 5638 & 4408 & 217 & 32 \\
\hline H59 & 6663 & 4739 & 651 & 32 \\
\hline H60 & 7224 & 2995 & 959 & 24 \\
\hline H62 & 5849 & -1781 & 920 & 25 \\
\hline H63 & 5585 & -3408 & 508 & 28 \\
\hline H64 & 6353 & -3589 & 18 & 31 \\
\hline H65 & 7379 & -2162 & -68 & 34 \\
\hline H66 & 7660 & -563 & 349 & 33 \\
\hline H68 & 8698 & -3824 & 1573 & 25 \\
\hline H69 & 8567 & -5672 & 1238 & 31 \\
\hline H70 & 8629 & -5603 & 626 & 31 \\
\hline H71 & 8817 & -3677 & 342 & 40 \\
\hline H72 & 8929 & -1794 & 673 & 31 \\
\hline H74 & 10397 & -2842 & 1378 & 24 \\
\hline H75 & 11764 & -2491 & 1440 & 28 \\
\hline H76 & 12262 & -462 & 1556 & 25 \\
\hline H77 & 11407 & 1237 & 1618 & 27 \\
\hline H78 & 10036 & 928 & 1529 & 22 \\
\hline
\end{tabular}


Table S24. Torsion angles $\left[{ }^{\circ}\right]$ for neivf02.

\begin{tabular}{|c|c|c|c|}
\hline C22-P1-C1-C2 & $-100.1(4)$ & C14-C9-C13-C12 & $15.2(5)$ \\
\hline C16-P1-C1-C2 & $9.5(4)$ & C10-C9-C13-C4 & $74.6(5)$ \\
\hline $\mathrm{Fe} 1-\mathrm{P} 1-\mathrm{C} 1-\mathrm{C} 2$ & $129.9(3)$ & C11-C9-C13-C4 & $-45.5(5)$ \\
\hline C22-P1-C1-C12 & $85.4(3)$ & C14-C9-C13-C4 & $-165.8(4)$ \\
\hline C16-P1-C1-C12 & $-165.1(3)$ & C6-C5-C14-C15 & $1.6(6)$ \\
\hline Fe1-P1-C1-C12 & $-44.6(3)$ & C6-C5-C14-C9 & $179.5(4)$ \\
\hline $\mathrm{C} 12-\mathrm{C} 1-\mathrm{C} 2-\mathrm{C} 3$ & $-0.9(6)$ & C13-C9-C14-C15 & $-14.2(5)$ \\
\hline $\mathrm{P} 1-\mathrm{C} 1-\mathrm{C} 2-\mathrm{C} 3$ & $-175.4(3)$ & C10-C9-C14-C15 & $105.3(4)$ \\
\hline $\mathrm{C} 1-\mathrm{C} 2-\mathrm{C} 3-\mathrm{C} 4$ & $1.4(7)$ & C11-C9-C14-C15 & $-134.2(4)$ \\
\hline C2-C3-C4-C13 & $0.1(7)$ & C13-C9-C14-C5 & $168.1(4)$ \\
\hline C14-C5-C6-C7 & $0.5(6)$ & C10-C9-C14-C5 & $-72.4(5)$ \\
\hline C5-C6-C7-C8 & $-0.5(6)$ & C11-C9-C14-C5 & $48.1(5)$ \\
\hline C6-C7-C8-C15 & $-1.6(6)$ & C12-O1-C15-C14 & $19.0(5)$ \\
\hline C6-C7-C8-P2 & 172.1(3) & $\mathrm{C} 12-\mathrm{O} 1-\mathrm{C} 15-\mathrm{C} 8$ & $-161.2(3)$ \\
\hline C28-P2-C8-C7 & $91.0(4)$ & C5-C14-C15-O1 & $175.9(3)$ \\
\hline C34-P2-C8-C7 & $-16.4(4)$ & C9-C14-C15-O1 & $-2.0(6)$ \\
\hline Fe1-P2-C8-C7 & $-136.9(3)$ & C5-C14-C15-C8 & $-3.9(6)$ \\
\hline C28-P2-C8-C15 & $-95.4(3)$ & C9-C14-C15-C8 & $178.2(4)$ \\
\hline C34-P2-C8-C15 & $157.2(3)$ & C7-C8-C15-O1 & $-175.9(3)$ \\
\hline Fe1-P2-C8-C15 & $36.7(3)$ & $\mathrm{P} 2-\mathrm{C} 8-\mathrm{C} 15-\mathrm{O} 1$ & $10.0(4)$ \\
\hline C15-O1-C12-C13 & $-18.1(5)$ & C7-C8-C15-C14 & $3.9(6)$ \\
\hline C15-O1-C12-C1 & $160.1(3)$ & P2-C8-C15-C14 & $-170.2(3)$ \\
\hline $\mathrm{C} 2-\mathrm{C} 1-\mathrm{C} 12-\mathrm{C} 13$ & $-1.4(6)$ & C1-P1-C16-C17 & $-92.3(4)$ \\
\hline P1-C1-C12-C13 & 173.6(3) & C22-P1-C16-C17 & $18.6(4)$ \\
\hline $\mathrm{C} 2-\mathrm{C} 1-\mathrm{C} 12-\mathrm{O} 1$ & $-179.5(3)$ & Fe1-P1-C16-C17 & $150.5(3)$ \\
\hline P1-C1-C12-O1 & $-4.5(5)$ & C1-P1-C16-C21 & $88.6(3)$ \\
\hline $\mathrm{O} 1-\mathrm{C} 12-\mathrm{C} 13-\mathrm{C} 4$ & $-179.2(4)$ & C22-P1-C16-C21 & $-160.5(3)$ \\
\hline $\mathrm{C} 1-\mathrm{C} 12-\mathrm{C} 13-\mathrm{C} 4$ & $2.9(6)$ & Fe1-P1-C16-C21 & $-28.6(3)$ \\
\hline O1-C12-C13-C9 & $-0.1(6)$ & $\mathrm{C} 21-\mathrm{C} 16-\mathrm{C} 17-\mathrm{C} 18$ & $1.0(6)$ \\
\hline C1-C12-C13-C9 & $-178.1(4)$ & P1-C16-C17-C18 & $-178.1(3)$ \\
\hline C3-C4-C13-C12 & $-2.2(6)$ & C16-C17-C18-C19 & $-1.9(6)$ \\
\hline C3-C4-C13-C9 & $178.7(4)$ & C17-C18-C19-C20 & $1.6(6)$ \\
\hline C10-C9-C13-C12 & $-104.5(4)$ & C18-C19-C20-C21 & $-0.4(6)$ \\
\hline C11-C9-C13-C12 & $135.5(4)$ & C19-C20-C21-C16 & $-0.5(6)$ \\
\hline
\end{tabular}




\begin{tabular}{|c|c|c|c|}
\hline C17-C16-C21-C20 & $0.2(6)$ & C39-C34-C35-C36 & $-0.9(6)$ \\
\hline P1-C16-C21-C20 & $179.3(3)$ & P2-C34-C35-C36 & 174.1(3) \\
\hline C1-P1-C22-C27 & $-164.5(3)$ & C34-C35-C36-C37 & $0.4(6)$ \\
\hline C16-P1-C22-C27 & $85.9(3)$ & C35-C36-C37-C38 & $0.2(7)$ \\
\hline Fe1-P1-C22-C27 & $-41.8(4)$ & C36-C37-C38-C39 & $-0.4(7)$ \\
\hline C1-P1-C22-C23 & $17.8(4)$ & C35-C34-C39-C38 & $0.8(6)$ \\
\hline C16-P1-C22-C23 & $-91.8(3)$ & P2-C34-C39-C38 & $-174.5(3)$ \\
\hline Fe1-P1-C22-C23 & $140.5(3)$ & C37-C38-C39-C34 & $-0.1(7)$ \\
\hline C27-C22-C23-C24 & $-1.4(6)$ & C61-P3-C40-C41 & $-91.9(4)$ \\
\hline P1-C22-C23-C24 & $176.3(3)$ & C55-P3-C40-C41 & $16.3(4)$ \\
\hline $\mathrm{C} 22-\mathrm{C} 23-\mathrm{C} 24-\mathrm{C} 25$ & $1.8(6)$ & Fe2-P3-C40-C41 & $136.5(3)$ \\
\hline $\mathrm{C} 23-\mathrm{C} 24-\mathrm{C} 25-\mathrm{C} 26$ & $-1.1(6)$ & C61-P3-C40-C51 & $94.2(3)$ \\
\hline C24-C25-C26-C27 & $0.1(7)$ & C55-P3-C40-C51 & $-157.5(3)$ \\
\hline C25-C26-C27-C22 & $0.2(7)$ & Fe2-P3-C40-C51 & $-37.3(3)$ \\
\hline C23-C22-C27-C26 & $0.4(6)$ & $\mathrm{C} 51-\mathrm{C} 40-\mathrm{C} 41-\mathrm{C} 42$ & $0.9(6)$ \\
\hline P1-C22-C27-C26 & $-177.4(3)$ & P3-C40-C41-C42 & $-173.0(3)$ \\
\hline C8-P2-C28-C29 & $-9.5(4)$ & $\mathrm{C} 40-\mathrm{C} 41-\mathrm{C} 42-\mathrm{C} 43$ & $2.1(6)$ \\
\hline C34-P2-C28-C29 & $101.2(4)$ & C41-C42-C43-C52 & $-2.6(6)$ \\
\hline Fe1-P2-C28-C29 & $-135.5(3)$ & C53-C44-C45-C46 & $-0.7(7)$ \\
\hline C8-P2-C28-C33 & $174.7(3)$ & C44-C45-C46-C47 & $0.1(7)$ \\
\hline C34-P2-C28-C33 & $-74.6(3)$ & C45-C46-C47-C54 & $-0.2(6)$ \\
\hline Fe1-P2-C28-C33 & $48.7(4)$ & C45-C46-C47-P4 & $174.9(3)$ \\
\hline C33-C28-C29-C30 & $-0.3(6)$ & C73-P4-C47-C46 & $-10.9(4)$ \\
\hline P2-C28-C29-C30 & $-176.0(3)$ & C67-P4-C47-C46 & $98.6(4)$ \\
\hline C28-C29-C30-C31 & $1.2(7)$ & Fe2-P4-C47-C46 & $-131.1(3)$ \\
\hline C29-C30-C31-C32 & $-0.8(7)$ & C73-P4-C47-C54 & $164.2(3)$ \\
\hline C30-C31-C32-C33 & $-0.5(7)$ & C67-P4-C47-C54 & $-86.3(3)$ \\
\hline C31-C32-C33-C28 & $1.4(7)$ & Fe2-P4-C47-C54 & $44.0(3)$ \\
\hline C29-C28-C33-C32 & $-1.0(6)$ & C54-O2-C51-C52 & $-20.7(5)$ \\
\hline P2-C28-C33-C32 & $175.0(3)$ & $\mathrm{C} 54-\mathrm{O} 2-\mathrm{C} 51-\mathrm{C} 40$ & $159.7(3)$ \\
\hline C28-P2-C34-C39 & $151.6(3)$ & C41-C40-C51-C52 & $-3.7(6)$ \\
\hline C8-P2-C34-C39 & $-97.1(3)$ & P3-C40-C51-C52 & $170.5(3)$ \\
\hline Fe1-P2-C34-C39 & $22.3(3)$ & $\mathrm{C} 41-\mathrm{C} 40-\mathrm{C} 51-\mathrm{O} 2$ & $175.9(3)$ \\
\hline C28-P2-C34-C35 & $-23.4(4)$ & $\mathrm{P} 3-\mathrm{C} 40-\mathrm{C} 51-\mathrm{O} 2$ & $-9.9(5)$ \\
\hline C8-P2-C34-C35 & $87.8(3)$ & $\mathrm{O} 2-\mathrm{C} 51-\mathrm{C} 52-\mathrm{C} 43$ & $-176.4(3)$ \\
\hline Fe1-P2-C34-C35 & $-152.7(3)$ & C40-C51-C52-C43 & $3.2(6)$ \\
\hline
\end{tabular}




\begin{tabular}{|c|c|c|c|}
\hline O2-C51-C52-C48 & $3.0(6)$ & C55-C56-C57-C58 & $0.3(6)$ \\
\hline C40-C51-C52-C48 & $-177.4(4)$ & C56-C57-C58-C59 & $-0.1(7)$ \\
\hline C42-C43-C52-C51 & $0.0(6)$ & C57-C58-C59-C60 & $0.3(7)$ \\
\hline C42-C43-C52-C48 & $-179.3(4)$ & C56-C55-C60-C59 & $0.8(6)$ \\
\hline C53-C48-C52-C51 & $13.2(5)$ & P3-C55-C60-C59 & $-179.5(3)$ \\
\hline C49-C48-C52-C51 & $-106.1(4)$ & C58-C59-C60-C55 & $-0.6(6)$ \\
\hline C50-C48-C52-C51 & $133.2(4)$ & C55-P3-C61-C62 & $-99.8(4)$ \\
\hline C53-C48-C52-C43 & $-167.5(4)$ & C40-P3-C61-C62 & $9.8(4)$ \\
\hline C49-C48-C52-C43 & $73.2(5)$ & Fe2-P3-C61-C62 & $136.4(3)$ \\
\hline C50-C48-C52-C43 & $-47.5(5)$ & C55-P3-C61-C66 & $75.6(4)$ \\
\hline C45-C44-C53-C54 & $1.2(6)$ & C40-P3-C61-C66 & $-174.7(3)$ \\
\hline C45-C44-C53-C48 & $-178.0(4)$ & Fe2-P3-C61-C66 & $-48.2(4)$ \\
\hline C52-C48-C53-C54 & $-13.3(5)$ & C66-C61-C62-C63 & $0.4(6)$ \\
\hline C49-C48-C53-C54 & $106.2(4)$ & P3-C61-C62-C63 & $175.8(3)$ \\
\hline C50-C48-C53-C54 & $-134.1(4)$ & C61-C62-C63-C64 & $-0.5(6)$ \\
\hline C52-C48-C53-C44 & $165.9(4)$ & C62-C63-C64-C65 & $-0.1(7)$ \\
\hline C49-C48-C53-C44 & $-74.7(5)$ & C63-C64-C65-C66 & $0.8(7)$ \\
\hline C50-C48-C53-C44 & $45.0(5)$ & C64-C65-C66-C61 & $-0.9(7)$ \\
\hline $\mathrm{C} 44-\mathrm{C} 53-\mathrm{C} 54-\mathrm{O} 2$ & $178.0(4)$ & C62-C61-C66-C65 & $0.3(7)$ \\
\hline C48-C53-C54-O2 & $-2.8(6)$ & P3-C61-C66-C65 & $-175.3(4)$ \\
\hline C44-C53-C54-C47 & $-1.3(6)$ & C73-P4-C67-C68 & $93.7(3)$ \\
\hline C48-C53-C54-C47 & $177.9(4)$ & C47-P4-C67-C68 & $-15.0(4)$ \\
\hline C51-O2-C54-C53 & $20.7(5)$ & Fe2-P4-C67-C68 & $-138.9(3)$ \\
\hline C51-O2-C54-C47 & $-160.0(3)$ & C73-P4-C67-C72 & $-84.1(3)$ \\
\hline C46-C47-C54-C53 & $0.8(6)$ & C47-P4-C67-C72 & $167.2(3)$ \\
\hline P4-C47-C54-C53 & $-174.6(3)$ & Fe2-P4-C67-C72 & $43.3(3)$ \\
\hline $\mathrm{C} 46-\mathrm{C} 47-\mathrm{C} 54-\mathrm{O} 2$ & $-178.5(3)$ & C72-C67-C68-C69 & $1.0(6)$ \\
\hline $\mathrm{P} 4-\mathrm{C} 47-\mathrm{C} 54-\mathrm{O} 2$ & $6.0(5)$ & P4-C67-C68-C69 & $-176.7(3)$ \\
\hline C61-P3-C55-C60 & $-155.6(3)$ & C67-C68-C69-C70 & $-0.1(6)$ \\
\hline C40-P3-C55-C60 & $93.2(3)$ & C68-C69-C70-C71 & $-0.3(7)$ \\
\hline Fe2-P3-C55-C60 & $-26.6(4)$ & C69-C70-C71-C72 & $-0.2(7)$ \\
\hline C61-P3-C55-C56 & $24.0(4)$ & C70-C71-C72-C67 & $1.2(7)$ \\
\hline C40-P3-C55-C56 & $-87.2(4)$ & C68-C67-C72-C71 & $-1.6(6)$ \\
\hline Fe2-P3-C55-C56 & $153.0(3)$ & P4-C67-C72-C71 & $176.3(3)$ \\
\hline C60-C55-C56-C57 & $-0.6(6)$ & C67-P4-C73-C78 & $158.3(3)$ \\
\hline P3-C55-C56-C57 & $179.8(3)$ & C47-P4-C73-C78 & $-91.1(3)$ \\
\hline
\end{tabular}




$\begin{array}{lclc}\text { Fe2-P4-C73-C78 } & 26.6(4) & \text { C73-C74-C75-C76 } & 0.2(6) \\ \text { C67-P4-C73-C74 } & -18.9(4) & \text { C74-C75-C76-C77 } & 0.1(7) \\ \text { C47-P4-C73-C74 } & 91.7(4) & \text { C75-C76-C77-C78 } & -1.7(6) \\ \text { Fe2-P4-C73-C74 } & -150.6(3) & \text { C74-C73-C78-C77 } & -2.8(6) \\ \text { C78-C73-C74-C75 } & 1.1(6) & \text { P4-C73-C78-C77 } & 179.9(3) \\ \text { P4-C73-C74-C75 } & 178.2(3) & \text { C76-C77-C78-C73 } & 3.1(6)\end{array}$




\section{$3.5[\mathrm{FeBr}(\mathrm{SciOPP})]_{2}$}

REFERENCE NUMBER: neijk39

CRYSTAL STRUCTURE REPORT

$$
\mathrm{C}_{128} \mathrm{H}_{186} \mathrm{Br}_{2} \mathrm{Fe}_{2} \mathrm{O} \mathrm{P} \mathrm{P}_{4}
$$

or

$[(\mathrm{SciOPP}) \mathrm{Fe}(\mu-\mathrm{Br})]_{2} \cdot \mathrm{Et}_{2} \mathrm{O}$

Report prepared for:

A. Shaps, J. Kneebone, Prof. M. Neidig

February 11, 2015

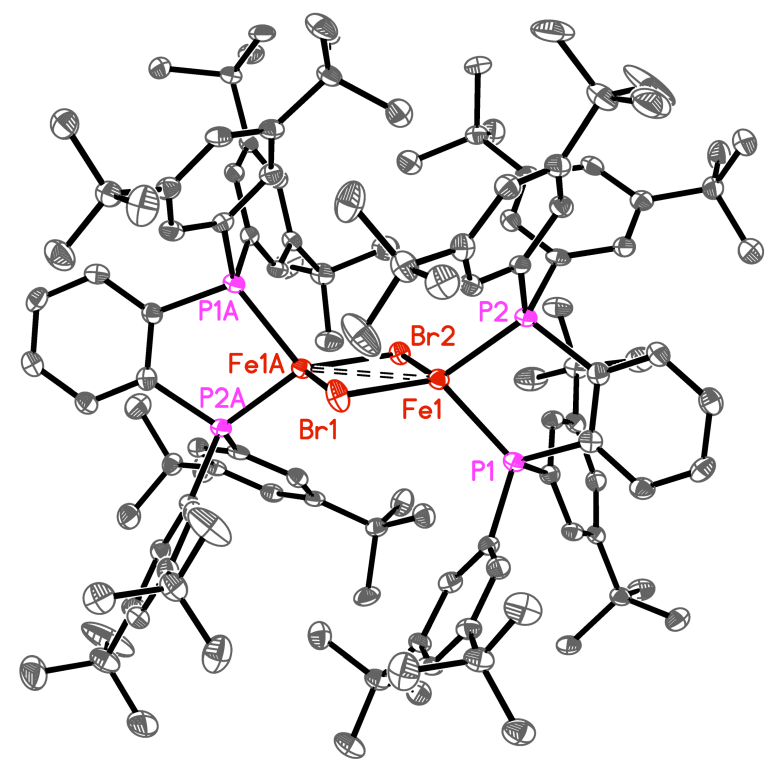

William W. Brennessel

X-ray Crystallographic Facility

Department of Chemistry, University of Rochester

120 Trustee Road

Rochester, NY 14627 


\section{Data collection}

A crystal $\left(0.20 \times 0.18 \times 0.14 \mathrm{~mm}^{3}\right)$ was placed onto the tip of a thin glass optical fiber and mounted on a Bruker SMART APEX II CCD platform diffractometer for a data collection at $100.0(5) \mathrm{K}^{1}$ A preliminary set of cell constants and an orientation matrix were calculated from reflections harvested from three orthogonal wedges of reciprocal space. The full data collection was carried out using MoKa radiation (graphite monochromator) with a frame time of 60 seconds and a detector distance of $4.02 \mathrm{~cm}$. A randomly oriented region of reciprocal space was surveyed: five major sections of frames were collected with $0.50^{\circ}$ steps in $\omega$ at five different $\phi$ settings and a detector position of $-38^{\circ}$ in $2 \theta$. The intensity data were corrected for absorption. ${ }^{2}$ Final cell constants were calculated from the xyz centroids of 4010 strong reflections from the actual data collection after integration. ${ }^{3}$ See Table 1 for additional crystal and refinement information.

\section{Structure solution and refinement}

The structure was solved using SIR20114 and refined using SHELXL-2014/7.5 The space group $P 2 / n$ was determined based on systematic absences and intensity statistics. A direct-methods solution was calculated which provided most non-hydrogen atoms from the E-map. Full-matrix least squares / difference Fourier cycles were performed which located the remaining non-hydrogen atoms. All non-hydrogen atoms were refined with anisotropic displacement parameters. All hydrogen atoms were placed in ideal positions and refined as riding atoms with relative isotropic displacement parameters. The final full matrix least squares refinement converged to $R 1=0.0527$ $\left(F^{2}, I>2 \sigma(I)\right)$ and $w R 2=0.1096\left(F^{2}\right.$, all data $)$.

\section{Structure description}

The structure is the one suggested. The asymmetric unit contains the metal center and one SciOPP ligand in general positions, the two bridging bromido ligands on a crystallographic two-fold axis, and one co-crystallized diethyl ether molecule on a crystallographic inversion center. One tert-butyl group (and its symmetry equivalent) is modeled as disordered over two positions (0.60:0.40) and the diethyl ether molecule is modeled as disordered over the crystallographic inversion center (0.50:0.50).

Unless noted otherwise all structural diagrams containing thermal displacement ellipsoids are drawn at the $50 \%$ probability level.

Data collection, structure solution, and structure refinement were conducted at the X-ray Crystallographic Facility, B51 Hutchison Hall, Department of Chemistry, University of Rochester. All publications arising from this report MUST either 1) include William W. Brennessel as a coauthor or 2) acknowledge William W. Brennessel and the Xray Crystallographic Facility of the Department of Chemistry at the University of Rochester. 
1 APEX3, version 2015.5-2; Bruker AXS: Madison, WI, 2015.

2 Sheldrick, G. M. SADABS, version 2014/5; J. Appl. Cryst. 2015, 48, 3-10.

3 SAINT, version 8.34A; Bruker AXS: Madison, WI, 2013.

4 Burla, M. C.; Caliandro, R.; Camalli, M.; Carrozzini, B.; Cascarano, G. L.; Giacovazzo, C.; Mallamo, M.; Mazzone, A.; Polidori, G.; Spagna, R. SIR2011, version 1.0; J. Appl. Cryst. 2012, 45, 357-361.

5 Sheldrick, G. M. SHELXL-2014/7; Acta. Cryst. 2015, C71, 3-8.

Some equations of interest:

$$
\begin{gathered}
R_{\mathrm{int}}=\Sigma\left|F_{\mathrm{o}}{ }^{2}-<F_{\mathrm{o}}{ }^{2}>\right| / \Sigma\left|F_{\mathrm{o}}{ }^{2}\right| \\
R 1=\Sigma|| F_{\mathrm{o}}|-| F_{\mathrm{c}}|/ \Sigma| F_{\mathrm{o}} \mid \\
w R 2=\left[\Sigma\left[w\left(F_{\mathrm{o}}{ }^{2}-F_{\mathrm{c}}{ }^{2}\right)^{2}\right] / \Sigma\left[w\left(F_{\mathrm{o}}{ }^{2}\right)^{2}\right]\right]^{1 / 2} \\
\text { where } w=1 /\left[\sigma^{2}\left(F_{\mathrm{o}}{ }^{2}\right)+(a P)^{2}+b P\right] \text { and } \\
P=1 / 3 \max \left(0, F_{\mathrm{o}}{ }^{2}\right)+2 / 3 F_{\mathrm{c}}{ }^{2} \\
\text { GOF }=S=\left[\Sigma\left[w\left(F_{\mathrm{o}}{ }^{2}-F_{\mathrm{c}}{ }^{2}\right)^{2}\right] /(m-n)\right]^{1 / 2}
\end{gathered}
$$

where $m=$ number of reflections and $n=$ number of parameters

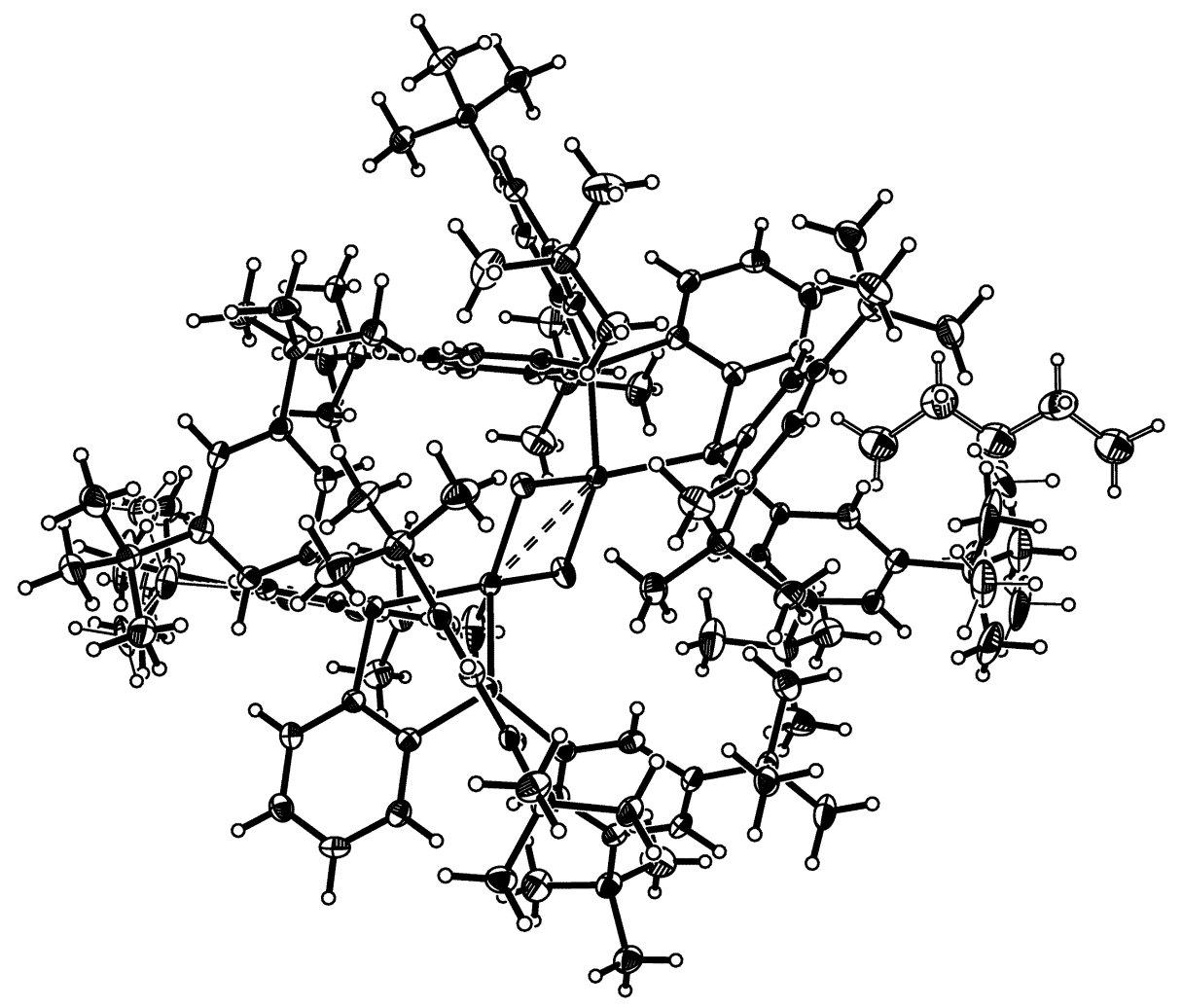



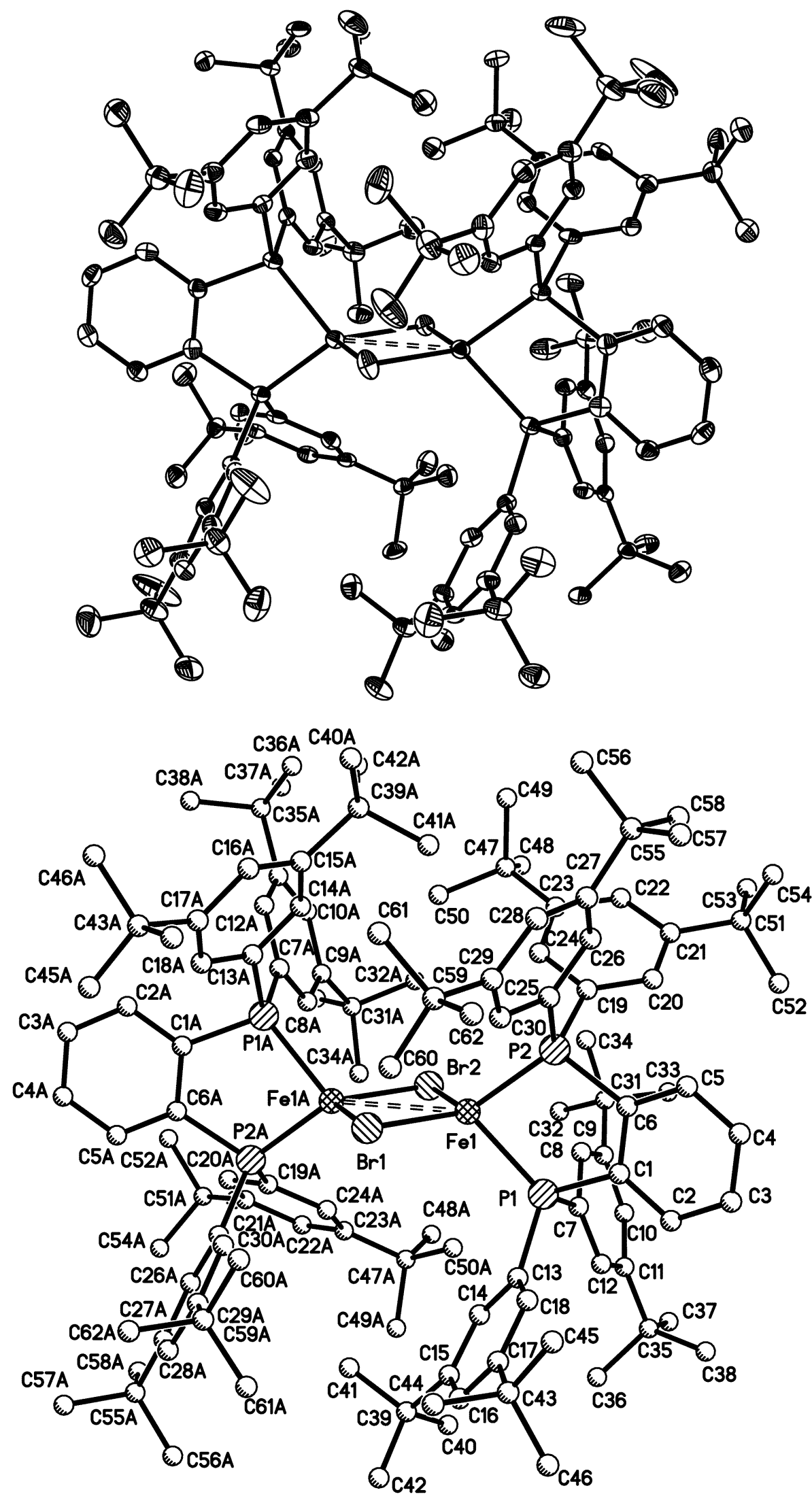

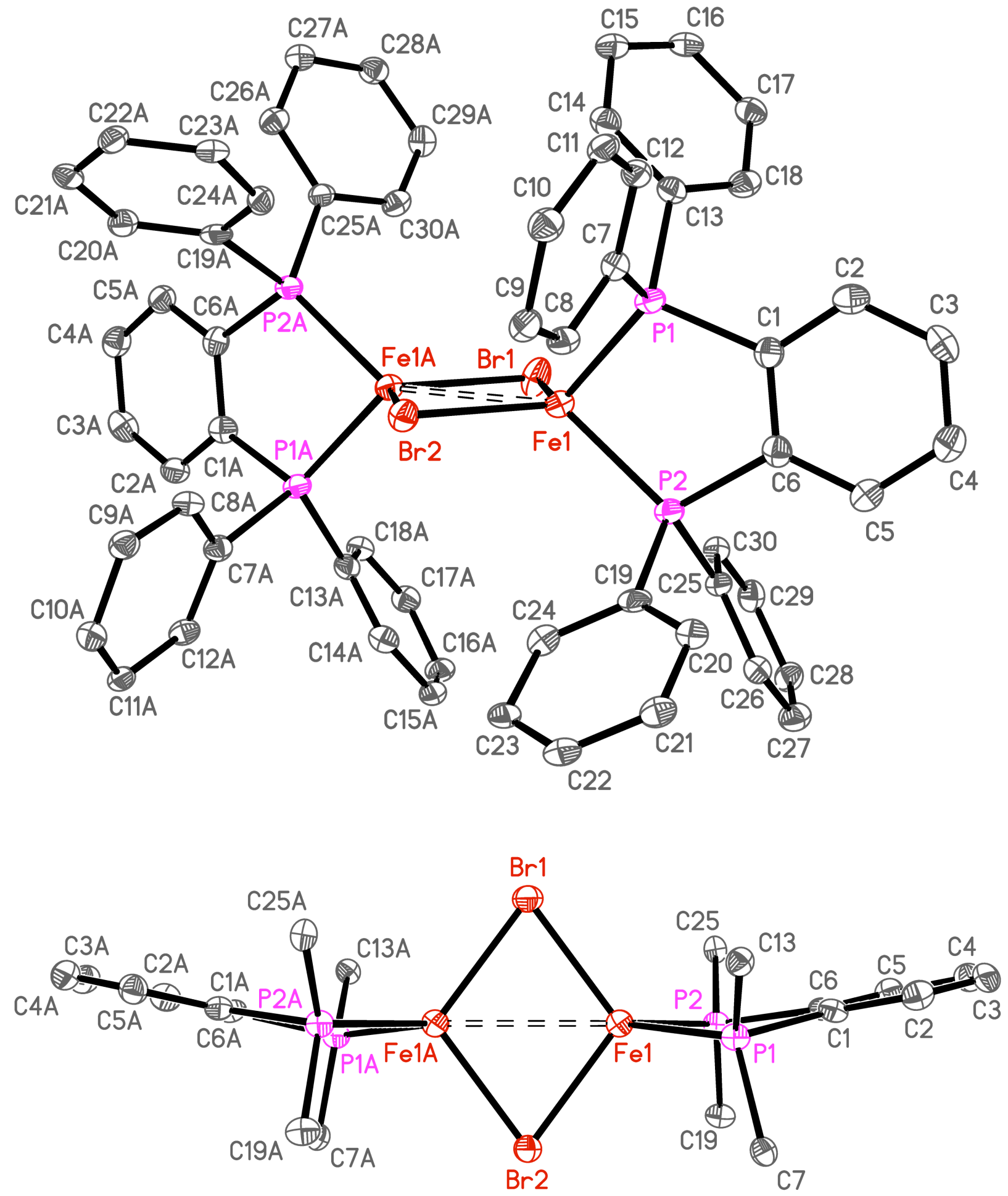
Table S25. Crystal data and structure refinement for neijk39.

\begin{tabular}{|c|c|c|}
\hline Identification code & \multicolumn{2}{|l|}{ neijk39 } \\
\hline Empirical formula & \multicolumn{2}{|c|}{$\mathrm{C} 128 \mathrm{H} 186 \mathrm{Br} 2 \mathrm{Fe} 2 \mathrm{O}$ P4 } \\
\hline Formula weight & \multicolumn{2}{|l|}{2136.16} \\
\hline Temperature & \multicolumn{2}{|l|}{$100.0(5) \mathrm{K}$} \\
\hline Wavelength & \multicolumn{2}{|l|}{$0.71073 \AA$} \\
\hline Crystal system & \multicolumn{2}{|l|}{ monoclinic } \\
\hline Space group & \multicolumn{2}{|l|}{$P 2 / n$} \\
\hline \multirow[t]{3}{*}{ Unit cell dimensions } & $a=14.5951(11) \AA$ & $\alpha=90^{\circ}$ \\
\hline & $b=14.2424(11) \AA$ & $\beta=103.2648(14)^{\circ}$ \\
\hline & $c=30.373(2) \AA$ & $\gamma=90^{\circ}$ \\
\hline Volume & \multicolumn{2}{|l|}{$6145.1(8) \AA^{3}$} \\
\hline$Z$ & \multicolumn{2}{|l|}{2} \\
\hline Density (calculated) & \multicolumn{2}{|l|}{$1.154 \mathrm{Mg} / \mathrm{m}^{3}$} \\
\hline Absorption coefficient & \multicolumn{2}{|l|}{$0.984 \mathrm{~mm}^{-1}$} \\
\hline$F(000)$ & \multicolumn{2}{|l|}{2288} \\
\hline Crystal color, morphology & \multicolumn{2}{|l|}{ dark orange, plate } \\
\hline Crystal size & \multicolumn{2}{|c|}{$0.20 \times 0.18 \times 0.14 \mathrm{~mm}^{3}$} \\
\hline Theta range for data collection & \multicolumn{2}{|l|}{1.986 to $26.409^{\circ}$} \\
\hline Index ranges & \multicolumn{2}{|c|}{$-18 \leq h \leq 18,-17 \leq k \leq 17,-37 \leq l \leq 37$} \\
\hline Reflections collected & \multicolumn{2}{|l|}{74990} \\
\hline Independent reflections & \multicolumn{2}{|c|}{$12610[R(\mathrm{int})=0.1312]$} \\
\hline Observed reflections & \multicolumn{2}{|l|}{7743} \\
\hline Completeness to theta $=26.373^{\circ}$ & \multicolumn{2}{|l|}{$100.0 \%$} \\
\hline Absorption correction & \multicolumn{2}{|l|}{ Multi-scan } \\
\hline Max. and min. transmission & \multicolumn{2}{|l|}{0.7454 and 0.6548} \\
\hline Refinement method & \multicolumn{2}{|c|}{ Full-matrix least-squares on $F^{2}$} \\
\hline Data / restraints / parameters & \multicolumn{2}{|l|}{$12610 / 46 / 677$} \\
\hline Goodness-of-fit on $F^{2}$ & \multicolumn{2}{|l|}{1.021} \\
\hline Final $R$ indices $[I>2 \operatorname{sigma}(I)]$ & \multicolumn{2}{|c|}{$R 1=0.0527, w R 2=0.0932$} \\
\hline$R$ indices (all data) & \multicolumn{2}{|c|}{$R 1=0.1085, w R 2=0.1096$} \\
\hline Largest diff. peak and hole & \multicolumn{2}{|c|}{0.448 and -0.421 e. $\AA^{-3}$} \\
\hline
\end{tabular}


Table S26. Atomic coordinates $\left(\times 10^{4}\right)$ and equivalent isotropic displacement parameters $\left(\AA^{2} \times 10^{3}\right)$ for neijk39. $U_{e q}$ is defined as one third of the trace of the orthogonalized $U_{i j}$ tensor.

\begin{tabular}{|c|c|c|c|c|}
\hline & $\mathrm{x}$ & $\mathrm{y}$ & $\mathrm{z}$ & $\mathrm{U}_{\mathrm{eq}}$ \\
\hline Fe1 & $3248(1)$ & 1841(1) & 2254(1) & $17(1)$ \\
\hline $\mathrm{Br} 1$ & 2500 & $3216(1)$ & 2500 & $27(1)$ \\
\hline $\mathrm{Br} 2$ & 2500 & $457(1)$ & 2500 & $20(1)$ \\
\hline P1 & $4835(1)$ & $1698(1)$ & 2476(1) & $17(1)$ \\
\hline $\mathrm{P} 2$ & $3496(1)$ & $1836(1)$ & 1540(1) & $16(1)$ \\
\hline $\mathrm{C} 1$ & $5366(2)$ & 1978(2) & 2000(1) & $18(1)$ \\
\hline $\mathrm{C} 2$ & $6328(2)$ & $2139(2)$ & 2046(1) & $21(1)$ \\
\hline C3 & 6689(3) & $2335(2)$ & 1672(1) & $24(1)$ \\
\hline $\mathrm{C} 4$ & 6093(3) & $2360(2)$ & $1246(1)$ & $24(1)$ \\
\hline C5 & 5141(3) & 2201(2) & 1195(1) & $21(1)$ \\
\hline C6 & $4765(2)$ & $2003(2)$ & 1568(1) & $18(1)$ \\
\hline C7 & $5317(2)$ & $511(2)$ & 2625(1) & $17(1)$ \\
\hline $\mathrm{C} 8$ & $4766(2)$ & $-224(2)$ & 2401(1) & $18(1)$ \\
\hline C9 & $5028(2)$ & $-1155(2)$ & 2484(1) & $19(1)$ \\
\hline $\mathrm{C} 10$ & $5881(2)$ & $-1327(2)$ & 2802(1) & $20(1)$ \\
\hline C11 & $6459(2)$ & $-610(2)$ & 3022(1) & $17(1)$ \\
\hline $\mathrm{C} 12$ & $6159(2)$ & $314(2)$ & 2927(1) & $17(1)$ \\
\hline $\mathrm{C} 13$ & $5436(2)$ & 2463(2) & 2941(1) & $17(1)$ \\
\hline $\mathrm{C} 14$ & $5445(2)$ & $2212(2)$ & $3379(1)$ & $19(1)$ \\
\hline C15 & $5760(2)$ & $2838(2)$ & 3743(1) & $19(1)$ \\
\hline $\mathrm{C} 16$ & 6071(2) & $3711(2)$ & $3642(1)$ & $21(1)$ \\
\hline C17 & $6057(2)$ & $4000(2)$ & 3201(1) & $20(1)$ \\
\hline $\mathrm{C} 18$ & $5737(2)$ & $3365(2)$ & 2852(1) & $21(1)$ \\
\hline C19 & 3161(2) & $742(2)$ & 1223(1) & $17(1)$ \\
\hline $\mathrm{C} 20$ & $3697(2)$ & $265(2)$ & $972(1)$ & $20(1)$ \\
\hline $\mathrm{C} 21$ & $3367(2)$ & $-556(2)$ & $734(1)$ & $19(1)$ \\
\hline $\mathrm{C} 22$ & 2473(2) & $-872(2)$ & $755(1)$ & $20(1)$ \\
\hline $\mathrm{C} 23$ & 1914(2) & $-423(2)$ & 1007(1) & $18(1)$ \\
\hline $\mathrm{C} 24$ & $2281(2)$ & $382(2)$ & 1241(1) & $18(1)$ \\
\hline $\mathrm{C} 25$ & $2976(2)$ & $2729(2)$ & $1120(1)$ & $17(1)$ \\
\hline $\mathrm{C} 26$ & 2803(2) & $2580(2)$ & $658(1)$ & $19(1)$ \\
\hline
\end{tabular}




\begin{tabular}{|c|c|c|c|c|}
\hline $\mathrm{C} 27$ & $2440(2)$ & $3293(3)$ & $350(1)$ & $22(1)$ \\
\hline $\mathrm{C} 28$ & $2234(2)$ & $4146(2)$ & $525(1)$ & $21(1)$ \\
\hline $\mathrm{C} 29$ & 2383(2) & $4323(2)$ & $988(1)$ & $21(1)$ \\
\hline $\mathrm{C} 30$ & 2758(2) & $3599(2)$ & 1281(1) & $19(1)$ \\
\hline C31 & 4423(3) & $-1974(2)$ & $2255(1)$ & $23(1)$ \\
\hline C32 & 4046(3) & $-2510(3)$ & $2618(1)$ & $37(1)$ \\
\hline C33 & 5011(3) & $-2630(3)$ & $2035(2)$ & $38(1)$ \\
\hline C34 & 3573(3) & $-1643(2)$ & $1895(1)$ & $29(1)$ \\
\hline C35 & 7403(2) & $-810(2)$ & $3354(1)$ & 19(1) \\
\hline C36 & 7391(3) & $-363(3)$ & $3814(1)$ & $29(1)$ \\
\hline C37 & 7601(3) & $-1861(3)$ & $3424(1)$ & $28(1)$ \\
\hline C38 & $8200(2)$ & $-371(3)$ & $3172(1)$ & $25(1)$ \\
\hline C39 & $5727(3)$ & $2536(2)$ & $4227(1)$ & $22(1)$ \\
\hline $\mathrm{C} 40$ & $6305(3)$ & 1633(2) & $4345(1)$ & $25(1)$ \\
\hline C41 & $4698(3)$ & 2341(3) & $4244(1)$ & $28(1)$ \\
\hline $\mathrm{C} 42$ & $6124(3)$ & $3293(3)$ & $4577(1)$ & $32(1)$ \\
\hline $\mathrm{C} 43$ & 6412(3) & 4983(2) & $3122(1)$ & $24(1)$ \\
\hline C44 & $5835(3)$ & $5715(3)$ & $3315(2)$ & $38(1)$ \\
\hline $\mathrm{C} 45$ & 6313(3) & $5182(3)$ & 2621(1) & $36(1)$ \\
\hline C46 & $7460(3)$ & $5065(3)$ & $3365(1)$ & $31(1)$ \\
\hline $\mathrm{C} 47$ & $936(2)$ & $-807(2)$ & 1014(1) & $20(1)$ \\
\hline C48 & $964(3)$ & $-1877(3)$ & 1081(1) & $30(1)$ \\
\hline C49 & $268(3)$ & $-571(3)$ & $555(1)$ & $29(1)$ \\
\hline C50 & $548(3)$ & $-370(3)$ & 1396(1) & $26(1)$ \\
\hline C51 & $3945(3)$ & $-1059(2)$ & $444(1)$ & $22(1)$ \\
\hline C52 & 4987(3) & $-1071(3)$ & $680(1)$ & $33(1)$ \\
\hline C53 & $3632(3)$ & $-2085(3)$ & $342(1)$ & $33(1)$ \\
\hline C54 & 3807(3) & $-532(3)$ & $-9(1)$ & $34(1)$ \\
\hline C55 & $2213(5)$ & $3140(5)$ & $-162(2)$ & $30(1)$ \\
\hline C56 & $1158(4)$ & $3285(6)$ & $-365(2)$ & $50(2)$ \\
\hline C57 & $2770(6)$ & $3854(6)$ & $-371(2)$ & $54(2)$ \\
\hline C58 & $2466(8)$ & $2142(5)$ & $-277(2)$ & $77(3)$ \\
\hline C55' & $2357(6)$ & $3125(7)$ & $-156(2)$ & $30(1)$ \\
\hline C56' & $3290(6)$ & 2796(9) & $-254(3)$ & $46(3)$ \\
\hline C57' & $1610(8)$ & 2363(9) & $-314(4)$ & $54(2)$ \\
\hline C58' & $2040(11)$ & 4009(8) & $-439(4)$ & $77(3)$ \\
\hline
\end{tabular}




\begin{tabular}{lllrl} 
C59 & $2106(3)$ & $5276(3)$ & $1149(1)$ & $27(1)$ \\
C60 & $2326(4)$ & $5350(3)$ & $1667(1)$ & $49(1)$ \\
C61 & $1046(3)$ & $5434(3)$ & $967(2)$ & $39(1)$ \\
C62 & $2648(3)$ & $6055(3)$ & $972(1)$ & $32(1)$ \\
C63 & $5014(16)$ & $5391(10)$ & $-731(5)$ & $53(3)$ \\
C64 & $5354(7)$ & $4663(6)$ & $-347(3)$ & $50(3)$ \\
O1 & $5040(20)$ & $5030(30)$ & $44(5)$ & $53(3)$ \\
C65 & $5388(7)$ & $4392(6)$ & $430(3)$ & $48(2)$ \\
C66 & $5002(16)$ & $4844(11)$ & $820(5)$ & $53(3)$ \\
\hline
\end{tabular}


Table S27. Bond lengths $[\AA]$ and angles $\left[{ }^{\circ}\right]$ for neijk39.

\begin{tabular}{|c|c|c|c|}
\hline $\mathrm{Fe}(1)-\mathrm{P}(1)$ & $2.2681(10)$ & $C(13)-C(14)$ & $1.377(5)$ \\
\hline $\mathrm{Fe}(1)-\mathrm{P}(2)$ & $2.2791(9)$ & $\mathrm{C}(13)-\mathrm{C}(18)$ & $1.404(5)$ \\
\hline $\mathrm{Fe}(1)-\mathrm{Br}(1)$ & $2.4411(7)$ & $C(14)-C(15)$ & $1.412(5)$ \\
\hline $\mathrm{Fe}(1)-\mathrm{Br}(2)$ & $2.4518(7)$ & $\mathrm{C}(14)-\mathrm{H}(14 \mathrm{~A})$ & 0.9500 \\
\hline $\mathrm{Fe}(1)-\mathrm{Fe}(1) \# 1$ & $2.9160(9)$ & $C(15)-C(16)$ & $1.382(5)$ \\
\hline $\operatorname{Br}(1)-\mathrm{Fe}(1) \# 1$ & $2.4410(7)$ & $C(15)-C(39)$ & $1.543(5)$ \\
\hline $\operatorname{Br}(2)-\mathrm{Fe}(1) \# 1$ & $2.4517(7)$ & $C(16)-C(17)$ & $1.398(5)$ \\
\hline $\mathrm{P}(1)-\mathrm{C}(1)$ & $1.834(3)$ & $\mathrm{C}(16)-\mathrm{H}(16 \mathrm{~A})$ & 0.9500 \\
\hline $\mathrm{P}(1)-\mathrm{C}(13)$ & $1.839(3)$ & $C(17)-C(18)$ & $1.389(5)$ \\
\hline $\mathrm{P}(1)-\mathrm{C}(7)$ & $1.846(3)$ & $C(17)-C(43)$ & $1.530(5)$ \\
\hline $\mathrm{P}(2)-\mathrm{C}(25)$ & $1.836(3)$ & $\mathrm{C}(18)-\mathrm{H}(18 \mathrm{~A})$ & 0.9500 \\
\hline $\mathrm{P}(2)-\mathrm{C}(19)$ & $1.838(3)$ & $C(19)-C(20)$ & $1.389(5)$ \\
\hline $\mathrm{P}(2)-\mathrm{C}(6)$ & $1.850(3)$ & $C(19)-C(24)$ & $1.396(5)$ \\
\hline $\mathrm{C}(1)-\mathrm{C}(2)$ & $1.398(5)$ & $C(20)-C(21)$ & $1.401(5)$ \\
\hline$C(1)-C(6)$ & $1.401(4)$ & $\mathrm{C}(20)-\mathrm{H}(20 \mathrm{~A})$ & 0.9500 \\
\hline $\mathrm{C}(2)-\mathrm{C}(3)$ & $1.388(5)$ & $C(21)-C(22)$ & $1.395(5)$ \\
\hline $\mathrm{C}(2)-\mathrm{H}(2 \mathrm{~A})$ & 0.9500 & $C(21)-C(51)$ & $1.530(5)$ \\
\hline $\mathrm{C}(3)-\mathrm{C}(4)$ & $1.384(5)$ & $C(22)-C(23)$ & $1.397(5)$ \\
\hline $\mathrm{C}(3)-\mathrm{H}(3 \mathrm{~A})$ & 0.9500 & $\mathrm{C}(22)-\mathrm{H}(22 \mathrm{~A})$ & 0.9500 \\
\hline$C(4)-C(5)$ & $1.382(5)$ & $C(23)-C(24)$ & $1.389(5)$ \\
\hline $\mathrm{C}(4)-\mathrm{H}(4 \mathrm{~A})$ & 0.9500 & $C(23)-C(47)$ & $1.534(5)$ \\
\hline$C(5)-C(6)$ & $1.394(5)$ & $\mathrm{C}(24)-\mathrm{H}(24 \mathrm{~A})$ & 0.9500 \\
\hline $\mathrm{C}(5)-\mathrm{H}(5 \mathrm{~A})$ & 0.9500 & $C(25)-C(26)$ & $1.386(4)$ \\
\hline$C(7)-C(12)$ & $1.384(4)$ & $C(25)-C(30)$ & $1.395(5)$ \\
\hline$C(7)-C(8)$ & $1.399(5)$ & $C(26)-C(27)$ & $1.400(5)$ \\
\hline $\mathrm{C}(8)-\mathrm{C}(9)$ & $1.387(5)$ & $\mathrm{C}(26)-\mathrm{H}(26 \mathrm{~A})$ & 0.9500 \\
\hline $\mathrm{C}(8)-\mathrm{H}(8 \mathrm{~A})$ & 0.9500 & $C(27)-C(28)$ & $1.386(5)$ \\
\hline$C(9)-C(10)$ & $1.410(5)$ & $C(27)-C(55)$ & $1.530(6)$ \\
\hline $\mathrm{C}(9)-\mathrm{C}(31)$ & $1.531(5)$ & $\mathrm{C}(27)-\mathrm{C}\left(55^{\prime}\right)$ & $1.533(7)$ \\
\hline $\mathrm{C}(10)-\mathrm{C}(11)$ & $1.394(5)$ & $\mathrm{C}(28)-\mathrm{C}(29)$ & $1.395(5)$ \\
\hline $\mathrm{C}(10)-\mathrm{H}(10 \mathrm{~A})$ & 0.9500 & $\mathrm{C}(28)-\mathrm{H}(28 \mathrm{~A})$ & 0.9500 \\
\hline$C(11)-C(12)$ & $1.397(5)$ & $C(29)-C(30)$ & $1.390(5)$ \\
\hline$C(11)-C(35)$ & $1.536(4)$ & $C(29)-C(59)$ & $1.528(5)$ \\
\hline $\mathrm{C}(12)-\mathrm{H}(12 \mathrm{~A})$ & 0.9500 & $\mathrm{C}(30)-\mathrm{H}(30 \mathrm{~A})$ & 0.9500 \\
\hline
\end{tabular}




\begin{tabular}{|c|c|c|c|}
\hline $\mathrm{C}(31)-\mathrm{C}(33)$ & $1.522(5)$ & $\mathrm{C}(43)-\mathrm{C}(45)$ & $1.523(5)$ \\
\hline $\mathrm{C}(31)-\mathrm{C}(34)$ & $1.528(5)$ & $\mathrm{C}(43)-\mathrm{C}(44)$ & $1.539(5)$ \\
\hline$C(31)-C(32)$ & $1.542(5)$ & $C(43)-C(46)$ & $1.543(5)$ \\
\hline $\mathrm{C}(32)-\mathrm{H}(32 \mathrm{~A})$ & 0.9800 & $\mathrm{C}(44)-\mathrm{H}(44 \mathrm{~A})$ & 0.9800 \\
\hline $\mathrm{C}(32)-\mathrm{H}(32 \mathrm{~B})$ & 0.9800 & $\mathrm{C}(44)-\mathrm{H}(44 \mathrm{~B})$ & 0.9800 \\
\hline $\mathrm{C}(32)-\mathrm{H}(32 \mathrm{C})$ & 0.9800 & $\mathrm{C}(44)-\mathrm{H}(44 \mathrm{C})$ & 0.9800 \\
\hline $\mathrm{C}(33)-\mathrm{H}(33 \mathrm{~A})$ & 0.9800 & $\mathrm{C}(45)-\mathrm{H}(45 \mathrm{~A})$ & 0.9800 \\
\hline $\mathrm{C}(33)-\mathrm{H}(33 \mathrm{~B})$ & 0.9800 & $\mathrm{C}(45)-\mathrm{H}(45 \mathrm{~B})$ & 0.9800 \\
\hline $\mathrm{C}(33)-\mathrm{H}(33 \mathrm{C})$ & 0.9800 & $\mathrm{C}(45)-\mathrm{H}(45 \mathrm{C})$ & 0.9800 \\
\hline $\mathrm{C}(34)-\mathrm{H}(34 \mathrm{~A})$ & 0.9800 & $\mathrm{C}(46)-\mathrm{H}(46 \mathrm{~A})$ & 0.9800 \\
\hline $\mathrm{C}(34)-\mathrm{H}(34 \mathrm{~B})$ & 0.9800 & $\mathrm{C}(46)-\mathrm{H}(46 \mathrm{~B})$ & 0.9800 \\
\hline $\mathrm{C}(34)-\mathrm{H}(34 \mathrm{C})$ & 0.9800 & $\mathrm{C}(46)-\mathrm{H}(46 \mathrm{C})$ & 0.9800 \\
\hline C(35)-C(37) & $1.529(5)$ & $C(47)-C(50)$ & $1.532(5)$ \\
\hline$C(35)-C(38)$ & $1.533(5)$ & $C(47)-C(48)$ & $1.536(5)$ \\
\hline$C(35)-C(36)$ & $1.538(5)$ & $\mathrm{C}(47)-\mathrm{C}(49)$ & $1.544(5)$ \\
\hline $\mathrm{C}(36)-\mathrm{H}(36 \mathrm{~A})$ & 0.9800 & $\mathrm{C}(48)-\mathrm{H}(48 \mathrm{~A})$ & 0.9800 \\
\hline $\mathrm{C}(36)-\mathrm{H}(36 \mathrm{~B})$ & 0.9800 & $\mathrm{C}(48)-\mathrm{H}(48 \mathrm{~B})$ & 0.9800 \\
\hline $\mathrm{C}(36)-\mathrm{H}(36 \mathrm{C})$ & 0.9800 & $\mathrm{C}(48)-\mathrm{H}(48 \mathrm{C})$ & 0.9800 \\
\hline $\mathrm{C}(37)-\mathrm{H}(37 \mathrm{~A})$ & 0.9800 & $\mathrm{C}(49)-\mathrm{H}(49 \mathrm{~A})$ & 0.9800 \\
\hline $\mathrm{C}(37)-\mathrm{H}(37 \mathrm{~B})$ & 0.9800 & $\mathrm{C}(49)-\mathrm{H}(49 \mathrm{~B})$ & 0.9800 \\
\hline $\mathrm{C}(37)-\mathrm{H}(37 \mathrm{C})$ & 0.9800 & $\mathrm{C}(49)-\mathrm{H}(49 \mathrm{C})$ & 0.9800 \\
\hline $\mathrm{C}(38)-\mathrm{H}(38 \mathrm{~A})$ & 0.9800 & $\mathrm{C}(50)-\mathrm{H}(50 \mathrm{~A})$ & 0.9800 \\
\hline $\mathrm{C}(38)-\mathrm{H}(38 \mathrm{~B})$ & 0.9800 & $\mathrm{C}(50)-\mathrm{H}(50 \mathrm{~B})$ & 0.9800 \\
\hline $\mathrm{C}(38)-\mathrm{H}(38 \mathrm{C})$ & 0.9800 & $\mathrm{C}(50)-\mathrm{H}(50 \mathrm{C})$ & 0.9800 \\
\hline C(39)-C(42) & $1.532(5)$ & $C(51)-C(52)$ & $1.525(5)$ \\
\hline C(39)-C(40) & $1.535(5)$ & $\mathrm{C}(51)-\mathrm{C}(54)$ & $1.540(5)$ \\
\hline $\mathrm{C}(39)-\mathrm{C}(41)$ & $1.540(5)$ & $\mathrm{C}(51)-\mathrm{C}(53)$ & $1.541(5)$ \\
\hline $\mathrm{C}(40)-\mathrm{H}(40 \mathrm{~A})$ & 0.9800 & $\mathrm{C}(52)-\mathrm{H}(52 \mathrm{~A})$ & 0.9800 \\
\hline $\mathrm{C}(40)-\mathrm{H}(40 \mathrm{~B})$ & 0.9800 & $\mathrm{C}(52)-\mathrm{H}(52 \mathrm{~B})$ & 0.9800 \\
\hline $\mathrm{C}(40)-\mathrm{H}(40 \mathrm{C})$ & 0.9800 & $\mathrm{C}(52)-\mathrm{H}(52 \mathrm{C})$ & 0.9800 \\
\hline $\mathrm{C}(41)-\mathrm{H}(41 \mathrm{~A})$ & 0.9800 & $\mathrm{C}(53)-\mathrm{H}(53 \mathrm{~A})$ & 0.9800 \\
\hline $\mathrm{C}(41)-\mathrm{H}(41 \mathrm{~B})$ & 0.9800 & $\mathrm{C}(53)-\mathrm{H}(53 \mathrm{~B})$ & 0.9800 \\
\hline $\mathrm{C}(41)-\mathrm{H}(41 \mathrm{C})$ & 0.9800 & $\mathrm{C}(53)-\mathrm{H}(53 \mathrm{C})$ & 0.9800 \\
\hline $\mathrm{C}(42)-\mathrm{H}(42 \mathrm{~A})$ & 0.9800 & $\mathrm{C}(54)-\mathrm{H}(54 \mathrm{~A})$ & 0.9800 \\
\hline $\mathrm{C}(42)-\mathrm{H}(42 \mathrm{~B})$ & 0.9800 & $\mathrm{C}(54)-\mathrm{H}(54 \mathrm{~B})$ & 0.9800 \\
\hline $\mathrm{C}(42)-\mathrm{H}(42 \mathrm{C})$ & 0.9800 & $\mathrm{C}(54)-\mathrm{H}(54 \mathrm{C})$ & 0.9800 \\
\hline
\end{tabular}




\begin{tabular}{|c|c|c|c|}
\hline$C(55)-C(57)$ & $1.529(6)$ & $C(63)-C(64)$ & $1.556(13)$ \\
\hline $\mathrm{C}(55)-\mathrm{C}(58)$ & $1.530(6)$ & $\mathrm{C}(63)-\mathrm{H}(63 \mathrm{~A})$ & 0.9800 \\
\hline$C(55)-C(56)$ & $1.535(6)$ & $\mathrm{C}(63)-\mathrm{H}(63 \mathrm{~B})$ & 0.9800 \\
\hline $\mathrm{C}(56)-\mathrm{H}(56 \mathrm{~A})$ & 0.9800 & $\mathrm{C}(63)-\mathrm{H}(63 \mathrm{C})$ & 0.9800 \\
\hline $\mathrm{C}(56)-\mathrm{H}(56 \mathrm{~B})$ & 0.9800 & $\mathrm{C}(64)-\mathrm{O}(1)$ & $1.46(3)$ \\
\hline $\mathrm{C}(56)-\mathrm{H}(56 \mathrm{C})$ & 0.9800 & $\mathrm{C}(64)-\mathrm{H}(64 \mathrm{~A})$ & 0.9900 \\
\hline $\mathrm{C}(57)-\mathrm{H}(57 \mathrm{~A})$ & 0.9800 & $\mathrm{C}(64)-\mathrm{H}(64 \mathrm{~B})$ & 0.9900 \\
\hline $\mathrm{C}(57)-\mathrm{H}(57 \mathrm{~B})$ & 0.9800 & $\mathrm{O}(1)-\mathrm{C}(65)$ & $1.47(2)$ \\
\hline $\mathrm{C}(57)-\mathrm{H}(57 \mathrm{C})$ & 0.9800 & $C(65)-C(66)$ & $1.562(13)$ \\
\hline $\mathrm{C}(58)-\mathrm{H}(58 \mathrm{~A})$ & 0.9800 & $\mathrm{C}(65)-\mathrm{H}(65 \mathrm{~A})$ & 0.9900 \\
\hline $\mathrm{C}(58)-\mathrm{H}(58 \mathrm{~B})$ & 0.9800 & $\mathrm{C}(65)-\mathrm{H}(65 \mathrm{~B})$ & 0.9900 \\
\hline $\mathrm{C}(58)-\mathrm{H}(58 \mathrm{C})$ & 0.9800 & $\mathrm{C}(66)-\mathrm{H}(66 \mathrm{~A})$ & 0.9800 \\
\hline $\mathrm{C}\left(55^{\prime}\right)-\mathrm{C}\left(56^{\prime}\right)$ & $1.531(7)$ & $\mathrm{C}(66)-\mathrm{H}(66 \mathrm{~B})$ & 0.9800 \\
\hline $\mathrm{C}\left(55^{\prime}\right)-\mathrm{C}\left(58^{\prime}\right)$ & $1.534(7)$ & $\mathrm{C}(66)-\mathrm{H}(66 \mathrm{C})$ & 0.9800 \\
\hline $\mathrm{C}\left(55^{\prime}\right)-\mathrm{C}\left(57^{\prime}\right)$ & $1.535(7)$ & $\mathrm{P}(1)-\mathrm{Fe}(1)-\mathrm{P}(2)$ & $84.74(3)$ \\
\hline $\mathrm{C}\left(56^{\prime}\right)-\mathrm{H}(56 \mathrm{D})$ & 0.9800 & $\mathrm{P}(1)-\mathrm{Fe}(1)-\mathrm{Br}(1)$ & $118.65(3)$ \\
\hline $\mathrm{C}\left(56^{\prime}\right)-\mathrm{H}(56 \mathrm{E})$ & 0.9800 & $\mathrm{P}(2)-\mathrm{Fe}(1)-\mathrm{Br}(1)$ & $118.27(3)$ \\
\hline $\mathrm{C}\left(56^{\prime}\right)-\mathrm{H}(56 \mathrm{~F})$ & 0.9800 & $\mathrm{P}(1)-\mathrm{Fe}(1)-\mathrm{Br}(2)$ & $109.45(3)$ \\
\hline $\mathrm{C}\left(57^{\prime}\right)-\mathrm{H}(57 \mathrm{D})$ & 0.9800 & $\mathrm{P}(2)-\mathrm{Fe}(1)-\mathrm{Br}(2)$ & $117.81(3)$ \\
\hline $\mathrm{C}\left(57^{\prime}\right)-\mathrm{H}(57 \mathrm{E})$ & 0.9800 & $\operatorname{Br}(1)-\mathrm{Fe}(1)-\mathrm{Br}(2)$ & $106.83(2)$ \\
\hline $\mathrm{C}\left(57^{\prime}\right)-\mathrm{H}(57 \mathrm{~F})$ & 0.9800 & $\mathrm{P}(1)-\mathrm{Fe}(1)-\mathrm{Fe}(1) \# 1$ & $132.98(3)$ \\
\hline $\mathrm{C}\left(58^{\prime}\right)-\mathrm{H}(58 \mathrm{D})$ & 0.9800 & $\mathrm{P}(2)-\mathrm{Fe}(1)-\mathrm{Fe}(1) \# 1$ & $142.07(4)$ \\
\hline $\mathrm{C}\left(58^{\prime}\right)-\mathrm{H}(58 \mathrm{E})$ & 0.9800 & $\operatorname{Br}(1)-\mathrm{Fe}(1)-\mathrm{Fe}(1) \# 1$ & $53.323(14)$ \\
\hline $\mathrm{C}\left(58^{\prime}\right)-\mathrm{H}(58 \mathrm{~F})$ & 0.9800 & $\operatorname{Br}(2)-\mathrm{Fe}(1)-\mathrm{Fe}(1) \# 1$ & $53.510(14)$ \\
\hline $\mathrm{C}(59)-\mathrm{C}(62)$ & $1.528(5)$ & $\mathrm{Fe}(1) \# 1-\mathrm{Br}(1)-\mathrm{Fe}(1)$ & $73.35(3)$ \\
\hline $\mathrm{C}(59)-\mathrm{C}(60)$ & $1.535(5)$ & $\mathrm{Fe}(1) \# 1-\mathrm{Br}(2)-\mathrm{Fe}(1)$ & $72.98(3)$ \\
\hline$C(59)-C(61)$ & $1.535(5)$ & $C(1)-P(1)-C(13)$ & $105.24(15)$ \\
\hline $\mathrm{C}(60)-\mathrm{H}(60 \mathrm{~A})$ & 0.9800 & $\mathrm{C}(1)-\mathrm{P}(1)-\mathrm{C}(7)$ & $100.81(15)$ \\
\hline $\mathrm{C}(60)-\mathrm{H}(60 \mathrm{~B})$ & 0.9800 & $\mathrm{C}(13)-\mathrm{P}(1)-\mathrm{C}(7)$ & $105.44(15)$ \\
\hline $\mathrm{C}(60)-\mathrm{H}(60 \mathrm{C})$ & 0.9800 & $\mathrm{C}(1)-\mathrm{P}(1)-\mathrm{Fe}(1)$ & $109.46(11)$ \\
\hline $\mathrm{C}(61)-\mathrm{H}(61 \mathrm{~A})$ & 0.9800 & $\mathrm{C}(13)-\mathrm{P}(1)-\mathrm{Fe}(1)$ & $116.70(11)$ \\
\hline $\mathrm{C}(61)-\mathrm{H}(61 \mathrm{~B})$ & 0.9800 & $\mathrm{C}(7)-\mathrm{P}(1)-\mathrm{Fe}(1)$ & $117.43(11)$ \\
\hline $\mathrm{C}(61)-\mathrm{H}(61 \mathrm{C})$ & 0.9800 & $C(25)-P(2)-C(19)$ & $101.86(15)$ \\
\hline $\mathrm{C}(62)-\mathrm{H}(62 \mathrm{~A})$ & 0.9800 & $\mathrm{C}(25)-\mathrm{P}(2)-\mathrm{C}(6)$ & $101.13(15)$ \\
\hline $\mathrm{C}(62)-\mathrm{H}(62 \mathrm{~B})$ & 0.9800 & $\mathrm{C}(19)-\mathrm{P}(2)-\mathrm{C}(6)$ & $106.00(15)$ \\
\hline $\mathrm{C}(62)-\mathrm{H}(62 \mathrm{C})$ & 0.9800 & $\mathrm{C}(25)-\mathrm{P}(2)-\mathrm{Fe}(1)$ & $121.84(11)$ \\
\hline
\end{tabular}




\begin{tabular}{|c|c|c|c|}
\hline $\mathrm{C}(19)-\mathrm{P}(2)-\mathrm{Fe}(1)$ & $114.85(11)$ & $\mathrm{C}(7)-\mathrm{C}(12)-\mathrm{H}(12 \mathrm{~A})$ & 119.4 \\
\hline $\mathrm{C}(6)-\mathrm{P}(2)-\mathrm{Fe}(1)$ & $109.38(11)$ & $\mathrm{C}(11)-\mathrm{C}(12)-\mathrm{H}(12 \mathrm{~A})$ & 119.4 \\
\hline$C(2)-C(1)-C(6)$ & $119.3(3)$ & $C(14)-C(13)-C(18)$ & $119.3(3)$ \\
\hline $\mathrm{C}(2)-\mathrm{C}(1)-\mathrm{P}(1)$ & $123.5(3)$ & $C(14)-C(13)-P(1)$ & $119.1(3)$ \\
\hline $\mathrm{C}(6)-\mathrm{C}(1)-\mathrm{P}(1)$ & $117.2(2)$ & $\mathrm{C}(18)-\mathrm{C}(13)-\mathrm{P}(1)$ & $120.7(3)$ \\
\hline$C(3)-C(2)-C(1)$ & $120.7(3)$ & $C(13)-C(14)-C(15)$ & $121.4(3)$ \\
\hline $\mathrm{C}(3)-\mathrm{C}(2)-\mathrm{H}(2 \mathrm{~A})$ & 119.6 & $\mathrm{C}(13)-\mathrm{C}(14)-\mathrm{H}(14 \mathrm{~A})$ & 119.3 \\
\hline $\mathrm{C}(1)-\mathrm{C}(2)-\mathrm{H}(2 \mathrm{~A})$ & 119.6 & $\mathrm{C}(15)-\mathrm{C}(14)-\mathrm{H}(14 \mathrm{~A})$ & 119.3 \\
\hline $\mathrm{C}(4)-\mathrm{C}(3)-\mathrm{C}(2)$ & $119.8(3)$ & $C(16)-C(15)-C(14)$ & $117.4(3)$ \\
\hline $\mathrm{C}(4)-\mathrm{C}(3)-\mathrm{H}(3 \mathrm{~A})$ & 120.1 & $\mathrm{C}(16)-\mathrm{C}(15)-\mathrm{C}(39)$ & $123.1(3)$ \\
\hline $\mathrm{C}(2)-\mathrm{C}(3)-\mathrm{H}(3 \mathrm{~A})$ & 120.1 & $\mathrm{C}(14)-\mathrm{C}(15)-\mathrm{C}(39)$ & $119.5(3)$ \\
\hline $\mathrm{C}(5)-\mathrm{C}(4)-\mathrm{C}(3)$ & $120.0(3)$ & $C(15)-C(16)-C(17)$ & $123.0(3)$ \\
\hline $\mathrm{C}(5)-\mathrm{C}(4)-\mathrm{H}(4 \mathrm{~A})$ & 120.0 & $\mathrm{C}(15)-\mathrm{C}(16)-\mathrm{H}(16 \mathrm{~A})$ & 118.5 \\
\hline $\mathrm{C}(3)-\mathrm{C}(4)-\mathrm{H}(4 \mathrm{~A})$ & 120.0 & $\mathrm{C}(17)-\mathrm{C}(16)-\mathrm{H}(16 \mathrm{~A})$ & 118.5 \\
\hline$C(4)-C(5)-C(6)$ & 121.1(3) & $C(18)-C(17)-C(16)$ & $117.8(3)$ \\
\hline $\mathrm{C}(4)-\mathrm{C}(5)-\mathrm{H}(5 \mathrm{~A})$ & 119.5 & $\mathrm{C}(18)-\mathrm{C}(17)-\mathrm{C}(43)$ & $122.9(3)$ \\
\hline $\mathrm{C}(6)-\mathrm{C}(5)-\mathrm{H}(5 \mathrm{~A})$ & 119.5 & $C(16)-C(17)-C(43)$ & $119.3(3)$ \\
\hline$C(5)-C(6)-C(1)$ & 119.1(3) & $C(17)-C(18)-C(13)$ & $121.0(3)$ \\
\hline$C(5)-C(6)-P(2)$ & $124.4(3)$ & $\mathrm{C}(17)-\mathrm{C}(18)-\mathrm{H}(18 \mathrm{~A})$ & 119.5 \\
\hline$C(1)-C(6)-P(2)$ & $116.4(2)$ & $\mathrm{C}(13)-\mathrm{C}(18)-\mathrm{H}(18 \mathrm{~A})$ & 119.5 \\
\hline $\mathrm{C}(12)-\mathrm{C}(7)-\mathrm{C}(8)$ & 119.7(3) & $C(20)-C(19)-C(24)$ & $118.8(3)$ \\
\hline$C(12)-C(7)-P(1)$ & $125.4(3)$ & $\mathrm{C}(20)-\mathrm{C}(19)-\mathrm{P}(2)$ & $125.9(3)$ \\
\hline $\mathrm{C}(8)-\mathrm{C}(7)-\mathrm{P}(1)$ & $115.0(2)$ & C(24)-C(19)-P(2) & $115.3(3)$ \\
\hline $\mathrm{C}(9)-\mathrm{C}(8)-\mathrm{C}(7)$ & $121.5(3)$ & $C(19)-C(20)-C(21)$ & $121.4(3)$ \\
\hline $\mathrm{C}(9)-\mathrm{C}(8)-\mathrm{H}(8 \mathrm{~A})$ & 119.2 & $\mathrm{C}(19)-\mathrm{C}(20)-\mathrm{H}(20 \mathrm{~A})$ & 119.3 \\
\hline $\mathrm{C}(7)-\mathrm{C}(8)-\mathrm{H}(8 \mathrm{~A})$ & 119.2 & $\mathrm{C}(21)-\mathrm{C}(20)-\mathrm{H}(20 \mathrm{~A})$ & 119.3 \\
\hline $\mathrm{C}(8)-\mathrm{C}(9)-\mathrm{C}(10)$ & 117.1(3) & $C(22)-C(21)-C(20)$ & $117.4(3)$ \\
\hline $\mathrm{C}(8)-\mathrm{C}(9)-\mathrm{C}(31)$ & $122.6(3)$ & $\mathrm{C}(22)-\mathrm{C}(21)-\mathrm{C}(51)$ & $121.3(3)$ \\
\hline $\mathrm{C}(10)-\mathrm{C}(9)-\mathrm{C}(31)$ & $120.3(3)$ & $\mathrm{C}(20)-\mathrm{C}(21)-\mathrm{C}(51)$ & $121.2(3)$ \\
\hline $\mathrm{C}(11)-\mathrm{C}(10)-\mathrm{C}(9)$ & $122.9(3)$ & $C(21)-C(22)-C(23)$ & $123.2(3)$ \\
\hline $\mathrm{C}(11)-\mathrm{C}(10)-\mathrm{H}(10 \mathrm{~A})$ & 118.6 & $\mathrm{C}(21)-\mathrm{C}(22)-\mathrm{H}(22 \mathrm{~A})$ & 118.4 \\
\hline $\mathrm{C}(9)-\mathrm{C}(10)-\mathrm{H}(10 \mathrm{~A})$ & 118.6 & $\mathrm{C}(23)-\mathrm{C}(22)-\mathrm{H}(22 \mathrm{~A})$ & 118.4 \\
\hline$C(10)-C(11)-C(12)$ & $117.7(3)$ & $\mathrm{C}(24)-\mathrm{C}(23)-\mathrm{C}(22)$ & $116.9(3)$ \\
\hline $\mathrm{C}(10)-\mathrm{C}(11)-\mathrm{C}(35)$ & $122.2(3)$ & $\mathrm{C}(24)-\mathrm{C}(23)-\mathrm{C}(47)$ & $122.6(3)$ \\
\hline$C(12)-C(11)-C(35)$ & $120.1(3)$ & $C(22)-C(23)-C(47)$ & $120.5(3)$ \\
\hline$C(7)-C(12)-C(11)$ & 121.1(3) & $C(23)-C(24)-C(19)$ & $122.3(3)$ \\
\hline
\end{tabular}




\begin{tabular}{|c|c|}
\hline $\mathrm{C}(23)-\mathrm{C}(24)-\mathrm{H}(24 \mathrm{~A})$ & 118.9 \\
\hline $\mathrm{C}(19)-\mathrm{C}(24)-\mathrm{H}(24 \mathrm{~A})$ & 118.9 \\
\hline$C(26)-C(25)-C(30)$ & $119.2(3)$ \\
\hline $\mathrm{C}(26)-\mathrm{C}(25)-\mathrm{P}(2)$ & $123.2(3)$ \\
\hline $\mathrm{C}(30)-\mathrm{C}(25)-\mathrm{P}(2)$ & $117.6(2)$ \\
\hline$C(25)-C(26)-C(27)$ & $121.2(3)$ \\
\hline $\mathrm{C}(25)-\mathrm{C}(26)-\mathrm{H}(26 \mathrm{~A})$ & 119.4 \\
\hline $\mathrm{C}(27)-\mathrm{C}(26)-\mathrm{H}(26 \mathrm{~A})$ & 119.4 \\
\hline $\mathrm{C}(28)-\mathrm{C}(27)-\mathrm{C}(26)$ & $117.5(3)$ \\
\hline $\mathrm{C}(28)-\mathrm{C}(27)-\mathrm{C}(55)$ & $120.0(4)$ \\
\hline$C(26)-C(27)-C(55)$ & $122.4(4)$ \\
\hline $\mathrm{C}(28)-\mathrm{C}(27)-\mathrm{C}\left(55^{\prime}\right)$ & $123.2(5)$ \\
\hline$C(26)-C(27)-C\left(55^{\prime}\right)$ & $119.1(5)$ \\
\hline $\mathrm{C}(27)-\mathrm{C}(28)-\mathrm{C}(29)$ & $123.2(3)$ \\
\hline $\mathrm{C}(27)-\mathrm{C}(28)-\mathrm{H}(28 \mathrm{~A})$ & 118.4 \\
\hline $\mathrm{C}(29)-\mathrm{C}(28)-\mathrm{H}(28 \mathrm{~A})$ & 118.4 \\
\hline $\mathrm{C}(30)-\mathrm{C}(29)-\mathrm{C}(28)$ & $117.3(3)$ \\
\hline $\mathrm{C}(30)-\mathrm{C}(29)-\mathrm{C}(59)$ & $123.3(3)$ \\
\hline$C(28)-C(29)-C(59)$ & $119.4(3)$ \\
\hline$C(29)-C(30)-C(25)$ & $121.5(3)$ \\
\hline $\mathrm{C}(29)-\mathrm{C}(30)-\mathrm{H}(30 \mathrm{~A})$ & 119.2 \\
\hline $\mathrm{C}(25)-\mathrm{C}(30)-\mathrm{H}(30 \mathrm{~A})$ & 119.2 \\
\hline $\mathrm{C}(33)-\mathrm{C}(31)-\mathrm{C}(34)$ & $108.8(3)$ \\
\hline$C(33)-C(31)-C(9)$ & $110.0(3)$ \\
\hline$C(34)-C(31)-C(9)$ & $112.3(3)$ \\
\hline$C(33)-C(31)-C(32)$ & $109.9(3)$ \\
\hline $\mathrm{C}(34)-\mathrm{C}(31)-\mathrm{C}(32)$ & $107.5(3)$ \\
\hline$C(9)-C(31)-C(32)$ & $108.5(3)$ \\
\hline $\mathrm{C}(31)-\mathrm{C}(32)-\mathrm{H}(32 \mathrm{~A})$ & 109.5 \\
\hline $\mathrm{C}(31)-\mathrm{C}(32)-\mathrm{H}(32 \mathrm{~B})$ & 109.5 \\
\hline $\mathrm{H}(32 \mathrm{~A})-\mathrm{C}(32)-\mathrm{H}(32 \mathrm{~B})$ & 109.5 \\
\hline $\mathrm{C}(31)-\mathrm{C}(32)-\mathrm{H}(32 \mathrm{C})$ & 109.5 \\
\hline $\mathrm{H}(32 \mathrm{~A})-\mathrm{C}(32)-\mathrm{H}(32 \mathrm{C})$ & 109.5 \\
\hline $\mathrm{H}(32 \mathrm{~B})-\mathrm{C}(32)-\mathrm{H}(32 \mathrm{C})$ & 109.5 \\
\hline $\mathrm{C}(31)-\mathrm{C}(33)-\mathrm{H}(33 \mathrm{~A})$ & 109.5 \\
\hline $\mathrm{C}(31)-\mathrm{C}(33)-\mathrm{H}(33 \mathrm{~B})$ & 109.5 \\
\hline
\end{tabular}

\begin{tabular}{|c|c|}
\hline $\mathrm{H}(33 \mathrm{~A})-\mathrm{C}(33)-\mathrm{H}(33 \mathrm{~B})$ & 109.5 \\
\hline $\mathrm{C}(31)-\mathrm{C}(33)-\mathrm{H}(33 \mathrm{C})$ & 109.5 \\
\hline $\mathrm{H}(33 \mathrm{~A})-\mathrm{C}(33)-\mathrm{H}(33 \mathrm{C})$ & 109.5 \\
\hline $\mathrm{H}(33 \mathrm{~B})-\mathrm{C}(33)-\mathrm{H}(33 \mathrm{C})$ & 109.5 \\
\hline $\mathrm{C}(31)-\mathrm{C}(34)-\mathrm{H}(34 \mathrm{~A})$ & 109.5 \\
\hline $\mathrm{C}(31)-\mathrm{C}(34)-\mathrm{H}(34 \mathrm{~B})$ & 109.5 \\
\hline $\mathrm{H}(34 \mathrm{~A})-\mathrm{C}(34)-\mathrm{H}(34 \mathrm{~B})$ & 109.5 \\
\hline $\mathrm{C}(31)-\mathrm{C}(34)-\mathrm{H}(34 \mathrm{C})$ & 109.5 \\
\hline $\mathrm{H}(34 \mathrm{~A})-\mathrm{C}(34)-\mathrm{H}(34 \mathrm{C})$ & 109.5 \\
\hline $\mathrm{H}(34 \mathrm{~B})-\mathrm{C}(34)-\mathrm{H}(34 \mathrm{C})$ & 109.5 \\
\hline $\mathrm{C}(37)-\mathrm{C}(35)-\mathrm{C}(38)$ & $108.3(3)$ \\
\hline $\mathrm{C}(37)-\mathrm{C}(35)-\mathrm{C}(11)$ & $112.6(3)$ \\
\hline $\mathrm{C}(38)-\mathrm{C}(35)-\mathrm{C}(11)$ & 109.1(3) \\
\hline $\mathrm{C}(37)-\mathrm{C}(35)-\mathrm{C}(36)$ & $108.7(3)$ \\
\hline $\mathrm{C}(38)-\mathrm{C}(35)-\mathrm{C}(36)$ & $109.1(3)$ \\
\hline $\mathrm{C}(11)-\mathrm{C}(35)-\mathrm{C}(36)$ & $109.0(3)$ \\
\hline $\mathrm{C}(35)-\mathrm{C}(36)-\mathrm{H}(36 \mathrm{~A})$ & 109.5 \\
\hline $\mathrm{C}(35)-\mathrm{C}(36)-\mathrm{H}(36 \mathrm{~B})$ & 109.5 \\
\hline $\mathrm{H}(36 \mathrm{~A})-\mathrm{C}(36)-\mathrm{H}(36 \mathrm{~B})$ & 109.5 \\
\hline $\mathrm{C}(35)-\mathrm{C}(36)-\mathrm{H}(36 \mathrm{C})$ & 109.5 \\
\hline $\mathrm{H}(36 \mathrm{~A})-\mathrm{C}(36)-\mathrm{H}(36 \mathrm{C})$ & 109.5 \\
\hline $\mathrm{H}(36 \mathrm{~B})-\mathrm{C}(36)-\mathrm{H}(36 \mathrm{C})$ & 109.5 \\
\hline $\mathrm{C}(35)-\mathrm{C}(37)-\mathrm{H}(37 \mathrm{~A})$ & 109.5 \\
\hline $\mathrm{C}(35)-\mathrm{C}(37)-\mathrm{H}(37 \mathrm{~B})$ & 109.5 \\
\hline $\mathrm{H}(37 \mathrm{~A})-\mathrm{C}(37)-\mathrm{H}(37 \mathrm{~B})$ & 109.5 \\
\hline $\mathrm{C}(35)-\mathrm{C}(37)-\mathrm{H}(37 \mathrm{C})$ & 109.5 \\
\hline $\mathrm{H}(37 \mathrm{~A})-\mathrm{C}(37)-\mathrm{H}(37 \mathrm{C})$ & 109.5 \\
\hline $\mathrm{H}(37 \mathrm{~B})-\mathrm{C}(37)-\mathrm{H}(37 \mathrm{C})$ & 109.5 \\
\hline $\mathrm{C}(35)-\mathrm{C}(38)-\mathrm{H}(38 \mathrm{~A})$ & 109.5 \\
\hline $\mathrm{C}(35)-\mathrm{C}(38)-\mathrm{H}(38 \mathrm{~B})$ & 109.5 \\
\hline $\mathrm{H}(38 \mathrm{~A})-\mathrm{C}(38)-\mathrm{H}(38 \mathrm{~B})$ & 109.5 \\
\hline $\mathrm{C}(35)-\mathrm{C}(38)-\mathrm{H}(38 \mathrm{C})$ & 109.5 \\
\hline $\mathrm{H}(38 \mathrm{~A})-\mathrm{C}(38)-\mathrm{H}(38 \mathrm{C})$ & 109.5 \\
\hline $\mathrm{H}(38 \mathrm{~B})-\mathrm{C}(38)-\mathrm{H}(38 \mathrm{C})$ & 109.5 \\
\hline $\mathrm{C}(42)-\mathrm{C}(39)-\mathrm{C}(40)$ & 109.1(3) \\
\hline C(42)-C(39)-C(41) & $108.6(3)$ \\
\hline
\end{tabular}




\begin{tabular}{|c|c|}
\hline$C(40)-C(39)-C(41)$ & $109.2(3)$ \\
\hline$C(42)-C(39)-C(15)$ & $112.1(3)$ \\
\hline$C(40)-C(39)-C(15)$ & $108.8(3)$ \\
\hline$C(41)-C(39)-C(15)$ & $109.0(3)$ \\
\hline $\mathrm{C}(39)-\mathrm{C}(40)-\mathrm{H}(40 \mathrm{~A})$ & 109.5 \\
\hline $\mathrm{C}(39)-\mathrm{C}(40)-\mathrm{H}(40 \mathrm{~B})$ & 109.5 \\
\hline $\mathrm{H}(40 \mathrm{~A})-\mathrm{C}(40)-\mathrm{H}(40 \mathrm{~B})$ & 109.5 \\
\hline $\mathrm{C}(39)-\mathrm{C}(40)-\mathrm{H}(40 \mathrm{C})$ & 109.5 \\
\hline $\mathrm{H}(40 \mathrm{~A})-\mathrm{C}(40)-\mathrm{H}(40 \mathrm{C})$ & 109.5 \\
\hline $\mathrm{H}(40 \mathrm{~B})-\mathrm{C}(40)-\mathrm{H}(40 \mathrm{C})$ & 109.5 \\
\hline $\mathrm{C}(39)-\mathrm{C}(41)-\mathrm{H}(41 \mathrm{~A})$ & 109.5 \\
\hline $\mathrm{C}(39)-\mathrm{C}(41)-\mathrm{H}(41 \mathrm{~B})$ & 109.5 \\
\hline $\mathrm{H}(41 \mathrm{~A})-\mathrm{C}(41)-\mathrm{H}(41 \mathrm{~B})$ & 109.5 \\
\hline C(39)-C(41)-H(41C) & 109.5 \\
\hline $\mathrm{H}(41 \mathrm{~A})-\mathrm{C}(41)-\mathrm{H}(41 \mathrm{C})$ & 109.5 \\
\hline $\mathrm{H}(41 \mathrm{~B})-\mathrm{C}(41)-\mathrm{H}(41 \mathrm{C})$ & 109.5 \\
\hline $\mathrm{C}(39)-\mathrm{C}(42)-\mathrm{H}(42 \mathrm{~A})$ & 109.5 \\
\hline $\mathrm{C}(39)-\mathrm{C}(42)-\mathrm{H}(42 \mathrm{~B})$ & 109.5 \\
\hline $\mathrm{H}(42 \mathrm{~A})-\mathrm{C}(42)-\mathrm{H}(42 \mathrm{~B})$ & 109.5 \\
\hline $\mathrm{C}(39)-\mathrm{C}(42)-\mathrm{H}(42 \mathrm{C})$ & 109.5 \\
\hline $\mathrm{H}(42 \mathrm{~A})-\mathrm{C}(42)-\mathrm{H}(42 \mathrm{C})$ & 109.5 \\
\hline $\mathrm{H}(42 \mathrm{~B})-\mathrm{C}(42)-\mathrm{H}(42 \mathrm{C})$ & 109.5 \\
\hline$C(45)-C(43)-C(17)$ & $111.6(3)$ \\
\hline$C(45)-C(43)-C(44)$ & $108.7(3)$ \\
\hline$C(17)-C(43)-C(44)$ & $109.0(3)$ \\
\hline$C(45)-C(43)-C(46)$ & $108.8(3)$ \\
\hline$C(17)-C(43)-C(46)$ & 109.1(3) \\
\hline $\mathrm{C}(44)-\mathrm{C}(43)-\mathrm{C}(46)$ & $109.6(3)$ \\
\hline $\mathrm{C}(43)-\mathrm{C}(44)-\mathrm{H}(44 \mathrm{~A})$ & 109.5 \\
\hline $\mathrm{C}(43)-\mathrm{C}(44)-\mathrm{H}(44 \mathrm{~B})$ & 109.5 \\
\hline $\mathrm{H}(44 \mathrm{~A})-\mathrm{C}(44)-\mathrm{H}(44 \mathrm{~B})$ & 109.5 \\
\hline $\mathrm{C}(43)-\mathrm{C}(44)-\mathrm{H}(44 \mathrm{C})$ & 109.5 \\
\hline $\mathrm{H}(44 \mathrm{~A})-\mathrm{C}(44)-\mathrm{H}(44 \mathrm{C})$ & 109.5 \\
\hline $\mathrm{H}(44 \mathrm{~B})-\mathrm{C}(44)-\mathrm{H}(44 \mathrm{C})$ & 109.5 \\
\hline $\mathrm{C}(43)-\mathrm{C}(45)-\mathrm{H}(45 \mathrm{~A})$ & 109.5 \\
\hline $\mathrm{C}(43)-\mathrm{C}(45)-\mathrm{H}(45 \mathrm{~B})$ & 109.5 \\
\hline
\end{tabular}

\begin{tabular}{|c|c|}
\hline $\mathrm{H}(45 \mathrm{~A})-\mathrm{C}(45)-\mathrm{H}(45 \mathrm{~B})$ & 109.5 \\
\hline $\mathrm{C}(43)-\mathrm{C}(45)-\mathrm{H}(45 \mathrm{C})$ & 109.5 \\
\hline $\mathrm{H}(45 \mathrm{~A})-\mathrm{C}(45)-\mathrm{H}(45 \mathrm{C})$ & 109.5 \\
\hline $\mathrm{H}(45 \mathrm{~B})-\mathrm{C}(45)-\mathrm{H}(45 \mathrm{C})$ & 109.5 \\
\hline $\mathrm{C}(43)-\mathrm{C}(46)-\mathrm{H}(46 \mathrm{~A})$ & 109.5 \\
\hline $\mathrm{C}(43)-\mathrm{C}(46)-\mathrm{H}(46 \mathrm{~B})$ & 109.5 \\
\hline $\mathrm{H}(46 \mathrm{~A})-\mathrm{C}(46)-\mathrm{H}(46 \mathrm{~B})$ & 109.5 \\
\hline $\mathrm{C}(43)-\mathrm{C}(46)-\mathrm{H}(46 \mathrm{C})$ & 109.5 \\
\hline $\mathrm{H}(46 \mathrm{~A})-\mathrm{C}(46)-\mathrm{H}(46 \mathrm{C})$ & 109.5 \\
\hline $\mathrm{H}(46 \mathrm{~B})-\mathrm{C}(46)-\mathrm{H}(46 \mathrm{C})$ & 109.5 \\
\hline $\mathrm{C}(50)-\mathrm{C}(47)-\mathrm{C}(23)$ & $111.8(3)$ \\
\hline $\mathrm{C}(50)-\mathrm{C}(47)-\mathrm{C}(48)$ & $107.9(3)$ \\
\hline$C(23)-C(47)-C(48)$ & $111.0(3)$ \\
\hline $\mathrm{C}(50)-\mathrm{C}(47)-\mathrm{C}(49)$ & 109.2(3) \\
\hline $\mathrm{C}(23)-\mathrm{C}(47)-\mathrm{C}(49)$ & $107.8(3)$ \\
\hline $\mathrm{C}(48)-\mathrm{C}(47)-\mathrm{C}(49)$ & 109.1(3) \\
\hline $\mathrm{C}(47)-\mathrm{C}(48)-\mathrm{H}(48 \mathrm{~A})$ & 109.5 \\
\hline $\mathrm{C}(47)-\mathrm{C}(48)-\mathrm{H}(48 \mathrm{~B})$ & 109.5 \\
\hline $\mathrm{H}(48 \mathrm{~A})-\mathrm{C}(48)-\mathrm{H}(48 \mathrm{~B})$ & 109.5 \\
\hline $\mathrm{C}(47)-\mathrm{C}(48)-\mathrm{H}(48 \mathrm{C})$ & 109.5 \\
\hline $\mathrm{H}(48 \mathrm{~A})-\mathrm{C}(48)-\mathrm{H}(48 \mathrm{C})$ & 109.5 \\
\hline $\mathrm{H}(48 \mathrm{~B})-\mathrm{C}(48)-\mathrm{H}(48 \mathrm{C})$ & 109.5 \\
\hline $\mathrm{C}(47)-\mathrm{C}(49)-\mathrm{H}(49 \mathrm{~A})$ & 109.5 \\
\hline $\mathrm{C}(47)-\mathrm{C}(49)-\mathrm{H}(49 \mathrm{~B})$ & 109.5 \\
\hline $\mathrm{H}(49 \mathrm{~A})-\mathrm{C}(49)-\mathrm{H}(49 \mathrm{~B})$ & 109.5 \\
\hline $\mathrm{C}(47)-\mathrm{C}(49)-\mathrm{H}(49 \mathrm{C})$ & 109.5 \\
\hline $\mathrm{H}(49 \mathrm{~A})-\mathrm{C}(49)-\mathrm{H}(49 \mathrm{C})$ & 109.5 \\
\hline $\mathrm{H}(49 \mathrm{~B})-\mathrm{C}(49)-\mathrm{H}(49 \mathrm{C})$ & 109.5 \\
\hline $\mathrm{C}(47)-\mathrm{C}(50)-\mathrm{H}(50 \mathrm{~A})$ & 109.5 \\
\hline $\mathrm{C}(47)-\mathrm{C}(50)-\mathrm{H}(50 \mathrm{~B})$ & 109.5 \\
\hline $\mathrm{H}(50 \mathrm{~A})-\mathrm{C}(50)-\mathrm{H}(50 \mathrm{~B})$ & 109.5 \\
\hline $\mathrm{C}(47)-\mathrm{C}(50)-\mathrm{H}(50 \mathrm{C})$ & 109.5 \\
\hline $\mathrm{H}(50 \mathrm{~A})-\mathrm{C}(50)-\mathrm{H}(50 \mathrm{C})$ & 109.5 \\
\hline $\mathrm{H}(50 \mathrm{~B})-\mathrm{C}(50)-\mathrm{H}(50 \mathrm{C})$ & 109.5 \\
\hline$C(52)-C(51)-C(21)$ & $111.0(3)$ \\
\hline $\mathrm{C}(52)-\mathrm{C}(51)-\mathrm{C}(54)$ & $109.6(3)$ \\
\hline
\end{tabular}




\begin{tabular}{|c|c|c|c|}
\hline $\mathrm{C}(21)-\mathrm{C}(51)-\mathrm{C}(54)$ & $108.0(3)$ & $\mathrm{H}(57 \mathrm{~A})-\mathrm{C}(57)-\mathrm{H}(57 \mathrm{~B})$ & 109.5 \\
\hline$C(52)-C(51)-C(53)$ & $107.5(3)$ & $\mathrm{C}(55)-\mathrm{C}(57)-\mathrm{H}(57 \mathrm{C})$ & 109.5 \\
\hline $\mathrm{C}(21)-\mathrm{C}(51)-\mathrm{C}(53)$ & $112.6(3)$ & $\mathrm{H}(57 \mathrm{~A})-\mathrm{C}(57)-\mathrm{H}(57 \mathrm{C})$ & 109.5 \\
\hline$C(54)-C(51)-C(53)$ & $108.1(3)$ & $\mathrm{H}(57 \mathrm{~B})-\mathrm{C}(57)-\mathrm{H}(57 \mathrm{C})$ & 109.5 \\
\hline $\mathrm{C}(51)-\mathrm{C}(52)-\mathrm{H}(52 \mathrm{~A})$ & 109.5 & $\mathrm{C}(55)-\mathrm{C}(58)-\mathrm{H}(58 \mathrm{~A})$ & 109.5 \\
\hline $\mathrm{C}(51)-\mathrm{C}(52)-\mathrm{H}(52 \mathrm{~B})$ & 109.5 & $\mathrm{C}(55)-\mathrm{C}(58)-\mathrm{H}(58 \mathrm{~B})$ & 109.5 \\
\hline $\mathrm{H}(52 \mathrm{~A})-\mathrm{C}(52)-\mathrm{H}(52 \mathrm{~B})$ & 109.5 & $\mathrm{H}(58 \mathrm{~A})-\mathrm{C}(58)-\mathrm{H}(58 \mathrm{~B})$ & 109.5 \\
\hline $\mathrm{C}(51)-\mathrm{C}(52)-\mathrm{H}(52 \mathrm{C})$ & 109.5 & $\mathrm{C}(55)-\mathrm{C}(58)-\mathrm{H}(58 \mathrm{C})$ & 109.5 \\
\hline $\mathrm{H}(52 \mathrm{~A})-\mathrm{C}(52)-\mathrm{H}(52 \mathrm{C})$ & 109.5 & $\mathrm{H}(58 \mathrm{~A})-\mathrm{C}(58)-\mathrm{H}(58 \mathrm{C})$ & 109.5 \\
\hline $\mathrm{H}(52 \mathrm{~B})-\mathrm{C}(52)-\mathrm{H}(52 \mathrm{C})$ & 109.5 & $\mathrm{H}(58 \mathrm{~B})-\mathrm{C}(58)-\mathrm{H}(58 \mathrm{C})$ & 109.5 \\
\hline $\mathrm{C}(51)-\mathrm{C}(53)-\mathrm{H}(53 \mathrm{~A})$ & 109.5 & $\mathrm{C}\left(56^{\prime}\right)-\mathrm{C}\left(55^{\prime}\right)-\mathrm{C}(27)$ & $111.9(6)$ \\
\hline $\mathrm{C}(51)-\mathrm{C}(53)-\mathrm{H}(53 \mathrm{~B})$ & 109.5 & $\mathrm{C}\left(56^{\prime}\right)-\mathrm{C}\left(55^{\prime}\right)-\mathrm{C}\left(58^{\prime}\right)$ & $108.0(7)$ \\
\hline $\mathrm{H}(53 \mathrm{~A})-\mathrm{C}(53)-\mathrm{H}(53 \mathrm{~B})$ & 109.5 & $\mathrm{C}(27)-\mathrm{C}\left(55^{\prime}\right)-\mathrm{C}\left(58^{\prime}\right)$ & $112.1(7)$ \\
\hline $\mathrm{C}(51)-\mathrm{C}(53)-\mathrm{H}(53 \mathrm{C})$ & 109.5 & $\mathrm{C}\left(56^{\prime}\right)-\mathrm{C}\left(55^{\prime}\right)-\mathrm{C}\left(57^{\prime}\right)$ & $108.8(7)$ \\
\hline $\mathrm{H}(53 \mathrm{~A})-\mathrm{C}(53)-\mathrm{H}(53 \mathrm{C})$ & 109.5 & $\mathrm{C}(27)-\mathrm{C}\left(55^{\prime}\right)-\mathrm{C}\left(57^{\prime}\right)$ & $108.1(7)$ \\
\hline $\mathrm{H}(53 \mathrm{~B})-\mathrm{C}(53)-\mathrm{H}(53 \mathrm{C})$ & 109.5 & $\mathrm{C}\left(58^{\prime}\right)-\mathrm{C}\left(55^{\prime}\right)-\mathrm{C}\left(57^{\prime}\right)$ & $107.7(7)$ \\
\hline $\mathrm{C}(51)-\mathrm{C}(54)-\mathrm{H}(54 \mathrm{~A})$ & 109.5 & $\mathrm{C}\left(55^{\prime}\right)-\mathrm{C}\left(56^{\prime}\right)-\mathrm{H}(56 \mathrm{D})$ & 109.5 \\
\hline $\mathrm{C}(51)-\mathrm{C}(54)-\mathrm{H}(54 \mathrm{~B})$ & 109.5 & $\mathrm{C}\left(55^{\prime}\right)-\mathrm{C}\left(56^{\prime}\right)-\mathrm{H}(56 \mathrm{E})$ & 109.5 \\
\hline $\mathrm{H}(54 \mathrm{~A})-\mathrm{C}(54)-\mathrm{H}(54 \mathrm{~B})$ & 109.5 & $\mathrm{H}(56 \mathrm{D})-\mathrm{C}\left(56^{\prime}\right)-\mathrm{H}(56 \mathrm{E})$ & 109.5 \\
\hline $\mathrm{C}(51)-\mathrm{C}(54)-\mathrm{H}(54 \mathrm{C})$ & 109.5 & $\mathrm{C}\left(55^{\prime}\right)-\mathrm{C}\left(56^{\prime}\right)-\mathrm{H}(56 \mathrm{~F})$ & 109.5 \\
\hline $\mathrm{H}(54 \mathrm{~A})-\mathrm{C}(54)-\mathrm{H}(54 \mathrm{C})$ & 109.5 & $\mathrm{H}(56 \mathrm{D})-\mathrm{C}\left(56^{\prime}\right)-\mathrm{H}(56 \mathrm{~F})$ & 109.5 \\
\hline $\mathrm{H}(54 \mathrm{~B})-\mathrm{C}(54)-\mathrm{H}(54 \mathrm{C})$ & 109.5 & $\mathrm{H}(56 \mathrm{E})-\mathrm{C}\left(56^{\prime}\right)-\mathrm{H}(56 \mathrm{~F})$ & 109.5 \\
\hline$C(57)-C(55)-C(58)$ & $110.1(6)$ & $\mathrm{C}\left(55^{\prime}\right)-\mathrm{C}\left(57^{\prime}\right)-\mathrm{H}(57 \mathrm{D})$ & 109.5 \\
\hline$C(57)-C(55)-C(27)$ & $108.4(5)$ & $\mathrm{C}\left(55^{\prime}\right)-\mathrm{C}\left(57^{\prime}\right)-\mathrm{H}(57 \mathrm{E})$ & 109.5 \\
\hline $\mathrm{C}(58)-\mathrm{C}(55)-\mathrm{C}(27)$ & $110.9(5)$ & $\mathrm{H}(57 \mathrm{D})-\mathrm{C}\left(57^{\prime}\right)-\mathrm{H}(57 \mathrm{E})$ & 109.5 \\
\hline$C(57)-C(55)-C(56)$ & $108.9(5)$ & $\mathrm{C}\left(55^{\prime}\right)-\mathrm{C}\left(57^{\prime}\right)-\mathrm{H}(57 \mathrm{~F})$ & 109.5 \\
\hline$C(58)-C(55)-C(56)$ & $107.9(6)$ & $\mathrm{H}(57 \mathrm{D})-\mathrm{C}\left(57^{\prime}\right)-\mathrm{H}(57 \mathrm{~F})$ & 109.5 \\
\hline $\mathrm{C}(27)-\mathrm{C}(55)-\mathrm{C}(56)$ & $110.6(5)$ & $\mathrm{H}(57 \mathrm{E})-\mathrm{C}\left(57^{\prime}\right)-\mathrm{H}(57 \mathrm{~F})$ & 109.5 \\
\hline $\mathrm{C}(55)-\mathrm{C}(56)-\mathrm{H}(56 \mathrm{~A})$ & 109.5 & $\mathrm{C}\left(55^{\prime}\right)-\mathrm{C}\left(58^{\prime}\right)-\mathrm{H}(58 \mathrm{D})$ & 109.5 \\
\hline $\mathrm{C}(55)-\mathrm{C}(56)-\mathrm{H}(56 \mathrm{~B})$ & 109.5 & $\mathrm{C}\left(55^{\prime}\right)-\mathrm{C}\left(58^{\prime}\right)-\mathrm{H}(58 \mathrm{E})$ & 109.5 \\
\hline $\mathrm{H}(56 \mathrm{~A})-\mathrm{C}(56)-\mathrm{H}(56 \mathrm{~B})$ & 109.5 & $\mathrm{H}(58 \mathrm{D})-\mathrm{C}\left(58^{\prime}\right)-\mathrm{H}(58 \mathrm{E})$ & 109.5 \\
\hline $\mathrm{C}(55)-\mathrm{C}(56)-\mathrm{H}(56 \mathrm{C})$ & 109.5 & $\mathrm{C}\left(55^{\prime}\right)-\mathrm{C}\left(58^{\prime}\right)-\mathrm{H}(58 \mathrm{~F})$ & 109.5 \\
\hline $\mathrm{H}(56 \mathrm{~A})-\mathrm{C}(56)-\mathrm{H}(56 \mathrm{C})$ & 109.5 & $\mathrm{H}(58 \mathrm{D})-\mathrm{C}\left(58^{\prime}\right)-\mathrm{H}(58 \mathrm{~F})$ & 109.5 \\
\hline $\mathrm{H}(56 \mathrm{~B})-\mathrm{C}(56)-\mathrm{H}(56 \mathrm{C})$ & 109.5 & $\mathrm{H}(58 \mathrm{E})-\mathrm{C}\left(58^{\prime}\right)-\mathrm{H}(58 \mathrm{~F})$ & 109.5 \\
\hline $\mathrm{C}(55)-\mathrm{C}(57)-\mathrm{H}(57 \mathrm{~A})$ & 109.5 & $\mathrm{C}(29)-\mathrm{C}(59)-\mathrm{C}(62)$ & $109.7(3)$ \\
\hline $\mathrm{C}(55)-\mathrm{C}(57)-\mathrm{H}(57 \mathrm{~B})$ & 109.5 & $\mathrm{C}(29)-\mathrm{C}(59)-\mathrm{C}(60)$ & $112.3(3)$ \\
\hline
\end{tabular}




\begin{tabular}{|c|c|c|c|}
\hline $\mathrm{C}(62)-\mathrm{C}(59)-\mathrm{C}(60)$ & $107.9(3)$ & $\mathrm{H}(63 \mathrm{~A})-\mathrm{C}(63)-\mathrm{H}(63 \mathrm{~B})$ & 109.5 \\
\hline $\mathrm{C}(29)-\mathrm{C}(59)-\mathrm{C}(61)$ & $109.5(3)$ & $\mathrm{C}(64)-\mathrm{C}(63)-\mathrm{H}(63 \mathrm{C})$ & 109.5 \\
\hline$C(62)-C(59)-C(61)$ & 109.1(3) & $\mathrm{H}(63 \mathrm{~A})-\mathrm{C}(63)-\mathrm{H}(63 \mathrm{C})$ & 109.5 \\
\hline $\mathrm{C}(60)-\mathrm{C}(59)-\mathrm{C}(61)$ & $108.3(3)$ & $\mathrm{H}(63 \mathrm{~B})-\mathrm{C}(63)-\mathrm{H}(63 \mathrm{C})$ & 109.5 \\
\hline $\mathrm{C}(59)-\mathrm{C}(60)-\mathrm{H}(60 \mathrm{~A})$ & 109.5 & $\mathrm{O}(1)-\mathrm{C}(64)-\mathrm{C}(63)$ & $105.6(16)$ \\
\hline $\mathrm{C}(59)-\mathrm{C}(60)-\mathrm{H}(60 \mathrm{~B})$ & 109.5 & $\mathrm{O}(1)-\mathrm{C}(64)-\mathrm{H}(64 \mathrm{~A})$ & 110.6 \\
\hline $\mathrm{H}(60 \mathrm{~A})-\mathrm{C}(60)-\mathrm{H}(60 \mathrm{~B})$ & 109.5 & $\mathrm{C}(63)-\mathrm{C}(64)-\mathrm{H}(64 \mathrm{~A})$ & 110.6 \\
\hline$C(59)-C(60)-H(60 C)$ & 109.5 & $\mathrm{O}(1)-\mathrm{C}(64)-\mathrm{H}(64 \mathrm{~B})$ & 110.6 \\
\hline $\mathrm{H}(60 \mathrm{~A})-\mathrm{C}(60)-\mathrm{H}(60 \mathrm{C})$ & 109.5 & $\mathrm{C}(63)-\mathrm{C}(64)-\mathrm{H}(64 \mathrm{~B})$ & 110.6 \\
\hline $\mathrm{H}(60 \mathrm{~B})-\mathrm{C}(60)-\mathrm{H}(60 \mathrm{C})$ & 109.5 & $\mathrm{H}(64 \mathrm{~A})-\mathrm{C}(64)-\mathrm{H}(64 \mathrm{~B})$ & 108.8 \\
\hline $\mathrm{C}(59)-\mathrm{C}(61)-\mathrm{H}(61 \mathrm{~A})$ & 109.5 & $\mathrm{C}(64)-\mathrm{O}(1)-\mathrm{C}(65)$ & $108(2)$ \\
\hline $\mathrm{C}(59)-\mathrm{C}(61)-\mathrm{H}(61 \mathrm{~B})$ & 109.5 & $\mathrm{O}(1)-\mathrm{C}(65)-\mathrm{C}(66)$ & $103.5(14)$ \\
\hline $\mathrm{H}(61 \mathrm{~A})-\mathrm{C}(61)-\mathrm{H}(61 \mathrm{~B})$ & 109.5 & $\mathrm{O}(1)-\mathrm{C}(65)-\mathrm{H}(65 \mathrm{~A})$ & 111.1 \\
\hline $\mathrm{C}(59)-\mathrm{C}(61)-\mathrm{H}(61 \mathrm{C})$ & 109.5 & $C(66)-C(65)-H(65 A)$ & 111.1 \\
\hline $\mathrm{H}(61 \mathrm{~A})-\mathrm{C}(61)-\mathrm{H}(61 \mathrm{C})$ & 109.5 & $\mathrm{O}(1)-\mathrm{C}(65)-\mathrm{H}(65 \mathrm{~B})$ & 111.1 \\
\hline $\mathrm{H}(61 \mathrm{~B})-\mathrm{C}(61)-\mathrm{H}(61 \mathrm{C})$ & 109.5 & $\mathrm{C}(66)-\mathrm{C}(65)-\mathrm{H}(65 \mathrm{~B})$ & 111.1 \\
\hline $\mathrm{C}(59)-\mathrm{C}(62)-\mathrm{H}(62 \mathrm{~A})$ & 109.5 & $\mathrm{H}(65 \mathrm{~A})-\mathrm{C}(65)-\mathrm{H}(65 \mathrm{~B})$ & 109.0 \\
\hline $\mathrm{C}(59)-\mathrm{C}(62)-\mathrm{H}(62 \mathrm{~B})$ & 109.5 & $\mathrm{C}(65)-\mathrm{C}(66)-\mathrm{H}(66 \mathrm{~A})$ & 109.5 \\
\hline $\mathrm{H}(62 \mathrm{~A})-\mathrm{C}(62)-\mathrm{H}(62 \mathrm{~B})$ & 109.5 & $\mathrm{C}(65)-\mathrm{C}(66)-\mathrm{H}(66 \mathrm{~B})$ & 109.5 \\
\hline $\mathrm{C}(59)-\mathrm{C}(62)-\mathrm{H}(62 \mathrm{C})$ & 109.5 & $\mathrm{H}(66 \mathrm{~A})-\mathrm{C}(66)-\mathrm{H}(66 \mathrm{~B})$ & 109.5 \\
\hline $\mathrm{H}(62 \mathrm{~A})-\mathrm{C}(62)-\mathrm{H}(62 \mathrm{C})$ & 109.5 & $\mathrm{C}(65)-\mathrm{C}(66)-\mathrm{H}(66 \mathrm{C})$ & 109.5 \\
\hline $\mathrm{H}(62 \mathrm{~B})-\mathrm{C}(62)-\mathrm{H}(62 \mathrm{C})$ & 109.5 & $\mathrm{H}(66 \mathrm{~A})-\mathrm{C}(66)-\mathrm{H}(66 \mathrm{C})$ & 109.5 \\
\hline $\mathrm{C}(64)-\mathrm{C}(63)-\mathrm{H}(63 \mathrm{~A})$ & 109.5 & $\mathrm{H}(66 \mathrm{~B})-\mathrm{C}(66)-\mathrm{H}(66 \mathrm{C})$ & 109.5 \\
\hline $\mathrm{C}(64)-\mathrm{C}(63)-\mathrm{H}(63 \mathrm{~B})$ & 109.5 & & \\
\hline
\end{tabular}

Symmetry transformations used to generate equivalent atoms:

$\# 1-\mathrm{x}+1 / 2, \mathrm{y},-\mathrm{z}+1 / 2$ 
Table S28. Anisotropic displacement parameters $\left(\AA^{2} \times 10^{3}\right)$ for neijk39. The anisotropic displacement factor exponent takes the form: $-2 \pi^{2}\left[h^{2} a^{* 2} U_{11}+\ldots+2 h k a^{*} b^{*} U_{12}\right]$

\begin{tabular}{|c|c|c|c|c|c|c|}
\hline & $\mathrm{U}_{11}$ & $\mathrm{U}_{22}$ & $\mathrm{U}_{33}$ & $\mathrm{U}_{23}$ & $\mathrm{U}_{13}$ & $\mathrm{U}_{12}$ \\
\hline $\mathrm{Fe} 1$ & $18(1)$ & $18(1)$ & $15(1)$ & $1(1)$ & $3(1)$ & $1(1)$ \\
\hline $\mathrm{Br} 1$ & $40(1)$ & $18(1)$ & $28(1)$ & 0 & $18(1)$ & 0 \\
\hline $\mathrm{Br} 2$ & $22(1)$ & $18(1)$ & $20(1)$ & 0 & $7(1)$ & 0 \\
\hline $\mathrm{P} 1$ & 19(1) & $17(1)$ & $14(1)$ & $0(1)$ & $2(1)$ & $0(1)$ \\
\hline $\mathrm{P} 2$ & $17(1)$ & $17(1)$ & $14(1)$ & $0(1)$ & $1(1)$ & $0(1)$ \\
\hline $\mathrm{C} 1$ & $21(2)$ & $12(2)$ & $19(2)$ & $2(1)$ & $4(2)$ & $-2(2)$ \\
\hline $\mathrm{C} 2$ & $22(2)$ & $20(2)$ & $19(2)$ & $1(1)$ & $1(2)$ & $2(2)$ \\
\hline $\mathrm{C} 3$ & $17(2)$ & $20(2)$ & $32(2)$ & $1(2)$ & $4(2)$ & $-2(2)$ \\
\hline $\mathrm{C} 4$ & $28(2)$ & $21(2)$ & $23(2)$ & $1(2)$ & $9(2)$ & $-1(2)$ \\
\hline $\mathrm{C} 5$ & $24(2)$ & $21(2)$ & $18(2)$ & $1(1)$ & $6(2)$ & $2(2)$ \\
\hline C6 & $21(2)$ & $13(2)$ & $21(2)$ & $-2(1)$ & $6(2)$ & $-2(1)$ \\
\hline $\mathrm{C} 7$ & $17(2)$ & $20(2)$ & $15(2)$ & $1(1)$ & $4(2)$ & $-1(2)$ \\
\hline $\mathrm{C} 8$ & $18(2)$ & $21(2)$ & $14(2)$ & $2(1)$ & $0(2)$ & $0(2)$ \\
\hline C9 & $22(2)$ & $19(2)$ & $16(2)$ & $2(1)$ & $5(2)$ & $0(2)$ \\
\hline $\mathrm{C} 10$ & $21(2)$ & $19(2)$ & $19(2)$ & $2(1)$ & $5(2)$ & $2(2)$ \\
\hline $\mathrm{C} 11$ & $18(2)$ & $22(2)$ & $12(2)$ & $1(1)$ & $3(1)$ & $1(2)$ \\
\hline $\mathrm{C} 12$ & $19(2)$ & $17(2)$ & $14(2)$ & $-1(1)$ & $2(2)$ & $-5(2)$ \\
\hline $\mathrm{C} 13$ & $15(2)$ & $18(2)$ & $18(2)$ & $-1(1)$ & $1(2)$ & $1(2)$ \\
\hline $\mathrm{C} 14$ & $19(2)$ & $16(2)$ & $21(2)$ & $1(1)$ & $1(2)$ & $-1(2)$ \\
\hline $\mathrm{C} 15$ & $20(2)$ & $21(2)$ & $16(2)$ & $-3(1)$ & $1(2)$ & $2(2)$ \\
\hline C16 & $21(2)$ & $23(2)$ & $16(2)$ & $-4(1)$ & $-1(2)$ & $-1(2)$ \\
\hline $\mathrm{C} 17$ & $19(2)$ & $18(2)$ & $21(2)$ & $-2(1)$ & $1(2)$ & $-2(2)$ \\
\hline $\mathrm{C} 18$ & $21(2)$ & $23(2)$ & $17(2)$ & $3(1)$ & $2(2)$ & $5(2)$ \\
\hline $\mathrm{C} 19$ & $21(2)$ & $17(2)$ & $11(2)$ & $3(1)$ & $-2(2)$ & $1(2)$ \\
\hline $\mathrm{C} 20$ & $19(2)$ & $23(2)$ & $16(2)$ & $0(1)$ & $2(2)$ & $-1(2)$ \\
\hline $\mathrm{C} 21$ & $21(2)$ & $18(2)$ & $16(2)$ & $4(1)$ & $0(2)$ & $5(2)$ \\
\hline $\mathrm{C} 22$ & $24(2)$ & $18(2)$ & $14(2)$ & $-2(1)$ & $1(2)$ & $1(2)$ \\
\hline $\mathrm{C} 23$ & $18(2)$ & $22(2)$ & $13(2)$ & $2(1)$ & $-2(1)$ & $0(2)$ \\
\hline $\mathrm{C} 24$ & $22(2)$ & $16(2)$ & $16(2)$ & $1(1)$ & $4(2)$ & $5(2)$ \\
\hline $\mathrm{C} 25$ & $15(2)$ & $22(2)$ & $12(2)$ & $0(1)$ & $2(1)$ & $-1(2)$ \\
\hline $\mathrm{C} 26$ & $19(2)$ & $20(2)$ & $19(2)$ & $-1(1)$ & $4(2)$ & $-4(2)$ \\
\hline
\end{tabular}




\begin{tabular}{|c|c|c|c|c|c|c|}
\hline $\mathrm{C} 27$ & $23(2)$ & $25(2)$ & $18(2)$ & $1(2)$ & $3(2)$ & $1(2)$ \\
\hline $\mathrm{C} 28$ & $23(2)$ & $23(2)$ & $19(2)$ & $4(2)$ & $4(2)$ & $1(2)$ \\
\hline $\mathrm{C} 29$ & $22(2)$ & $19(2)$ & $24(2)$ & $2(2)$ & $8(2)$ & $-1(2)$ \\
\hline $\mathrm{C} 30$ & $20(2)$ & $22(2)$ & $16(2)$ & $-1(1)$ & $5(2)$ & $-4(2)$ \\
\hline C31 & $28(2)$ & $16(2)$ & $24(2)$ & $0(2)$ & $2(2)$ & $-1(2)$ \\
\hline C32 & $38(2)$ & $31(2)$ & $36(2)$ & $6(2)$ & $-2(2)$ & $-13(2)$ \\
\hline C33 & $33(2)$ & $27(2)$ & $50(3)$ & $-17(2)$ & $-1(2)$ & $3(2)$ \\
\hline C34 & $33(2)$ & $22(2)$ & $26(2)$ & $-2(2)$ & $-3(2)$ & $-3(2)$ \\
\hline C35 & $20(2)$ & $21(2)$ & $13(2)$ & $2(1)$ & $0(2)$ & $1(2)$ \\
\hline C36 & $21(2)$ & $44(3)$ & $17(2)$ & $-1(2)$ & $-3(2)$ & $3(2)$ \\
\hline C37 & $26(2)$ & $24(2)$ & $28(2)$ & $6(2)$ & $-3(2)$ & $1(2)$ \\
\hline C38 & $20(2)$ & $29(2)$ & $26(2)$ & $5(2)$ & $2(2)$ & $-1(2)$ \\
\hline C39 & $27(2)$ & $24(2)$ & $14(2)$ & $1(1)$ & $2(2)$ & $-1(2)$ \\
\hline $\mathrm{C} 40$ & $27(2)$ & $31(2)$ & $18(2)$ & $5(2)$ & $4(2)$ & $2(2)$ \\
\hline C41 & $30(2)$ & $27(2)$ & $28(2)$ & $2(2)$ & $10(2)$ & $6(2)$ \\
\hline $\mathrm{C} 42$ & $45(3)$ & $35(2)$ & $16(2)$ & $-2(2)$ & $4(2)$ & $-3(2)$ \\
\hline $\mathrm{C} 43$ & $30(2)$ & $19(2)$ & $23(2)$ & $0(2)$ & $3(2)$ & $-5(2)$ \\
\hline $\mathrm{C} 44$ & $44(3)$ & $16(2)$ & $58(3)$ & $-4(2)$ & $20(2)$ & $-2(2)$ \\
\hline $\mathrm{C} 45$ & $48(3)$ & $27(2)$ & $31(2)$ & $8(2)$ & $6(2)$ & $-8(2)$ \\
\hline $\mathrm{C} 46$ & $36(2)$ & $28(2)$ & $29(2)$ & $-3(2)$ & $6(2)$ & $-11(2)$ \\
\hline C47 & $18(2)$ & $19(2)$ & $19(2)$ & $-2(1)$ & $1(2)$ & $-1(2)$ \\
\hline $\mathrm{C} 48$ & $27(2)$ & $25(2)$ & $38(2)$ & $-5(2)$ & $9(2)$ & $-7(2)$ \\
\hline C49 & $19(2)$ & $40(3)$ & $24(2)$ & $-4(2)$ & $-4(2)$ & $-1(2)$ \\
\hline C50 & $28(2)$ & $26(2)$ & $24(2)$ & $-1(2)$ & $7(2)$ & $-4(2)$ \\
\hline C51 & $21(2)$ & $23(2)$ & $23(2)$ & $-4(2)$ & $7(2)$ & $-2(2)$ \\
\hline C52 & $29(2)$ & $41(3)$ & $30(2)$ & $-9(2)$ & $7(2)$ & $1(2)$ \\
\hline C53 & $33(2)$ & $31(2)$ & $40(2)$ & $-9(2)$ & $17(2)$ & $0(2)$ \\
\hline C54 & $32(2)$ & $48(3)$ & $26(2)$ & $-4(2)$ & $11(2)$ & $-2(2)$ \\
\hline C55 & $42(3)$ & $28(2)$ & $18(2)$ & $-2(2)$ & $4(2)$ & $4(2)$ \\
\hline C56 & $43(5)$ & $78(7)$ & $20(4)$ & $8(4)$ & $-8(3)$ & $-21(5)$ \\
\hline C57 & $51(5)$ & $89(6)$ & $23(4)$ & $-2(4)$ & $9(3)$ & $-26(4)$ \\
\hline C58 & $149(9)$ & $65(5)$ & 11(3) & $-2(3)$ & $6(5)$ & $32(6)$ \\
\hline C55' & $42(3)$ & $28(2)$ & $18(2)$ & $-2(2)$ & $4(2)$ & $4(2)$ \\
\hline C56' & $42(7)$ & $80(9)$ & $21(6)$ & $-7(5)$ & $19(5)$ & $17(6)$ \\
\hline C57' & $51(5)$ & $89(6)$ & $23(4)$ & $-2(4)$ & $9(3)$ & $-26(4)$ \\
\hline C58' & $149(9)$ & $65(5)$ & 11(3) & $-2(3)$ & $6(5)$ & $32(6)$ \\
\hline
\end{tabular}




\begin{tabular}{lllllll} 
C59 & $34(2)$ & $21(2)$ & $26(2)$ & $1(2)$ & $9(2)$ & $3(2)$ \\
C60 & $94(4)$ & $26(2)$ & $29(2)$ & $1(2)$ & $20(3)$ & $16(2)$ \\
C61 & $34(2)$ & $27(2)$ & $61(3)$ & $0(2)$ & $21(2)$ & $1(2)$ \\
C62 & $37(2)$ & $18(2)$ & $40(2)$ & $-2(2)$ & $7(2)$ & $1(2)$ \\
C63 & $57(4)$ & $41(5)$ & $61(5)$ & $-4(6)$ & $17(4)$ & $4(4)$ \\
C64 & $43(6)$ & $43(6)$ & $65(7)$ & $15(5)$ & $11(5)$ & $8(5)$ \\
O1 & $57(4)$ & $41(5)$ & $61(5)$ & $-4(6)$ & $17(4)$ & $4(4)$ \\
C65 & $45(6)$ & $42(6)$ & $60(6)$ & $16(5)$ & $18(5)$ & $-3(5)$ \\
C66 & $57(4)$ & $41(5)$ & $61(5)$ & $-4(6)$ & $17(4)$ & $4(4)$ \\
& & & & & & \\
\hline
\end{tabular}


Table S29. Hydrogen coordinates $\left(\times 10^{4}\right)$ and isotropic displacement parameters $\left(\AA^{2} \times 10^{3}\right)$ for neijk39.

\begin{tabular}{|c|c|c|c|c|}
\hline & $\mathrm{x}$ & $\mathrm{y}$ & z & $\mathrm{U}(\mathrm{eq})$ \\
\hline $\mathrm{H} 2 \mathrm{~A}$ & 6740 & 2114 & 2338 & 25 \\
\hline $\mathrm{H} 3 \mathrm{~A}$ & 7343 & 2451 & 1707 & 28 \\
\hline $\mathrm{H} 4 \mathrm{~A}$ & 6339 & 2487 & 988 & 28 \\
\hline $\mathrm{H} 5 \mathrm{~A}$ & 4735 & 2227 & 902 & 25 \\
\hline H8A & 4199 & -83 & 2186 & 22 \\
\hline $\mathrm{H} 10 \mathrm{~A}$ & 6070 & -1960 & 2868 & 23 \\
\hline $\mathrm{H} 12 \mathrm{~A}$ & 6540 & 816 & 3072 & 20 \\
\hline H14A & 5234 & 1604 & 3439 & 23 \\
\hline H16A & 6306 & 4134 & 3883 & 25 \\
\hline H18A & 5722 & 3544 & 2549 & 25 \\
\hline $\mathrm{H} 20 \mathrm{~A}$ & 4301 & 501 & 961 & 23 \\
\hline $\mathrm{H} 22 \mathrm{~A}$ & 2233 & -1419 & 589 & 23 \\
\hline $\mathrm{H} 24 \mathrm{~A}$ & 1920 & 699 & 1418 & 22 \\
\hline $\mathrm{H} 26 \mathrm{~A}$ & 2934 & 1982 & 547 & 23 \\
\hline $\mathrm{H} 28 \mathrm{~A}$ & 1979 & 4633 & 320 & 26 \\
\hline $\mathrm{H} 30 \mathrm{~A}$ & 2869 & 3699 & 1598 & 23 \\
\hline H32A & 4575 & -2721 & 2858 & 55 \\
\hline Н32B & 3681 & -3056 & 2480 & 55 \\
\hline $\mathrm{H} 32 \mathrm{C}$ & 3641 & -2094 & 2747 & 55 \\
\hline $\mathrm{H} 33 \mathrm{~A}$ & 5263 & -2281 & 1811 & 58 \\
\hline Н33B & 4615 & -3146 & 1885 & 58 \\
\hline $\mathrm{H} 33 \mathrm{C}$ & 5532 & -2884 & 2267 & 58 \\
\hline H34A & 3790 & -1289 & 1661 & 43 \\
\hline H34B & 3177 & -1238 & 2035 & 43 \\
\hline $\mathrm{H} 34 \mathrm{C}$ & 3208 & -2188 & 1757 & 43 \\
\hline H36A & 6861 & -617 & 3925 & 43 \\
\hline H36B & 7321 & 319 & 3779 & 43 \\
\hline $\mathrm{H} 36 \mathrm{C}$ & 7982 & -507 & 4031 & 43 \\
\hline $\mathrm{H} 37 \mathrm{~A}$ & 7639 & -2149 & 3136 & 41 \\
\hline H37B & 7091 & -2156 & 3536 & 41 \\
\hline
\end{tabular}




\begin{tabular}{|c|c|c|c|c|}
\hline H37C & 8198 & -1951 & 3646 & 41 \\
\hline H38A & 8231 & -675 & 2886 & 38 \\
\hline H38B & 8800 & -460 & 3392 & 38 \\
\hline $\mathrm{H} 38 \mathrm{C}$ & 8080 & 301 & 3121 & 38 \\
\hline $\mathrm{H} 40 \mathrm{~A}$ & 6963 & 1756 & 4338 & 38 \\
\hline H40B & 6050 & 1143 & 4124 & 38 \\
\hline $\mathrm{H} 40 \mathrm{C}$ & 6273 & 1423 & 4648 & 38 \\
\hline H41A & 4675 & 2112 & 4545 & 41 \\
\hline H41B & 4431 & 1866 & 4017 & 41 \\
\hline $\mathrm{H} 41 \mathrm{C}$ & 4331 & 2922 & 4180 & 41 \\
\hline $\mathrm{H} 42 \mathrm{~A}$ & 6775 & 3434 & 4565 & 48 \\
\hline H42B & 6109 & 3066 & 4880 & 48 \\
\hline $\mathrm{H} 42 \mathrm{C}$ & 5742 & 3863 & 4511 & 48 \\
\hline $\mathrm{H} 44 \mathrm{~A}$ & 5168 & 5656 & 3163 & 57 \\
\hline H44B & 6057 & 6347 & 3265 & 57 \\
\hline $\mathrm{H} 44 \mathrm{C}$ & 5911 & 5608 & 3640 & 57 \\
\hline $\mathrm{H} 45 \mathrm{~A}$ & 5645 & 5194 & 2467 & 54 \\
\hline H45B & 6632 & 4688 & 2488 & 54 \\
\hline $\mathrm{H} 45 \mathrm{C}$ & 6599 & 5791 & 2584 & 54 \\
\hline $\mathrm{H} 46 \mathrm{~A}$ & 7530 & 4950 & 3690 & 47 \\
\hline H46B & 7688 & 5697 & 3320 & 47 \\
\hline $\mathrm{H} 46 \mathrm{C}$ & 7827 & 4600 & 3241 & 47 \\
\hline H48A & 1445 & -2036 & 1352 & 45 \\
\hline H48B & 1115 & -2180 & 817 & 45 \\
\hline $\mathrm{H} 48 \mathrm{C}$ & 348 & -2096 & 1116 & 45 \\
\hline $\mathrm{H} 49 \mathrm{~A}$ & 279 & 107 & 502 & 44 \\
\hline H49B & -373 & -767 & 559 & 44 \\
\hline $\mathrm{H} 49 \mathrm{C}$ & 477 & -903 & 313 & 44 \\
\hline H50A & 986 & -490 & 1687 & 38 \\
\hline H50B & -65 & -650 & 1397 & 38 \\
\hline H50C & 475 & 308 & 1347 & 38 \\
\hline H52A & 5218 & -425 & 729 & 50 \\
\hline H52B & 5341 & -1403 & 490 & 50 \\
\hline $\mathrm{H} 52 \mathrm{C}$ & 5071 & -1393 & 972 & 50 \\
\hline $\mathrm{H} 53 \mathrm{~A}$ & 2986 & -2097 & 158 & 50 \\
\hline H53B & 3660 & -2420 & 627 & 50 \\
\hline
\end{tabular}




\begin{tabular}{|c|c|c|c|c|}
\hline H53C & 4052 & -2391 & 176 & 50 \\
\hline H54A & 4017 & 119 & 48 & 52 \\
\hline H54B & 3140 & -542 & -164 & 52 \\
\hline $\mathrm{H} 54 \mathrm{C}$ & 4178 & -839 & -199 & 52 \\
\hline H56A & 1022 & 3162 & -691 & 74 \\
\hline H56B & 985 & 3934 & -312 & 74 \\
\hline H56C & 794 & 2853 & -221 & 74 \\
\hline H57A & 2638 & 3760 & -700 & 81 \\
\hline H57B & 3444 & 3768 & -243 & 81 \\
\hline $\mathrm{H} 57 \mathrm{C}$ & 2584 & 4491 & -306 & 81 \\
\hline H58A & 2180 & 1690 & -105 & 115 \\
\hline H58B & 3152 & 2066 & -198 & 115 \\
\hline $\mathrm{H} 58 \mathrm{C}$ & 2230 & 2029 & -601 & 115 \\
\hline $\mathrm{H} 56 \mathrm{D}$ & 3760 & 3295 & -173 & 69 \\
\hline $\mathrm{H} 56 \mathrm{E}$ & 3196 & 2649 & -576 & 69 \\
\hline $\mathrm{H} 56 \mathrm{~F}$ & 3509 & 2234 & -75 & 69 \\
\hline H57D & 1535 & 2255 & -639 & 81 \\
\hline H57E & 1009 & 2568 & -254 & 81 \\
\hline $\mathrm{H} 57 \mathrm{~F}$ & 1810 & 1779 & -149 & 81 \\
\hline H58D & 2532 & 4489 & -364 & 115 \\
\hline H58E & 1458 & 4248 & -371 & 115 \\
\hline $\mathrm{H} 58 \mathrm{~F}$ & 1929 & 3853 & -761 & 115 \\
\hline H60A & 3005 & 5281 & 1788 & 73 \\
\hline H60B & 1992 & 4852 & 1789 & 73 \\
\hline H60C & 2120 & 5963 & 1754 & 73 \\
\hline H61 A & 695 & 4930 & 1075 & 59 \\
\hline H61B & 899 & 5433 & 636 & 59 \\
\hline H61C & 867 & 6040 & 1075 & 59 \\
\hline H62A & 3325 & 5963 & 1091 & 48 \\
\hline H62B & 2461 & 6666 & 1072 & 48 \\
\hline $\mathrm{H} 62 \mathrm{C}$ & 2504 & 6036 & 641 & 48 \\
\hline H63A & 5206 & 5185 & -1005 & 79 \\
\hline H63B & 5297 & 6004 & -637 & 79 \\
\hline H63C & 4327 & 5442 & -795 & 79 \\
\hline H64A & 5073 & 4039 & -436 & 61 \\
\hline H64B & 6048 & 4604 & -276 & 61 \\
\hline
\end{tabular}




\begin{tabular}{lrrrr} 
H65A & 6084 & 4369 & 508 & 58 \\
H65B & 5139 & 3748 & 363 & 58 \\
H66A & 5262 & 4511 & 1103 & 79 \\
H66B & 4314 & 4798 & 748 & 79 \\
H66C & 5189 & 5506 & 853 & 79 \\
\hline
\end{tabular}


Table S30. Torsion angles $\left[{ }^{\circ}\right]$ for neijk39.

\begin{tabular}{|c|c|c|c|}
\hline C13-P1-C1-C2 & $40.6(3)$ & C31-C9-C10-C11 & $-179.7(3)$ \\
\hline C7-P1-C1-C2 & $-68.9(3)$ & C9-C10-C11-C12 & $1.5(5)$ \\
\hline Fe1-P1-C1-C2 & 166.7(3) & C9-C10-C11-C35 & $-177.8(3)$ \\
\hline C13-P1-C1-C6 & $-141.3(3)$ & C8-C7-C12-C11 & $-1.8(5)$ \\
\hline C7-P1-C1-C6 & $109.2(3)$ & P1-C7-C12-C11 & 179.3(3) \\
\hline Fe1-P1-C1-C6 & $-15.1(3)$ & $\mathrm{C} 10-\mathrm{C} 11-\mathrm{C} 12-\mathrm{C} 7$ & $0.1(5)$ \\
\hline C6-C1-C2-C3 & $0.9(5)$ & C35-C11-C12-C7 & $179.3(3)$ \\
\hline $\mathrm{P} 1-\mathrm{C} 1-\mathrm{C} 2-\mathrm{C} 3$ & $179.0(3)$ & C1-P1-C13-C14 & $-157.7(3)$ \\
\hline $\mathrm{C} 1-\mathrm{C} 2-\mathrm{C} 3-\mathrm{C} 4$ & $-0.8(5)$ & C7-P1-C13-C14 & $-51.6(3)$ \\
\hline $\mathrm{C} 2-\mathrm{C} 3-\mathrm{C} 4-\mathrm{C} 5$ & $0.7(5)$ & Fe1-P1-C13-C14 & $80.7(3)$ \\
\hline C3-C4-C5-C6 & $-0.8(5)$ & C1-P1-C13-C18 & $33.5(3)$ \\
\hline $\mathrm{C} 4-\mathrm{C} 5-\mathrm{C} 6-\mathrm{C} 1$ & $0.9(5)$ & C7-P1-C13-C18 & $139.6(3)$ \\
\hline C4-C5-C6-P2 & $176.9(3)$ & Fe1-P1-C13-C18 & $-88.1(3)$ \\
\hline C2-C1-C6-C5 & $-0.9(5)$ & C18-C13-C14-C15 & $-0.8(5)$ \\
\hline $\mathrm{P} 1-\mathrm{C} 1-\mathrm{C} 6-\mathrm{C} 5$ & $-179.2(2)$ & P1-C13-C14-C15 & $-169.7(3)$ \\
\hline C2-C1-C6-P2 & $-177.3(2)$ & C13-C14-C15-C16 & $-0.8(5)$ \\
\hline P1-C1-C6-P2 & $4.5(3)$ & C13-C14-C15-C39 & 178.1(3) \\
\hline C25-P2-C6-C5 & $-38.3(3)$ & C14-C15-C16-C17 & $2.1(5)$ \\
\hline C19-P2-C6-C5 & $67.6(3)$ & C39-C15-C16-C17 & $-176.8(3)$ \\
\hline Fe1-P2-C6-C5 & $-168.1(3)$ & C15-C16-C17-C18 & $-1.7(5)$ \\
\hline C25-P2-C6-C1 & $137.8(3)$ & C15-C16-C17-C43 & $179.7(3)$ \\
\hline C19-P2-C6-C1 & $-116.3(3)$ & C16-C17-C18-C13 & $0.1(5)$ \\
\hline Fe1-P2-C6-C1 & $8.1(3)$ & $\mathrm{C} 43-\mathrm{C} 17-\mathrm{C} 18-\mathrm{C} 13$ & $178.6(3)$ \\
\hline C1-P1-C7-C12 & $89.0(3)$ & C14-C13-C18-C17 & $1.1(5)$ \\
\hline C13-P1-C7-C12 & $-20.3(3)$ & P1-C13-C18-C17 & $169.9(3)$ \\
\hline Fe1-P1-C7-C12 & $-152.2(3)$ & C25-P2-C19-C20 & $92.3(3)$ \\
\hline C1-P1-C7-C8 & $-89.9(3)$ & C6-P2-C19-C20 & $-13.1(3)$ \\
\hline C13-P1-C7-C8 & $160.8(3)$ & Fe1-P2-C19-C20 & $-133.9(3)$ \\
\hline Fe1-P1-C7-C8 & $28.9(3)$ & C25-P2-C19-C24 & $-87.1(3)$ \\
\hline C12-C7-C8-C9 & $2.2(5)$ & C6-P2-C19-C24 & $167.5(2)$ \\
\hline P1-C7-C8-C9 & $-178.8(3)$ & Fe1-P2-C19-C24 & $46.6(3)$ \\
\hline C7-C8-C9-C10 & $-0.7(5)$ & C24-C19-C20-C21 & $0.8(5)$ \\
\hline C7-C8-C9-C31 & $177.8(3)$ & P2-C19-C20-C21 & $-178.6(2)$ \\
\hline C8-C9-C10-C11 & $-1.1(5)$ & C19-C20-C21-C22 & $0.6(5)$ \\
\hline
\end{tabular}




\begin{tabular}{|c|c|c|c|}
\hline C19-C20-C21-C51 & $177.9(3)$ & C12-C11-C35-C37 & $178.6(3)$ \\
\hline $\mathrm{C} 20-\mathrm{C} 21-\mathrm{C} 22-\mathrm{C} 23$ & $-1.5(5)$ & C10-C11-C35-C38 & $118.1(3)$ \\
\hline C51-C21-C22-C23 & $-178.8(3)$ & C12-C11-C35-C38 & $-61.1(4)$ \\
\hline C21-C22-C23-C24 & $0.9(5)$ & $\mathrm{C} 10-\mathrm{C} 11-\mathrm{C} 35-\mathrm{C} 36$ & $-122.8(3)$ \\
\hline $\mathrm{C} 21-\mathrm{C} 22-\mathrm{C} 23-\mathrm{C} 47$ & $179.8(3)$ & C12-C11-C35-C36 & $57.9(4)$ \\
\hline C22-C23-C24-C19 & $0.7(5)$ & C16-C15-C39-C42 & $-2.9(5)$ \\
\hline C47-C23-C24-C19 & $-178.2(3)$ & C14-C15-C39-C42 & $178.2(3)$ \\
\hline C20-C19-C24-C23 & $-1.5(5)$ & $\mathrm{C} 16-\mathrm{C} 15-\mathrm{C} 39-\mathrm{C} 40$ & $-123.6(4)$ \\
\hline $\mathrm{P} 2-\mathrm{C} 19-\mathrm{C} 24-\mathrm{C} 23$ & $178.0(3)$ & C14-C15-C39-C40 & $57.6(4)$ \\
\hline C19-P2-C25-C26 & $-25.6(3)$ & C16-C15-C39-C41 & $117.4(4)$ \\
\hline C6-P2-C25-C26 & $83.5(3)$ & C14-C15-C39-C41 & $-61.5(4)$ \\
\hline $\mathrm{Fe} 1-\mathrm{P} 2-\mathrm{C} 25-\mathrm{C} 26$ & $-155.1(2)$ & C18-C17-C43-C45 & $3.3(5)$ \\
\hline C19-P2-C25-C30 & $155.1(3)$ & C16-C17-C43-C45 & $-178.2(3)$ \\
\hline C6-P2-C25-C30 & $-95.7(3)$ & C18-C17-C43-C44 & $123.4(4)$ \\
\hline Fe1-P2-C25-C30 & $25.6(3)$ & C16-C17-C43-C44 & $-58.1(4)$ \\
\hline $\mathrm{C} 30-\mathrm{C} 25-\mathrm{C} 26-\mathrm{C} 27$ & $1.8(5)$ & C18-C17-C43-C46 & $-117.0(4)$ \\
\hline $\mathrm{P} 2-\mathrm{C} 25-\mathrm{C} 26-\mathrm{C} 27$ & $-177.5(3)$ & C16-C17-C43-C46 & $61.6(4)$ \\
\hline C25-C26-C27-C28 & $-1.8(5)$ & C24-C23-C47-C50 & $-15.2(4)$ \\
\hline $\mathrm{C} 25-\mathrm{C} 26-\mathrm{C} 27-\mathrm{C} 55$ & $-178.0(4)$ & C22-C23-C47-C50 & $165.9(3)$ \\
\hline $\mathrm{C} 25-\mathrm{C} 26-\mathrm{C} 27-\mathrm{C} 55^{\prime}$ & $173.7(5)$ & C24-C23-C47-C48 & $-135.7(3)$ \\
\hline C26-C27-C28-C29 & $0.9(5)$ & C22-C23-C47-C48 & $45.4(4)$ \\
\hline C55-C27-C28-C29 & $177.2(4)$ & C24-C23-C47-C49 & $104.8(4)$ \\
\hline C55'-C27-C28-C29 & $-174.5(5)$ & C22-C23-C47-C49 & $-74.0(4)$ \\
\hline C27-C28-C29-C30 & $0.1(5)$ & C22-C21-C51-C52 & $-142.3(3)$ \\
\hline C27-C28-C29-C59 & $-178.1(3)$ & C20-C21-C51-C52 & $40.6(4)$ \\
\hline C28-C29-C30-C25 & $-0.1(5)$ & C22-C21-C51-C54 & $97.5(4)$ \\
\hline C59-C29-C30-C25 & $178.0(3)$ & C20-C21-C51-C54 & $-79.6(4)$ \\
\hline $\mathrm{C} 26-\mathrm{C} 25-\mathrm{C} 30-\mathrm{C} 29$ & $-0.8(5)$ & C22-C21-C51-C53 & $-21.7(5)$ \\
\hline P2-C25-C30-C29 & $178.5(3)$ & C20-C21-C51-C53 & $161.2(3)$ \\
\hline C8-C9-C31-C33 & $127.5(4)$ & C28-C27-C55-C57 & $61.0(7)$ \\
\hline C10-C9-C31-C33 & $-54.0(4)$ & C26-C27-C55-C57 & $-122.9(5)$ \\
\hline C8-C9-C31-C34 & $6.2(5)$ & C28-C27-C55-C58 & $-178.0(6)$ \\
\hline C10-C9-C31-C34 & $-175.2(3)$ & C26-C27-C55-C58 & $-1.9(8)$ \\
\hline C8-C9-C31-C32 & $-112.4(4)$ & C28-C27-C55-C56 & $-58.3(7)$ \\
\hline $\mathrm{C} 10-\mathrm{C} 9-\mathrm{C} 31-\mathrm{C} 32$ & $66.2(4)$ & C26-C27-C55-C56 & $117.8(5)$ \\
\hline C10-C11-C35-C37 & $-2.2(5)$ & C28-C27-C55'-C56' & $122.6(7)$ \\
\hline
\end{tabular}




$\begin{array}{lclc}\text { C26-C27-C55'-C56' } & -52.7(9) & \text { C30-C29-C59-C60 } & 2.3(5) \\ \text { C28-C27-C55'-C58' } & 1.0(9) & \text { C28-C29-C59-C60 } & -179.7(4) \\ \text { C26-C27-C55'-C58' } & -174.3(7) & \text { C30-C29-C59-C61 } & -118.1(4) \\ \text { C28-C27-C55'-C57' } & -117.6(7) & \text { C28-C29-C59-C61 } & 60.0(4) \\ \text { C26-C27-C55'-C57' } & 67.2(8) & \text { C63-C64-O1-C65 } & -176.8(14) \\ \text { C30-C29-C59-C62 } & 122.1(4) & \text { C64-O1-C65-C66 } & -179.5(13) \\ \text { C28-C29-C59-C62 } & -59.8(4) & & \end{array}$

Symmetry transformations used to generate equivalent atoms:

$\# 1-\mathrm{x}+1 / 2, \mathrm{y},-\mathrm{z}+1 / 2$ 